\title{
Galaxy Evolution Insights from Spectral Modeling of Large Data Sets from the Sloan Digital Sky Survey
}

\author{
by
}

\author{
Erik Andrew Hoversten
}

\begin{abstract}
A dissertation submitted to The Johns Hopkins University in conformity with the requirements for the degree of Doctor of Philosophy.

Baltimore, Maryland

October, 2007
\end{abstract}

(C) Erik Andrew Hoversten 2007

All rights reserved 


\section{Abstract}

This thesis centers on the use of spectral modeling techniques on data from the Sloan Digital Sky Survey (SDSS) to gain new insights into current questions in galaxy evolution. The SDSS provides a large, uniform, high quality data set which can be exploited in a number of ways. One avenue pursued here is to use the large sample size to measure precisely the mean properties of galaxies of increasingly narrow parameter ranges. The other route taken is to look for rare objects which open up for exploration new areas in galaxy parameter space.

The crux of this thesis is revisiting the classical Kennicutt method for inferring the stellar initial mass function (IMF) from the integrated light properties of galaxies. A large data set ( $\sim 10^{5}$ galaxies) from the SDSS DR4 is combined with more in-depth modeling and quantitative statistical analysis to search for systematic IMF variations as a function of galaxy luminosity. Galaxy $\mathrm{H} \alpha$ equivalent widths are compared to a broadband color index to constrain the IMF. It is found that for the sample as a whole the best fitting IMF power law slope above $0.5 \mathrm{M}_{\odot}$ is $\Gamma=1.5 \pm 0.1$ with the error dominated by systematics. Galaxies brighter than around $M_{r, 0.1}=-20$ 
(including galaxies like the Milky Way which has $M_{r, 0.1} \sim-21$ ) are well fit by a universal $\Gamma \sim 1.4 \mathrm{IMF}$, similar to the classical Salpeter slope, and smooth, exponential star formation histories (SFH). Fainter galaxies prefer steeper IMFs and the quality of the fits reveal that for these galaxies a universal IMF with smooth SFHs is actually a poor assumption.

Related projects are also pursued. A targeted photometric search is conducted for strongly lensed Lyman break galaxies (LBG) similar to MS1512-cB58. The evolution of the photometric selection technique is described as are the results of spectroscopic follow-up of the best targets. The serendipitous discovery of two interesting blue compact dwarf galaxies is reported. These galaxies were identified by their extremely weak $(<150)[\mathrm{N} \mathrm{II}] \lambda 6584$ to $\mathrm{H} \alpha$ emission line ratios. Abundance analysis from emission line fluxes reveals that these galaxies have gas phase oxygen abundances $12+\log (\mathrm{O} / \mathrm{H}) \sim 7.7$ to 7.9, not remarkably low, and near infrared imaging detects an old stellar population. However the measured nitrogen to oxygen ratios $\log (\mathrm{N} / \mathrm{O})<1.7$ are anomalously low for blue compact dwarf galaxies. These objects may be useful for understanding the chemical evolution of nitrogen.

Advisors: Karl Glazebrook, Rosemary Wyse 


\section{Acknowledgements}

First off I would like to thank my parents, without whom I would not be here, for all the food, clothes, and cool Christmas presents as well as keeping me out of trouble and taking an interest in my future. I would like to thank my dad for answering all of my random questions about science, agriculture, economics, medicine, football, history, politics, geography, and whatever other odd stuff was going through my head on the drives from our house into Iowa City. I learned a lot from all of that and probably would not think the same way today otherwise. I would like to thank my mom for doing all the things that moms do and allowing me to use their house as a storage facility while in Australia. I would also like to make note of the fact that I attended public schools in the United States from kindergarten through college and like to consider myself proof that Thomas Jefferson was right about public education.

I thank my advisor K. Glazebrook for all of the help getting me through the hoops and hurdles of grad school, for sharing his astronomical wisdom with me, and for letting me follow him to the other side of the Earth. I am also in debt to R. Wyse for stepping in to be my official advisor after Glazebrook left for greener 
hemispheres. I also appreciate assistance from the terrific office staff including J. Krupsaw, C. King, B. Shriver, and C. Fliegel. I especially appreciated P. Carmen's enthusiasm for parties and N. Berry's uncanny ability to deal with the shenanigans of all the goofball offices spread across the central administration at Johns Hopkins.

Grad school just isn't grad school without the grad students. I would like to thank E. Peng and C. Tremonti for invaluable help in decrypting the mysteries of the SDSS in my early days at Hopkins. A. Pope was also of great help in understanding the nuances in the SDSS and for burrito runs to Fells Point in his smooth minivan. It was fun working with $\mathrm{K}$. Chiu and even more traveling the world on the cheap with him. I had many great conversations with L. Hebb on the graveyard shift at the Bloomberg Center. On countless occasions E. Felton used his Volkswagen to save me from eating at the same dumpy Charles Village restaurants on trips downtown and to the suburbs in addition to teaching me most of what I know about condensed matter physics. D. Clarke let me live on his couch for way too long after returning from Australia which was greatly appreciated.

Special thanks goes out to all of those involved in the IGwAD experience which was a great break from the bleak years of grad school. S. Srinivasan and M. Boyer get bonus props for being there from the time of conception. K. France made it all much cooler with his drums and his Explorer to drive us to the gigs. G. Brammer gets special notice for being from Iowa and buying a keytar. W. Song will be remembered for laughing all the time and being a great friend. All of the 
characters at Frazier's in Hampden which made it all so memorable deserve special thanks. J. Dinolt gets top billing for ruming the show. No one in the world writes funnier, or cruder, songs than U. Shake. I hope someone gives you a record deal. B. von Wasted deserves thanks for driving us home on several occasions in his "Party Tim" truck. T. Buckets never met a beat he couldn't ignore. Playing with P. C. C. may be the quintessential Baltimore experience. Even though we don't like him and we douk: it's his real name B. Colorado made it all the more interesting. Marty the manager gets a special nod for being such a huge fan and consistently coming up with new ways to kind of creep us out.

I would like to thank the faculty, staff, and grad students at the Centre for Astrophysics and Supercomputing at Swinburne University in Australia for being exceptionally friendly and helping me feel at home during my six month visit. I would also like to thank my roommates and neighbors at Swinburne, M. Blonde, M. Jackson, and J. Madlener, for letting me live vicariously through their undergraduate antics at a time when I was in dire need of cheering up.

I thank A. Lewis for forcing me out of the Bloomberg Center and being completely unable to pick me out of a police lineup. K. Fredericksen is a great friend and her inside scoop on what Baltimorons do to land in the hospital provides endless entertainment.

My thesis work was largely funded by the David and Lucille Packard Foundation and teaching assistantships from the Krieger School of Arts and Sciences at 
JHU.

This document was typeset using LTEX. Special thanks from myself, and I'm sure many other JHU Ph.D.s, are due to I. Goh for creating the Hopkins-specific. thesis IATEX macros used here and also to M. Clapp for his updates, and for not being annoyed that I spent so much time at his house with his roommate. C. Danforth also merits mention for his extremely helpful webpage for trouble shooting $\mathrm{IAT}_{\mathrm{E} X}$ theses at JHU.

Many of the plots in this thesis were created using helpful IDL routines written by D. J. Schlegel.

The bulk of this thesis utilizes data from the Sloan Digital Sky Survey. Here is the standard SDSS acknowledgement:

Funding for the creation and distribution of the SDSS Archive has been provided by the Alfred P. Sloan Foundation, the Participating Institutions, the National Aeronautics and Space Administration, the National Science Foundation, the U.S. Department of Energy, the Japanese Monbukagakusho, and the Max Planck Society. The SDSS Web site is http://www.sdss.org/.

The Participating Institutions are The University of Chicago, Fermilab, the Institute for Advanced Study, the Japan Participation Group, The Johns Hopkins University, the Max-Planck-Institute for Astronomy (MPIA), the MaxPlanck-Institute for Astrophysics (MPA), New Mexico State University, Princeton University, the United States Naval Observatory, and the University of Washington.

Thanks is also due to the Apache Point Observatory $3.5 \mathrm{~m}$ telescope observing specialists, J. Barentine, R. McMillan and G. Saurage in particular, for their knowledge and assistance with my observations.

This research has made use of the X-Rays Clusters Database (BAX) which is operated by the Laboratoire d'Astrophysique de Tarbes-Toulouse (LATT), under 
contract with the Centre National d'Etudes Spatiales (CNES) 


\section{Contents}

Abstract

Acknowledgements

List of Figures $\quad$ xi

List of Tables xiv

1 Introduction 1

1.1 A Picture of the Nature of Galaxies . . . . . . . . . . . . . 5

1.2 Stars: Building Blocks of Galaxies . . . . . . . . . . . . . . . . . . 9

1.2.1 The Physics of Star Formation . . . . . . . . . . . . . 12

1.3 Observational Data . . . . . . . . . . . . . . . . . . . . . . . 24

1.3 .1 Photometry . . . . . . . . . . . . . . . . . . 24

1.3 .2 Filter Sets . . . . . . . . . . . . . . . . . . 25

1.4 The Sloan Digital Sky Survey . . . . . . . . . . . . . . . . 27

1.5 The Universality of the Stellar Initial Mass Function . . . . . . . . . . . 29

1.5.1 Observational Evidence . . . . . . . . . . . . . . . 31

1.5.2 Theoretical Expectations . . . . . . . . . . . . . . . 49

1.6 Windows to the Pasts of Galaxies . . . . . . . . . . . . . . 56

1.7 Lyman Break Galaxies . . . . . . . . . . . . . . . . . . . . . . . . . 59

1.7.1 Gravitational Lensing . . . . . . . . . . . . . . . . . 61

1.7.2 Known Strongly Lensed LBGs . . . . . . . . . . . . . . . . . 64

1.8 Extremely Metal Poor Galaxies . . . . . . . . . . . . . . . . . 71

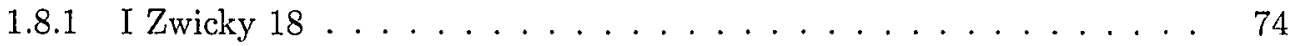

1.8.2 Properties of Other Known XMPGs . . . . . . . . . . . 76

2 The Stellar Initial Mass Function: Sample Selection and Preliminaries $\mathbf{8 3}$

2.1 Methodology . . . . . . . . . . . . . . . . . . 83

2.2 The Data . . . . . . . . . . . . . . . . . . . . 86

2.2 .1 Sample Selection . . . . . . . . . . . . . . . . . 88

2.2 .2 Corrections . . . . . . . . . . . . . . . . 96

2.2 .3 Errors . . . . . . . . . . . . . . . . . . . . . . 98

2.3 Spectral Synthesis Models . . . . . . . . . . . . . . . . . . . 99 
2.4 Statistical Techniques . . . . . . . . . . . . . . . . 108

3 Evidence for a Non-Universal Stellar Initial Mass Function 121

3.1 Monte Carlo Results . . . . . . . . . . . . . . . . . . . . . . . . . 121

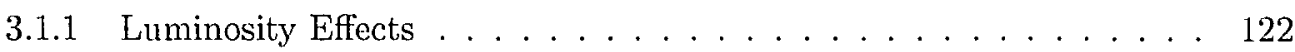

3.1 .2 Sources of Bias . . . . . . . . . . . . . . . . . . . . . 124

3.1 .3 Star Formation History . . . . . . . . . . . . . . . . . 136

$3.2 \mathrm{H} \delta_{\mathrm{A}}$ Absorption . . . . . . . . . . . . . . . . . . . . . . 160

3.2.1 Three Parameter Monte Carlo Analysis . . . . . . . . . . . . . 165

3.3 Conclusions . . . . . . . . . . . . . . . . . . 168

4 Photometric Algorithms for Selecting Lensed Lyman Break Galaxies 181

4.1 LBGs in Color Space . . . . . . . . . . . . . . . . . . . . . . . . . . . . 182

4.2 Ins and Outs of the SDSS Data . . . . . . . . . . . . . . . . 186

4.2 .1 SDSS Magnitude Systems . . . . . . . . . . . . . . . 186

4.2 .2 SDSS Data Flags . . . . . . . . . . . . . . . . . . . . . . 189

4.3. L.BG Target Selection Algorithms . . . . . . . . . . . . . . . . . . . . 191

4.3.1 First Generation Target Selection . . . . . . . . . . . . . . . . . 191

4.3.2 Second Generation Targeting Algorithm . . . . . . . . . . . . . . 197

4.3 .3 Current LBG Selection . . . . . . . . . . . . . . 210

5 Spectroscopic Study of Lensed Lyman Break Galaxy Candidates $\quad 242$

5.1 Spectroscopic Results . . . . . . . . . . . . . . . . . . . . 242

5.1 .1 First Generation Algrithm . . . . . . . . . . . . . . . . 242

5.1 .2 Second Generation Algorithm . . . . . . . . . . . . . . . . 246

5.1 .3 Current Algorithm . . . . . . . . . . . . . . . . . . . 247

5.2 Mining Known LBGs from the SDSS . . . . . . . . . . . . . . . . . . . 249

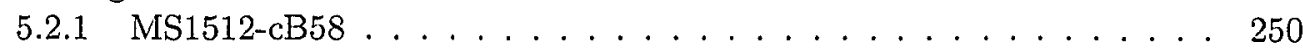

5.2 .2 The Bullet Cluster . . . . . . . . . . . . . . . . . . . . 251

5.2 .3 The Cosmic Eye . . . . . . . . . . . . . . . . . . 252

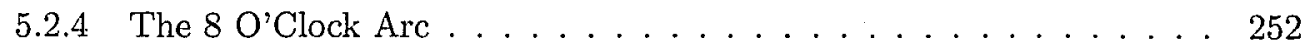

5.3 Other LBG Searches . . . . . . . . . . . . . . . . . . . . . . 254

$5.3 .1 u$-band Selected Galaxy Survey . . . . . . . . . . . . . . . 254

5.3 .2 SDSS J113658.36+024220.1 . . . . . . . . . . . . . . . . . . 261

5.3.3 SDSS Southern Stripe Image Co-Add . . . . . . . . . . . . 265

6 Extremely Metal Poor Galaxies $\quad 280$

6.1 Target Selection . . . . . . . . . . . . . . . . . . . . . 283

6.2 Metallicity Measurement Techniques . . . . . . . . . . . . . . . . . 287

6.2.1 Five-Level Approxinnation Methods . . . . . . . . . . . . . . 289

6.2 .2 Line Ratio Methods . . . . . . . . . . . . . . . . . . . . . 296

6.3 Near Infrared Photometry . . . . . . . . . . . . . . . . . . . . . 299

6.4 Discussion . . . . . . . . . . . . . . . . . . . . 304 
$\begin{array}{lll}7 & \text { Conclusions } & \mathbf{3 1 7}\end{array}$

7.1 Future Work . . . . . . . . . . . . . . . . . . . . . 324

$\begin{array}{ll}\text { A Spectroscopic Observations of LBG Candidates } & \mathbf{3 2 8}\end{array}$

$\begin{array}{ll}\text { Bibliography } & 335\end{array}$

$\begin{array}{ll}\text { Vita } & 345\end{array}$ 


\section{List of Figures}

1.1 Empirical spectra for selected main sequence stars . . . . . . . . . . . 77

1.2 Lognormal Distributions . . . . . . . . . . . . . . . . . . . . . . . 78

1.3 Selected stellar IMFs . . . . . . . . . . . . . . . . . . . . . . . . 79

1.4 SDSS J1004+4112: Gravitationally Lensed Quasar . . . . . . . . . . . . . 80

1.5 HST image of the extremely low metallicity blue compact dwarf galaxy I $\mathrm{Zw}$

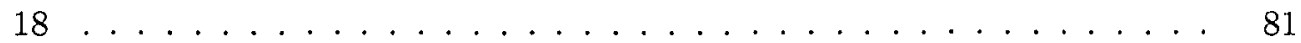

1.6 SDSS spectrum of $\mathrm{I} \mathrm{Zw} 18$, one of the most metal poor galaxies known . . . 82

2.1 Distribution of all 130,602 program galaxies in the $(g-r)_{0.1}$ vs. $\log (\mathrm{H} \alpha \mathrm{EW})$ plane . . . . . . . . . . . . . . . . . . . . . 112

2.2 Star formation histories for which models were calculated . . . . . . . 113

2.3 Model spectra with varying ages . . . . . . . . . . . . . . . . . . . . . 114

2.4 Model spectra with varying SFHs . . . . . . . . . . . . . . . . . . 115

2.5 Model spectra with varying IMFs . . . . . . . . . . . . . . . . 116

2.6 Model spectra with varying metallicities . . . . . . . . . . . . . . 117

2.7 Examples of the degeneracy of IMF parameters . . . . . . . . . . . . 118

2.8 Effects of model parameters in the $(g-r)_{0.1}$ vs. $\log (\mathrm{H} \alpha \mathrm{EW})$ plane . . . . 119

2.9 Model values in the $(g-r)_{0.1}$ vs. $\log (\mathrm{H} \alpha \mathrm{EW})$ plane for $\Gamma=1.00, \Gamma=1.35$,

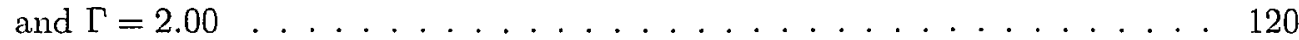

3.1 Value of $\chi^{2}$ as a function of $\Gamma$ for the entire sample . . . . . . . . . . 139

3.2 MC simulation results for the full sample binned by $\mathrm{M}_{r, 0.1} \ldots \ldots \ldots \ldots$

3.3 MC simulation results for the full sample binned by $\mathrm{M}_{z, 0.1} \ldots \ldots \ldots \ldots 141$

3.4 Definition of volume limited magnitude bins . . . . . . . . . . . . . . . 142

3.5 MC simulation results as a function of $\mathrm{M}_{r, 0.1}$ where the magnitude bins are volume limited . . . . . . . . . . . . . . . . . . . . 143

3.6 Values of fitted parameters as a function of both luminosity and redshift . . 144

3.7 Values of fitted parameters for 2-D bins cut on luminosity and aperture fraction 145

3.8 Values of fitted parameters for 2-D bins cut on luminosity and emission line extinction . . . . . . . . . . . . . . . . . . 146

3.9 Best fitting $\Gamma$ values as a function of aperture fraction and measured emission line extinction for six volume limited luminosity bins . . . . . . . . . . . 147 
3.10 MC simulation results binned by $\mathrm{M}_{r, 0.1}$ for the star forming and class low $\mathrm{S} / \mathrm{N}$ star forming classes . . . . . . . . . . . . . . . . . . . 148

$3.11 \mathrm{H} \alpha$ flux as a function of $\Gamma$ and metallicity at constant SFR . . . . . . . . . 149

3.12 Value of the $f$ ratio for the whole sample assuming $\Gamma=1.35 \ldots \ldots \ldots 150$

3.13 Best fitting $f$ ratios for volume limited magnitude bins . . . . . . . . . . . 151

3.14 Distributions in $(g-r)_{0.1}-\log (\mathrm{H} \alpha \mathrm{EW})$ space of $\mathrm{M}_{r, 0.1}=-17$ and -23

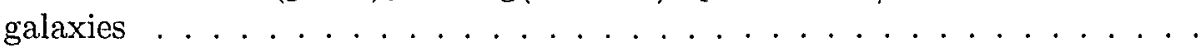

3.15 Color and $\mathrm{H} \alpha \mathrm{EW}$ values as a function of age for a model galaxy experiencing

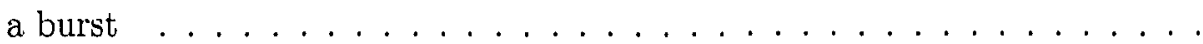

3.16 Best fitting IMF slope as a function of age for the model galaxy experiencing a burst of star formation . . . . . . . . . . . . . . . . . 154

3.17 Distributions of best fitting parameters for the synthetic $M_{r, 0.1}=-17$ data for models with $Z=0.01$ and single burst SFHs . . . . . . . . . . . . 175

3.18 Distributions of best fitting parameters for the synthetic $M_{r, 0.1}=-17$ data for models with $Z=0.01$ and multiple burst SFHs $\ldots \ldots \ldots 176$

3.19 Distribution of $\mathrm{H} \delta_{\mathrm{A}}$ values for the $\mathrm{M}_{r, 0.1}=-17$ and $\mathrm{M}_{r, 0.1}=-23$ volume limited bins . . . . . . . . . . . . . . . . . . . 177

3.20 Model values of the $\mathrm{H} \delta_{\mathrm{A}}$ index as a function of age for four IMF models . . 178

3.21 Model $\mathrm{H} \delta_{\mathrm{A}}$ values as a function of age for two multiple burst models . . . . 179

3.22 Three parameter MC simulation results for the full sample binned by $M_{r, 0.1} \quad 180$

4.1 Composite Spectra of $z \sim 3$ LBGs . . . . . . . . . . . . . . . . . . 229

4.2 Rest-frame Ultraviolet Spectrum of cB58 . . . . . . . . . . . . . . . 230

4.3 Model LBG Spectrum . . . . . . . . . . . . . . . . . . . . . . . . 231

4.4 LBG Track in $u-g$ vs. $g-r$ Color Space . . . . . . . . . . . . 232

4.5 LBG Track in $g-r$ vs. $r-i$ Color Space . . . . . . . . . . . . . 233

4.6 LBG Track in $r-i$ vs. $i-z$ Color Space . . . . . . . . . . . . . . . . 234

4.7 Typical Scatter in SDSS Photometry Data in Color Space . . . . . . . . . 235

4.8 First generation LBG color cuts . . . . . . . . . . . . . . . . 236

4.9 Hyperz results for cB58 with and without flux correction modification . . . 237

4.10 Positions of known galaxy clusters . . . . . . . . . . . . . 238

4.11 SDSS Images of Lensed LBGs . . . . . . . . . . . . . . . . . . . . . 239

4.12 Photometric redshifts of targeted galaxies . . . . . . . . . . . . . 240

4.13 Current LBG color cut . . . . . . . . . . . . . . . . . . . . . . . . 241

5.1 High redshfit quasar spectra observed in July $2001 \ldots \ldots$. . . . . . . . 270

5.2 Subaru spectra of candidate lensed LBGs . . . . . . . . . . . . . . 271

5.3 Failure of the SDSS imaging star/galaxy classification . . . . . . . . . . 272

5.4 DIS III spectrum of candidate lensed LBG SDSS J142617.23+390145.8 . . . 273

5.5 DIS III spectrum of candidate lensed LBG SDSS J154718.85+205619.5 . . . 274

5.6 DIS III spectrum of candidate lensed LBG SDSS J100051.00+232610.0 . . 275

5.7 Photometric redshifts for the 8 O'Clock Arc and parts thereof . . . . . . . . 276

5.8 Processing of Spectra for Cross-Correlation . . . . . . . . . . . . . 277

5.9 Sample Cross-Correlation Results . . . . . . . . . . . . . . . . . 278

5.10 Spectrum of the Lyman $\alpha$-only quasar SDSS J113658.36+024220.1 _ . . . 279 
6.1 Spectrum of the low metallicity galaxy SDSS J094725.23-000509.3 _ . . . 308

6.2 Close up of the spectrum of the low metallicity galaxy SDSS J094725.23-

000509.3 around the $\mathrm{H} \alpha$ emission line . . . . . . . . . . . . . . . . . . 309

6.3 SDSS images of XMPG candidates . . . . . . . . . . . . . . . . . 310

6.4 Spectrum of the low metallicity galaxy SDSS J154311.30+011235.4 . . . . . 311

6.5 Close up of the spectrum of the low metallicity galaxy SDSS J154311.30+011235.4 around the $\mathrm{H} \alpha$ emission line . . . . . . . . . . . . . . . . . . . 312

6.6 Spectrum of the galaxy SDSS J023958.57-003149.1 . . . . . . . . . . 313

6.7 Close up of the spectrum of the low metallicity galaxy SDSS J023958.57003149.1 around the $\mathrm{H} \alpha$ emission line . . . . . . . . . . . . . . . . 314

6.8 BPT diagram of low metallicity galaxy candidates . . . . . . . . . . 315

6.9 Histograms of candidate galaxy metallicities as measured from the $\mathrm{N} 12_{\mathrm{n}} / \mathrm{H} \alpha$ and $\mathrm{O} 3 \mathrm{~N} 2$ ratios . . . . . . . . . . . . . . . . 316

$6.10 H$ and $K_{S}$ images of the low metallicity galaxy SDSS J094725.23-000509.3 . 316 


\section{List of Tables}

1.1 Characteristics of Main Sequence Stars by Spectral Type . . . . . . . . . 10

1.2 Properties of Selected Astronomical Filters . . . . . . . . . . . . 26

1.3 Known strongly lensed LBGs. . . . . . . . . . . . . . . . . . . . . . . . 64

3.1 Luminosity Bin Details . . . . . . . . . . . . . . . . . . . . . . . 126

3.2 Multi-burst SFH Models . . . . . . . . . . . . . . . . . . . 158

4.1 SDSS Softening Parameter and Limiting Magnitudes . . . . . . . . . . . . 188

5.1 SDSS Photometric Data for Known Strongly Lensed LBGs . . . . . . . . 250

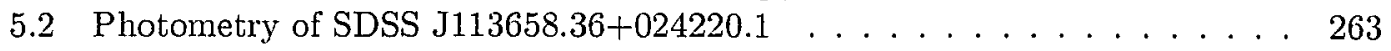

6.1 Measured oxygen abundances of candidate XMPGs . . . . . . . . . . . . . 297

6.2 Measured parameters of candidate XMPGs . . . . . . . . . . . . . . 304

A.1 Spectroscopically Identified LBG Candidates . . . . . . . . . . . . . 329

A.1 Spectroscopically Identified LBG Candidates . . . . . . . . . . . . . . 330

A.1 Spectroscopically Identified LBG Candidates . . . . . . . . . . . . . . . . 331

A.1 Spectroscopically Identified LBG Candidates . . . . . . . . . . . . . . . . 332

A.1 Spectroscopically Identified LBG Candidates . . . . . . . . . . . . . . . 333

A.1 Spectroscopically Identified LBG Candidates . . . . . . . . . . . . . . . 334 


\section{Chapter 1}

\section{Introduction}

With the increasing urbanization of life in the industrialized world a growing fraction of the populace has never experienced the full splendor of the night sky. From the hearts of the largest cities only the planets and the brightest stars can be seen. However a trip to the countryside on a clear, moonless night reveals what has been known by all people born in the millennia of human civilization before the omnipresence of street lights. A diffuse band of light stretches from one edge of the sky to the other.

This mysterious band of light has inspired cultures across the globe. The Chinese refer to it as the Silver River, a body of water which separated two lovers represented by the stars Altair and Vega. Australian aborigines view it as the smoke from the sacrificial offerings of their ancestors. In many cultures it is viewed as a pathway. The Vikings saw it as the path to Valhalla. Many other cultures, such as the Seminoles, likewise saw it as the path the dead follow into the afterlife. The Fimns and Estonians call it the Way of Birds at its arc across the sky traces the path taken by migratory birds. In Spanish it is called $E l$

Camino de Santiago or The Road to Santiago. According to this legend the belt of light is the dust made by travelers on the Way of St. James, one of the most important Catholic 
pilgrimages in medieval times. A plenary indulgence could be earned for making the trip to the Cathedral of Santiago de Compostella in the far northwest corner of Spain which is thought to be the final resting place of St. James the Great.

The legend with the largest impact in the English speaking world comes from the Ancient Greeks. They believed the band of light was the milk spilled from the breast of the goddess Hera when she awoke to find she was nursing an unknown baby. Her husband Zeus had put his illegitimate son Heracles, born by a mortal woman, in a position to drink Hera's divine milk so that he would become immortal. The Greeks called the band of light

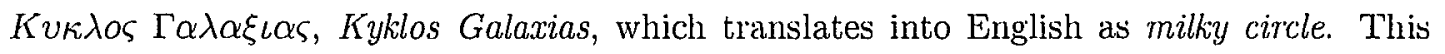
was translated into Latin as Via Lactea and eventually to English as the Milky Way. The first known use of the name Milky Way in English comes from Geoffrey Chaucer in around 1380 in his poem The House of Fame:

Se yonder, loo, the Galaxie Which men clepeth the Milky Wey, For hit ys whit

In addition to its inspirational power in the spiritual realms the Milky Way has provoked a great deal of scientific thought and discovery. The ancient Greek philosopher Democritus (460 BC - $370 \mathrm{BC}$ ) was the first person to correctly surmise that the Milky Way was in fact due to the light from many distant stars (Lynn 1901). Galileo was the first to use a telescope to prove this conjecture correct, a fact he published in Sidenus Nuncius in 1610. Immanucl Kant, known mostly for his contributions to philosophy, also made important contributions to the study of the galaxy. He described how the disk structure of the Solar System naturally arises from the combination of the Sun's gravity, which holds the Solar System together, and the rotation which keeps it from collapsing. He postulated 
that a similar situation could be at play in the Milky Way which would agree with the observed structure of a band of light across the sky. He attributed the lack of evidence for this rotation to the large size of the Milky Way which renders it imperceptible. He went further to suggest that what were then called nebulae, faint, fuzzy patches of light, could possibly be complete island universes which were themselves similar to the Milky Way but at great distances.

In the 18th century more powerful telescopes allowed Charles Messier and William Herschel, his sister Caroline Herschel to catalog many more nebulae. William Parsons used the world's largest telescope at the time to observe spiral structure in many of the nebulae which supported Kant's earlier suggestion of rotation in those objects. Dreyer (1888) combined the Herschel's nebulae along with other objects which had been discovered since into the New General Catalogue, known as the NGC.

The basic model of the Milky Way became complete in the early twentieth century. The Great Debate took place on April 26, 1920. Harlow Shapley thought that the Milky Way was the entire universe and that all of the observed nebulae, such as those in the NGC, were contained within it. One reason was that the calculated distance to the Andromeda Nebula must be 100 million light years if it was similar in size and not part of the Milky Way. Another issue was that a notable astronomer of the time Adriaan van Maanen claimed to have observed the Andromeda Nebula rotating. If the Andromeda Nebula had a size similar to the Millsy Way it would require that stars on the edge move faster than the specd of light. Heber Curtis took the position that the nebula were island universes external to the Milky Way. His reasoning was that more novae were observed in Andromeda than than in 
the rest of the Milky Way combined. Such a strong concentration of novae in one area of the Milky Way would be hard to explain. In addition, given the observed angular sizes of spiral nebulae, if they were of similar physical size they must differ in distance by a factor of 1000. The observed Doppler shifts of nebulae were found to be much higher than that of the stellar population in the Milky Way and the spectra of nebulae resembled what you would get from comparing all of the stars in the Milky Way.

In the end, van Maanen's observation of the rotation of Andromeda was incorrect and the true nature of spiral nebulae as island universes was revealed. Given the allencompassing connotation of the word universe the term galaxy came to be used to describe a whole class of objects and not just limited to the Milky Way. Within years Edwin Hubble had developed his tuning fork galaxy classification system which divided galaxies by morphology into ellipticals and spirals, with the spirals subdivided into those which possess a bar structure in the center and those which do not.

With each question regarding galaxies that is answered many new ones appear. Over the past century it has become possible through technological advances in instrumentation and a growing knowledge base to continue unraveling the many mysteries of galaxies. In addition to working towards a deeper understanding of how galaxies are today much effort is put into understanding how galaxies formed and evolved into what they are today.

This thesis continues this long tradition of the exploration of island universes in ways some of the pionecrs of the ficld could never have imagined. Data from the Sloan Digital Sky Survey, which contains millions of galaxies, is used to address questions in galaxy evolution. The remainder of this chapter provides background information for the 
projects ahead. At the core of this thesis is a study of the stellar initial mass function (IMF) in galaxies in Chapters 2 and 3. The IMF dictates the fraction of stars born at a given mass and has a large effect on the chemical evolution of galaxies, galactic winds and the interpretation of their observed parameters, as well as it provides clues to the physics of star formation and, from a cosmological perspective, the reionization of the Universe. Other projects related to galaxy evolution are also included. Chapters 4 and 5 detail a targeted search for Lyman Break Galaxies which are strongly lensed by intervening clusters. These objects are the progenitors of present day galaxies and understanding them is key to obtaining a full picture of galaxy evolution. Chapter 6 details observations of candidate extremely metal poor galaxies. As metals are not formed in the Big Bang they accumulate only through successive generations of star formation and death. Extremely metal poor conditions provide insight into the conditions of the early universe and thus the evolution of galaxies. Finally conclusions are presented in Chapter 7.

\subsection{A Picture of the Nature of Galaxies}

According to the current conventional cosmological wisdom at present the Universe is predominantly comprised of dark energy. This accounts for $74 \%$ of the Universe (Spergel, et al. 2003). Of the remainder $22 \%$ is composed of dark matter. Only $4 \%$ is baryonic matter, that which is composed of protons and neutrons. Little is known about dark energy and dark matter. Only the $4 \%$ of the Universe is made of the types of matter we are farniliar

with. Much of what is known about the Universe on large scales comes from the study of galaxies which are tracers of baryonic matter. As such knowledge of galaxies is fundamental 
to our understanding of the Universe. Galaxies are complex and interesting objects in their own right with a wide range of physical properties.

The menagerie of galaxies began to be appreciated with the Hubble tuning fork diagram, a classification system for the morphologies of galaxies. At one end are elliptical galaxies which have a smooth and structureless appearance. Ellipticals are subdivided into groups based on their apparent ellipticity. Ellipticals which appear round are given a classification of E0 while the most elongated are dubbed E7. On the other end of the scheme are spiral galaxies. These galaxies have a bright central part, which resembles an elliptical galaxy, at the center of a thin disk of spiral shaped regions of relatively increased brightness, called the spiral arms. Spirals are split into two groups depending on whether or not they have a bar shaped structure at the center. Spirals are further classified by the prominence of the central bulge and how tightly wound the spiral arms are. For instance, an Sa galaxy is a spiral galaxy with a prominent bulge and tightly wound arms and an SBd is a barred spiral with a small central bulge and loosely wound arms.

In between the ellipticals and spirals are the lenticular galaxies, denoted by $\mathrm{S} 0$ and SB0, which have smooth bright centers like an elliptical but surrounded by a structureless disk of decreasing brightness. In addition there are irregular galaxies which do not show any symmetry or spiral arms and of ton have regions of young stars. For incorrect historical reasons ellipticals are often referred to as early-type galaxies while spirals are called late-type galaxies.

The radial surface brightness profiles of galaxies can be measured. The radial 
profiles of cllipticals are observed to follow a de Vaucouleurs profile given by

$$
I(R)=I_{e} \exp \left\{-7.67\left[\left(R / R_{e}\right)^{1 / 4}-1\right]\right\}
$$

where $R_{e}$ is the effective radius. The effective radius is defined as the radius which contains half of the total luminosity of the galaxy. $I_{e}$ is the surface brightness at the effective radius. The central bulges of spirals are also well fit by a de Vaucouleurs profile. However the disks of spiral are fit by an exponential profile, defined by

$$
I(R)=I_{d} \exp \left(-R / R_{d}\right)
$$

where $R_{d}$ is the disk scale length. Spiral galaxies are fit by a sum of a de Vaucouleurs and exponential profile.

The galaxy luminosity function is another important concept in the study of galaxies. The galaxy luminosity function is the volume density of galaxies in space as a function of luminosity. Galaxy luminosity functions are usually fit by Schechter functions. For galaxies fainter than a characteristic luminosity called $L_{*}$, of $M_{*}$ when expressed in absolute magnitudes, the density of galaxies is roughly constant. However for galaxies brighter than $L_{*}$ the space density of galaxies drops exponentially as a function of luminosity. The value of $L_{*}$ is roughly 10 billion solar luminosities in the $R$ band. The galaxy luminosity function provides clues into the formation and evolution of galaxies.

The most studied spiral galaxy is our own Milky Way. When discussing the Milky Way as opposed to another galaxy or galaxies in general the terms galaxy, galactic, and other derivatives are capitalized. The Milky Way is divided into several components. At the center is the Galactic bulge, which may or may not have a bar structure. The bulge has a 
radius of about $1 \mathrm{kpc}$. The bulge stars have the highest velocity dispersion of any Galactic. component. There is a thin disk with a scale height of $325 \mathrm{pc}$ and a thick disk of scale height $1.4 \mathrm{kpc}$. The thin disk has roughly solar metallicity while the thick disk has lower metallicities. The thin disk is an order of magnitude more massive than the thick disk. The stars in the thick disk have a larger velocity dispersion than the thin disk which is why it is thicker. Most of the baryonic mass of the galaxy is in the thin disk. The stellar halo is comprised of globular clusters and field stars with large velocity components perpendicular to the plane of the Galaxy. The stellar halo is home to the most metal poor stars in the galaxy and is thus thought to be older. Lastly most of the mass of the galaxy is contained in the dark matter halo which is thought to be roughly spherical with a radius of at least $100 \mathrm{kpc}$. The Sun is located about $8 \mathrm{kpc}$ from the Galactic center.

In the immediate vicinity there are many dwarf galaxies which orbit the Milky Way. The most prominent of these are the Large and Small Magellanic Clouds which can easily be observed with the naked eye from a dark location in the southern hemisphere. The Milky Way is one of the largest members of the Local Group, an association of galaxies within 1 Mpc. The Milky Way is on a collision course with the Andromeda, a spiral galaxy similar to the Milky Way and fellow member of the Local Group. It is thought that the two galaxies will collide in six billion years. Galaxies frequently have interactions. There is evidence that the Milky Way consumes nearby dwarf galaxies and galaxy mergers are thought to play a crucial role in the mass assembly of galaxies.

Galaxies are not uniformly distributed in space. Multiple galaxies make up groups, groups make up clusters, and clusters make up superclusters. The Local Group is part of 
the Virgo Supercluster. Surveys of clustering reveal large voids which are free of galaxies. Galaxies cluster on scales over several orders of magnitudes.

Many galaxies, including possibly the Milky Way, contain supermassive black holes, with masses of several hundred solar masses, at their centers. During some periods over the course of a galaxies lifetime these black holes can form disks of gas, dust and stars which accrete onto the central black hole. The accretion process is far more efficient at releasing energy than the nuclear reactions powering stars. As such these accretion disks can have staggering luminosities. Galaxies which are presently experiencing such activity are called active galactic nuclei. Quasars are a class of AGN. The name comes from their quasi-stellar appearance. Although they may be otherwise normal spiral or elliptical galaxies the light from the accretion disk outshines the rest of the galaxy combined. This radiation originates in a small region around the black hole which cannot be resolved at cosmological distances giving them a stellar appearance.

\subsection{Stars: Building Blocks of Galaxies}

To understand galaxies it is necessary to have a strong understanding of stars. The appearance of galaxies in the optical is determined by three things: the stellar population, dust, and sometimes light from the accretion of material onto a central massive black hole.

The Vogt-Russel theorem states that the mass and composition of a star uniquely determine its radius, luminosity, internal structure and subsequent evolution. In practice angular momentum and magnetic fields also influence stellar interiors. However this state-

ment works well for the majority of stars. Stars form out of the gravitational collapse of 
Table 1.1. Characteristics of Main Sequence Stars by Spectral Type

\begin{tabular}{cccccccc}
\hline \hline Type & $T_{\text {eff }}(K)$ & $L / L_{\odot}$ & $R / R_{\odot}$ & $M / M_{\odot}$ & $M_{V}$ & $B-V$ & $t(M y r)$ \\
\hline & & & & & & & \\
O5 & 44,500 & 790,000 & 15 & 60 & -5.7 & -0.33 & 3 \\
B5 & 15,400 & 830 & 4.1 & 5.9 & -1.2 & -0.17 & 50 \\
A5 & 8,200 & 14 & 1.9 & 2.0 & +1.9 & +0.15 & 1,300 \\
F5 & 6,440 & 2.9 & 1.4 & 1.4 & +3.6 & +0.44 & 3,600 \\
G2 & 5,770 & 1.0 & 1.0 & 1.0 & +4.83 & +0.68 & 10,000 \\
K5 & 4,350 & 0.15 & 0.68 & 0.67 & +7.4 & +1.15 & 33,000 \\
M5 & 3,240 & 0.011 & 0.33 & 0.21 & +12.3 & +1.64 & $1,000,000$ \\
\hline
\end{tabular}

molecular clouds, which is described in more detail in the next section. The initial composition of stars is usually around $70 \%$ hydrogen, $1-3 \%$ metals, with the remainder being helium. Hydrogen burning will ignite in condensed objects with masses larger than $\sim 0.08 M_{\odot}$ and become main sequence stars. General properties of main sequence stars are shown in Table 1.1.

Main sequence stars follow a tight relationship between surface temperature and luminosity. The spectra of stars are to first order black body spectra with effective temperatures equal to the surface temperature. Superposed over the black body spectra are photospheric absorption lines. These lines become more prominent with increasing metal content in the star, and there are more absorption lines in the blue part of the spectra. As such low metallicity stars which have weaker lines tend to be bluer than stars with stronger absorption lines from high metal content. Stars are classified into spectral types which are strongly correlated with their surface temperatures. The progression of types is, for 
historical reasons, O, B, A, F, G, K, M, L, and T from hottest to coolest. Figure 1.1 shows spectra of $\mathrm{O}, \mathrm{B}, \mathrm{A}, \mathrm{F}, \mathrm{G}, \mathrm{K}$, and $\mathrm{M}$ stars on the main sequence.

Hydrogen burning in the cores of stars stops once about $10 \%$ of the stellar mass has been converted from hydrogen to helium. Although the most massive stars have over 1,000 times more fuel to burn than the smallest stars they must do so far more quickly to support themselves against gravitational collapse. The most massive stars in Table 1.1 are 300 times larger than the smallest but their luminosities are 100 million times larger. As such the most massive stars live for only a few million years where no stars with masses less than $0.8 M_{\odot}$ have yet to complete their evolution.

The ultimate fate of stars is also strongly dependent on their mass. Stars less than $8 M_{\odot}$ eventually become white dwarf stars which have ceased all internal burning of elements and collapse to the point they are supported by electron degeneracy pressure. These stars have roughly a half solar mass confined to a volume of Earth size. Stars with masses from 8 to $60 M_{\odot}$ become neutron stars which are supported by neutron degeneracy pressure. The most massive stars collapse to become black holes.

Before reaching their final fate stars brighten significantly for a period of time which is short compared to their overall lifetimes. Stars with masses less than $8 M_{\odot}$ initially transition from core to shell burning of hydrogen after leaving the main sequence, a period which is known as the subgiant branch. The hydrogen shell burning phase is known as the red giant branch where its luminusity significantly increases. At the lop of the red giant branch stars with masses less than $\sim 2 M_{\odot}$ experience a helium flash, an explosive ignition of helium in the core. Stars then move to the horizontal branch where they burn 
helium in their cores in a manner similar to their hydrogen burning on the main sequence. The asymptotic giant branch marks a phase of shell helium burning. At the end of the asymptotic branch a star will shed its remaining hydrogen, helium burning ceases and the remainder of the star cools to become a white dwarf. While this period is relatively short due to the high luminosities of the stars during this period they make a significant contribution to the luminosities of galaxies during this time, more so than during their main sequence lifetimes in the case of stars with masses less than $8 M_{\odot}$.

\subsubsection{The Physics of Star Formation}

\section{The Jeans Mass}

As stars form from the collapse of molecular clouds into protostars it is important to understand the causes of this collapse. Under what conditions will a relatively stable cloud of gas collapse upon itself? The first relevant answer came in 1902 when Sir James Jeans derived what is now known as the Jeans criterion published in his book Astronomy and Cosmogony (Jeans 1928).

An interesting historical note is that in the review of Astronomy and Cosmogony in Science, B. Gerasimovič (Gerasimovič 1928) takes issue with Jeans' assertion that the atomic number of atoms in stellar interiors is around 50, his "polemics with Eddington" and the lack of any references to the works of Linbald or Oort but makes no mention of his model for cloud collapse.

Derivation of the Jeans criterion begins with a gas cloud which is assumed to be homogeneous with infinite extent and therefore the density, $\rho$, and temperature are 
constant everywhere. From these assumptions the Jeans criterion can be derived from just, four physical equations (Kippenhahn \& Weigert (1994), Hartmann (1998)). The equation of motion of hydrodynamics (neglecting the effects of magnetic fields at this point):

$$
\frac{\partial \mathbf{v}}{\partial t}+(\mathbf{v} \cdot \nabla) \mathbf{v}=-\frac{1}{\rho} \nabla P-\nabla \Phi
$$

the continuity equation:

$$
\frac{\partial \rho}{\partial t}+\mathbf{v} \nabla \rho+\rho \nabla \cdot \mathbf{v}=0
$$

the gravitational potential is given by Poisson's equation,

$$
\nabla^{2} \Phi=4 \pi G \rho
$$

and finally the equation of state for an ideal gas:

$$
P=v_{s}^{2} \rho
$$

where $v_{s}$ is the isothermal speed of sound. Using these equations a perturbation solution is sought. The density, pressure, velocity and potential of the gas are replaced by the equilibrium values, which are independent of space and time, plus small perturbations as in equation 1.2.1. The fact that $\rho_{0}$ and $P_{0}$ are both constant imply that $\mathrm{v}_{0}=0$.

$$
\begin{gathered}
\rho=\rho_{0}+\rho_{1} \\
P=P+P_{0} \\
\Phi=\Phi_{0}+\Phi_{1} \\
\mathbf{v}=\mathbf{v}_{0}+\mathbf{v}_{1} \rightarrow \mathbf{v}_{1}
\end{gathered}
$$


At this point it is important to point out the Jeans swindle (Binney \& Tremaine 1987). An infinite gravitating homogeneous system camnot be in static equilibrium. Given constant pressure and density and zero velocity equation 1.3 becomes $\nabla \Phi_{0}=0$. Therefore $\nabla^{2} \Phi$ must also be zero. This is inconsistent with Poisson's equation (equation 1.5) unless $\rho_{0}=0$, which is obviously not the case. It is assumed that Poisson's equation is only a description of the relationship between the perturbed potential and perturbed density.

There are instances where the Jeans swindle can be justified. In a uniformly rotating homogeneous system gravitation can be balanced by rotation. In our case we assume the wavelength of the perturbations in a molecular cloud is small comparcd to the size of equilibrium pressure and density gradients. For small-scale instabilities the Jeans swindle can be justified.

We can now substitute the perturbations of equation 1.2.1 into equations $1.3,1.4$, and 1.5. Assuming the perturbations are isothermal and ignoring second order terms the relationship between perturbed quantities can be found.

$$
\begin{gathered}
\frac{\partial \mathbf{v}_{1}}{\partial t}=-\nabla\left(\Phi_{1}+\frac{v_{s}^{2} \rho_{1}}{\rho_{0}}\right) \\
\frac{\partial \rho_{1}}{\partial t}+\rho_{0} \nabla \cdot \mathbf{v}_{1}=0 \\
\nabla^{2} \Phi_{1}=4 \pi G \rho_{1}
\end{gathered}
$$

Equations 1.11, 1.12 and 1.13 form a system of linear homogeneous differential equations. This warrants the assumption that the solutions will be proportional to $\exp [\mathrm{i}(k x+\omega t)]$. This leads to the dispersion relation:

$$
\omega^{2}=k^{2} v_{s}^{2}-4 \pi G \rho_{0}
$$


For large wave numbers $\omega^{2}$ will be positive and $\omega$ will be real. This leads to periodic perturbations which will not result in the collapse of the cloud. However, for small values of the wave number $\omega^{2}$ will be negative and $\omega$ will be imaginary. This allows the existence of perturbations that grow exponentially in time which leads to instability in the cloud. The turning point between periodic and runaway perturbations happens at a characteristic wave number

$$
k_{J}^{2}=\frac{4 \pi G \rho_{0}}{v_{s}^{2}}
$$

This corresponds to a characteristic wavelength

$$
\lambda_{J}=\frac{2 \pi}{k_{J}}=v_{s} \sqrt{\frac{\pi}{G \rho_{0}}}
$$

which is the Jeans criterion and is also know as the Jeans length. For wavelengths such that $\lambda>\lambda_{J}$ the could is unstable. The Jeans length in turn leads to a Jeans mass

$$
M_{J}=\lambda_{J}^{3} \rho_{0}=\frac{v_{s}^{3}}{\sqrt{\rho_{0}}}\left(\frac{\pi}{G}\right)^{3 / 2}
$$

above which a homogeneous cloud with density $\rho_{0}$ and sound speed $v_{s}$ is unstable. Inserting the sound speed for an ideal gas, $v_{s}^{2}=\mathrm{RT} / \mu$, gives the following:

$$
M_{J}=\left(\frac{\pi \mathrm{R}}{G \mu}\right)^{3 / 2} T^{3 / 2} \rho^{-1 / 2}
$$

Equation 1.18 is a standard expression for the Jeans mass. It reveals the temperature dependence of the Jeans mass. It can also be expressed in more physical terms by the following:

$$
M_{J}=1.2 \times 10^{5} \mathrm{M}_{\odot}\left(\frac{T}{100 \mathrm{~K}}\right)^{3 / 2}\left(\frac{\rho}{10^{-24} \mathrm{~g} \mathrm{~cm}^{-3}}\right)^{-1 / 2} \mu^{-3 / 2}
$$


The above arguments are for an infinite uniform cloud. It is important to consider more realistic geometries as well. These investigations result in Jeans masses consistent with equation 1.18 beyond geometric prefactors.

\section{Fragmentation}

Plugging typical values of $T, \rho$, and $\mu$ for interstellar gas clouds into equation 1.19 reveals that they are stable to collapse up to masses of around $10^{5} \mathrm{M}_{\odot}$. This is three orders of magnitude larger than the most massive $O$ stars observed, leaving a large discrepancy to be overcome if collapsing clouds are responsible for the formation of stars of order a solar mass.

The trick is that at such low densities the clouds are optically thin. During collapse the gravitational term $\left(4 \pi G \rho_{0}\right)$ dominates the pressure term $\left(k^{2} v_{s}^{2}\right)$ in equation 1.14, which leads to a free-fall time scale of $\tau \sim(G \rho)^{-1 / 2}$. This is larger than the thermal adjustment time so the cloud can collapse isothermally. Low \& Lynden-Bell (1976) show that, for a gas with $p \propto \rho^{\gamma}, v_{s}^{2}=\mathrm{d} p / \mathrm{d} \rho \propto \gamma \rho^{\gamma-1}$. In turn $v_{s}^{3} \rho^{-1 / 2} \propto \rho^{(3 \gamma-4) / 2}$. In cases where $\gamma<4 / 3$, such as in isothermal collapse, the Jeans mass will get smaller with increasing density. For example, when $\gamma=1, M_{J} \propto \rho^{-1 / 2}$ and when $\gamma=4 / 3$ the Jeans mass is constant.

Hoyle (1953) was the first to realize that the lowering of the Jeans mass during the isothermal collapse of a cloud, in his case dealing with the formation of galaxies, could result in the cloud itself splitting into two pieces. Those pieces could themselves split and so on resulting in hierarchical fragmentation. In his model a spherical cloud with an initial radius of $R_{0}$, density $\rho_{0}$ and temperature $T_{0}$ contracts by some factor $k^{2 / 3}$ and then splits into $k$ equal masses, each with radius $R_{0} / k$, density $\rho_{0} k^{2}$ and the same initial temperature 
$T_{0}$. All of the new pieces contract further by a factor of $k^{2 / 3}$ so that there are $k^{2}$ equal fragments with radius $R_{0} / k^{2}$, density $\rho_{0} k^{4}$ and temperature $T_{0}$. These pieces contract and split themselves and so on. The free-fall time for the cloud is proportional to $1 / \sqrt{\rho}$ so the time for each fragment to collapse and divide is $1 / k$ the length of the previous step. The ratio of the total time for all of the fragmentation steps to the time for the first step is just

$$
1+\frac{1}{k}+\frac{1}{k^{2}}+\frac{1}{k^{3}}+\cdots=\frac{k}{k-1}
$$

This shows that regardless of the value of $k$ the bulk of the time for fragmentation is in the first splitting and the rest of the fragmentation happens fairly quickly after that.

However, the reduction of the Jeans mass does not go on ad infinitum. In order to maintain a constant temperature a cloud must be able to cool. This cooling requires that the optical depth over the Jeans length must be less than about 1 so that the outside of the cloud is visible. As the density of the cloud increases it reaches a point where the optical depth becomes high enough that the thermal adjustment time cannot keep up with the free-fall time. At this point the cloud becomes adiabatic with $\gamma=5 / 3$ for a monotonic gas. When this happens the Jeans mass starts to increase with increasing cloud density ending fragmentation.

Rees (1976) noted that a cloud cannot emit radiation faster than a black body of its size. Therefore when the rate at which the cloud must dump its gravitational energy reaches some fraction of the black body radiation it will become opaque and fragmentation will stop. This argument gives a lower limit on the cloud temperature. Having found the condition under which fragmentation stops the size of the smallest fragment can also be determined. The minimum Jeans mass is an important concept in star formation as it 
gives the size of the smallest star forming fragments, and thus a lower limit on the possible masses of stars formed in the cloud. The minimum Jeans mass is very much a lower limit in that the mass of a fragment must be at least twice the Jeans mass at any time in order for the fragment to split. In addition, accretion can increase the size of stars after their initial collapse but cannot reduce their mass. Using his arguments Rees found that the minimum Jeans mass is

$$
M_{J, m i n} \simeq M_{C} \mu^{-9 / 4} f^{-1 / 2}\left(\frac{k T}{m_{p} c^{2}}\right)^{1 / 4}=0.02 \mathrm{M}_{\odot} \frac{T^{1 / 4}}{f^{1 / 2}}
$$

where $M_{C}$ is the Chandrasekhar mass and $f$ is the efficiency of the black body radiation which must be between 0 and 1 . There are a number of factors that can greatly reduce the value of $f$. A high albedo where $\kappa_{\text {scat }} \gg \kappa_{\text {abs }}$ requires that $f \lesssim \kappa_{a b s} / \kappa_{\text {scat }}$. When cooling is confined to a few narrow emission lines or caused by dust which remains cooler than the gas or if the cloud is exposed to substantial external radiation with a temperature near the cloud temperature the $f$ factor can be significantly diminished. Low \& Lynden-Bell (1976) also theoretically predicted the minimum Jeans mass. In their calculation the temperature and density dependence are incorporated into the opacity at the end of the isothermal regime $\kappa_{f}:$

$$
M_{J, \min } \simeq 0.025 \mathrm{M}_{\odot}\left(\frac{m_{\mathrm{H}}}{m}\right)^{16 / 7}\left(\frac{\kappa_{0}}{\kappa_{f}}\right)^{1 / 7}
$$

where $\kappa_{0}$ is the opacity due to free electron scattering in ionized hydrogen. The significance of this formulation is the very weak dependence of the opacity on the Jeans mass. In fact over the range of realistic opacities the minimum Jeans mass will not vary by more than a factor of 5 .

Layzer (1963) rules out the possibility of fragmentation occurring in an expanding 
cloud.

\section{Turbulence and the Fractal Nature of Hierarchical Clouds}

In his book The Fractal Geometry of Nature Mandelbrot (1983) summarizes the mathematical discipline of fractals and lists many of the instances in which arise naturally. To include the mathematical definition of a fractal would be a bit of a digression, but the basic idea is that a fractal object can be described by a non-integer dimensionality. In the case of a square the perimeter $P$ and area $A$ are related by $P=2 \sqrt{\pi} A^{1 / 2}$, and for a circle $P=4 A^{1 / 2}$. Both squares and circles have regular, smooth perimeters which are lines of dimension $D=1$. In general $P \propto A^{D / 2}$. In fact by comparing the area of the drainage basin to the length of the river it can be found that the Missouri River has $D=1.4$, a measure of how much it winds. The upper limit on the dimensionality is $D=2$ which corresponds to a plane filling curve.

As Mandelbrot mentions, the study of turbulence is one of the oldest, most difficult and most frustrating branches of physics. Unfortunately it is also one of the most important facets of molecular cloud collapse and thus star formation. He makes the observation that most studies of turbulence attack the problem in terms of analytic derivation of the fluid flow and advocates for more focus of the geometric aspects of the flow.

The idea of using self-similar fractals to study turbulence is motivated naturally from observing hierarchical eddies in water. Mandelbrot combined the analytic theory of Kolmogorov with fractal geometry and the assumption of isotropic, homogeneous turbulence. This predicts that in turbulent clouds isotherms have $D=5 / 3$ and isobars have $D=4 / 3$. This is not the full story as an important aspect of turbulence is that it is even- 
tually wiped out by dissipation. Interminttency is the fact that the strength of dissipation is not uniform in space, which greatly complicates the analysis.

Beech (1986) traced dark molecular clouds from the Palomar Observatory Sky Survey plates onto graph paper and then graphically determined their areas and perimeters. For his collection of molecular clouds he found $D=1.4$ with minimal scatter in $\log A-\log P$ space. This suggests that molecular clouds do indeed exhibit fractal geometry, and that it may be exploited for a higher understanding of turbulence in molecular clouds.

\section{The Stellar Initial Mass Function}

Put simply, the stellar initial mass function (IMF) is the birth weight distribution for stars. The IMF arises from the physical processes of molecular clouds, and perhaps stochastic processes, in an as yet poorly understood way. These processes combine to favor the formation of stars of certain masses while inhibiting or even forbidding the the creation of stars in other mass ranges. During a star formation event stars are formed in the following proportions:

$$
\mathrm{d} N=N_{0} \xi(m) \mathrm{d} m
$$

where $\mathrm{d} N$ is the number of stars formed in the mass range $m$ to $m+\mathrm{d} m$ and $\xi(m)$ is the IMF. $N_{0}$ is the normalization constant (Binney \& Merrifield 1998).

Equation 1.23 defines the IMF. Mathematically $\xi(m)$ can have any value and functional form as long as the total stellar mass formed is finite. However empirical measurements of the IMF lead to heavy constraints on its general shape and preferred parameterizations. While there are many different measurements and models of the IMF they all share the same overall shape. Extremely few massive stars are formed compared to low 
mass stars. There is also a majority opinion on the paraneterization. In the literature the relationship between the stellar mass and the relative number of stars formed in that mass bin is overwhelmingly described by piecewise power laws. This parameterization dates back to the very first IMF measurement which at the time Salpeter called an "original mass function" (Salpeter 1955). Salpeter found that the IMF was well described by a power law from 0.4 to $10 \mathrm{M}_{\odot}$. Because Salpeter's original IMF measurement is still consistent with modern measurements it is often considered standard and it is an important reference point. Unfortunately a consensus on the description of the power law slope does not exist (Kemnicutt 1998a). There are two frequently used descriptions. In equation 1.24 mass and numbers are measured in linear bins. In this formulation a Salpeter IMF has a slope $\gamma=2.35$, where $a$ is a normalization constant.

$$
\begin{gathered}
\frac{\mathrm{d} N}{\mathrm{~d} m}=a m^{-\gamma} \\
\frac{\mathrm{d}(\log N)}{\mathrm{d}(\log m)}=A m^{-\Gamma}
\end{gathered}
$$

In equation 1.25 mass and number are measured in logarithmic bins, where the Salpeter slope $\Gamma=1.35$ and $A$ is a normalization constant.

Equation 1.25 is the formulation that will be used throughout the rest of the paper. It is historically preferable as Salpeter used logarithmic bins for his original mass function and the $\Gamma$ formulation seems to be more popular today, especially in measurements of the stellar populations of galaxies outside our own. The value of $\Gamma$ can be cxpressed in terms of the IMF $\xi(m)$ as shown in equation 1.26 (Phelps \& Janes 1993).

$$
\Gamma(m)=-\left.\frac{\partial \log \xi(\log m)}{\partial \log m}\right|_{m}
$$


In addition to the slope power law parameterizations have another important input. Because power laws never reach zero the IMF is often truncated-only defined between minimum and maximum stellar masses. There are physical motivations for this truncation. Protostars with $\mathrm{M} \lesssim 0.08 \mathrm{M}_{\odot}$ lack the self gravity to ignite hydrogen burning in their cores and fail to reach the main sequence. While they may be able to burn deuterium and lithium they will slowly fade as brown dwarfs. On the high mass end there may be an physical upper limit to the masses of stars. As stars become more luminous radiation pressure can compete with accretion. This is the Eddington limit. Radial pulsations in massive stars may be unstable. A couple of massive stars in the Milky Way have inferred masses up to $\sim 200 \mathrm{M}_{\odot}$, but the most massive star with a reliable measurement, via analysis of its binary system, is WR 20a with a mass of $83 \pm 5 \mathrm{M}_{\odot}$ (Bonanos, et al. 2004).

Due to the same lack of understanding of star formation which plagues predictions of the IMF there is currently no accepted upper limit on stellar masses. The IMF is most commonly defined over the mass interval $0.1<M / M_{\odot}<120$. However some investigators use minimum and, more frequently, the maximum stellar masses as free parameters when fitting an IMF to a stellar population.

In addition to fitting piecewise power laws to the IMF, log-normal functions can also be used. A variable $X$ is $\log$-normally distributed if $Y=\ln X$ has a normal distribution (NIST 2005). The probability density function of the log-normal distribution is given by:

$$
f(x)=\frac{\exp \left[-(\ln ((x-\theta) / m))^{2} / 2 \sigma^{2}\right]}{(x-\theta) \sigma \sqrt{2 \pi}}
$$

where $\sigma$ is the shape parameter, $\theta$ is the location parameter and $m$ is the scale parameter. Miller \& Scalo (1979) were the first to fit an observational measurement of the IMF with a 
log-normal distribution:

$$
\xi(\log m)=C_{0} \exp \left[-C_{1}\left(\log m-C_{2}\right)^{2}\right]
$$

where their three free parameters are $C_{0} \sim 100, C_{1} \sim 1$ and $C_{2} \sim-1$ for their measurement of the galactic field star IMF. The log-normal distribution has several attractive features. It is clearly normalizable and the number of stars formed goes to zero smoothly at both low and high mass without any arbitrary truncation. It is also attractive in that it has fewer degrees of freedom than power laws. A single slope power law has three degrees of freedom, $\Gamma$ and the minimum and maximum masses, while the log-normal distribution has only two ( $C_{0}$ in equation 1.28 is a normalization constant). From an analytical standpoint is far easier to handle. On the downside should there be structure in the IMF over small mass ranges, for which there is some evidence, the log-normal distribution will fail to fit it.

The log-normal distribution has the added appeal that it can arise physically from stochastic processes. Suppose that some function $q$ is equal to the product of $n$ bounded random variables $x_{i}$

$$
q=\prod_{i=1}^{n} x_{i}
$$

Then the logarithm of $q$ is equal to the sum of the logarithms of the individual $x_{i} \mathrm{~s}$

$$
\log q=\sum_{i=1}^{n} \log x_{i}
$$

By the central limit theorem $\log q$ becomes normally distributed as $n \rightarrow \infty$, thus $q$ is $\log$ normally distributed. Figure 1.2 demonstrates this effect. In each plot $q$ is measured 30,000 times as a product of $n$ random numbers uniformly distributed between 0 and 1 . The best fitting Gaussian distribution is over plotted, showing that $q$ converges to a log-normal 
distribution. Ioka \& Nakamura (2002) note that if $\log x_{i}$ is uniformly distributed between 0 and 1 the distribution becomes effectively log-normal when $n \geq 3$. Even for small values of $n$ a distribution can be consistent with a log-normal distribution.

Because of its physical origins the log-normal distribution is the preferred parameterization of the IMF in theory papers.

\subsection{Observational Data}

\subsubsection{Photometry}

Astronomers who work in the optical and near-infrared usually measure flux in units of apparent magnitude

$$
m=-2.5 \log \left(\frac{f}{f_{0}}\right)
$$

The brighter objects have lower magnitudes. A change in five magnitudes is a hundred-fold difference in flux. In $\mathrm{AB}$ magnitudes a flat spectrum with flux of $3.631 \times 10^{-23} \mathrm{Wm}^{-2} \mathrm{~Hz}^{-1}$ corresponds to magnitude zero. The Sun has an apparent magnitude of -26.8 . Venus varies from -4.4 to -2.5 . The faintest stars seen by the naked eye are magnitude +6 to +7 depending on the person. The limiting magnitude of SDSS is around +21 . The Hubble Deep Field goes down to +28 . A convenient mathematical coincidence is that

$$
m=-\frac{2.5}{\ln 10} \ln f+c \Rightarrow d m=-1.086 \frac{d f}{f}
$$

so that magnitude errors are approximately equal to the fractional uncertainty in flux.

The absolute magnitude, $M$, is a measure of luminosity. It is related to the apparent 
magnitude by the distance $d$

$$
m-M=5 \log \left(\frac{d}{10 \mathrm{pc}}\right)
$$

At cosmological distances $d$ is the luminosity distance, a function of redshift $z$, Hubble constant $H_{0}$, matter density $\Omega_{M}$, cosmological constant $\Lambda$ and cosmological deceleration parameter $q_{0}$ which takes into account deviations from flat space time.

The color or color index is the difference of two broadband magnitudes, such as $g-r$ or $B-V$. The color index is the ratio of the fluxes in the two band passes

$$
g-r=-2.5 \log \left(\frac{f_{g}}{f_{r}}\right)+c
$$

where $c$ is a constant. The shorter wavelength magnitude is first by convention. A smaller color index implies that the object is blue- that the flux is greater at shorter wavelengths. A larger color index indicates the object is red.

A spectrum can be converted into broadband magnitudes. The AB magnitude through an arbitrary filter for a spectrum is calculated with the following integral, where $f_{\lambda}$ is the flux of the spectrum per unit wavelength and $S_{\lambda}$ is the total response of the filter and system.

$$
m=-2.5 \log \left(\frac{\int d(\log \lambda) \lambda^{2} f_{\lambda} S_{\lambda}}{\int d(\log \lambda) S_{\lambda}}\right)+40.093
$$

\subsubsection{Filter Sets}

Standardized broadband filters are widely used in astronomical imaging. Parameters for several commonly used filters are found in Table 1.2. The central wavelengths of the filters are given, as are the widths. Figure 1.1 shows the approximate range of some of these filters overlaid on the spectra of stars of several spectral types. The $B, g, V, r$, and $R_{C}$ 
Table 1.2. Properties of Selected Astronomical Filters

\begin{tabular}{cccccc}
\hline \hline Filter & Center $(\AA)$ & FWHM $(\AA)$ & Filter & Center $(\AA)$ & FWHM $(\AA)$ \\
\hline FUV & 1516 & 269 & $R_{c}$ & 6581 & 1576 \\
NUV & 2267 & 616 & $i$ & 7672 & 1535 \\
$u$ & 3557 & 599 & $I_{c}$ & 8059 & 1543 \\
$U$ & 3652 & 524 & $z$ & 9097 & 1370 \\
$B$ & 4448 & 1008 & $J$ & 12500 & 3000 \\
$g$ & 4825 & 1379 & $H$ & 16500 & 3000 \\
$V$ & 5505 & 826 & $K_{S}$ & 21600 & 3300 \\
$r$ & 6261 & 1382 & & & \\
\hline
\end{tabular}

filters span the optical range of wavelengths to which the human eye is sensitive. The $i, I_{C}$, $z, J, H$, and $K_{S}$ filters cover wavelengths in the near infrared, beyond the perception of the eye. The FUV, NUV, $u$, and $U$ filters cover ultraviolet wavelengths. These are also beyond the scope of human sight but at wavelengths shorter than in the optical. Standardized filter sets allow astronomers to be able to compare observations and results from different telescopes and instruments. Many commonly used relationships in the literature are expressed in terms of standardized filters. For instance dust extinction is usually expressed as $A_{V}$, the level at which light in the $V$ band is decreased in magnitudes. The bandpass of a broadband filter may be designed with specific spectral features of astronomical objects in mind or to exploit regions of transparency in the Earth's atmosphere.

Table 1.2 is hardly an exhaustive list of filters. Many filters are instrument specific. There are also narrow band filters which can be used to image an object at a specific 
wavelength. For instance a narrow band filter centered at $6565 \AA$ can be used to image $\mathrm{H} \alpha$ emission from a galaxy which can reveal regions of active star formation. A Fabry-Pérot, etalon is a tunable narrow band filter. This is useful for imaging specific emission lines in galaxies in the Hubble flow where the emission lines are redshifted to wavelengths were no narrow band filter is available. Cutoff filters which block light above or below a certain wavelength are particularly useful in spectroscopy to prevent first and second spectral orders from overlapping on the CCD chip

\subsection{The Sloan Digital Sky Survey}

The data for these project come primarily from the Sloan Digital Sky Survey. The project goal for the SDSS is to image roughly one quarter of the sky in five bandpasses, $u, g, r, i$ and $z$ (Stoughton, et al. 2002) which are described by Fukugita, et al. (1996). Followup spectra are taken based on the photometry. It is estimated that this will result in spectra of about $10^{6}$ galaxies, $10^{5}$ quasars, 30,000 stars and 30,000 serendipitous targets. However, the SDSS is behind schedule so these goals will not be fully met by the end of the project, although an extension to complete observations in the stated footprint is plamed pending funding.

As this project is still in a testing phase only the second data release data are used instead of the full set of observations. The second data release covers $3324 \mathrm{deg}^{2}$ in imaging of which $2627 \mathrm{deg}^{2}$ have been followed up with spectroscopy (Abazajian, et al. 2004). Point sources are $95 \%$ complete to $r=22.2$ although experience shows that photometry of extended objects is reliable only to $r \sim 20.5$. SDSS spectroscopy covers the wavelength 
range 3800 to $9200 \AA$ with $R \sim 1800$. With each data release old calibration issues are resolved and new ones are discovered. The advantage of using a single data release is that the data are homogeneous, having been rereduced with a single version of the pipeline.

The SDSS uses a dedicated $2.5 \mathrm{~m}$ telescope at Apache Point Observatory in the mountains east of Alamogordo, New Mexico. Imaging is taken in drift scanning mode by a camera with 30 CCDs. There are six columns of detectors which contain one of each of the five ugriz filters. Spectroscopic targets are selected from the photometry. There are three main spectroscopic surveys within the SDSS, the quasar sample, luminous red galaxy sample and the main galaxy sample. This project uses the main galaxy sample.

The photometric data set contains well over 100 measured parameters for every object. Among these are four different magnitude types: fiber, PSF, petrosian and model. Fiber magnitudes have a 3 " aperture to match the 3 " fibers on the spectrographs. Originally they were not intended for science but in recent versions of the photometry they are now seeing corrected. PSF magnitudes fit the point spread function to an object to determine the flux and are recommended for point sources. Petrosian magnitudes fit a petrosian profile to the objects and are recommended for the brighter galaxies in the spectroscopic surveys. Model magnitudes model objects with both exponential and de Vaucouleurs profiles and choose the better fit. Model magnitudes are best for extended objects which are nearing the photometry limits. Magnitudes can also be determined from the spectra but the absolnte spectrophotonetry is not as well established as the photometry.

The SDSS uses its own system of photometry based on the inverse hyperbolic sine (Lupton, Gunn \& Szalay 1999). These are referred to either as "arcsinh" magnitudes or 
"luptitudes. The flux of a blank portion of the sky after sky subtraction is zero. However due to Poisson noise the measured fiux has a $50 \%$ chance of being negative. In both the Vega and $A B$ systems negative flux values are mishandled because the logarithm of a negative number is undefined. This results in a loss of information for objects with fluxes near the survey limit. The function arcsinh is defined for all real numbers and is a one-to-one mapping. For large positive numbers the arcsinh becomes proportional to a logarithm. Negative flux values can be recovered from arcsinh magnitudes and quickly approach the AB system above the survey limits. For the bright objects targeted by spectroscopy the difference between luptitudes and AB magnitudes is academic, unless they are $u$ or $g$ band dropouts.

\subsection{The Universality of the Stellar Initial Mass Function}

The process of star formation is still poorly understood theoretically. This leads directly to difficulty in deriving the IMF from basic principles. From the beginning with Salpeter in 1955 the study of the IMF has been driven by empirical measurements. In addition to the theoretical challenges, unfortunately, all observational measures of the IMF are particularly challenging and involve many uncertainties. As a result there are large observational uncertainties in the IMF as well.

Currently astronomers studying star formation are embroiled in a debate over whether or not the IMF is universal. Does the IMF change as a function of envirommental conditions such as cloud temperature, metallicity, density or mass, or is it the same everywhere regardless of the properties of the cloud? A first thought would be to side strongly 
with variations as the Jeans mass in equation 1.19 is clearly a function of temperature, density and mean molecular weight. However observations do not bear this out. As of yet there is no definitive evidence of systematic variations. Measures of the IMF spamning wide variations of environments using an equally broad range of methods are consistent with each other.

On the other hand there is as yet no definitive evidence ruling out systematic variations. It could be that variations exist, but are on a similar scale with the measurement, errors. Some mass regimes have been too poorly studied to make any conclusions. Scalo (2005) points out that "there is currently little basis for assigning a given average, let alone universal, value" to the IMF over the intermediate range from 1 to $15 \mathrm{M}_{\odot}$. Likewise for brown dwarfs the IMF can only be measured directly in the solar neighborhood. It is also possible that a universal IMF can be consistent with a wide range of environments yet fail in extreme circumstances.

While many astronomers may take a universal IMF for granted, the jury is still out.

This is particularly vexing as an understanding of the IMF is essential in a broad range of astrophysical topics. Obviously the IMF is of paramount importance in the study of star formation. Pavel Kroupa writes that the discovery of systematic variations in the IMF would be the "Rosetta Stone" for star formation (Kroupa 2001). Working backward from the IMF, theorists would have a framework to check their ideas against where currently there are few clues.

The IMF is also of great importance to the study of galaxy evolution. Much of 
what is known about the evolution of galaxies comes from comparing observations with models of the stellar populations of galaxies. One of the main inputs into these spectral synthesis models is the assumed stellar IMF. If the IMF varies with environment, or over cosmic time, calculated values of star formation rates, mass to light ratios and dark matter content of galaxies could be incorrect. IMF variations could have a similar effect on models of chemical enrichment. Some measurements of the cosmological deceleration parameter $q_{0}$ are also IMF dependent.

Even the null hypothesis, that the IMF is universal, is worth studying if the error bars can be further constrained. This section focuses on evidence for variations in the IMF from both observations and theory.

\subsubsection{Observational Evidence}

Observational measurements of the IMF can be divided into two basic categories: those relying on counts of individual stars in a given population and those from modeling the integrated light from more distant stellar populations.

\section{Star Counts}

Salpeter's original measurement of the IMF was based on a census of stars in the solar neighborhood. This census is a stellar luminosity function- the number of stars observed in each range of luminosity. Salpeter noted that this luminosity function should depend on three factors: the star formation rate (SFR) as a function of time or star formation history ( $\mathrm{SFH})$, the evolution of stars off the main sequence as they exhaust their fuel, and the IMF. 
Construction of the luminosity function begins with the measurement of the apparent magnitudes of a large number of stars. The apparent magnitudes need to be converted to luminosity using distance measurements. For measurements of the field stars in the solar neighborhood distances must be measured for individual stars. This is most frequently done using trigonometric parallaxes, although Miller \& Scalo (1979) mention a few distance estimators used in the past.

As stars evolve off the main sequence it becomes more difficult to infer what their initial masses were due to mass loss, especially on the asymptotic giant branch, and uncertainties in models of stellar evolution. In order to avoid this uncertainty the main sequence luminosity function $\dot{\Psi}\left(M_{P}\right)$, where $M_{P}$ is the absolute magnitude in the bandpass measured, is used in place of the full luminosity function.

Measuring $\Psi\left(M_{P}\right)$ requires the differentiation of main sequence stars from post main sequence stars. For example an A0 V star has $M_{V}=0$, but so do K2 III starsboth contribute to luminosity function. Salpeter (1955) and Miller \& Scalo (1979) make this correction by multiplying their luminosity functions by the fraction of stars that are on the main sequence at a given luminosity. The main sequence fractions are determined by spectroscopic studies of a magnitude limited sample of stars and then assumed to be global. This is less than ideal. It assumes that the Sun occupies a region which is representative of this part of the galaxy as a whole. There is also significant variation across these measurements for the most luninous stars. A far preferable method is to determine the luminosity class for individual stars. If color information (i.e. $B-V$ ) is available in addition to the absolute magnitudes luminosity classes can be sorted out. 
An intermediate step is to determine the present day mass function $\Xi(m)=$ $\mathrm{d} N / \mathrm{d} m$, which has a notably similar form to the IMF. $\Xi(m)$ is the relative number of stars in the mass interval $m$ to $m+\mathrm{d} m$. The present day mass function is determined by the following

$$
\Xi(m)=-\Psi\left(M_{P}\right)\left(\frac{\mathrm{d} m}{\mathrm{~d} M_{P}}\right)^{-1}
$$

where $\mathrm{d} m / \mathrm{d} M_{P}$ is the main sequence mass-luminosity relation. The mass-luminosity relation converts the luminosity function to a mass function. The mass-luminosity relation is determined from main sequence visual, eclipsing and spectroscopic binaries from which the mass can be determined. The luminosity of a main sequence star is not a function of mass alone. It also depends on the metallicity, spin vector and main sequence age. Stars with lower metal content have lower opacity and are therefore brighter at a given mass. The internal pressure of a star is reduced by increased rotation rates which in turn makes them dimmer (Kroupa 2002).

Stars will brighten by about 1 magnitude over their lifetime on the main sequence. For low mass stars which are in their relative infancy this will be a minor effect as they have not yet brightened significantly. However it is a significant effect for stars with lifetimes shorter than the age of the galaxy. The luminosity of these stars will be on average 0.5 magnitudes brighter than they were at ZAMS. This effect must, be taken out using the main sequence brightening correction (Miller \& Scalo 1979).

The IMF can be calculated from the present day mass function by the following:

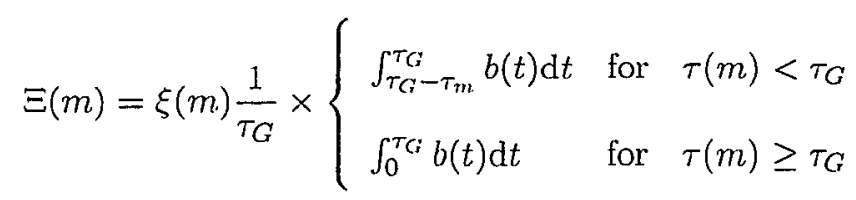


where $b(t)$ is the SFH with the normalization condition $\left(1 / \tau_{G}\right) \int_{0}^{\tau_{C}} b(t) \mathrm{d} t=1, \tau_{G}$ is the current age of the galaxy and $\tau(m)$ is the main sequence lifetime of a star of mass $m$ (Kroupa 2001). In this expression $\xi(M)$ is the time averaged IMF. For stars with lifetimes longer than the current age of the galaxy $\Xi(m)=\xi(m)$. Equation 1.37 illuminates the effect of the SFH on the observed IMF. Another assumption inherent in equation 1.37 is that the IMF is at least nearly constant over time. Miller \& Scalo (1979) suggest there is no compelling observational evidence to indicate this is a bad assumption.

There are a host of other complications to measuring the IMF from counts of main sequence field stars. In a magnitude limited sample luminous stars are overrepresented. This is called the Malmquist bias. The Malmquist bias arises because brighter stars can be seen at greater distances. In a magnitude limited sample will detect luminous stars will be detected over a greater volume of space than fainter stars. For example, a magnitude limited sample with a limiting magnitude of $V=6$ will find that $12 \%$ of stars have $-2.5<M_{V}<-1.5$. However the true value from a volume limited sample is $0.1 \%$ (Binney \& Merrifield 1998). The luminosity function needs to be corrected for the Malmquist bias.

In addition to the Malmquist bias, there is also the Lutz-Kelker bias (Lutz \& Kelker 1973). The Lutz-Kelker bias results in measured luminosities that are systematically too large. Because there is uncertainty in the value of a measured trigonometric parallax for a given actual parallax half of the measured parallaxes will be too high, while the other half will be too low. This effect would tend to cancel itself out, but parallax is a function of distance. Assuming a uniform distribution of stars there are more stars in each more distant spherical shell. Therefore more stars of lower parallax are scattered into bins of 
higher parallax than vice versa. The result is an systematic underestimate of the true distance to a population of stars. This effect occurs at all values of the parallax and is not fixed by increasing the value of the limiting parallax. The effect is a function of $\sigma / \pi$ not $\pi$ as one might assume, where $\sigma$ is the measurement error and $\pi$ is the value of the parallax.

High mass stars are more often found on the galactic disk while low mass stars are less confined to the disk. It is therefore important to look perpendicular to the disk to ensure that low mass stars are not underrepresented in the present day mass function (Miller \& Scalo 1979).

Another significant problem is the existence of unresolved binaries. In this case two or more stars near each other can be mistakenly counted as one thus underestimating the number density in a given magnitude, and therefore mass, bin. This problem is a function of distance as a larger fraction of binary stars will be unresolved at greater distances. $42 \% \pm 9 \%$ of main sequence $M$ stars (Fischer \& Marcy 1992) and $43 \%$ of main sequence $G$ stars (Duquennoy 1991) are primary stars in multiple star systems. These are both lower limits as some companions may have eluded detection. As roughly half of stars are in multiple systems it has a potentially large effect on the observed luminosity function.

Malkov \& Zinnecker (2001) investigated the severity of this problem using Monte Carlo simulations. Binary pairs were chosen at random based on a assumed underlying single-star IMF. As light from the primary star will dominate the total light from the system, lle secondary can be rendered invisible. This is a particularly large problem for lower mass stars as it is unlikely that they will be the primary star in a random pairing of stars. Because of their longer lifetimes, lower mass stars can be paired with stars that 
have already evolved off the main sequence and are thus thrown out of the main sequence luminosity function altogether. The secondary star IMF will always have a steeper power law slope than the observed primary star IMF. This suggests that star count measures of the IMF will systematically underestimate the slope of the true underlying IMF.

To first order Salpeter's original mass function is still widely in use. Although it is was determined from stars in the mass range $0.4<\mathrm{M} / \mathrm{M}_{\odot}<10$ it is often extended to cover the range $0.1<\mathrm{M} / \mathrm{M}_{\odot}<120$ with a slope $\Gamma=1.35$. More modern field star $\mathrm{IMF}$ measurements include Miller \& Scalo (1979),

$$
\Gamma=\left\{\begin{array}{ccc}
0.4 & \text { for } & 0.1 \leq m / \mathrm{M}_{\odot} \leq 1 \\
1.5 & \text { for } & 1 \leq m / \mathrm{M}_{\odot} \leq 10 \\
2.3 & \text { for } & 10 \leq m / \mathrm{M}_{\odot}
\end{array}\right.
$$

and Kroupa, Tout, \& Gilmore (1993)

$$
\Gamma=\left\{\begin{array}{ccc}
0.3 & \text { for } & 0.08 \leq m / \mathrm{M}_{\odot} \leq 0.5 \\
1.2 & \text { for } & 0.5 \leq m / \mathrm{M}_{\odot} \leq 1.0 \\
1.7 & \text { for } & 1.0 \leq m / \mathrm{M}_{\odot}
\end{array}\right.
$$

Figure 1.3 shows some IMFs graphically. There is evidence to suggest that the solar neighborhood is deficient in massive stars and therefore field star observations systematically underestimate the number of high mass stars formed (Kennicutt, Tamblyn, \& Congdon 1994).

Due to the dynamics of star clusters and the Milky Way as a whole, ficld stars come from a mixture of many star formation events at various times in the past. As such the field star IMF is an average IMF. While a useful benchmark it gives little insight into possible variations by itself. 
The stellar census technique used for field stars can also be applied to clusters of stars. Open clusters are more useful than globular clusters as they are young enough to have massive stars and close enough to observe lower mass main sequence stars.

There are several advantages to using open clusters for IMF measurements (Phelps \& Janes (1993), Scalo (2005)). In an open cluster all of the stars are at the same distance and have the same age and metallicity. This eliminates the need to assume a timc-independent IMF and make birthrate corrections, the latter of which is especially problematic for IMF measurements in the 1 to $2 \mathrm{M}_{\odot}$ range. Because of their long lifetimes field stars with masses $\lesssim 2 \mathrm{M}_{\odot}$ can migrate large distances from their birthplaces. In a cluster the stars are all still near the place of their birth. The luminosity function can be directly converted to the IMF below the main sequence turnoff provided that the range of formation times is less than the cluster age.

Unfortunately there are a number of complications associated with cluster IMF measurements (Phelps \& Janes (1993), Scalo (2005)). Clusters have relatively few stars which leads to poor statistics in the luminosity functions and thus IMFs of individual clusters. Also contributing to poor statistics is the small number of clusters that are both young enough to have a large range of masses and close enough that the limiting magnitude does not affect the detectability of low mass stars. Field stars along the line of sight can make cluster membership difficult to determine. If the star formation history of the cluster deviates from a single burst evolution corrections may be necessary.

Another complication of using clusters is that there is evidence of mass segregation-that more massive stars either form at or fall into the center of clusters. Using HST/WFPC2 
images of the young open cluster NGC 330 in the SMC Sirianni, et al. (2002) found that the measured value of $\Gamma$ in the mass range $1 \lesssim M / M_{\odot} \lesssim 6$ becomes steeper at greater distances from the cluster core. In the core of cluster $\Gamma \sim 1.5$, consistent with the Salpeter value, and roughly constant. Outside the core $\Gamma$ steepens roughly linearly with distance and reaches $\Gamma \sim 2.8$ by a distance of three core radii.

This steepening is due to a deficit of massive stars rather than an excess of lowmass stars. The density of $1.5 \mathrm{M} / \mathrm{M}_{\odot}$ stars falls by a factor of 4 , and by a factor of 20 for $5.0 \mathrm{M} / \mathrm{M}_{\odot}$ stars.

An excess of massive stars in the core can arise from dynamical evolution of the cluster. Over time equipartition of energy can lead to low mass stars taking part of the kinetic energy of higher mass stars, causing the higher mass stars to fall deeper into the potential well of the cluster. However in the case of NGC 330 the timescale for this to happen is $500 \mathrm{Myr}$ which exceeds by factor of 20 the $30 \mathrm{Myr}$ age of the cluster suggesting that a significant portion of the mass segregation is primordial in nature.

The other possibility is that the mass segregation is due to IMF variations. In this case the higher gas densities at the center of the cluster lead to a higher accretion rate which facilitates the formation of stars with higher masses.

It is difficult to compare cluster IMF results from different authors due to varying data reduction and models used. Fortunately there are a few authors who have measured the IMFs of several clusters with the sanne techniques. The variation in $\Gamma$ values in the mass range from 1 to $15 \mathrm{M}_{\odot}$ for individual authors is as large for individual authors with self-consistent techniques as it is across multiple authors using different techniques (Scalo 
2005).

Phelps \& Janes (1993) measured IMFs for 8 individual clusters. Combining their results they found a mean value $\Gamma=1.40 \pm 0.13$ over the mass range $1.4<\mathrm{M} / \mathrm{M}_{\odot}<7.9$ consistent with Salpeter's value of $\Gamma=1.35$. However two of the 8 clusters have IMF slopes significantly different from the mean. For NGC 663 they found $\Gamma=1.06 \pm 0.05$ over the mass range $1.2<\mathrm{M} / \mathrm{M}_{\odot}<12$. For NGC 581 they found $\Gamma=1.78 \pm 0.05$ over the same mass range. The authors believe this difference to be real because NGC 581 and NGC 663 are both well sampled as they are relatively populated, and they have the same ages and physical size.

Sanner \& Geffert (2001) also measured IMFs for several open clusters. Their measured slopes varied from $\Gamma=0.69 \pm 0.63$ to $\Gamma=2.27 \pm 0.70$ in the mass range $1.1 \lesssim \mathrm{M} / \mathrm{M}_{\odot} \lesssim 6$, with an average of $\Gamma=1.65$. Their data came from the Tycho-2 catalog so they were able to use proper motions to determine cluster membership as opposed to Phelps \& Janes method of estimating the contamination from measurements of nearby field stars. However their limiting magnitude of $V_{T} \sim 11$ led to much poorer statistics and thus more uncertain values of $\Gamma$.

Studying the high mass end of the IMF is in general difficult because the highest mass stars experience supernovae within $3 \mathrm{Myr}$ of their birth. In the case of clusters it is necessary to observe a cluster at an extremely young age at a distance where individual star counts are still possible. One known cluster neeting those criteria is the Arches Cluster (Figer, et al. 2002). The Arches Cluster is the densest and one of the most massive young clusters in the galaxy, containing at least $160 \mathrm{O}$ stars. The age is estimated to be $2.5 \pm 0.5$ 
Myr.

Figer (2005) measure a best fit $\Gamma=0.90$ over the mass range $10<\mathrm{M} / \mathrm{M}_{\odot}<120$. They also provide a conservative estimate for the upper mass limit of $150 \mathrm{M}_{\odot}$ for stars based on Monte Carlo simulations of their photometry of the Arches Cluster. Their measured mass function truncates at $130 \mathrm{M}_{\odot}$, even though higher mass stars should still be observable given the cluster age.

The high mass end of the IMF is also heavily affected by stochastic processes. Kroupa (2001) made cluster models by selecting the masses of the stars randomly from a universal IMF mass distribution. Cluster membership ranged from $10^{2}$ to $10^{6}$ stars. The IMF was then measured for these clusters using the assumption that the masses of stars could be determined exactly. Even with this generous assumption there was a large amount of scatter in the IMF slopes of the small clusters. In the case of $10^{6}$ stars the true IMF was recovered exactly. Based solely on random sampling of masses Kroupa concluded that the standard deviation of $\Gamma$ across many cluster IMF measurements is $\sim 1$, even if the underlying IMF is truly universal.

Another way in which stochastic processes can complicate studies of IMF variations in stellar clusters has to do with the order in which the stars in the cluster form. $O$ and $\mathrm{B}$ stars produce ionizing photons and cosmic rays which affect the surrounding nebula. However the probability of creating one of these massive stars is comparatively small. If one of these massive stars happens by chance to form first it maly drive up the nebular. temperature and depress the formation of less massive stars compared to regions without: massive stars (Robberto, et al. 2004). 


\section{Integrated Light}

Stellar count methods have a serious limitation. They can only be used for stellar populations which are close enough to resolve the individual stars. They are of diminishing usefulness as a function of distance as the lowest mass stars become too faint to detect. In a search for systematic variations in the IMF due to environmental conditions it is necessary to investigate the widest possible range of conditions. There is a far greater range of environments across the populace of galaxies in the local universe than can be found within the Milky Way alone so there is a strong motivation to probe the IMF in other galaxies.

The techniques for studying the IMF in other galaxies differ greatly from those of resolved stellar populations in our galaxy. Instead of counting individual stars, the integrated light of the entire stellar population is looked at all at once. From the integrated light IMF indicators can be used to decipher the underlying IMF of the galaxy. There are both advantages and disadvantages to this change in tactics.

In addition to the increased access to a greater variety of environments integrated light techniques eliminate many of the biases of star counting methods. Corrections for Malmquist and Lutz-Kelker biases as well as the structure of the disk of the galaxy are not necessary. The need to differentiate between main sequence and post main sequence stars vanishes and the problems arising from multiple star systems are eliminated.

On the down side integrated light techniques are even more model dependent and therefore can only be as good as the models on which they are based. These models can be degenerate. It is difficult to disentangle the effects of age, metallicity, star formation history and IMF. Individual IMF indicators provide only weak constraints on the IMF so they are 
better used in tandem.

Leitherer (1998) divides integrated light techniques into three main categories: those that measure the mass-to-light ratio to determine the lower mass cutoff of the IMF, those that measure the IMF slope above $10 \mathrm{M}_{\odot}$, and those which attempt to find the high mass cutoff from the ionizing radiation field. What follows is a sampling of studies using a range of integrated light indicators and the insight provided into IMF variations or lack thereof.

Kennicutt (1983) compared the $\mathrm{H} \alpha$ equivalent width to the $B-V$ color index for a sample of galaxies to constrain the slope of the IMF for higher stellar masses. The $\mathrm{H} \alpha$ equivalent width compares strengths of the $\mathrm{Ho}$ emission line to the continuum around the line. The equivalent width is the width of the continuum around an emission line whose integrated flux is equal to the flux in the emission line. The equivalent width has the advantage over line flux measurements in that it is minimally affected by dust and flux calibration.

The strength of the $\mathrm{H} \alpha$ line is closely related to the number of massive stars present under the assumptions of Case B recombination (Osterbrock 1989). Because all $\mathrm{O}$ and $\mathrm{B}$ stars are young they are often still in the regions of neutral hydrogen from which they formed. Only massive $\mathrm{O}$ and B stars produce appreciable UV flux which ionizes the gas surrounding them. In Case B recombination the clouds are dense so any photon emitted from an $n^{2} P$ to $1^{2} S$ state is innmediately reabsorbed, populating the $n^{2} P$ state in ancther atom. In addition to re-emitting a Lyman photon, there is also a chance that a transition to $n \geq 2$ will occur. So after several scattering events every Lyman photon is converted into 
lower series and $2^{2} S \rightarrow 1^{2} S$ continuum photons which experience lower optical depths and can escape the gas cloud. As the transition probabilities, although weakly temperature and density dependent, can be calculated the ratio of ionizing photons from stellar photospheres to $\mathrm{H} \alpha$ flux is well determined.

The inferred UV flux can be converted to a SFR for stars with masses greater than about $10 \mathrm{M}_{\odot}$. The massive star SFR is only weakly a function of the IMF as the range of masses is small. A total SFR can also be determined although it is strongly dependent on the assurned IMF. It is important to remember that these SFRs depend on the Case B assumption. Lyman leakage is when some fraction of the ionizing photons escape the cloud before being converted to photons of lower series. The extent to which this happens depends on the densities and geometries of the stellar nurseries. Thus the calculated SFRs are lower limits to the true SFR.

The $\mathrm{H} \alpha$ equivalent width combines the $\mathrm{H} \alpha$ emission from massive stars with the red continuum primarily arising from red giant stars in the $0.7-3 \mathrm{M}_{\odot}$ range. The relationship between the $\mathrm{H} \alpha$ equivalent width and a broad band color index, namely $B-V$, is sensitive to the slope of the IMF between 1 and $50 \mathrm{M}_{\odot}$. Based on comparisons with spectral synthesis models Kennicutt (1983) found that the slope of the IMF in his sample of galaxies was consistent with $\Gamma=1.5$, and thus the Salpeter slope. It also effectively ruled out the MillerScalo IMF as that IMF failed to produce enough $\mathrm{H} \alpha$ emission to be consistent with the daba. The lack of any information regarding the annount of dust extinction in individual galaxies was a major barrier to more detailed conclusions.

The method was revisited by Kennicutt, Tamblyn, \& Congdon (1994) with an 
expanded sample of galaxies and improved stellar evolution models. They also noted a systematic calibration error of $16 \%$ in the Kemnicutt (1983) data which was corrected. Repeating the analysis they found the IMF slope above $1 \mathrm{M}_{\odot}$ consistent with $1.35 \lesssim \Gamma \lesssim 1.5$ and concluded that the solar neighborhood is deficient in high mass stars by a factor of 1.5 to 2.5. Both Kennicutt measurements rely on the assumption that the IMF is time independent, as the $\mathrm{H} \alpha$ equivalent width compares the massive stars forming today to the less massive stars formed during much earlier epochs of star formation.

Baldry \& Glazebrook (2003) investigated the form of the IMF using galaxy luminosity densities from the ultraviolet to near-infrared. Galaxy luminosity densities for local $(z \sim 0.1)$ galaxies were estimated through analytic integration of the Schecter function for each bandpass. The luminosity densities form a luminosity-weighted average galaxy spectrum with $R \sim 6$. Spectral synthesis models were fit to this "cosmic spectrum" to measure the slope of the IMF between 0.5 and $120 \mathrm{M}_{\odot}$. For masses less than $0.5 \mathrm{M}_{\odot}$ it was assumed that $\Gamma=0.5$.

This method, like all methods which rely on spectral synthesis models, suffers from a number of uncertainties including chemical evolution, dust attenuation, and the synthetic. spectra themselves. However, Baldry \& Glazebrook used a novel approach to break the IMF/star formation history degeneracy. They assumed that the IMF is universal. Should a universal IMF exist it must apply to a cosmic spectrum. While there are large uncertainties in cletermining the SFHs of individual galaxies the cosmic SFH is much better determined. Metallicity and recent starbursts in individual galaxies make it difficult to determine the age of stellar populations. But the cosmic SFH is measured by the evolution of SFR indicators 
with redshift. While an IMF must be assumed to translate the evolution of SFR indicators into a SFH, a relative SFH can be found if the same indicator is used at all redshifts.

This results in a best fit $\Gamma=1.15 \pm 0.2$ which is shallower but agrees with the Salpeter IMF. While their results are consistent with a universal IMF they do not rule out variations. They also found that $\Gamma<1.7$ at the $95 \%$ confidence level which rules out the Scalo (1986) solar neighborhood IMF as being universal. They also found $\Gamma=1.2 \pm 0.3$ comparing the $\mathrm{H} \alpha$ luminosity density to the $r_{0.1}$ band luminosity density.

A mystery exists in the high mass IMF of starburst galaxies. Starbursts appear to be missing high mass stars. In a burst of star formation the number of massive stars is higher than usual which heats the ISM and intergalactic medium. They also enrich the ISM in supernova events. High mass stars can also expel interstellar gas through high stellar winds.

Bernlöhr (1992) analyzed the starburst in M 82 using evolutionary models of stellar populations. Model predictions were compared to the data for the lower limit of the bolometric luminosity, the ionizing photon flux, $K$ magnitude, CO index and the [O III] $/ \mathrm{H} 53 \alpha$ and $[\mathrm{N} \mathrm{III}] / \mathrm{H} 53 \alpha$ far infrared emission line ratios. The $\mathrm{CO}$ index is the strength of the $\mathrm{CO}$ absorption bands at around $2.3 \mu \mathrm{m}$. For a given spectral type the strength of the $\mathrm{CO}$ absorption increases with luminosity class. In general absorption becomes stronger as stellar temperature is decreased, surface gravity is increased and metallicity is increased. The CO inclex is an indicator of the duration of a starburst. The far infrared line ratios are particularly sensitive to the upper end of the mass function. Bernlöhr used the models to investigate truncations of the IMF at both the high and low mass end. An upper mass 
cutoff of $30 \mathrm{M}_{\odot}$ is the best fit, and the IMF is biased toward massive star's either via a lower mass cutoff of around $2 \mathrm{M}_{\odot}$ or a change in $\Gamma$.

Rieke, Rieke \& Tamblyn (1993) did their own modeling of the M 82 starburst. They concluded that the near-infrared spectrum of M82 is dominated by red supergiants, not metal rich red giants. Their models found that the IMF at the high end does not need to be different from that locally, but below a few solar masses the IMF must be truncated or at least heavily suppressed.

Rigby \& Rieke (2004) explore the use of mid-infrared line ratios as stellar temperature diagnostics, and thus IMF indicators. The He I $2.06 \mu \mathrm{m} / \mathrm{Br} 10$ ratio has simple recombination physics and is largely independent of extinction which makes it an accurate diagnostic of the effective temperature of the ionizing continuum. Unfortunately these lines are weak and can only be observed for nearby galaxies. It can, however, be used to calibrate other line ratios for use at large distances. The emission line ratio [Ne III] $15.6 \mu \mathrm{m} /[\mathrm{Ne}$ II] $12.8 \mu \mathrm{m}$ is extremely insensitive to extinction and is a homonuclear rare gas which makes it free from the effects of chemical abundance and dust depletion.

They find that the mid-infrared line ratios can be matched by models with IMFs truncated at $40-50 \mathrm{M}_{\odot}$. However they give an alternative explanation. It may be that in starburst galaxies the massive stars spend the bulk of their lifetimes deep inside dense, highly extincted clouds similar to the ultracompact H II regions of the Milky Way. The extinction to these regions could be as large as $A_{V} \sim 50$. This makes the malssive stars invisible in the optical and near and mid-infrared.

Low surface brightness galaxies (LSBs) also provide possible evidence for non- 
standard IMFs. Schombert, et al. (1990) lists some characteristics of LSBs. They have low stellar luminosity in the disk and fragmented spiral structure, although some have bulges. They exhibit high HI gas to light ratios $\left(M_{\mathrm{Hl}} / L_{B} \sim 1.5\right.$ compared to $\sim 0.5$ for typical star-forming spirals), unusually blue $B-V$ colors given a paucity of star-forming regions and sparse low luminosity HII regions.

The mass-to-light ratios of LSBs can be explained by fading of the stellar population between periods of star formation. Given the blue colors, however, it is difficult to envision such a scenario. The colors are consistent with a stellar population comprised largely of $\mathrm{A}$ and $\mathrm{F}$ stars, but high surface brightness star formation regions are not observed. Schombert, et al. (1990) made radio measurements for six LSBs and failed to detect $\mathrm{CO}$ in any of them. Their most probable explanation is low metal abundance in the LSBs has led to nonstandard $\mathrm{CO} / \mathrm{H}_{2}$ ratios. However it may also indicate that a lack of cold molecular gas leads to weak star formation. It is also possible that a truncated IMF and a constant star formation rate could explain the large number of $A$ and $F$ stars and the lack of $O$ and $B$ stars. This scenario would reduce the amount of metals produced, raise the mass-to-light ratios as more mass would be in low mass stars. In this case stars would be forming in warm, low density HI regions instead of cold molecular clouds which would suppress high mass star formation. If for some reason this happens in LSBs the IMF would be very different from that in galaxies with more normal surface brightness.

Lee, et al. (2004) also explore the possibility that high mass-to-light ratios in LSBs result from variations in the IMF. They used stellar population models to find that an IMF with $\Gamma=2.85$ over the range $0.1<\mathrm{M} / \mathrm{M}_{\odot}<60$ could fit the $B-V$ and $B-I$ colors of 
their 7 LSBs.

Schombert, et al. (1990) comment that LSBs may provide unique insight into galaxy formation. Many LSBs have normal bulges which suggests that their star formation was normal in the distant past. The disks of LSBs are rich in HI but may lack molecular gas. Most LSBs are found in sparsely populated regions of the universe. Their loneliness and the fact that they still posses a lot of HI gas may suggest that they have lived their lives free of interactions with other galaxies and could be rare laboratories for studying galaxy formation in the absence of external perturbations.

Meyer \& Greissl (2005) suggest that in galaxies experiencing strong starbursts it may be possible to detect low mass pre-main sequence stars. Under normal circumstances the $K$ band light of an integrated stellar population will be dominated by post main sequence red giant stars. The contribution to this light from the main sequence will also be dominated by high mass stars because the mass-luminosity relationship is very steep $\left(L \propto M^{3}\right)$. However for pre-main sequence stars the mass-luminosity relationship is considerably flatter. If a starburst is observed within the first $10 \mathrm{Myr}$, before massive stars have time to become red giants, they estimate that $7-12 \%$ of the $K$ band light will be due to low mass pre-main sequence stars depending on the IMF chosen. These low mass pre-main sequence stars have unique spectra with strong broad CO absorption bands which may be observed as $2-4 \%$ absorption against the continuum. The $\mathrm{CO}$ absorption feature could potentially give the ratio of low to high mass stars formed in starburst galaxios, but it. also depends on nebular free-free emission in the $2 \mu \mathrm{m}$ continuum as well as possible excess emission from protostellar disks. 


\subsubsection{Theoretical Expectations}

Larson (1973) provides perhaps the simplest theoretical model which qualitatively agrees with empirically obtained IMFs. In his model fragmentation happens in a series of discrete events. At each step every cloud fragment either splits into two pieces of equal mass or remains intact. This model has only three input parameters- the total mass of the cloud $M$, the total number of fragmentation steps $N$ and the probability $p$ that a fragment will split in two during a fragmentation step. In this model the masses of the stars created must be equal to $2^{-n} M$, where $0 \leq n \leq N$ and $n$ is an integer, due to the series of halving events. Fragmentation eventually stops at some minimum fragmentation mass $2^{-N} M$. Therefore assumptions of the minimum mass give the value of $N$. The value of $p$ depends on conditions in the cloud which favor or hinder fragmentation.

When $p=1 / 2$ the number of stars of mass $\mathcal{M}=2^{-n} M$ after $N$ steps is given by the binomial distribution. Thus the resulting IMF when $p=1 / 2$ is a lognormal distribution because the mass bins are logarithmic because every star has a mass which is a multiple of an integer power of 2 and the binomial distribution approaches the normal distribution for large numbers of $N$. When $p=1 / 2$ the IMF is centered on a mass of $2^{-N / 2} M$ with a standard deviation of $\sqrt{N} / 2$ in units of $\log _{2} \mathcal{M}$. For cases when $p \neq 1 / 2$ the IMF is centered at $2^{-p n} M$ with standard deviation $\sqrt{p(1-p) N}$. It is concluded that $p$ must not differ much from $1 / 2$ if this model is valid.

Of the three parameters in Larson's model, the one that is the least constrained physically is the probability of fragmentation. He points out that while fragmentation depends on the Jeans mass, $M_{J}$, it is not the whole story. A non-rotating spherical cloud 
will collapse without fragmenting. It is turbulence and angular momentum which lead to fragmentation. Thus $M_{J}$ only gives insight to the maximum amount of fragmentation possible under ideal conditions for fragmentation, not the actual amount that occurs. It is suggest that increased turbulence will raise the value of $p$ and in turn reduce the mean stellar mass formed from the cloud.

The G dwarf problem is the fact that there are very few metal poor main sequence G stars in the solar neighborhood-far fewer than the number predicted by the closed box model of chemical enrichment of the ISM. A similar problem is the flat age-metallicity relation of nearby stars. Older stars are observed to be slightly less metal rich than younger stars. Larson (1998) suggests that the G dwarf problem can be explained by a time-varying IMF. Most explanations for the $\mathrm{G}$ dwarf problem have involved infall of metal free primordial gas onto the galaxy. However large amounts of primordial gas are not observed and the measured infall rate is too low. An IMF which was biased toward higher masses in the past can explain both the $\mathrm{G}$ dwarf problem and the flat age-metallicity relation.

\section{Metallicity and Opacity}

The opacity of the molecular cloud plays a role in the fragmentation process. During the optically thick portion of collapse the opacity traps heat marking the end of the isothermal collapse and stopping fragmentation. The effects of opacity in optically thin stages is more subtle but nonetheless important. The cooling rate is controlled by the opacity in this regime. While clouds with high opacity will be hotter at high densities they will be cooler at low densities. 
Boss (1988) found fragmentation is insensitive to opacity within a factor of $3-4$, as is the minimum protostellar mass formed, $\mathrm{M}_{\min } \sim 0.01 \mathrm{M}_{\odot}$. They listed four main reasons for this insensitivity. First and foremost is the aforementioned discussion of opacity having opposite roles depending on the density regime. The net effect over the collapse may cancel out. Secondly fragmentation is largely determined at temperatures below $100 \mathrm{~K}$ where the differences in opacities between dust models are the smallest. Analytically, $M_{J} \propto \kappa^{1 / 7}$ (Low \& Lynden-Bell 1976) so it may not be surprising that the dependence on opacity is weak. Lastly they cited the lack of grid points in their models. They do however point out that the opacity could be very relevant for Population III stars or in other instances of extremely low metallicity. In their analysis the opacity came largely from dust grains. At extremely low metallicities there can be no dust and the primary source of opacity becomes $\mathrm{H}_{2}$ molecules.

The lack of metal and dust cooling in the primordial clouds suggests that the temperatures must have been significantly higher than they are today. Stahler (1986) gives two possible outcomes for this scenario. The first is that the clouds fragment at densities similar to present day clouds then the resulting stars will have systematically higher masses. This idea is supported by the fact that no extremely metal poor low mass stars are observed today. This is perhaps the most compelling argument that the IMF must have been fundamentally different in the past than it is today, and thus not universal in time. If on the other hand the clouds contracted to higher densities before fragmenting they could have formed solar mass stars. But even in this case there is a paucity of sub-solar mass stars formed compared to the IMF of today.

The main source of cooling in extremely metal poor environments comes from 
rotational and vibrational transitions in $\mathrm{H}_{2}$. Nakamura \& Umemura (2002) discuss the importance of HD cooling as well in primordial conditions. HD has a finite dipole moment which leads to higher radiative transition probabilities than $\mathrm{H}_{2}$. This makes it an important, coolant under certain conditions in spite of its relative abundance. In their analysis they considered several processes which thermally affect the clouds. H cooling occurs via radiative recombination, collisional ionization and collisional excitation. Line emission by $\mathrm{H}_{2}$ and $\mathrm{HD}$ rotational transitions and $\mathrm{H}_{2}$ vibrational transitions has a cooling effect, as does collisional dissociation of $\mathrm{H}_{2}$. Gas is heated by $\mathrm{H}_{2}$ formation.

Using this more thorough analysis Nakamura \& Umemura (2002) also found that the IMF in primordial conditions is very dependent on the initial density of the cloud, but also on the $\mathrm{H}_{2}$ abundance. In cases where the central density is $\lesssim 10^{5} \mathrm{~cm}^{-3}$ the IMF is split depending on the $\mathrm{H}_{2}$ abundance in the cloud. Above a critical abundance of $\sim 3 \times 10^{-3} \mathrm{HD}$ cooling dominates over $\mathrm{H}_{2}$ and the IMF peaks at $\sim 10 \mathrm{M}_{\odot}$. Below this abundance $\mathrm{H}_{2}$ cooling is dominant and the IMF peaks at $\sim 100 \mathrm{M}_{\odot}$. At high densities the mass of fragments can be reduced to $\sim 1-2 \mathrm{M}_{\odot}$. This leads to a strange IMF which is bimodal, heavily dependent on the initial densities of the cloud and missing sub-solar mass stars. This suggests that the IMF is indeed a function of metallicity, albeit sensitive to it only over very large ranges, as well as cloud density in the low metallicity regime.

There are other reasons to believe that the temperature of star forming clouds was higher in the past. The ambient temperature as a function of rodshift is $T=2.73 \mathrm{~K}(1+$ $z$ ). For redshifts $\geq 1.9$ the background temperature exceeds $8 \mathrm{~K}$, which is the minimum temperature of molecular clouds today (Larson 1998). It stands to reason that the coolest 
molecular clouds in the past must have been warmer than they are today. In addition star formation rates were likely higher in the past increasing the am ount of gas heating due to ultraviolet radiation from massive young stars and cosmic rays from supernova.

\section{Turbulence}

While the effects of turbulence in molecular clouds have long been suspected of influencing star formation, efforts to quantitatively investigate the significance of these effects have only more recently become computationally possible.

Random supersonic motions are observed in giant molecular clouds. Padoan, Nordlund \& Jones (1997) modeled a portion of a molecular cloud as isothermal flows which are randomly ramped up to high Mach numbers. This results in a complex network of shocks with as many as 5 orders of magnitude between the highest and lowest densities found in the cloud. It also leads to a density field with log-normal distribution. They also find that the standard deviation of the linear density field is proportional to the Mach number. In this model it is turbulence that causes the over densities which lead to instability and then gravity takes over. From equation 1.18 it can be seen that during the isothermal portion of the collapse the distribution of Jeans masses is determined by the density distribution. This leads to a log-normal distribution of Jeans masses, which has the attractive feature that the IMF has an exponential cutoff below the most probable mass. This mass is determined to be

$$
M_{J, \text { min }}=0.2 \mathrm{M}_{\odot}\left(\frac{n}{1000 \mathrm{~cm}^{-3}}\right)^{-1 / 2}\left(\frac{T}{10 \mathrm{~K}}\right)^{2}\left(\frac{\sigma_{v}}{2.5 \mathrm{~km} \mathrm{~s}^{-1}}\right)^{-1}
$$

where $\sigma_{v}$ is the velocity dispersion of the gas. This can be thought of as a modified minimum Jeans mass because of the exponential cutoff in the IMF below this value. Its dependence 
on the temperature is stronger than in equation 1.18 where turbulence is not considered. Using ISM scaling relations equation 1.40 collapses to $M_{J, m i n} \simeq 0.1 \mathrm{M}_{\odot}(T / 10 \mathrm{~K})^{2}$, although $\sigma_{v}$ has the effect of broadening the IMF distribution. They were able to match the MillerScalo IMF with a mix of star forming clouds with temperatures from 5-40 K, a reasonable range of cloud temperatures.. Single temperature models are not as successful. Using a. $60 \mathrm{~K}$ cloud temperature, which is reasonable for clouds experiencing strong UV and X-ray background, they found a cutoff mass of $4 \mathrm{M}_{\odot}$ in agreement with observational evidence that starburst regions are deficient in low-mass stars.

Padoan \& Nordlund (2005) suggest that fragmentation is due primarily to turbulence and fragmentations of any size can form regardless of the local Jeans mass. Their emphasis is on the flow properties instead of the fluid properties of the gas. Using supersonic MHD turbulence in isothermal flows they found that gas density in a cloud follows a log-normal distribution with higher moments depending on the RMS Mach number. The power spectrum of velocities in the turbulent cloud is found to be a power law, $E_{v}(k) \propto k^{-\beta}$, where $k$ is the wave number. Their numerical simulations found $\beta=1.74$, but the value of $\beta$ is constrained to be between $5 / 3$ for incompressible turbulence and 2 for pressureless turbulence. This leads to an IMF with $\Gamma=1.33$, and is constrained to be $1.3 \lesssim \Gamma \lesssim 1.5$ within the range of possible $\beta$. For large masses this analysis predicts a universal IMF with $\Gamma=1.33$, effectively equal to the Salpeter IMF, based on the universality of the statistical properties of turbulence. Information regarding the initial conditions is quickly lust due to the chaotic nature of turbulence. Below $2 \mathrm{M}_{\odot}$ the turnover in the IMF is still a function of temperature, density and RMS Mach number of the flow. They also stress the statistical 
nature of the high mass IMF slope. Because star formation at high masses is relatively rare studies of individual clusters with small numbers of stars can appear to have varying IMF slopes for stochastic reasons despite the universality of the underlying physics.

The IMF from denser regions may be flatter, or more top heavy. One possibility is that in denser regions cloud fragments may merge into more massive fragments. Shadmehri (2004) shows through numerical modeling that if the IMF is "naturally" steep with $\Gamma=1.7$ in dense environs it can reach $\Gamma=1.35$ through fragment coalescence in one dynamical time.

Elmegreen (1997) considered molecular clouds with fractal geometry in a hierarchical structure. The cloud is divided into pieces, which are divided into subpieces and so on. Clouds are thought to have fractal geometry due to turbulence. Pieces of the cloud are then chosen at random from any level of the hierarchical structure. The final mass of a star is the sum of the masses of all the subpieces that can trace their origin through splitting of the piece chosen. That piece and all of its subpieces are then removed from the cloud. At the lowest levels the pieces tend to have the smallest mass due to numerous splitting, and at the top masses tend to be large. There are also relatively more pieces in the lower levels. The models are varied by changing the relative probabilities of selecting from each level of the structure. Some models give equal weight to all levels. The best fitting models give added weight to the lower levels. This is because the star formation rate is thought to be higher for higher gas densilies.

Random sampling of the fractal cloud alone leads to $\Gamma=1.0$ at the high end of the IMF. When mass competition and density dependent SFRs are considered the slope 
steepens to $\Gamma=1.3$. These values are independent of the input parameters lending credence to the idea of a universal IMF. The mass of the most massive star in a cluster is also shown to be a stochastic process as well. As the cluster mass increases so too docs the mass of the most massive star. The minimum Jeans mass is seen to play a role in determining the mass at which the IMF turns over.

Chapters 2 and 3 detail a new test of IMF universality using photometric and spectroscopic data from the SDSS.

\subsection{Windows to the Pasts of Galaxies}

To fully understand the nature of galaxies today it is important to know how they formed. In addtion the study of galaxy evolution is full fledged field in of itself. The formation of the first stars, the assembly of mass in galaxies and the rise and fall of quasar activity are intimately intwined with the larger cosmological picture. Developments in cosmology can provide useful insight into galaxy evolution and vice versa.

Unfortunately the time scales associated with galaxy evolution dwarf the entire course of human history. Our comparitively brief lifetimes, and even several generations, provide only a single snapshot of galactic histories. We cannot watch galaxies as they evolve.

To access their youthful secrets we must be clever enough to find windows to the pasts of galaxies. Furthermore we must have the technical wherewithal to peer inside. Many of these windows can be found within the Milky Way. A detailed accounting of the age, mass and metallicity of the stars in the galaxy can yield numerous insights into the past. For example, assuming a closed-box model of the chemical evolution of the galaxy far more 
Sun-like stars should have low metallicities than the observed ratio. This is known as the classical G-dwarf problem (Schmidt 1963) and it tells us that there is a serious flaw in at least one of the assumptions involved: that the galaxy acts as a closed-box, the gas is initially metal free or the IMF is time invariant. The dynamics of distinct stellar populations in the galaxy - the disk, bulge and halo - are the frozen in remnants of past interactions. All known globular clusters are very old. This suggests that the conditions in the past must, have been considerably different given that no new globular clusters are forming today. The number and dynamics of dwarf galaxies and tidal streams orbiting the Milky Way provide information about the possible heirarchical formation of our galaxy.

Beyond the Milky Way we can look at other galaxies to see what our and other galaxies were like in the past. Due to the finite speed of light we do not see distant galaxies as they are today, but rather as they were when the photons began their odyssey across intergalactic space to Earth. For the most distant galaxies known the light landing in our telescopes left home when the the universe was only a seventh of its present age - more than 10 billion years ago. Like nearby galaxies these are only snapshots of one moment in time. There is still no direct connection to before and after. However they provide useful information about what galaxies in general were like at early times. Another limitation of this method is that with increasing distance observations become more difficult due to a reduction in both flux and spatial resolution. At the largest distances only the brightest galaxies can be observed. Little can be gleaned about the pasti of galaxics that went on to be today's dwarfs from this technique.

By good fortune some distant galaxies fall along the line of sight behind massive 
galaxy clusters at intermediate distances. If the geometry is right the extreme mass of the galaxy cluster can act as gigantic magnifying glass by bending the light from the distant galaxy and focusing it at the point of the observer. This byproduct of general relativity is called gravitational lensing. This significantly brightens the distant galaxies allowing studies of young galaxies that would not be possible under normal circumstances. Lyman break galaxies (LBGs) are one specific class of objects in the early universe. There are a. few known examples of strongly lensed LBGs allowing high S/N spectroscopy of individual young galaxies. LBGs are more thoroughly described in $\$ 1.7$.

Another way to look into the past is to search the local universe for remnants left over from the early stages of galaxy formation. Some low redshift emission line galaxies have been observed to have anomalously low metallicities. Big Bang nucleosynthesis produces no elements heavier than beryllium. All heavier elements present today have been created by nucleosynthesis in stars or supernovae (while cosmic ray spallation can also create some metals it is not an appreciable amount). Therefore a galaxy with extremely low metallicity must not have experienced much star formation up to this point in time. While its chronological age is the same as any other galaxy in many ways it has somehow been frozen in time. In terms of galaxy evolution the galaxy is young. Given their close proximity more in depth observations can be carried out than for young galaxies at high redshift. In addtion much lower galaxy masses can be probed in this way. A more intensive introduction to extremely metal poor galaxies is given in $§ 1.8$. 


\subsection{Lyman Break Galaxies}

Lyman break galaxies (LBGs) are simply galaxies with $z>2$ which are experiencing bursts of star formation but are otherwise normal. The name comes from their method of discovery which involves analyzing their colors around the rest-frame ultraviolet spectral discontinuity at $912 \AA$ - the Lyman break. The technique is an important breakthrough in that it is a simple, efficient and reliable method for finding high redshift galaxies. The drawback is that it only targets a specific type of galaxy, namely those with strong current star formation, and does not lead to a holistic picture of galaxies in the early universe.

The history of searches for LBGs reaches back several decades. Based on the observation that elliptical galaxies are comprised of low metallicity population II stars combined with the fact that their stellar populations are very old Baade (1958) deduced that they must have been formed in a single burst of star formation in the distant past. When this observation is combined with the assumption that the large masses of today's luminous ellipticals had already assembled at early times it leads to the conclusion that for some range of time at high redshifts there should be a sizable population of young galaxies undergoing intense star formation. Furthermore the high luminosity associated with such ravenous star formation may be observable in the present day.

The discovery of these high redshift galaxies would be a major breakthrough for galaxy evolution studies. This led to many efforts to predict their appearance and search for them. Meier (1976a) used spectral synthesis models to predict what an LBG spectrum should look like. The positions of LBGs in color space were predicted in Meier (1976b).

The first high redshift galaxies were not found by the Lyman break technique, but 
rather by their strong radio luminosity. Spinrad \& Djorgovski (1984) measured the a redshift of $z=1.82$ for $3 \mathrm{C} 256$. Steidel \& Hamilton (1993) took images to $26-27$ magnitudes in three optical bandpasses of the fields of two very high redshift quasars. This was an attempt to identify, in color space, examples of normal high redshift galaxies. Several targets were identified, and one was spectroscopically confirmed at $z=3.428$ by Giavalisco, Steidel, $\&$ Szalay (1994). This success led successful surveys for LBGs from both the ground (Steidel, et al. 1996a) and HST (Steidel, et al. 1996b). Accompanying spectroscopy revealed 16 and $5 z \sim 3$ galaxies respectively. Shapley, et al. (2003) use spectra of 811 LBGs to create composite LBG spectra.

With a substantial sample of $L B G$ s the bulk properties of the sample can be measured. Giavalisco, Steidel, \& Macchetto (1996) describe the general characteristics of LBGs. Their morphologies are largely compact, suggesting that around $90 \%$ to $95 \%$ of the stars that are being formed are concentrated within an area equal to the size of present day luminous galaxies. They often have low surface brightness regions around the central bright cores. Steidel, et al. (1996a) suggest that the flat UV spectra observed indicate that LBGs are not significantly affected by dust extinction. Both conclude that LBGs represent the formation of the spheroidal component of the progenitors of present day luminous galaxies.

Although LBGs are intrinsically very luminous at their cosmological distances they appear faint, $R \sim 24$ to 25.5 (Shapley, et al. 2003). This results in individual spectra with low $\mathrm{S} / \mathrm{N}$ which often are only good enough for redshift determinations. As such composite spectra must be used for more in depth analysis. The next section details the opportunistic use of gravitational lensing to permit in depth studies of individual members of the high 
redshift universe.

\subsubsection{Gravitational Lensing}

One consequence of the general theory of relativity is that the path of photons can be altered by gravity. Einstein (1936) considered the possibility that light from a distant star could be deflected by the gravity of an intervening star with results similar to a couventional lens. Two geometries were considered, one in which the observer was located perfectly along. the line through the two stars and one in which the observer was a small distance off axis. In the first case a circular ring due to the distant star would circle the intervening star. In the second the distant star would appear as two point-like sources on opposite sides of the intervening stars. However the possibility of observing this phenomenon was disregarded. A chance alignment of two stars precise enough to generate a ring was deemed implausible. Worse, the angular radius of the ring and separation of images caused by objects of stellar masses was too small to be resolved by telescopes.

Zwicky (1937) suggested that gravitational lensing by galaxies would stand a much better chance of being observed - due to the large mass of galaxies the angular separation between lenses and images could be much larger and the larger angular extents of galaxies would be resolvable to large distances. As early as 1937 Zwicky pointed out three key benefits of observing gravitational lensing by galaxies. First it would provide an additional test of the general theory of relativity. Secondly it would allow observations of galaxies at distances much larger than would otherwise be possible. Lastly it could be of great help in determining the masses of galaxies.

Gravitational lensing was first observed in 1979 with the discovery of the double 
quasar 0957+561 A and B (Walsh, Carswell \& Weymann 1979). Two quasars were found only 6" apart. The spectra of the two quasars were found to be at the same redshift and identical. Soon thereafter Young, et al. (1980) found in deep CCD imaging that 0957+561 $\mathrm{A}$ and $\mathrm{B}$ bracketed a galaxy at $z=0.39$, the most luminous member of a galaxy cluster.

An example of gravitational lensing is shown in Figure 1.4. SDSS J100434.91 +411242.8 (Oguri, et al. 2004) is the first quasar found to be lensed by a cluster of galaxies. It is particularly dramatic as all previous lensed quasars had image separations of at most 7". In SDSS J1004+4112 the separation reaches 14.6". In the figure four images of the background quasar at $z=1.734$ are clearly visible around a central galaxy at $z=0.68$. Hubble Space Telescope Advanced Camera for Surveys imaging reveals a fifth image of the quasar (Inada, et al. 2005). Not only does lensing magnify the quasar, but the geometry of the images allows the modeling of the mass and geometry of the lens. This can be used to determine the dark matter content of the cluster.

One way of thinking of gravitational lensing is that a gravitational field can cause the index of refraction of the vacuum to deviate from 1 . The index of refraction of the vacuum is derived in Binney \& Merrifield (1998) and is given by

$$
n=1+\frac{2|\Phi|}{c^{2}}
$$

where $\Phi$ is the gravitational field.

Gravitational lensing by a point mass is a special case of the general problem, but the results are illustrative. The angular deflection of light rays by a point mass is given by

$$
\alpha=\frac{4 G M}{c^{2} b}
$$

where $\alpha$ is the angular deflection and $b$ is the impact parameter of the light rays. Given 
this angle there is a particular impact parameter for which the light rays are bent such that they reach an observer. Incoming light at larger impact parameters will be focused behind the observer and at small impact parameters the focus will be in front. This critical impact parameter is called the Einstein radius and is given by

$$
r_{E}=\sqrt{\frac{4 G M}{c^{2}}} \sqrt{\frac{D_{S} D_{L}}{D_{S}+D_{L}}}
$$

where $D_{S}$ is the distance from the source to the lens and $D_{L}$ is the distance from the lens to the observer. When the source, lens, and observer all lie on exactly the same line the observer will see the source as a brightened ring with a radius equal to the Einstein radius. Objects that lie within $r_{E}$ of the line between the lens and observer will be heavily distorted by the intervening lens. These objects are strongly lensed. Objects that lie outside of $r_{E}$ will be far less distorted and are referred to as weakly lensed.

Gravitational lensing has two important properties. One is that it is achromaticas wavelengths of light are affected by the same amount. Colors and spectra of objects, when converted to the rest-frame, are not altered by lensing. The other important property is that gravitational lensing preserves surface brightness. The angular size of an object can be increased by lensing but the surface brightness stays the same. The increase in angular size does lead to an increase in brightness facilitating observations even though the surface brightness is unchanged.

When designing a survey to find lensed objects it is helpful to have some predictions of how often the necessary alignment of objects are expected to occur. Unfortunately this is a difficult question to answer. Turner, Ostriker, \& Gott (1984) investigated lensing by point masses and galaxies which were modeled as isothermal spheres. Their predictions were that 
Table 1.3. Known strongly lensed LBGs.

\begin{tabular}{cccccccc}
\hline \hline Name & RA & dec & \% & Lens z & Magnification & $r$ & Discovery \\
\hline & & & & & & & \\
MS1512-cB58 & 151422.30 & +363624.8 & 2.72 & 0.37 & $22-40$ & 20.60 & 1993 \\
Bullet Cluster & - & - & 3.24 & 0.30 & $\sim 3$ & $R=22$ & 1999 \\
Cosmic Eye & 213512.73 & -010143.0 & 3.07 & 0.33 & $28 \pm 3$ & 20.3 & 2006 \\
8 O'Clock Arc & 002240.78 & +143113.9 & 2.73 & 0.38 & $12.3_{-3.6}^{+15}$ & 19.22 & 2006 \\
\hline
\end{tabular}

$0.1 \%$ to $1 \%$ of quasars should have double images with mean separation 1 ", lenses with separations larger than $4 "$ will overwhelmingly be due to rich galaxy clusters at redshifts between 0.2 and 0.8 , image separation is not related to the redshift of the source, and that image separations of 30 to 60 " by rich clusters are possible.

\subsubsection{Known Strongly Lensed LBGs}

At present there are four known LBGs strongly lensed by gravitational lensing. All four were found serendiptously in studies of galaxy clusters or interactions. The general properties of these galaxies are shown in Table 1.3. They are described individually in detail below.

\section{MS1512-cB58}

MS1512-cB58 (cB58) was the first strongly lensed I.BG to be discovered. cB58 was found serendipitously as part of the Canadian Network for Observational Cosmology (CNOC) Cluster Redshift Survey (Yee, et al. 1996a), which was designed to measure the velocity field of intermediate redshift clusters and determine their mass-to-light, ratios out 
to large radii. Images in the $g$ and $r$ bands were taken of the galaxy cluster MS1512+36. Those images were used to create a slit mask for multi-object spectroscopy of the field. Upon visual inspection Yee, et al. (1996b) found that the spectrum of cB58 possessed many strong absorption lines but they did not match those usually associated with the optical spectra of intermediate redshift galaxies. From the spectrum the redshift was determined to be $z=2.72$. The images revealed that cB58 was clearly an extended object, locate 6 " from the brightest cluster galaxy.

The object $\mathrm{cB} 58$ was unusual for several reasons. One was the large redshift. Another issue was that cB58 is extended, but most striking was the large luminosity. cB58 was observed to have a apparent magnitude of $V=20.64$ which at the redshift of cB58 corresponds to an absolute magnitude of -26.0 at the rest-frame wavelength of $1500 \AA$ making it the largest non-AGN galaxy known. Yee, et al. (1996b) considered the probability that $\mathrm{cB} 58$ was strongly lensed to be small due to the fact that it was resolved along both axes, it was well fit by exponential surface brightness profiles, no multiple images were observed and there was no evidence of shear in the images.

Analysis of HST imaging by Seitz, et al. (1998) showed that cB58 was indeed strongly lensed by 3.35 to 4 magnitudes by the $z=0.373$ cluster MS1512+36. This rejected the earlier notion that cB58 is exceptionally luminous, rather it is consistent with other $z=3$ LBGs. Lensing models show that only the central parts of the cB58 galaxy are seen. The outer regions are not strongly lensed. As gravitational lensing preserves surface brightness the high, and structureless surface brightness of cB58 is not an artifact. This suggests that the star formation rate is high and that star formation regions are distributed 
across the central parts of the galaxy. In addition to insights about cB58 Seitz, et al. (1998) were able to construct mass maps and measure the velocity distribution of the MS1512+36 cluster as well.

Because of the high magnification of cB58 Pettini, et al. (2000) were able to obtain a high quality spectrum with the Low Resolution Imaging Spectrograph on the Keck I telescope. The blue portion of the spectrum had a $3.0 \AA$ FWHM and $\mathrm{S} / \mathrm{N} \sim 40$ and $2.1 \AA$ FWHM and $\mathrm{S} / \mathrm{N} \sim 15$ in the red. The optical spectrum covering $4300-6020 \AA$ corresponds to 1150 to $1930 \AA$ in the rest-frame ultraviolet at the redshift of cB58. The high quality spectrum reveals a lot of information about the physical nature of cB58. The $\mathrm{P}$ Cygni profiles of the C IV and N V absorption lines are consistent with a Salpeter IMF with a continuous SFH. The metallicity is estimated to be $1 / 4$ of the solar value indicating an epoch of earlier star formation. The dust extinction is measured to be $E(B-V)=0.1-0.3$ depending of the extinction curve assumed. A current SFR of $\sim 40 M_{\odot} \mathrm{yr}^{-1}$ is arrived at from the UV luminosity. The redshifts of the Lyman $\alpha$ emission, H II region emission lines, absorption lines of stellar photospheres and interstellar absorption lines are all slightly different. The relative velocities of these populations point to a large amount of energy being contributed by the starburst leading to a mass outflow rate of $\dot{M} \sim 60 \mathrm{M}_{\odot} \mathrm{yr}^{-1}$. In addition, two intervening $\mathrm{Mg}$ II absorption systems are detected at, $z=0.829$ and 1.339.

Furthermore, Pettini, et al. (2002) used the echelle spectrograph on the Keck II telescope to conduct a high resolution study of the chemistry of eB58. The ISM of eB58 is enriched in elements produced by Type II supernovae $(\mathrm{O}, \mathrm{Mg}, \mathrm{Si}, \mathrm{P}$, and $\mathrm{S})$, but deficient in $\mathrm{N}$ and Fe-peak elements $(\mathrm{Mn}, \mathrm{Fe}$, and $\mathrm{Ni}$ ) produced by intermediate mass stars which 
have longer lifetimes than the progenitors of Type II supernovae. This suggests that a large fraction of the ISM enrichment happened within the last $300 \mathrm{Myr}$. The metallicity of the Type II supernova elements (40\% solar) shows that by the lookback time $17 \%$ of the present age of the universe cB58 had already converted a third of its gas into stars. The fact that cB58 is converting its ISM into stars at such a rapid rate points to the idea that it is on its way to becoming an elliptical galaxy or the bulge of a spiral. In addition the energetics of the ISM of cB58 suggests strong mass loss which could provide an explanation for observations of chemical enrichment in the Lyman $\alpha$ forrest at high redshifts.

Due to its unique brightness as a non-quasar member of the high-redshift universe cB58 has been extensively observed across a range of wavelengths. Teplitz, et al. (2000) obtained near-infrared spectroscopy of cB58 using the Keck II telescope. At these wavelengths the rest-frame optical of cB58 can be observed. The optical spectrum is helpful because studies of nearby galaxies are predominantly conducted in the optical. This allows a more direct comparison of cB58 to nearby galaxies. Teplitz, et al. (2000) determined the metallicity of $\mathrm{cB} 58$ using the well-tested $R_{23}$ method which utilizes the $\mathrm{H} \alpha, \mathrm{H} \beta$, [O II] $\lambda 3727,[\mathrm{O} \mathrm{III}] \lambda 4959$ and [O III] $\lambda 5007$ emission lines. They arrived at a metallicity of $1 / 3$ solar which agrees with the values from spectroscopy of the rest-frame ultraviolet.

Baker, et al. (2001) observed cB58 in the far-infrared at $1.2 \mathrm{~mm}$. Compared to local starburst galaxies cB58 has too red of a spectral slope in the ultraviolet given the ratio of its far-infrared hux to ultraviolet hux. They measured the dust content of eB58 using four different methods. Three of the methods, far-infrared to ultraviolet flux ratio, $\mathrm{H} \alpha$ to $\mathrm{H} \beta$ ratio, and comparison of ultraviolet line spectrum to the ultraviolet to $\mathrm{H} \alpha$ ratio, 
yielded similar results. Only the method using the ultraviolet spectral slope gives a result, that disagrees from the others by a factor of three. This leads to the conclusion that the ultraviolet slope is either not as robust of a measure of starbursts as the far-infrared to ultraviolet flux ratio or that it is more prone to observation systematics.

Nakanishi, et al. (1997) observed the ${ }^{12} \mathrm{CO}(J=3-2)$ emission line in the radio to measure the mass of molecular hydrogen in cB58 but did not detect any emission. Baker, et al. (2004) used the IRAM Plateau de Bure Interferometer to make to map $\mathrm{CO}(J=3-2)$ emission from cB58 - the first detection of molecular emission in any LBG. They measure a current age of the star formation episode of $140 \mathrm{Myr}$ and current SFR of $\sim 24 \mathrm{M}_{\odot} \mathrm{yr}^{-1}$.

All together these results show the power of a singly strongly lensed object from the high redshift universe. The chance alignment of a galaxy with an massive galaxy cluster opens a window into the distant past of galaxies that is otherwise inaccessible observationally. The body of observations across the electromagnetic spectrum point to the conclusion that cB58 is the progenitor of a present day elliptical galaxy or bulge of a spiral that is being seen at a special moment in time when it is quickly converting its ISM into stars while simultaneously chemically enriching the intergalactic medium at a very early time in the universe.

\section{The Bullet Cluster}

Photometry by Thcker, Tananbaum, \& Remillard (1995) found a 12 "arc in the field of 1E 0657-56, colloquially known as the Bullet Cluster. Mehlert, et al. (2001) spectroscopically confirmed it as an LBG. The Bullet Cluster is actually at least two clusters in the process of merging and is one of the hottest X-ray clusters known (Tucker, et al. 1998). 
The X-ray data suggest that the mass within $1 \mathrm{Mpc}$ of the cluster is $2 \times 10^{15} M_{(\mathrm{)})}$.

Spectroscopy reveals that the $L B G$ is at $z=3.24$, which makes it the most distant of the known strongly lensed LBGs. However, given the estimated magnification of 3.3 it is also has the lowest magnification. These two factors together make it more challenging to observe than the others. In addition to the main arc there are four other objects in the field which are spectroscopically identified as LBGs with redshifts from 2.34 to 3.08 with magnifications from 1 to 2.8 and apparent magnitudes from $R=22$ to 24 .

In spite of the fact that all five objects in the Bullet Cluster field are at least a magnitude fainter than the other known lensed LBGs their spectra still reveal some clues about young galaxies. Spectral modeling shows that the light from the galaxies is dominated by stars with temperatures around $25,000 \mathrm{~K}$ and metallicities around $1 / 5$ of the solar value. There is also a strong correlation of C IV with redshift which Mehlert, et al. (2001) interpret as an increase in metallicity with the age of the universe.

\section{The Cosmic Eye}

LBG J213512.73-010143 is a strongly lensed LBG at $z=3.07$ discovered in 2006 (Smail, et al. 2007). It is on the line of sight to the MACS J2135.2-0102, a highly luminous $\mathrm{X}$-ray cluster at $z=0.325$. It is composed of two arcs which encircle a early-type spiral at $z=0.73$. Images of this lens system have the appearance of an eye giving rise to the name "Cosmic Fye." The Cosmic Eye was discovered in 2006 as part of program to identify bright lensed galaxies in X-ray luminous clusters for the purpose of constraining cluster mass distributions. The discovery image was taken with the Advanced Camera for Surveys on the Hubble Space Telescope. The image was the combination of three 400s exposures 
using the F606W filter with a resulting sampling of $0.05^{\prime \prime}$. The Cosmic Eye is quite bright with an apparent magnitude of $r=20.3$.

The lensing model shows that the magnification of the Cosmic: Eye due the combined effects of the cluster at $z=0.33$ and the galaxy at $z=0.73$ is $28 \pm 3$. Spectroscopic analysis of the Cosmic Eye show that it has Lyo in absorption as well as strong absorption in ISM lines. The Ly $\alpha$ absorption is stronger than is usually the case in LBGs. This suggests an $\mathrm{H}$ I column density of $\log N(\mathrm{H} \mathrm{I}) \sim 21.7$ which is around 7 times higher than in $\mathrm{cB58}$. The strength of C IV $\lambda 1550$ absorption relative to the Si IV $\lambda 1440$ absorption indicates that the luminosity weighted stellar population is dominated by early B-type supergiants. This is consistent with a $10 \mathrm{Myr}$ old burst of star formation. The Cosmic Eye is moderately reddened $(E(B-V) \sim 0.4)$ and the SFR is estimated to be $100 M_{\odot} \mathrm{yr}^{-1}$.

\section{The 8 O'Clock Arc}

SDSS J002240.78+143113.9 is the most recently discovered strongly lensed LBG (Allam, et al. 2007). It was discovered in SDSS imaging in 2006. Due to its discovery time in the evening it was named the 8 O'Clock Arc. It was found in a catalog of interacting and merging galaxy pairs images. It is a clear gravitational arc curving partially around the $z=0.38$ luminous red galaxy SDSS J002240.91+143110.4. The galaxies are separated by $4^{\prime \prime}$. Spectroscopic observations reveal that the $8 \mathrm{O}^{\prime}$ Clock Arc is at $z=2.73$. It is the brightest known LBG with an apparent magnitude of $r=19.22$. The lens model suggnests that it has a magnification of $12_{-3.6}^{+15}$. 


\subsection{Extremely Metal Poor Galaxies}

Extremely metal poor galaxies, or XMPGs, are galaxies with low nebular oxygen abundances. As discussed in $§ 1.6$ no atoms heavier than beryllium were created as a result, of the Big Bang. Heavier elements are predominantly formed in the interiors of stars and in the violent explosions at the end or their lifetimes. The metal content of a galaxy can give insights into its age and history. In general galaxies with high metal contents have formed multiple generations of stars. Low metallicity indicates that a galaxy has experienced few or a single episode of star formation. This is not a steadfast rule however. The metallicity of a galaxy can be reduced either by galactic winds expelling metal rich gas or by accretion of metal poor gas from an external source.

XMPGs are potentially very interesting for the reason that they may be experiencing their first episode of star formation. This can provide essential clues for the study of galaxy formation in that it effectively shows how the earliest galaxies formed. In addition they can provide insight into the behavior of low metallicity stars, and possibly Population III stars, which would be of great help to many stellar evolution models. A galaxy currently undergoing its first episode of star formation would be mysterious in of itself. How did it avoid forming stars when most other galaxies were doing so over the full age of the Universe? Why is it starting to form stars now?

To discuss XMPGs it, is useful to have a clear definition of what they are. Kunth \& Östlin (2000) define XMPGs to be galaxies with metallicities less than $1 / 10 Z_{\odot}$. They arrive at this number by reviewing the distribution of the oxygen abundances of star forming dwarf galaxies in the literature. The distribution peaks at $1 / 10 Z_{\odot}$ and drops sharply toward lower 
metallicities. In addition they point out that across a diverse body of stellar models the dependence on metallicity becomes critical at this level.

The metallicity of the Sun is a difficult question in of itself. For one the metallicity can be defined as the abundance of any metal. The abundance of a given metal is generally expressed as the logarithm of ratio of the number of the metal atoms to the number of hydrogen atoms. The two most common metals used to express the metallicity are iron and oxygen. Different values for the metallicity of an object relative to the Sun can found depending on the type of metal being measured. The fact that an object is deficient in one metal relative to the Sun does not necessarily mean it will be deficient in another. For instance iron is largely produced by Type Ia supernovae which do not occur until $\sim 10^{9}$ years after the onset of star formation. However Type II supernovae will begin enriching the ISM with oxygen after only $10^{7}$ years. The metallicity of XMPGs is usually given by the oxygen abundance for several reasons. Oxygen is the most abundant element in the Universe which was not formed during Big Bang nucleosynthesis. The oxygen abundance is more sensitive to the present enrichment of the ISM because it is returned to the ISM in large amounts more quickly than iron. As a practical matter the oxygen abundance of XMPGs can be measured more directly from strong nebular emission lines whereas iron emission and absorption lines are far more difficult to measure in these galaxies.

From the early 1980 s until recently the oxygen abundance of the Sun was widely agreed to be $12+\log (\mathrm{O} / \mathrm{H}) \sim 8.9$. Lambert $(1978)$ measured the value to be 8.92 from [O I] lines at optical wavelengths in the solar spectrum. Through measurement of the rotation lines of the $\mathrm{OH}$ molecule at 10.5 to 13 microns in the infrared Sauval, et al. (1984) 
deduced an abundance of 8.91 in perfect agreement with the optical lines. Grevesse, et al. (1984) found a similar value of 8.93 through analysis of $\mathrm{OH}$ vibration-rotation lines at 3 microns. However several observations in the past few years have lowered this value. Asplund, et al. (2004) use time-dependent, three dimensional models with departures from local thermodynamic equilibrium to fit forbidden $[\mathrm{O}$ I] lines, permitted $\mathrm{O} I$ lines, molecular $\mathrm{OH}$ vibration-rotation and pure rotation $\mathrm{OH}$ lines in the solar spectrum. They arrive at a value of $12+\log (\mathrm{O} / \mathrm{H})=8.66 \pm 0.05$. Meléndez (2004) measures the oxygen abundance from weak first overtone vibration-rotation $\mathrm{OH}$ lines in the $H$ band. This requires a solar spectrum with $\mathrm{S} / \mathrm{N}>10^{3}$ and a spectral resolution of $R=700000$. The abundance from this method is $\sim 8.6$.

In this thesis the solar oxygen abundance is assumed to be $12+\log (\mathrm{O} / \mathrm{H})=8.65$. As such an XMPG is defined as a galaxy for which $12+\log (\mathrm{O} / \mathrm{H})<7.65$.

It is also useful to have some benchmarks for galaxy metallicities. Esteban, et al. (2005) measure the oxygen abundances of eight $\mathrm{H}$ II regions in the Milky Way which range from $12+\log (\mathrm{O} / \mathrm{H})=8.57$ to 8.80 . They also measure a radial metallicity gradient in the galaxy with more metal rich regions near the galactic center. Skillman, Kennicutt, \& Hodge (1989) gives 8.34 as the oxygen abundance of the Large Magellanic Cloud and 7.98 for the Small Magellanic Cloud. In general metal content increases with the mass of a galaxy. Tremonti, et al. (2004) measure the mass-metallicity relationship for SDSS galaxies. They find that the median oxygen abundance is 8.63 for $10^{9} M_{\odot}$ galiaxics, 8.94 for $10^{10} M_{())}$, and 9.11 for $10^{11} M_{\odot}$. One explanation for this relationship is that low mass galaxies have smaller gravitational potentials which result in metals being expelled by galactic winds more 
so than in massive galaxies with deeper potential wells (Larson 1974). Given that mass and luminosity are correlated to first order there is a similar relationship between metallicity and luminosity such that the most luminous galaxies also tend to be the most metal rich.

As the above relationships suggest XMPGs are expected to be dwarf galaxies with low masses and luminosities. Most of the known and well-studied XMPGs are blue compact dwarts (BCDs). These are galaxies undergoing a significant episode of star formation. This fact gives them distinctive blue colors and high surface brightnesses. In addition they can be identified by strong nebular emission lines. Even so they are still faint which limits the distance to which they can be detected. The term "compact" was first used by Zwicky (1965) because they can be mistaken for point sources in ground surveys.

BCDs are not the only type of XMPGs. There are also dwarf ellipticals, dwarf spheroidals, dwarf irregulars, and low surface brightness galaxies with low metallicities. These classes of galaxies converge at low luminosities. However Chapter 6 , as well as most studies of XMPGs, focuses on BCDs. This is due to the fact that they are much easier to detect. While BCGs corner the conversation it should be kept in mind that in reality the pantheon on XMPGs may not be dominated by BCDs.

\subsubsection{Zwicky 18}

No discussion of XMPGs is complete without the mention of I Zw 18. I $\mathrm{Zw} 18$ is by far the most studied XMPG and was for many yoars the lowest metallicity galixy known. It was first described by Zwicky (1966) as a double system of compact galaxies. A modern HST image of $\mathrm{I} \mathrm{Zw} 18$ is shown in Figure 1.5. It was later found that it is actually two bright areas of star formation separated by $5.8^{\prime \prime}$. The brighter region is referred to as 
the NW region, which is the upper and rightmost of the pair in the figure, and the fainter region is referred to as the SW region. CCD imaging by Davidson, Kimman, \& Friedman (1989) revealed a more complex structure in the optical. They point out a component C, which was earlier sketched by Zwicky (1966), as a diffuse region 22" to the northwest of the NW component. This is the prominent object in the upper right hand corner of Figure 1.5.

The special nature of I Zw 18 began to be appreciated by Sargent \& Searle (1970). Their spectroscopy revealed strong star formation was taking place but there was lar less flux in the red than expected. This led them to the tentative conclusion that low mass star formation was supressed. The abundance analysis of Searle \& Sargent (1972) was the first to reveal the extremely low metal content of I $\mathrm{Zw} 18$. They were also the first to speculate that I Zw 18 may be extremely young. The SDSS spectra of I Zw 18 is shown in Figure 1.6. It is marked by very strong emission lines, indicative of active star formation. However the continuum is very weak and exceptionally blue, suggesting a small population of older stars.

More recent analyses of the abundances in $\mathrm{I} Z \mathrm{Zw} 18$ are in general agreement. Dufour, Garnett \& Shields (1988) measure an oxygen abundance of $12+\log (\mathrm{O} / \mathrm{H})=7.24$ in the NW region which is the most metal poor component. Thuan \& Izotov (2005) find abundances of $7.17 \pm 0.01$ and $7.22 \pm 0.01$ in the NW and SE components. This abundance is $\sim 1 / 50$ of the solar value.

In addition to the abundances there are other observations which point to the youth of I $\mathrm{Zw}$ 18. Izotov \& Thuan (2004) used deep HST ACS images of I Zw 18 to measure its resolved stellar population. They find no red giant branch which indicates the 
stellar population must be younger than $500 \mathrm{Myr}$. They also find evidence of three star formation episodes separated by $\sim 100 \mathrm{Myr}$. Near infrared imaging of I $\mathrm{Zw} 18$ by Hunt, Thuan, \& Izotov (2003) puts an upper limit of $22 \%$ of the mass being contributed by stars older than $500 \mathrm{Myr}$.

\subsubsection{Properties of Other Known XMPGs}

The record for the most metal poor galaxy known was recently broken by SBS 0335-052W. Izotov, Thuan, \& Guseva (2005) measure the oxygen abundance to be $12+$ $\log (\mathrm{O} / \mathrm{H})=7.12 \pm 0.03$ which is slightly lower than the $7.17 \pm 0.01$ measured for the NW component of I Zw 18. SBS 0335-052W is a companion of the BCD SBS 0335-052E, another XMPG with an oxygen abundance of $\sim 7.30$ (Izotov, et al. 1990). The nature of SBS 0335052W lay below the radar due to the fact that it is considerably fainter than SBS 0335-052E. Like I Zw 18 the age of the oldest stars is determined to be at most $500 \mathrm{Myr}$ (Izotov, Thuan, \& Guseva 2005).

Kniazev, et al. (2004) discovered eight new XMPGs from the SDSS spectroscopy. At the time this accounted for a quarter of all XMPGs known as they are difficult to find. One of these, SDSS J0519+0007, was the most distant (177 Mpc) and most luminous $\left(M_{g} \sim-18.6\right)$ XMPG known. The luminosities of the sample covered a surprisingly large range, $-12.4<M_{g}<-18.6$.

Izotov, et al. (2006) report the discovery of two XMPGs selected from the SDSS which are among the five most metal poor galaxies known. The oxygen abundances of SDSS J2104-0035 and SDSS J0113+0052 are $7.26 \pm 0.03$ and $7.17 \pm 0.09$ respectively. 


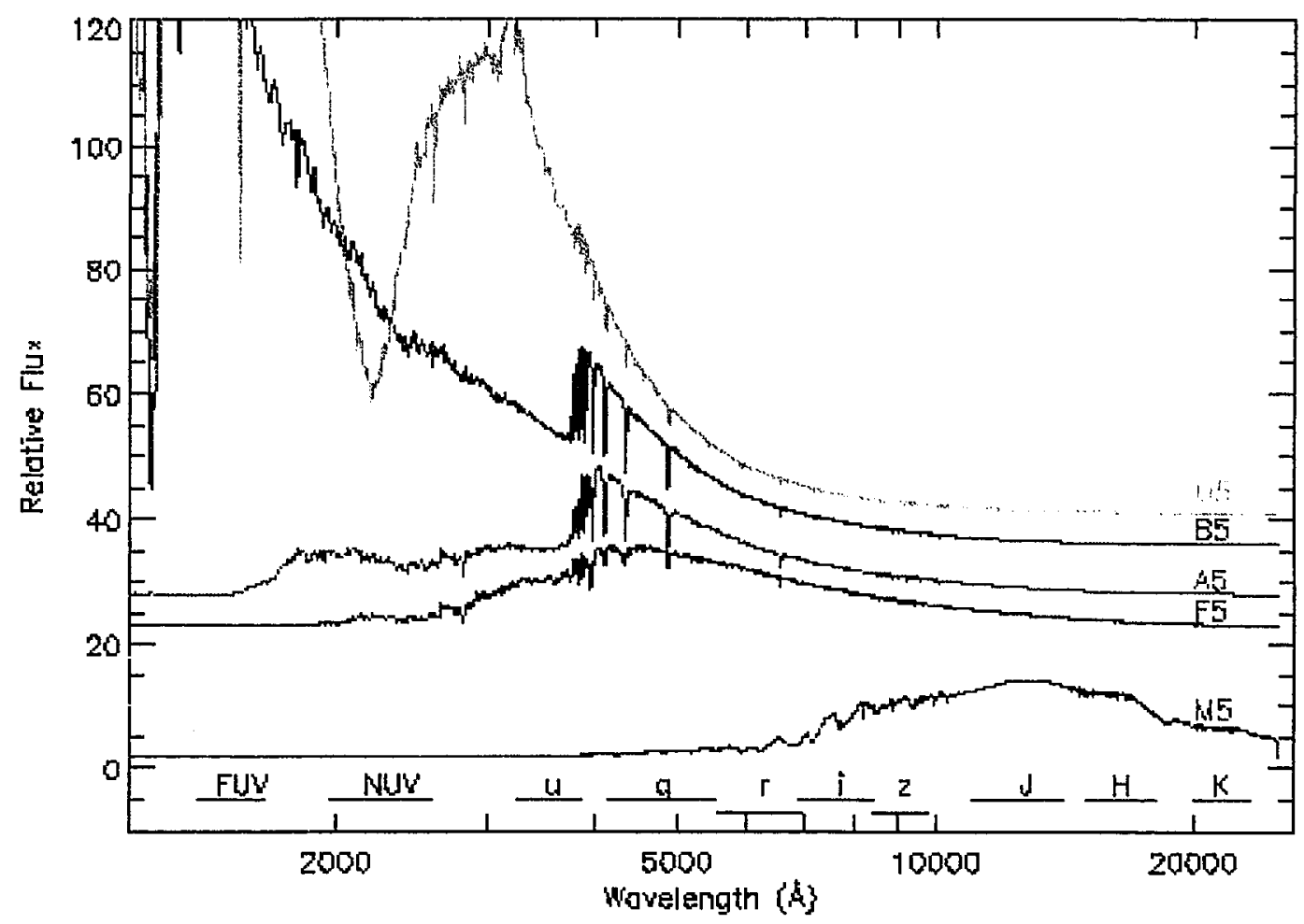

Figure 1.1 Empirical spectra from the Pickles (1998) database for the main sequence stars in Table 1.1. The spectra are scaled to show their relative shapes. O5 stars are actually $10^{8}$ times more luminous than M5 stars. Along the bottom are the FWHM of the photometric filters that will be used in this project centered on the effective wavelengths of the respective filters, to give an idea what spectral features lie in each bandpass. 

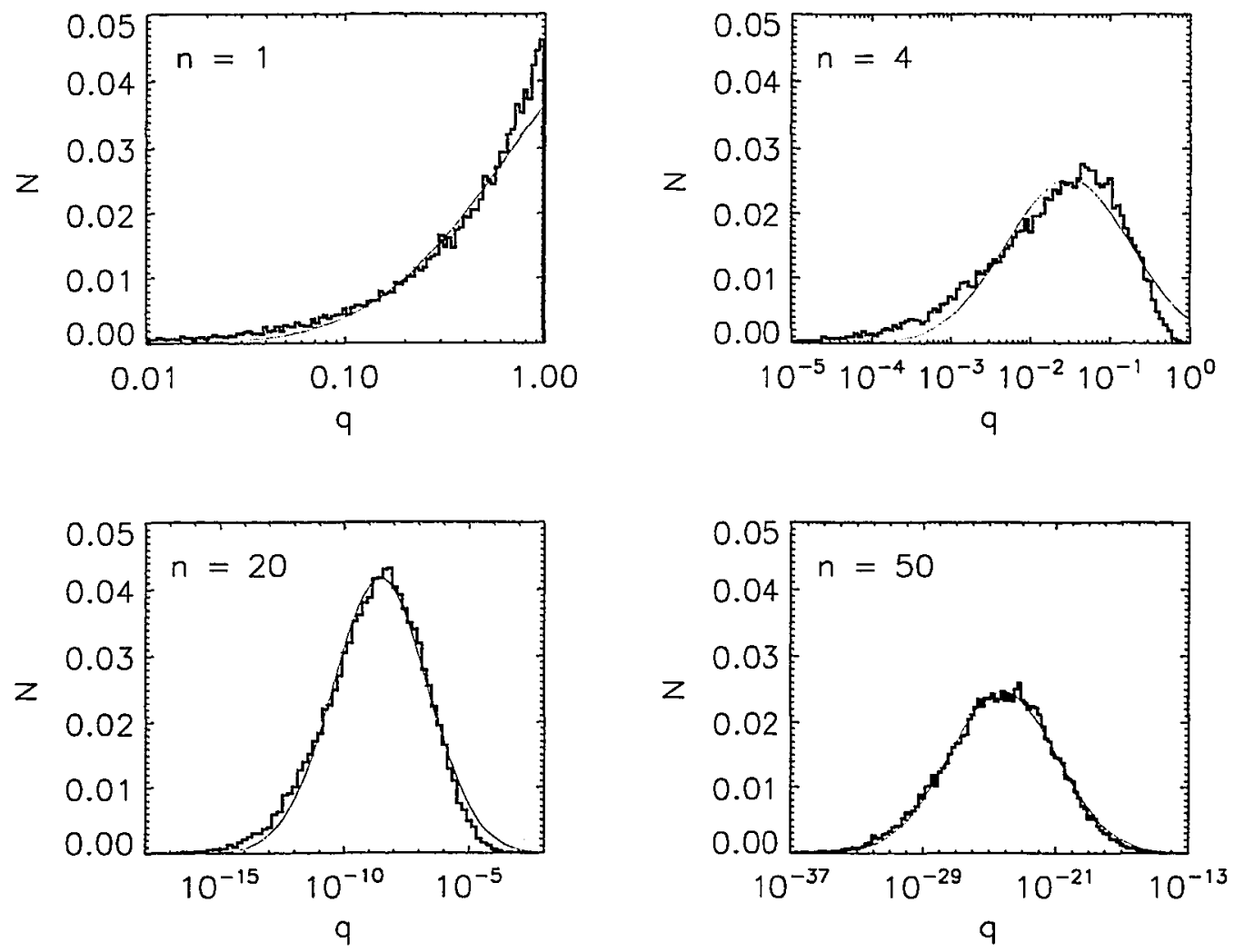

Figure 1.2 Distributions of $q=x_{1} x_{2} \ldots x_{n}$ for $n=1,4,20,50$. The variables $x_{i}$ are random numbers uniformly distributed between 0 and 1 . For each plot $N=30000$. The red line is the best fitting normal distribution of $\log q$. 


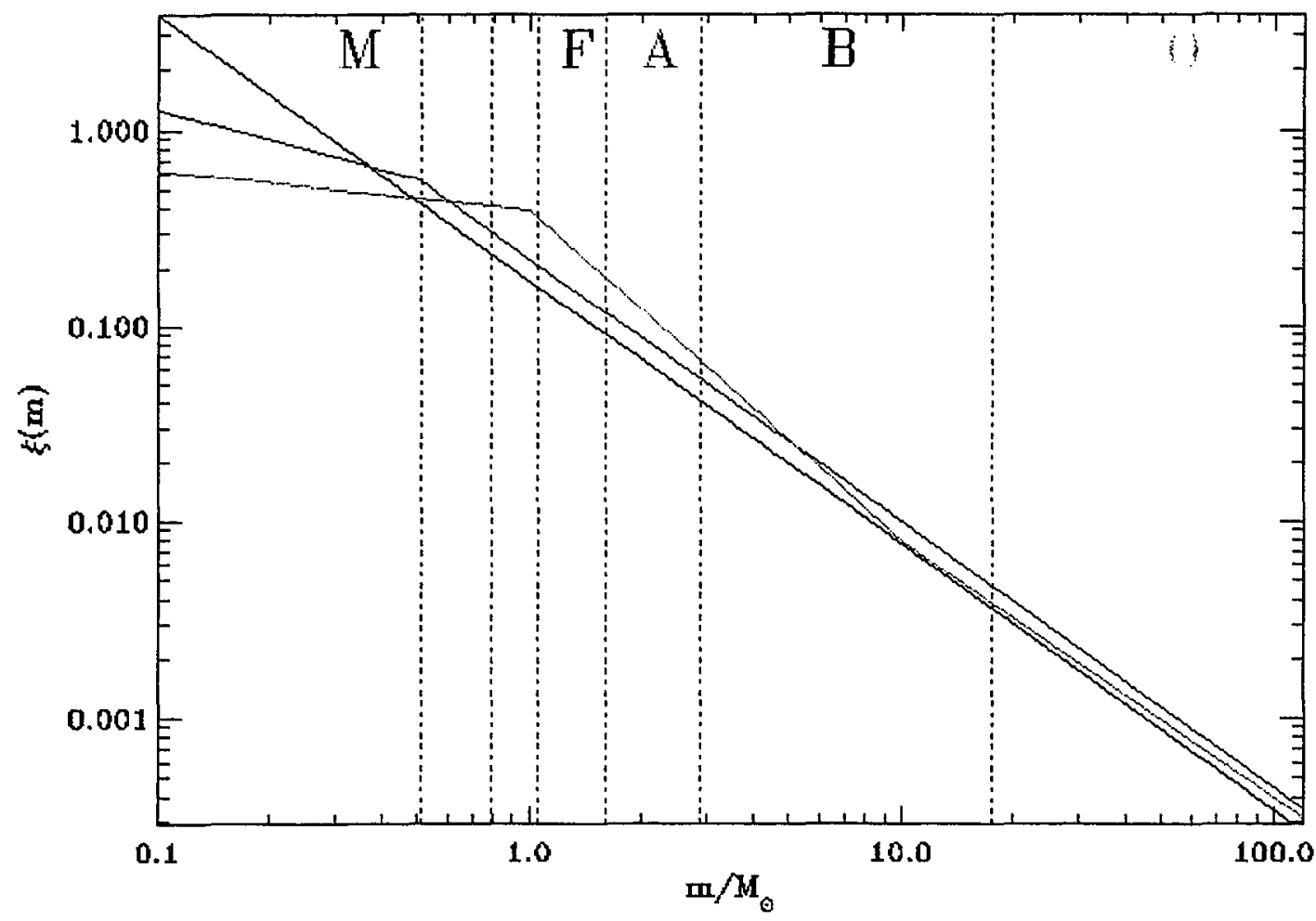

Figure 1.3 Selected stellar IMFs. The black curve is Salpeter's original IMF. The red curve is Scalo's recommended three part IMF from 1998. The blue curve is the $\Gamma$ parameterization of Salpeter's IMF used in our models. The dotted lines indicate the mass boundaries between main sequence spectral types. 


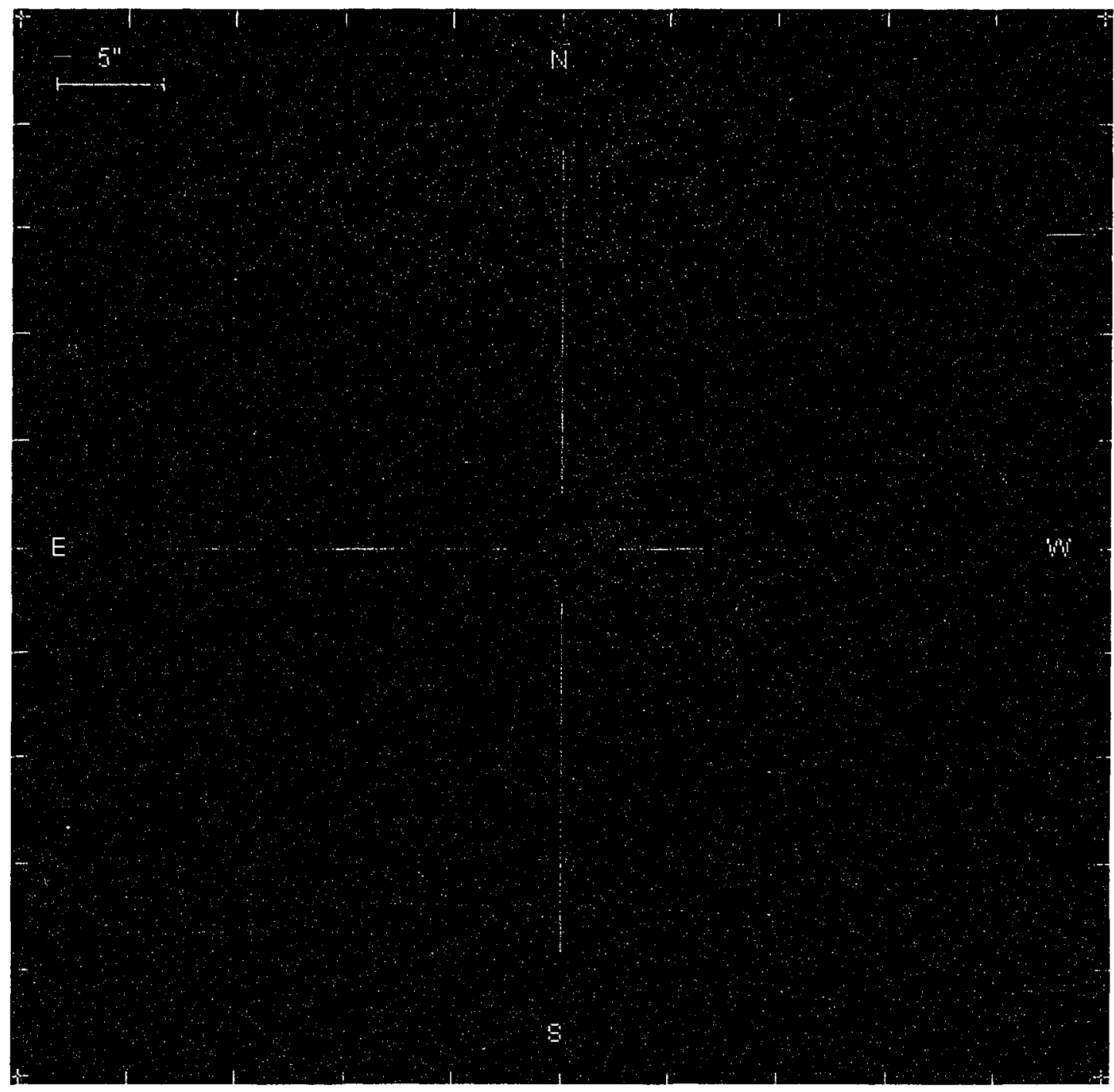

Figure 1.4 SDSS image of the graviational lens SDSS $11004+1112$. Fom images of the same $z=1.734$ quasar are clearly visible around the central cluster galaxy at $z=0.68$. The maximum separation of the images is $14.6 "$, double the largest previously known separation. 


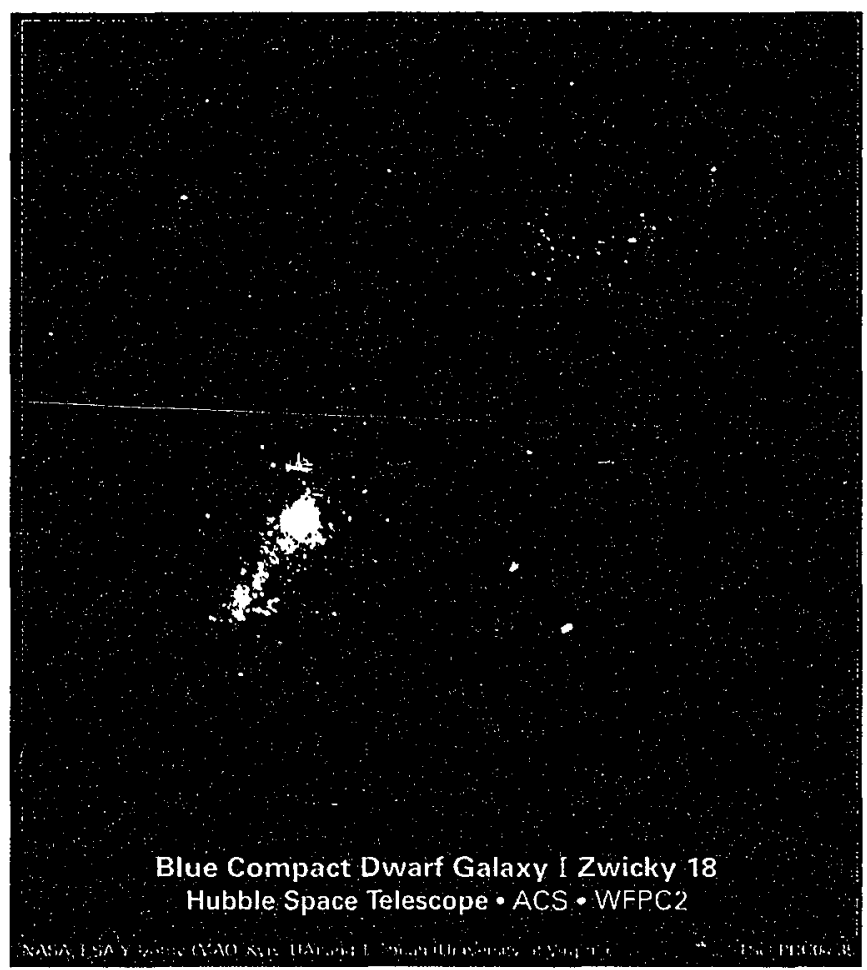

Figure 1.5 Hubble Space Telescope image of the extremely low metallicity blue compact dwarf galaxy I Zw 18. The image was taken with the ACS and WFPC2 cameras. I Zw 18 is the bright blue object at lower left. The northwet component, thebright part on the upper right, of I $\mathrm{Zw} 18$ is the area with the lowest metallicity. Image courtesy of the Space Telescope Science Institute. 
$R A=143.50845, D E C=55.24107, M J D=52266$, Plate $=555$, Fiber $=558$

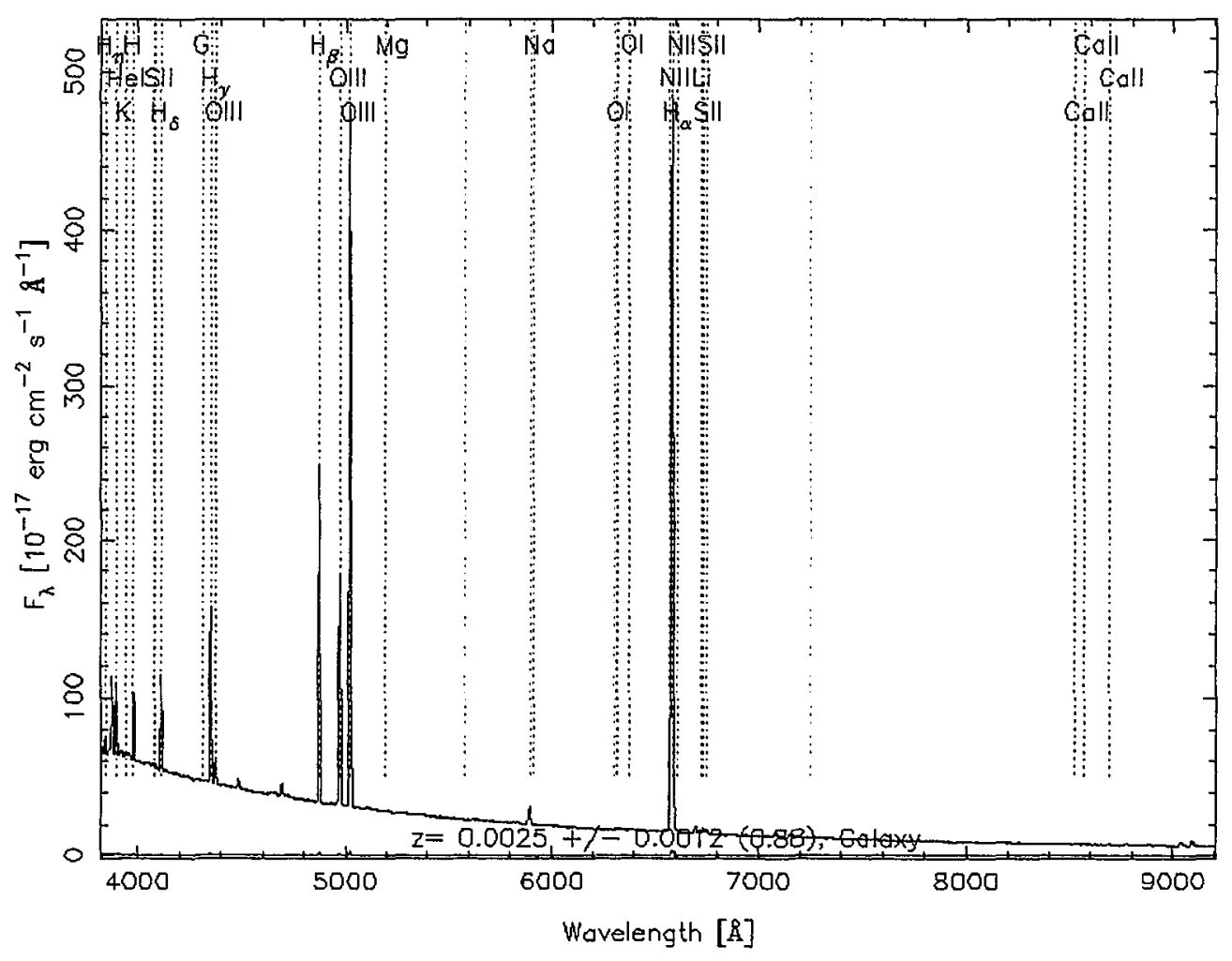

Figure 1.6 SDSS spectrum I Zw 18, of the most metal poor galaxies known. Vertical dotted lines mark the positions of selected absorption and emission lines at the measured redshift of $z=0.0025$. The green line shows the error in the spectrum as a function of wavelength. 


\section{Chapter 2}

\section{The Stellar Initial Mass Function: Sample Selection and Preliminaries}

\subsection{Methodology}

This chapter revisits the "classic" method of K83 (and the subsequent extension KTC94) to constrain the IMF of integrated stellar populations. The method takes advantage of the sensitivity of the $\mathrm{H} \alpha$ equivalent width (EW) to the IMF. K83 showed that model IMF tracks can be differentiated in the $(B-V)-\log (\mathrm{H} \alpha \mathrm{EW})$ plane.

The total flux of a galaxy at $6565 \AA$ is the combination of the underlying continuum flux plus the flux contained in the Ho emission line. The H $\alpha$ flux and the continuum flux have different physical origins, both of which can be used to gain physical insights into galaxies.

In the absence of AGN activity the $\mathrm{H} \alpha$ flux is predominantly caused by massive $O$ and $B$ stars which emit ionizing photons in the ultraviolet. $O$ and $B$ stars are young and 
found in the regions of ionized hydrogen in which they formed. In Case B recombination, where it is assumed that these clouds are optically thick, any emitted Lyman photons are immediately reabsorbed. After several scattering events the Lyman photons are converted into lower series photons (including $\mathrm{H} \alpha$ ) and two photon emission in the $2^{2} S \rightarrow 1^{2} S$ contimuum. These photons experience smaller optical depths and can escape the cloud. The transition probabilities are weakly dependent on electron density and temperature and can be calculated. Through this process the measured $\mathrm{H} \alpha$ flux can be converted into the number of $\mathrm{O}$ and $\mathrm{B}$ stars currently burning in an integrated stellar population.

However Case B recombination is an idealized condition and it is possible that ionizing photons can escape the cloud without this processing; a situation known as Lyman leakage. As such the $\mathrm{H} \alpha$ flux is a lower limit on the number of $\mathrm{O}$ and $\mathrm{B}$ stars present.

The continuum flux of a galaxy is due to the underlying stellar population. At $6565 \AA$ the continuum is dominated by red giant stars in the $0.7-3 \mathrm{M}_{\odot}$ range while the $\mathrm{H} \alpha$ flux comes from stars more massive than $10 \mathrm{M}_{\odot}$.

The EW is defined as the width in angstroms of an imaginary box with a height equal to the continuum flux level surrounding an emission or absorption line which contains an area equal to the area contained in the line. This is effectively the ratio of the strength of a emission or absorption line to the strength of the continuum at the same wavelength. Given the physical origins of the $\mathrm{H} \alpha$ flux and the continum at $6565 \AA$ the $\mathrm{H} \alpha \mathrm{EW}$ is the ratio of massive $O$ and $B$ stars to stars around a solar miss. Therefore the Ifex EW is sensitive to the IMF slope above around $1 \mathrm{M} \odot$ and can be used to probe the IMF in galaxies. 
As mentioned in the introduction several degeneracies plague the study of the IMF from the integrated light properties of galaxies. Variations in the IMF, age, metallicity, and SFH of galaxy models can all yield similar effects in the resulting spectra. For example, increasing the fraction of massive stars, reducing the age of a galaxy, lowering the metallicity, and a recent increase in the star formation rate will all make a galaxy bluer.

Metallicity effects were not discussed in either K83 or KTC94 and galaxy ages were assumed, $15 \mathrm{Gyr}$ for K83 and $10 \mathrm{Gyr}$ for KTC94.

The SFH in K83 is addressed by calculating models with exponentially decreasing SFHs for a range of e-folding times, as well as a constant and a linearly increasing SFH. In the $(B-V)-\log (\mathrm{H} \alpha \mathrm{EW})$ plane the effect of varying the $\mathrm{SFH}$ e-folding time is orthogonal to IMF variations. However this is only true for smoothly varying exponential and linear SFHs. Discontinuities, either increases (bursts) or decreases (gasps), in the star formation rate can affect the $\mathrm{H} \alpha \mathrm{EW}$ relative to the color in ways similar to a change in the IMF. Along with the exponential SFHs KTC94 also uses models with instantaneous bursts on top of constant SFHs. However this was done to access high EWs at an age of 10 Gyr rather than to fully flesh out the effects of SFH discontinuities.

The assumption of smoothly varying SFHs is a key assumption in our analysis. Most late-type galaxies are thought to form stars at a fairly steady rate over much of recent time although bursts of star formation may play a significant role in low mass galaxies (Kennicutt 1998b). For these galaxies smoothly varying SFIIs are justified. However there are other galaxies clearly in the midst of a strong burst of star formation (e.g. M82) and dwarf galaxies with complex SFHs, e.g. NGC 1569 (Angeretti, et al. 2005), for which this 
assumption is a poor one. The effects of violations of our SFH assumptions are described in detail in the results section.

\subsection{The Data}

K83 cites four major sources of error all of which are improved upon or eliminated by the high, uniform quality of SDSS spectroscopic and photometric data.

The $\mathrm{H} \alpha$ fluxes in his sample can be contaminated by nonthermal nuclear emission. In the updated investigation, KTC94, this problem is addressed by removing objects with known Seyfert or LINER activity and luminous AGN. The SDSS spectra allow measurements of emission line ratios which can be used to separate star forming galaxies from AGN (Baldwin, Phillips, \& Terlevich 1981).

The second problem is that the $\mathrm{H} \alpha$ emission flux will be underestimated if the underlying stellar absorption of $\mathrm{H} \alpha$ is not taken into consideration. The narrow band filter photometry of Kennicutt \& Kent (1983) could not measure this effect for individual galaxies so a fixed ratio was assumed for all galaxies. The SDSS spectroscopic pipeline does not take this into account either. We use the H $\alpha$ fluxes measured from the SDSS spectra by Tremonti, et al. (2004) which fit the continua with stellar population models to more accurately measure the $\mathrm{H} \alpha$ emission. While the SDSS pipeline method is sufficient for strong emission lines the $\mathrm{H} \alpha$ absorption EW can be as large as $5 \AA$ which is significant for weaker emission lines.

Thirdly, their narrow band $\mathrm{H} \alpha$ imaging includes [N II] emission which is corrected for by assuming a constant $[\mathrm{N} \mathrm{II}] / \mathrm{H} \alpha$ ratio. The $\mathrm{H} \alpha$ and $[\mathrm{N}$ II] emission lines are resolved 
in the SDSS spectra so there is no need for a correction. This is a significant improvement. Kennicutt \& Kent (1983) found from a literature survey that the mean value of the $\mathrm{H} \alpha /(\mathrm{H} \alpha$ $+[\mathrm{N} \mathrm{II}])$ ratio is $0.75 \pm 0.12$ for spiral galaxies and $0.93 \pm 0.05$ in irregular galaxies. These mean corrections were applied uniformly to the K83 data. In KTC94 a uniform correction was applied using $[\mathrm{N} \mathrm{IT}] / \mathrm{H} \alpha=0.5$. For comparison in our sample the mean value of the $\mathrm{H} \alpha /(\mathrm{H} \alpha+[\mathrm{N} \mathrm{II}])$ ratio is a strikingly similar 0.752 and the mean $[\mathrm{N} \mathrm{II}] / \mathrm{H} \alpha$ ratio is 0.340. However [ $\mathrm{N} \mathrm{II}] / \mathrm{H} \alpha$ ranges from 0.0 to 0.6 . If our $\mathrm{H} \alpha$ and $[\mathrm{N} \mathrm{II}]$ lines were blended, applying a fixed correction would introduce errors of as much as $25 \%$ in the $\mathrm{H} \alpha \mathrm{EWs}$ of individual galaxies.

Lastly, in both K83 and KTC94 extinction corrections were addressed by plotting data alongside models which were either assumed an average value for the extinction or were unextincted. The Balmer decrement $(\mathrm{H} \alpha / \mathrm{H} \beta)$ can be measured from SDSS spectra. which allows for extinction corrections for individual galaxies.

Another major advantage of this study is that the sample size is much larger than that of K83 and KTC94. The KTC94 sample contains 210 galaxies, whereas ours has $\sim 10^{5}$. The large sample size allows us to investigate IMF trends as functions of galaxy luminosity, redshift and aperture fraction with subsamples larger than the entire KTC94 sample.

There is a key disadvantage to this method as well. K83 and KTC94 were able to adjust the sizes of their photometric apertures to contain the entire disk of individual galaxies to a liniting isophote of $25 \mu$ given by the RC2 caltalog (Kennicuth \& Kent, 1983). The advantage of narrow band measurements of $\mathrm{H} \alpha \mathrm{EW}$ is that they can cover a much larger aperture and match the broadband measurements set to match the physical size of 
individual galaxies. The SDSS has fixed 3" spectroscopic apertures. This problem is partly offset by using matching 3" photometry apertures from the SDSS. However this introduces aperture effects as observed galaxies have a wide range of angular sizes due to the range of physical sizes and distances present in the local universe. This is significant as radial metallicity gradients (e.g. Vila-Costas \& Edmunds (1992)) are observed in spiral galaxies which could incorrectly be interpreted as radial IMF gradients.

Even so, this method can constrain the IMF within the SDSS apertures. For our program galaxies $23 \%$ of the total light falls in the SDSS apertures. The fact that the more distant galaxies are more luminous and tend to be larger helps to balance out the larger physical scales of the fixed aperture size at greater distances. On average $17 \%$ of the light falls in the aperture for the faintest galaxies while it is $25 \%$ for the brightest bin. In spite of their limited size the SDSS apertures still contain a great diversity of stellar populations which make this data set an excellent test bed for IMF universality. We will present extensive tests of aperture effects below.

\subsubsection{Sample Selection}

The sample is selected from Sloan Digital Sky Survey data. The project goal of the SDSS is to image a quarter of the sky in five optical bands with a dedicated $2.5 \mathrm{~m}$ telescope (York, et al. 2000). From the imaging $10^{6}$ galaxies and $10^{5}$ quasars will be selected for spectroscopic followup. Photometry is done in the ugriz filter system described by Fukugitan, et al. (1996). Magnitudes are on the arcsinh system (Lupton, Gumn \& Szalay 1999) which approaches the AB system with increasing brightness. Spectra are taken with a multi-object

fiber spectrograph with wavelength coverage from $3800 \AA$ to $9200 \AA$ and $R \sim 1800$ (Uomoto, 
et al. 1999).

Our sample is a sub-sample of the Main Galaxy Sample from SDSS DR4 (Adelman1McCarthy et al. 2006). The Main Galaxy Sample (MGS) targets galaxies with $r \leq 17.77$ in Petrosian magnitudes (Stoughton, et al. 2002). All galaxies in the MGS are strong detections so the differences between luptitudes and the AB system can be ignored. In order to avoid fiber crosstalk in the camera an upper brightness limit of $g=15.0, r=15.0$ and $i=14.5$ is imposed. Targets are selected as galaxies from the imaging by comparing their PSF magnitudes to their de Vaccouleur's and exponential profile magnitudes. Exposure times for spectroscopy are set so that the cumulative median signal-to-noise satisfies $(\mathrm{S} / \mathrm{N})^{2}>15$ at $g=20.2$ and $i=19.9$ fiber magnitudes. The time to achieve this depends on observing conditions but always involves at minimum three 15 minute exposures.

Due to the construction of the spectrograph fibers cannot be placed closer than 55 " to each other. This may be a source of bias in the sample. Cluster galaxies may be preferentially excluded from the sample. The SDSS collaboration has plans to quantify this effect in the near future (Adelman-McCarthy et al. 2006). LSBs are excluded from the MGS with a surface brightness cut which may also bias the sample (Stoughton, et al. 2002). There is some evidence that LSBs may have IMFs which differ from a universal IMF. Lee, et al. (2004) find that the comparatively high mass-to-light ratios of LSBs can be explained with an IMF deficient in massive stars relative to normal galaxies.

Our sample begins with the fourth data release (DR4) of the SDSS (AdelmanMcCarthy et al. 2006). DR4 covers $4783 \mathrm{deg}^{2}$ in spectroscopy for a total of 673,280 spectra, 567,486 of which are galaxy spectra. 429,748 of these have flags set indicating they are 
part of the MGS. DR4 also includes special spectroscopic observations of the Southern Stripe which were not selected by the standard algorithm but which nonetheless have the TARGET_GALAXY flag set in primTarget which usually indicates membership in the MGS. These objects are identified by comparing their spectroscopic plate number to the list of special plates in (Adelman-McCarthy et al. 2006) and rejected. This leaves 423,285 spectra.

The first round of cuts to our sample address general data quality. While the overall quality of SDSS data is high there are a handful of objects with pathological values for one or more parameters. First we require that all parameters of interest have reasonable, real values. This means that the Petrosian and fiber magnitudes must be between 0 and 25 and have errors smaller than 0.5 in all five ugriz bands. Line flux errors are capped at $10^{-12} \mathrm{erg} \mathrm{cm}^{-2} \mathrm{~s}^{-1} \AA^{-1}$ and equivalent widths at $10^{5} \AA$. For most of the parameters less than $1 \%$ of objects fail this loose requirement. However $6.6 \%$ of the objects fail the Petrosian magnitude error requirement. This is most likely due to the photometric pipeline having a difficult time defining the Petrosian radius. As such this constraint is potentially biased against LSBs or galaxies with unusual morphologies. In defense of this cut we later bin our data by luminosity and aperture fraction both of which are determined in part by the Petrosian magnitudes and also by $K$-corrections determined from them. In addition, limiting flux errors to $50 \%$ is hardly unreasonable. Altogether 391,160 galaxies pass these combined requirements, which is $92.4 \%$ of the MGS.

Galaxics from plodometry Run 1659 are removed because of a known problem with the photometry. This excludes 4,485 galaxies (1.1\%) from a continuous strip on the sky and should not be a source of bias. We place a further constraint on the $z$ band fiber 
magnitude requiring that the error be less than 0.15 . The $z$ band generally suffers from the most noise so this requirement ensures that the fiber magnitude quality is good enough to minimize the chance of erroneous $K$-corrections which could affect our colors. Only 2,866 (0.7\%) MGS galaxies fail this test.

Combining the general data quality requirements, the Run 1659 rejection and the fiber $z$ band error limit leaves 386,647 galaxies (91.3\% of the MGS).

The next round of cuts to our sample, while necessary, have clear astrophysical implications. Many of the objects in the MGS have AGN components. As we are interested in studying only the underlying stellar populations of these objects AGN must be removed. This is done using the classical Baldwin, Phillips, \& Terlevich (1981) diagram comparing the logarithms of the [O III $\lambda 5007] / \mathrm{H} \beta$ and [N II $\lambda 6584] / \mathrm{H} \alpha$ emission line ratios. We used the criterion of Kauffmann, et al. (2003) where objects for which

$$
\log ([\mathrm{O} \mathrm{III}] / \mathrm{H} \beta)>\frac{0.61}{\log ([\mathrm{N} \mathrm{II}] / \mathrm{H} \alpha)-0.05}+1.3
$$

are classified as AGN and rejected. Following Brinchmann, et al. (2004) we require the S/N of the $\mathrm{H} \alpha, \mathrm{H} \beta,[\mathrm{O}$ III] and [N II] lines to be at least 3 to properly classify a galaxy as a star forming one. 131,807 galaxies (34.1\% of MGS objects surviving our first round of cuts) survive this cut.

The above cut automatically rejects any galaxies with weak [O III] and [N II] lines. This excludes galaxies with weak star formation. To a lesser extent metal poor galaxies which also have weak [N II] emission are rejected as well. Brinchmann, et al. (2004) also define a low $\mathrm{S} / \mathrm{N}$ star forming class of galaxies which we identify and keep in our sample. These are the galaxies which have not already been classified as star forming or AGN by 
strong lines and equation 2.1, nor have been identified as low S/N AGN by [N II $\lambda 6584] / H \alpha$ $>0.6$ with $\mathrm{S} / \mathrm{N}>3$ in both lines, yet still have $\mathrm{H} \alpha$ with $\mathrm{S} / \mathrm{N}$ at least $2.79,548(20.6 \%)$ of the sample falls into the low $\mathrm{S} / \mathrm{N}$ star forming galaxy category. Combining the two classes $211,355(54.7 \%)$ of the galaxies survive the AGN cut.

The AGN cut also has a strong luminosity bias for two reasons. Galaxies with AGN components tend to be brighter. The bimodal distribution of galaxies in color-magnitude space (Baldry, et al. 2004) also plays a role. The most Iuminous galaxies are predominantly red with minimal star formation and thus weak emission lines. Luminous galaxies are rejected both for having $A G N$ components and for having low $\mathrm{S} / \mathrm{N}$ emission lines. Over $95 \%$ of galaxies fainter than $\mathrm{M}_{r, 0.1}=-19$ meet this criteria, but by $\mathrm{M}_{r, 0.1}=-24$ the fraction is only $38.9 \%$.

A color bias is also introduced by the AGN cut. Over $95 \%$ of galaxies bluer than $(g-r)_{0.1}=0.6$ pass, which drops to $24 \%$ by $(g-r)_{0.1}=1.2$. This is mainly due to the $\mathrm{S} / \mathrm{N}$ requirement for the emission lines. Redder galaxies tend to have weak emission lines and are rejected.

The Balmer decrement is used for the extinction correction so $\mathrm{H} \alpha$ and $\mathrm{H} \beta \mathrm{S} / \mathrm{N}$ are required to be at least 5 to reduce errors. 214,912 galaxies (55.6\%) have $\mathrm{H} \beta \mathrm{S} / \mathrm{N}>5$ which is the more restrictive of the two criteria. This cut has a clear luminosity bias. Roughly $85 \%$ of galaxies with $M_{r, 0.1}>-20$ satisfy this requirement, but this fraction decreases with increasing lunninosity until only $12.9 \%$ survive at $\mathrm{M}_{r, 0.1}=-24$. This is again due to the bimodal distribution of galaxies. The luminous red galaxies with weak emission lines are rejected. 
There is also a color bias. Over $93 \%$ of the bluest galaxies blueward of $(g-r)_{0.1}=$ 0.8 survive the cut while only $23 \%$ of the reddest pass this requirement. As previously mentioned, by nature the reddest galaxies have weak Balmer lines as a result, of their low SFRs and are preferentially rejected.

A redshift cut of $0.005 \leq z \leq 0.25$ is applied to ensure that peculiar velocities do not dominate at low redshift and to limit the range of galaxy ages. 384,349 galaxies (99.4\%) meet this criteria. Nearly all galaxies from $\mathrm{M}_{r, 0.1}=-17$ to -23 survive this cut. On the low luminosity end only $27.4 \%$ of $\mathrm{M}_{r, 0.1}=-14$ galaxies are distant enough to pass and on the high end $93.1 \%$ of $\mathrm{M}_{r, 0.1}=-24$ galaxies are close enough to survive. Only $63 \%$ of the bluest galaxies pass. Many of the blue galaxies that fail are actually H II regions of Local Group galaxies which are treated as their own objects by the SDSS pipeline so removing them actually improves the integrity of our sample.

The stellar populations of galactic bulges can be significantly different from those in the spiral arms. The SDSS fibers have a fixed aperture of 3" so over the large range of luminosities and distances in the MGS aperture affects can become very important. To remove outliers we require that at least $10 \%$ of the light from a galaxy falls within the spectroscopic aperture. This is done by comparing the Petrosian magnitude to a fixed 3" aperture fiber magnitude. Both of these quantities are calculated for all objects in the SDSS by the photometric pipeline. 371,777 (96.2\%) galaxies survive this cut. This cut rejects proportionally more faint galaxies; $99.2 \%$ pass at $\mathrm{M}_{r, 0.1}=-24$ compared to $50.9 \%$ at $\mathrm{M}_{r, 0.1}=-14$. The aperture cut has low sensitivity to color.

The intersection of the AGN, Balmer line $\mathrm{S} / \mathrm{N}$, redshift and aperture fraction cuts 
leaves 140,598 galaxies- $36.4 \%$ of the high quality MGS data defined by our first round of cuts and $33.2 \%$ of the MGS as a whole.

At this point three final cuts are applied to the sample. One galaxy is removed for surviving all criteria, but having a negative $\mathrm{H} \alpha \mathrm{EW}$.

The extinction of individual galaxies is estimated using the Balmer decrement ( $\mathrm{H} \alpha / \mathrm{H} \beta$ emission flux ratio) for each galaxy. Given Case B recombination, a gas temperature of $10,000 \mathrm{~K}$ and density of $100 \mathrm{~cm}^{-3}$ the Balmer decrement is predicted to be 2.86 (Osterbrock 1989). This ratio is weakly dependent on nebular temperature and density. Osterbrock (1989) lists values down to 2.74 for Case B recombination in environments where both the temperature and electron density are high. $3.2 \%$ of the galaxies suffer from the problem that the Balmer decrement is less than 2.86 and $2.1 \%$ have a Balmer decrement below 2.74. 538 galaxies ( $0.4 \%$ ) have Balmer decrements more than $3 \sigma$ below 2.74, which is around 6 times more than expected. This is does not suggest a problem with the Case $\mathrm{B}$ assumption as the predicted Balmer decrements for Case A recombination are nearly identical in each temperature regime.

To understand the reason behind this problem around 100 of the offending spectra were inspected revealing a few different causes for the problem. Around 80 galaxies are at redshifts where the telluric $\mathrm{O}$ I $\lambda 5577$ line affects the measurement of $\mathrm{H} \beta$. Many of these galaxies have very strong emission lines with extremely weak stellar absorption. Using the SDSS pipeline values instead of the Tremonti, et al. (2004) values yields acceptable Balmer decrements. The rest are galaxies with low flux where the Balmer lines are in absorption. This shows there are a few cases where attempting to fit the underlying stellar absorption 
lines fails and this failure is not reflected in the error values. These galaxies are rejected without any apparent introduction of bias.

All of these cuts combined leave 140,060 galaxies, which is $33 \%$ of the MGS and $36 \%$ of the high quality MGS data. After removing duplicate observations there are 130,602 galaxies in our sample. Of these objects $1.7 \%$ overlap with the Luminous Red Galaxy Sample.

Overall the bulk of the galaxies are removed by AGN rejection and the $\mathrm{H} \beta \mathrm{S} / \mathrm{N}$ requirement, with the rest of the cuts having little effect. Both of these cuts are necessary. AGN must be removed to ensure that the $\mathrm{H} \alpha$ emission represents the underlying stellar population and not an accretion disk. Our method requires that the galaxies have measurable Balmer emission lines. This coupled with the need for accurate extinction corrections justifies the Balmer line $\mathrm{S} / \mathrm{N}$ requirement.

Our cuts bias our sample by preferentially excluding galaxies at both luminosity and both color extremes. The faintest galaxies are most affected by the redshift and aperture cuts while the luminous galaxies succumb to the $\mathrm{H} \beta \mathrm{S} / \mathrm{N}$ requirement. At the red extremes it is the $\mathrm{H} \beta \mathrm{S} / \mathrm{N}$ and $\mathrm{AGN}$ requirements that play equally large roles, while the bluest objects are primarily rejected by the Hubble flow redshift requirement. Although our sample is biased by our cuts each one is a necessary evil. We do not attempt to correct this bias, but we remind the reader that the following results are only representative of actively star forming galaxics without any AGN activity.

The aim of this paper, however, is to test IMF universality. If the IMF is truly universal it should be universal in any subsample of galaxies. The fact that our sample is 
slightly biased with respect to luminosity and color is not a significant barrier to achieving our goal.

\subsubsection{Corrections}

The SDSS includes a number of different calculated magnitudes. We use the fiber magnitudes which are 3" fixed aperture magnitudes. Although it was not the case in earlier versions of the photometric pipeline, fiber magnitudes are now seeing corrected (Abazajian, et al. 2004). The fiber magnitudes were not originally intended for science purposes but rather to get an idea of how bright an object will appear in the spectrograph. We use the fiber magnitudes to reduce the aperture effects arising from comparing a 3" spectroscopic aperture to Petrosian magnitudes. Originally the SDSS used "smear" exposures to correct spectra for light falling outside the 3" aperture due seeing, guiding errors and atmospheric refraction (Stoughton, et al. 2002). The smear technique was later found to be an improvement only for high S/N point sources and its use was discontinued (Abazajian, et al. 2004). The spectra here are not seeing corrected.

After paring the sample to its final size a number of corrections must be made to both the photometric and spectroscopic data. Galactic reddening from the Milky Way must be corrected for. SDSS database includes the Schlegel, Finkbeiner, \& Davis (1998) dust map values for each photometry object.

The extinction of individual galaxies is estimated using the Balmor decrement for each galaxy. The data is corrected assuming that 2.86 is the true value of the Balmer decrement using the Milky Way dust models of Pei (1992). The assumption of Milky Way dust is not significant as models of the dust attenuation in the Milky Way, SMC and 
LMC are nearly identical in the $g$ band and redward. As aforementioned a few percent of our galaxies have Balmer decrements below 2.86. Our solution is to set the emission line extinction to $A_{V, l}=0.01$ magnitudes for these galaxies.

Massive young stars and their surrounding ionized nebulae tend to be embedded in their star forming regions more so than older, lower mass stars which have had time to migrate from their birth regions. As such nebular emission lines will experience more extinction than the continuum. Calzetti, Kinney, \& Storchi-Bergmann (1994) find the ratio of emission to continuum line extinction is $f=2.0 \pm 0.4$. We assume this value to be 2.0 and correct the continuum and emission lines separately. This is the same value used by K83 and KTC94 in their extinction corrected models. We note that the spatial geometry of the dust can influence the extinction law, but this complication is beyond the scope of this paper.

Galaxy photometry is $K$-corrected to $z=0.10$ using version 4.1 .4 of the code of Blanton, et al. (2003a). This redshift is roughly the median of the sample and is selected to minimize errors introduced by the $K$-corrections. The $(g-r)_{0.1}$ colors we use are the $g-r$ colors we would observe if the galaxies were all located at $z=0.1$.

Stated explicitly the $(g-r)_{0.1}$ color is

$$
c=(g-r)_{0.1}=\left(g-k_{g}-\mathrm{A}_{g}-1.153 \mathrm{~A}_{\mathrm{V}, l} / f\right)-\left(r-k_{r}-\mathrm{A}_{r}-0.834 \mathrm{~A}_{\mathrm{V}, l} / f\right)
$$

where $k_{g}$ and $k_{r}$ are $K$-corrections, $\mathrm{A}_{g}$ and $\mathrm{A}_{r}$ are Milky Way reddening values, and 1.153 and 0.834 relate the $\mathrm{V}$ band extinction to the $g$ and $r$ bands assuming a Milky Way dust model. The corrected equivalent width is obtained as follows

$$
w=w_{0}\left[(1+z) \times 10^{-0.4\left(0.775 \mathrm{~A}_{\mathrm{V}, l}\right)(1-1 / f)}\right]^{-1}
$$


where $w_{0}$ is the measured, uncorrected equivalent width. The $1+z$ arises from the fact that the total flux in the Ha line is not affected by cosmological expansion of the universe but the flux per unit wavelength of the continuum is depressed by a factor of $1+z$. The following term is the extinction correction. The 0.775 relates the $\mathrm{V}$ band extinction to the $\mathrm{H} \alpha$ line assuming a Milky Way dust model and the $1-1 / f$ is due to the fact that the emission line and continuum experience different amounts of extinction as previously explained.

Figure 2.1 shows the distribution of the galaxies in the color vs. $\mathrm{H} \alpha \mathrm{EW}$ plane.

\subsubsection{Errors}

In order to conduct a likelihood analysis we need error estimates which take into account both the errors induced by the photometry and spectroscopy and those by the aforementioned corrections. The error in the corrected color, $\sigma_{c}$, is given by

$$
\sigma_{c}=0.03+\sqrt{\sigma_{g}^{2}+\sigma_{r}^{2}+\left(\frac{0.319 \mathrm{~A}_{\mathrm{V}, l}}{f}\right)^{2}\left(\left(\frac{\sigma_{\mathrm{A}_{\mathrm{V}, l}}}{\mathrm{~A}_{\mathrm{V}, l}}\right)^{2}+\left(\frac{\sigma_{f}}{f}\right)^{2}\right)+\left(0.0440 \mathrm{~A}_{g}\right)^{2}+\sigma_{k}^{2}}
$$

where $\sigma_{g}$ and $\sigma_{r}$ are the Poisson errors in the observed $g$ and $r$ band photometry, $f$ is the ratio of the emission line to continum extinction, and $\sigma_{k}$ is the error introduced by the $K$-corrections. The terms inside the square root in equation 2.4 are obtained through a straight error propagation of equation 2.2. The 0.03 outside the square root is due to the systematic zero point errors of the SDSS filter system. Following Calzetti, Kinney, \& Storchi-Bergmann (1994) $f$ is fixed at 2.0 and $\sigma_{f}$ is set to 0.4 . The error in emission line $A_{V}$ is given by

$$
\sigma_{\mathrm{A}_{\mathrm{V}, l}}^{2}=9.440\left(\left(\frac{\sigma_{\mathrm{H} \alpha}}{\mathrm{H} \alpha}\right)^{2}+\left(\frac{\sigma_{\mathrm{H} \beta}}{\mathrm{H} \beta}\right)^{2}\right)
$$


which is dependent on the fractional uncertainty of the $\mathrm{H} \alpha$ and $\mathrm{H} \beta$ emission line fluxes. Schlegel, Finkbeiner, \& Davis (1998) reports a $16 \%$ error in their Milky Way reddening values. Because a dust model is assumed reddening in the $g$ and $r$ bands are linearly related, so the error in $\mathrm{A}_{g}-\mathrm{A}_{r}$ is a function of $\mathrm{A}_{g}$. This relationship combined with the $16 \%$ error yields the 0.0440 in equation 2.4. The median value of $g$ band reddening is 0.10 so the errors introduced by the MW reddening correction are insignificant for the majority of objects. Errors in the redshift determination are negligible, typically $0.01 \%$. The value of $\sigma_{k}$ is estimated to be 0.02 by visual inspection of a plot of $k_{g}-k_{r}$ as a function of redshift.

For a typical galaxy the term involving $\sigma_{f}$ is the largest contributor to the extinction corrected color error. The median Poisson error from the photometry is 0.01 in both bands. The median value of $\sigma_{c}$ is 0.085 .

The error in the corrected $\mathrm{H} \alpha$ equivalent width, $\sigma_{w}$, is given by

$$
\sigma_{w}^{2}=w^{2}\left[\left(\frac{\sigma_{w_{0}}}{w_{0}}\right)^{2}+0.5095\left(\left(\sigma_{\mathrm{A}_{\mathrm{V}, l}}(1-1 / f)\right)^{2}+\left(\frac{\sigma_{f} \mathrm{~A}_{\mathrm{V}, l}}{f^{2}}\right)^{2}\right)\right]
$$

Equation 2.6 is the result of propagating the errors in equation 2.3, neglecting the insignificant redshift errors. Again, the term involving $\sigma_{f}$ is the largest contributor to the error for typical galaxies. The median error in the equivalent width is $17 \%$. The median error bars for the sample are shown in Figure 2.1. For comparison, K83 reports equivalent width errors of around $10 \%$, but the extinction is uncertain at the $20-30 \%$ level.

\section{$2.3 \quad$ Spectral Synthesis Models}

In order to interpret the data it is necessary to employ the use of models. Spectral synthesis models predict the spectra of galaxies based on a host of input parameters. In this 
project PEGASE (Projet d'Etude des GAlaxies par Synthèse Evolutive) version 2.0 models are used (Fioc \& Rocca-Volmerange 1997). The idea behind spectral synthesis models is very simple, although the computational implication is not as straightforward. The greatest limitation is that these models are at best only as good as the spectral templates and dust models that go into them.

The flux of a model galaxy at time $t$ and wavelength $\lambda$ is given by

$$
F_{\lambda}(t)=\int_{0}^{t} \int_{m_{l}}^{m_{u t}} \tau(t-\theta) \phi(m) f_{\lambda}(m, \theta) \mathrm{d} m \mathrm{~d} \theta
$$

where $\tau(t-\theta)$ is the SFR at time $t-\theta, \phi(m)$ in the IMF and $f_{\lambda}(m, \theta)$ is the flux of a star of mass $m$ and age $\theta$ at wavelength $\lambda$. The mass is integrated over $m_{l}$ to $m_{u}$, the lower and upper mass cutoffs of the IMF.

Both integrals cannot be discretized as this leads to oscillations in the resulting spectra due to short lived evolutionary phases like the asymptotic giant branch. One method to get around this is the isomass method where the mass integral is discretized. The other, the isochrone method, discretizes the time integral. Fioc \& Rocca-Volmerange (1997) found that both methods give the same results but they prefer the isochrone methods in part because isochrones can be compared directly to color magnitude diagrams of star clusters. PEGASE works by first calculating the spectrum of an instantaneous burst of star formation for a given IMF at varying times. The galaxy spectrum returned by PEGASE is a. convolution of instantaneous starbursts of different ages and strengths detcrmined by the star formation history.

Fioc \& Rocca-Volmerange (1997) note that the spectral synthesis models of most authors are in good agreement in the visual. In the NIR the discrepancies become significant. 
The main uncertainties in the PEGASE are due to the uncertainties in stellar evolutionary tracks, especially as a function of metallicity. Effects of metallicity in the stellar spectra. themselves is also a concern. They also point out that metallicity can influence extinction curves. This problem affects the extinction correction of the sample in our case as the PEGASE models computed here are all extinction free.

The two principal inputs into the models are the IMF and the SFH. The IMF indicators of present interest are insensitive to the IMF at low masses so the IMF was parameterized as follows

$$
n_{\log m} \propto\left\{\begin{array}{ccc}
0.5 & \text { for } & 0.1<m / \mathrm{M}_{\odot}<0.5 \\
\Gamma & \text { for } & 0.5<m / \mathrm{M}_{\odot}<120
\end{array}\right.
$$

following Baldry \& Glazebrook (2003). Models were calculated for $1.00 \leq \Gamma \leq 2.00$ in increments of 0.05 in $\Gamma$.

In the initial analysis models were calculated only for SFHs which are continuous and quiescent. Figure 2.2 shows the SFHs considered. These include an instantaneous burst at $t=0$, SFRs $\propto e^{-t / \tau}$ where $\tau=1.10,1.40,1.55,1.70,1.85,2.00,2.15,2.35,2.50,2.75,3.0$, $3.5,4.0,5.0,6.0,7.5,10,15$ and $35 \mathrm{Gyr}$, a constant SFH, and increasing SFHs $\propto 1-e^{-t / \tau}$ where $\tau=2.0,4.0,7.5$ and $15 \mathrm{Gyr}$. In $\S 3.1 .3$ models with star formation discontinuities, bursts and gasps, are calculated and discussed.

There are several other inputs to the PEGASE models. In our models stars are formed at a constant solar metallicity. PEGASE allows for cvolving stcllar metallicitics. To calculate this it relies in part on the user specified fraction of close binary systems to predict the number of type Ia supernovae, but in our case this is irrelevant. PEGASE also allows for galaxies to form by infall of gas although ours are based on a galaxy that 
stars out fully constituted. Galactic winds can expel the ISM from the galaxy ending star formation. This feature is turned off. The mass fraction of substellar objects locks up mass in objects which do not contribute to the integrated light. We set this to 0 as well as our IMF is truncated at $0.1 \mathrm{M}_{\odot}$. Lastly PEGASE has the option, which is used, of calculating nebular emission which is important because we need to predict the $\mathrm{H} \alpha$ equivalent width. PEGASE determines the nebular emission from the strength of the Lyman continuum due to the photospheres of massive stars. The UV flux is converted to $\mathrm{H} \alpha$ flux assuming Case $\mathrm{B}$ combination. The strengths of other emission lines are calculated relative to the $\mathrm{H} \alpha$ flux using user defined line ratios. It does not take into account the metallicity of the ISM in this process and only gives the line strengths explicitly specified by the user.

In total this forms a grid of $21 \mathrm{IMFs}$ by $25 \mathrm{SFHs}$ where all other inputs are the same. Figures 2.3, 2.4, 2.5, and 2.6 demonstrate how changes in parameters affect model galaxy spectra. In all three figures the blue spectrum is that of a galaxy with a constant SFR and $\Gamma=1.35$ at an age of $12 \mathrm{Gyr}$.

Figure 2.3 shows a galaxy with a constant SFR and $\Gamma=1.35$ as it would appear at ages from $100 \mathrm{Myr}$ to $20 \mathrm{Gyr}$. Perhaps the most important feature of this plot is that it shows that for a given SFR the UV flux is the nearly the same regardless of the age. It is also obvious that the galaxy becomes much redder as it ages. This is because lower mass stars have much longer lifetimes than young UV luminous stars. The total mass in stars continually increases, but the number of high mass star's stays the same. Because the continuum is much redder the $\mathrm{H} \alpha \mathrm{EW}$ is reduced although the SFR stays the same. This is why it is important, that galaxies with recent bursts be rejected from the sample. 
Figure 2.4 shows spectra for galaxies with the same IMF and age of $12 \mathrm{Gyr}$, but different SFHs. The SFHs have been normalized to form an equal mass of stars at 12 Gyr. Although not as tight of a correlation as the relationship between UV flux and SFR, it can be seen that galaxies with equal stellar mass have similar NIR flux. Figure 2.4 also shows the relationship between the SFR and the strength of emission lines. By comparing figure 2.4 to figure 2.3 it can be seen that changes in the $\mathrm{SFH}$ of a galaxy can have very similar effects as changes in the age.

Figure 2.5 shows galaxies with equal constant SFRs at ages of 12 Gyr where the IMF slope is allowed to vary. The IMF is that of equation 2.8 where $\Gamma$ ranges from 1.0 to 2.0. From comparison with figure 2.4 it is obvious that changing the IMF has a very similar effect to changing the SFH. Because steeper IMFs form a larger fraction of mass into massive stars it can appear that the SFR is higher. This is the most difficult challenge of this project. This figure also shows galaxies with equal stellar mass have similar NIR fluxes.

The model parameter of interest is the IMF. Implicit in equation 2.8 is the assumption that the IMF does not vary as a function of time.

The continua of galaxies are weakly influenced by low mass stars in the optical. This method is sensitive to the IMF for masses above around $1 \mathrm{M}_{\odot}$ so the slope is fixed below $0.5 \mathrm{M}_{\odot}$. Above $0.5 \mathrm{M}_{\odot}$ models are calculated for $1.00 \leq \Gamma \leq 2.00$, where $\Gamma$ is incremented by 0.05 between models.

We treat our IMF model as though it has only one degree of freedom-- $\Gamma$ above 0.5 $\mathrm{M}_{\odot}$. In truth it has three more as written: the lower and upper mass cutoffs and the point 
at which the slope changes. So how well are our assumptions justified?

We have parameterized the IMF as a piecewise power law with two components. Piecewise power laws are motivated by empirical fits to data starting with Salpeter (1955), which had only one component. By contrast the power law formulation of the Scalo (1986) IMF has 24 components. The log-normal distribution is a more physical choice as it can arise from stochastic processes. Miller \& Scalo (1979) were the first to fit an observational measurement of the IMF with a log-normal distribution. The log-normal distribution is normalizable as it goes to zero smoothly at both extremes without any awkward truncation. Its main drawback is that it cannot fit any structure in the IMF over small mass ranges.

Log-normal distributions have three degrees of freedom. This is less than the four our model has. However, we are not sensitive to IMF over the full range of masses which makes it much more difficult to fit the parameters of the log-normal distribution. Instead we use this piecewise model and lower the degrees of freedom through physical arguments. Many investigators find a change in slope in the IMF around $0.5 \mathrm{M}_{\odot}$. Our fixed lower end of the IMF is designed to be consistent to this. As our technique is not sensitive to this regime this assumption does not impact the results.

The IMF must be normalizable because the total mass of a stellar population is finite. In our parameterization this is achieved by truncation at 0.1 and $120 \mathrm{M}_{\odot}$. This seems unphysical as the existence of brown dwarfs suggests that the IMF should continue below the hydrogen burning limit. However, stars at $0.1 \mathrm{M}_{\odot}$ do not contribute much to the integrated light of galaxies. As we are not sensitive to stars in this mass range this choice is not unreasonable. In fact, truncating the IMF at $0.5 \mathrm{M}_{\odot}$ yields models which are at worse 
differ by 0.002 in $(g-r)_{0.1}$ and $3 \%$ in $\mathrm{H} \alpha$ EW from those with low mass stars. The slope below $0.5 \mathrm{M}_{\odot}$ has essentially no effect on our results. Only when the IMF is truncated at $0.9 \mathrm{M}_{\odot}$ do the models differ at the level of the errors in the data.

On the high mass end the choice of limit does matter. There is a physical upper limit to the size of stars associated with the Eddington limit. The value of this theoretical limit is not widely agreed upon. The largest stellar mass measured reliably, via analysis of a binary system, is $83 \pm 5 \mathrm{M}_{\odot}$ (Bonanos, et al. 2004). Weidner \& Kroupa (2004) argue that given the large mass and youth of the star forming cluster R136 in the Large Magellanic Cloud stars in excess of $750 \mathrm{M}_{\odot}$ should be present given a Salpeter IMF with no upper mass limit to stars, whereas no stars above $150 \mathrm{M}_{\odot}$ are observed. An analysis of the Arches Cluster, the youngest observable cluster, gives an upper limit of $150 \mathrm{M}_{\odot}$ based on Monte Carlo simulations although stars above $130 \mathrm{M}_{\odot}$ are not detected (Figer 2005). The PEGASE model tracks only extend up to $120 \mathrm{M}_{\odot}$ so this is the cutoff used. Another issue is that the physics and evolution of such high mass stars is not well known so the models themselves may be a significant source of error in this regime.

The left half of Figure 2.7 shows the effects of varying the high mass cutoff in the IMF. The effect in the $(g-r)_{0.1}-\mathrm{H} \alpha \mathrm{EW}$ plane is seen to be very similar to increasing the value of $\Gamma$. Lowering the upper mass cutoff from 120 to $90 \mathrm{M}_{\odot}$ has roughly the same effect as increasing $\Gamma$ from 1.35 to 1.45 . The relationship between the change in the upper mass culoff (from $120 \mathrm{M}_{\odot}$ ) and the apparent change in $\Gamma$ is $\Delta \Gamma \sim 0.005 \Delta \mathrm{M}_{\mathrm{up}}$ and is roughly linear for upper mass cutoffs down to $50 \mathrm{M}_{\odot}$. The coefficient in the relationship is a week function of the age of the population ranging from 0.004 for $13 \mathrm{Gyr}$ old populations to 0.006 
for $100 \mathrm{Myr}$ old populations.

The right half of Figure 2.7 shows the affect of adding a second break in the IMF at $10 \mathrm{M}_{\odot}$. Reducing the value of $\Gamma$ over the $0.5-10 \mathrm{M}_{\odot}$ range while keeping it fixed at $\Gamma=1.35$ above $10 \mathrm{M}_{\odot}$ has a similar effect to decreasing $\Gamma$ in a two component model. This illustrates one of the limitations of this model. At this point it is not possible to detect fine structure in the IMF slope or to state precise values for the IMF slope. In this limited space of observables the IMF models themselves are degenerate. What it does provide is a framework with which to detect variations in the IMF. Although we can construct similar tracks from different IMF models, we can still detect the differences between two groups of galaxies.

While we will report our results as a function of $\Gamma$ it must always be kept in mind that it is degenerate with the upper mass cutoff and other fine structure in the IMF at high stellar masses.

The assumption of smoothly varying SFHs is of great consequence. In the event that a galaxy is experiencing or has recently experienced a burst our SFH assumption can lead to measured $\Gamma$ values that are off by as much as 0.5 . The effects of bursts are more closely examined in a later section.

Within the assumption of smoothly varying SFHs much can be said about the effects of the IMF, metallicity, age, and SFH in the color-H $\alpha$ EW plane. Figure 2.8 demonstrates these relationships. In both panels the ages of the models decrease from the upper left to lower right. The effects of the age of a stellar population are largely orthogonal to those of IMF variations. In Figure 2.8a the effects of changing the functional form of the 
smoothly varying SFH with fixed metallicity and $\Gamma$ are shown. SFH variation is degenerate with the IMF. However the effect is relatively small over a wide range of SFHs. The solid lines are exponentially decreasing SFHs with $\tau=1.1 \mathrm{Gyr}$ where the bulk of the star formation occurs early in the galaxy's life. The dashed lines have SFHs that are increasing with time where most star formation occurs at late ages. The effect of variations in the form of smooth SFHs is larger at later ages and higher values of $\Gamma$ but does not dominate the effects of IMF variations. With all other parameters fixed the range of smooth SFHs cause systematic uncertainties at the level of \pm 0.1 in $\Gamma$.

In Figure 2.8b the effects of metallicity variations with fixed SFH and $\Gamma$ are shown. The metallicity variations are also degenerate with IMF variations. With all other parameters fixed between colors of $0.1<(g-r)_{0.1}<0.4$ the systematic uncertainty due to metallicity is less than 0.05 in $\Gamma$. This uncertainty increases to 0.35 at $(g-r)_{0.1}=-0.2$ and 0.7 .

As aforementioned, the extinction correction is another potential problem. The arrows in Figure 2.8 show the length and direction of the extinction correction for typical galaxies in our sample. It is assumed that $f=2$, but $f=1$ and $f=4$ are also plotted to show the potential effect of variations in $f$. The reddening vectors for $f=2$ and 4 are fortuitously orthogonal to the IMF variations. Only when the continuum and emission extinctions are equal, when $f=1$, do the extinction correction and variations in $f$ become a largor concern than metallicity and SFH. However such low f ratios are not observed in galaxies (Calzetti, Kinney, \& Storchi-Bergmann (1994) and section 3.1.2).

Figure 2.9 shows all 18,480 model points with $\Gamma=1.35$. Models are interpolated 
in SFH history for fuller coverage of the color- $\mathrm{H} \alpha \mathrm{EW}$ plane. For each value of $\Gamma$ the models cover a stripe rather than a single line. It can be seen in the lower right of figure 2.9 that the model become degenerate in $\Gamma$ for old, red galaxies with weak current star formation.

\subsection{Statistical Techniques}

The data and models are compared using a "pseudo- $\chi$ " minimization. For various reasons (detailed below) the classical $\chi^{2}$ estimator assumptions are violated so we can not use traditional tables for error estimates but we can still use the $\chi^{2}$ as a statistical estimator as long as the confidence regions are calibrated by Monte Carlo (MC) techniques as we will do. We proceed as follows: for each galaxy $i$ we have a measured $(g-r)_{0.1}$ color, $c_{i}$, and an $\mathrm{H} \alpha \mathrm{EW}, w_{i}$, and measurement errors $\sigma_{c_{i}}$ and $\sigma_{w_{i}}$, given by equations 2.4 and 2.6. We also have model values $c(\Gamma, Z, \tau, \psi)$ and $w(\Gamma, Z, \tau, \psi)$ for a range of IMF slopes $\Gamma$, metallicities $Z$, ages $t$ and SFHs $\psi$. We can then construct a $\chi^{2}$ value as

$$
\chi_{i}^{2}(\Gamma, Z, t, \psi)=\left(\frac{c_{i}-c(\Gamma, Z, t, \psi)}{\sigma_{c_{i}}}\right)^{2}+\left(\frac{w_{i}-w(\Gamma, Z, t, \psi)}{\sigma_{w_{i}}}\right)^{2}
$$

which is calculated by brute force. The goal of this paper however is to investigate the IMF with relatively simple measurements of the $\mathrm{H} \alpha \mathrm{EW}$ and a broadband color. While making crude measurements of the mean stellar metallicities of individual galaxies is possible, disentangling age and SFH effects on an individual basis is a daunting task. Assuming that it is possiblo to do, it does not scale up well to the high redshift universe where obscrvations will be of lower quality.

It does not make sense to minimize $\chi^{2}$ over all galaxies for a particular set of $(\Gamma, Z, t, \psi)$ because we have no a priori reason to think that all of the galaxies should have 
the same metallicity or SFH. In fact we expect that they would not. The solution is to marginalize $\chi^{2}$ over metallicity, age and SFH for each galaxy such that

$$
\chi_{i}^{2}(\Gamma)=\min \left[\chi_{i}^{2}(\Gamma, Z, t, \psi)\right]_{Z, t, \psi}
$$

This is somewhat unorthodox, because for some galaxies the data points are overfitted, i.e. there will be a stripe in $(c, w)$ space corresponding to a given $\Gamma$ and we can get $\chi_{i}^{2}(\Gamma)$ values very close to zero (but not exactly because of the discrete nature of the model grid) for galaxies within the stripe. We note we also have partial degeneracy between parameters such as age and metallicity - they both shift the tracks in similar directions largely orthogonal to $\Gamma$ (though not completely which is why we have a stripe in parameter space not a line). This makes it difficult to calculate the traditional "number of degrees of freedom." Despite these limitations it is clear that galaxies inconsistent with a particular $\Gamma$ will still have large values of $\chi_{i}^{2}(\Gamma)$ - for example a very blue galaxy with a low equivalent width in Figure 2.9. The complication is that the stripes for similar $\Gamma$ values overlap, and for red galaxies with low equivalent widths the stripes for vastly different $\Gamma$ values overlap. As such, the IMF for an individual galaxy is only broadly constrained. Measuring a precise best IMF for an individual galaxy boils down to random chance and the discrete nature of the models. However, by summing $\chi_{i}^{2}$ over many galaxies the IMF is narrowly constrained for the sample being summed over as long as we are careful in our confidence region analysis.

Becanse of this over-fitting and partial degeneracy we can not apply the texthook notions of the $\chi^{2}$ distribution, calculate degrees of freedom and choose $\Delta \chi^{2}$ contours for different confidence regions. Further to this $c_{i}$ and $w_{i}$ are not truly independent variables. Both the colors and EWs are subject to the same extinction and reddening corrections 
which tie the errors together. For galaxies with $z \lesssim 0.04$ the $\mathrm{H} \alpha$ line is in the observed $r$ band, although this only affects a relatively small number of galaxies in the sample almost all of which have $M_{r, 0.1}>-20$. Also the direct statistical interpretation of $\chi^{2}$ is predicated on the assumption of normal errors. Equations 2.4, 2.5 and 2.6 reveal that our errors are complicated mixtures of individual measurements which are most likely Poisson distributed. Thus $\sigma_{c}$ and $\sigma_{u}$ are unlikely to be normally distributed. Bursty SFHs can potentially create outliers which are statistically significant due to the fact that neither $\sigma_{c}$ or $\sigma_{w}$ include a term for this difficult to quantify effect. The problem is even worse if the errors are nonsymmetric which could potentially arise from the aforementioned bursty SFHs. In the case of non-symmetric errors the best value of $\Gamma$ could erroneously be pulled away from the true value.

Given all this we abandon the direct statistical interpretation of $\chi^{2}$ and regard it as an estimator of the goodness of fit whose confidence regions have to be calibrated empirically. We do this via MC simulations (as recommended by Press, et al. (1992)) where we simulate data points for a given $\Gamma$ with the correct error distributions and propagate everything through the analysis in the same way as for the actual data. For each of our MC simulations we add Poisson errors to the ugriz fiber magnitudes, $\mathrm{H} \alpha$ and $\mathrm{H} \beta$ fluxes and the observed Ho EW. We assume that these observed quantities have Poisson dominated errors- as members of the MGS they are high $\mathrm{S} / \mathrm{N}$ measurements. The entire analysis described above is repeated, including a new extinction measurement and a recalculation of the $K$-corrections. For each value of $\Gamma$ we run $100 \mathrm{MC}$ simulations to estimate the $95 \%$ confidence interval. Setting up the $\mathrm{MC}$ architecture in this way has the further advantage 
that we can use the same machinery to test the effect of systematic errors such as the violation of our smooth SFH assumptions, as we will do later. The main downside of course is that this approach is computationally intense. Run times for the $100 \mathrm{MC}$ simulations are typically 18 days on a $2 \mathrm{Ghz}$ desktop PC for the samples considered here.

In practice it turns out that $\chi^{2}(\Gamma)$ is still a smooth well-behaved function with, not surprisingly, a quadratic minimum which has the advantage that we can then interpolate it to increase the resolution in the best-fitting $\Gamma$ without incurring the additional computational expense. This arises of course from the fact that our estimator is similar to a traditional $\chi^{2}$ and is a good reason to stick with this similarity over some more exotic, goodness of fit measure. An estimate of the systematic errors is discussed later.

Regardless of how poorly a sample is modeled by a universal IMF the above method will still find a best fitting $\Gamma$ and corresponding confidence region. We still expect $\chi^{2}$ to be small for a model that is a good fit and large for one that is not. One nuance in comparing $\chi^{2}$ between different sub-samples, as we will do, is that the samples are often of considerably different sizes. Because of this we choose instead to use the mean $\chi^{2}, \overline{\chi^{2}}$ instead, as a sample metric. This has the advantage that absolute $\overline{\chi^{2}}$ values and confidence regions are more similar between the sub-samples, though we note that the confidence regions on $\overline{\chi^{2}}$ are still determined directly from our MC simulations. 


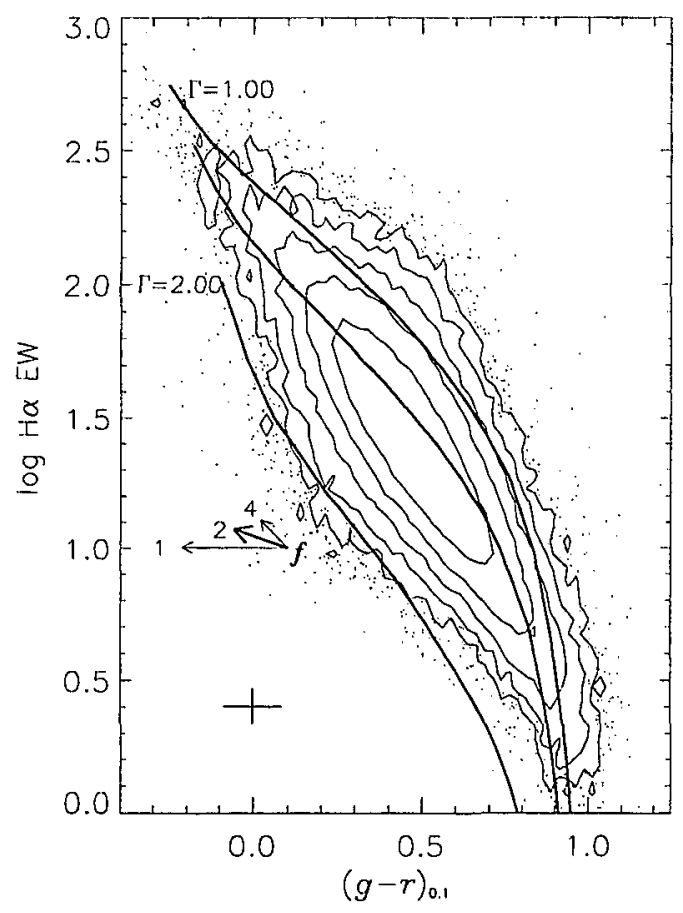

Figure 2.1 Distribution of all 130,602 program galaxies in the $(g-r)_{0.1}$ vs. $\log (\mathrm{H} \alpha \mathrm{EW})$ plane. The contours are logarithmic. Outside the last contour individual points are plotted. The thick lines are model tracks with exponentially decreasing SFHs with $\tau=1.1 \mathrm{Gyr}$ and solar metallicity. The age increases along the tracks from $100 \mathrm{Myr}$ in the upper left to 13 Gyr in the lower right. The upper line has $\Gamma=1.00$, the middle line is similar to Salpeter's IMF with $\Gamma=1.35$ and the lower line has $\Gamma=2.00$. They are identical to the solid lines in Figure 2.8. The cross in the lower left indicates the median error bars of the sample. The arrows are dust vectors are for typical observed Balmer decrements $(\mathrm{H} \alpha / \mathrm{H} \beta=4)$ for different values of $f$. The values of $f$ are 1,2 and 4 clockwise from the one pointing left. 


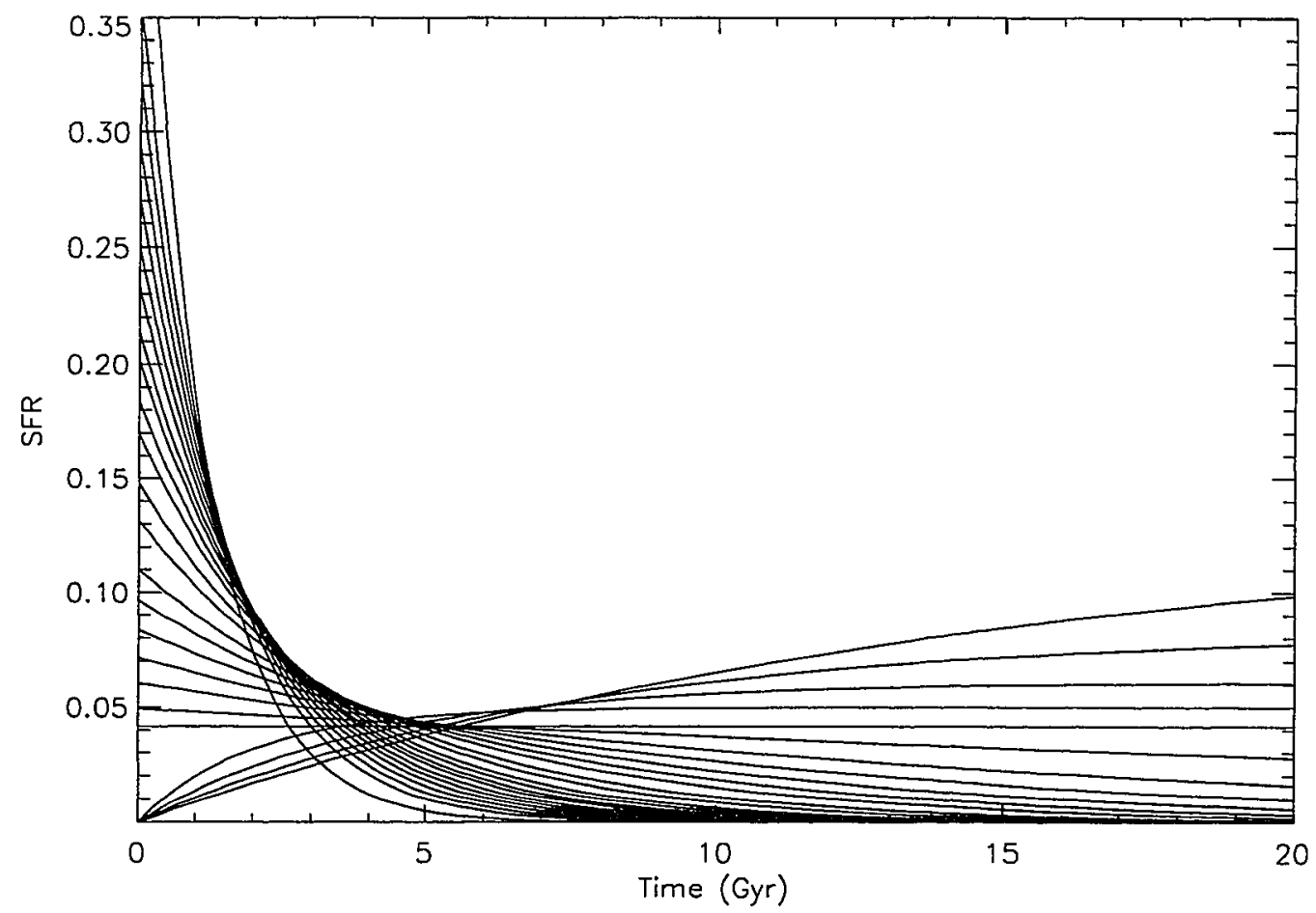

Figure 2.2 Star formation histories for which models were calculated. The SFH have been normalized such that an equal mass of stars is formed by an age of $12 \mathrm{Gyr}$. Not pictured is an instantaneous burst at $t=0$. Plotted are SFHs $\propto e^{-t / \tau}$ where $\tau=1.10,1.40,1.55,1.70$, $1.85,2.00,2.15,2.35,2.50,2.75,3.0,3.5,4.0,5.0,6.0,7.5,10,15$ and 35 Gyr, a constant $\mathrm{SFH}$, and increasing SFHs $\propto 1-e^{-t / \tau}$ where $\tau=2.0,4.0,7.5$ and 15 Gyr. 


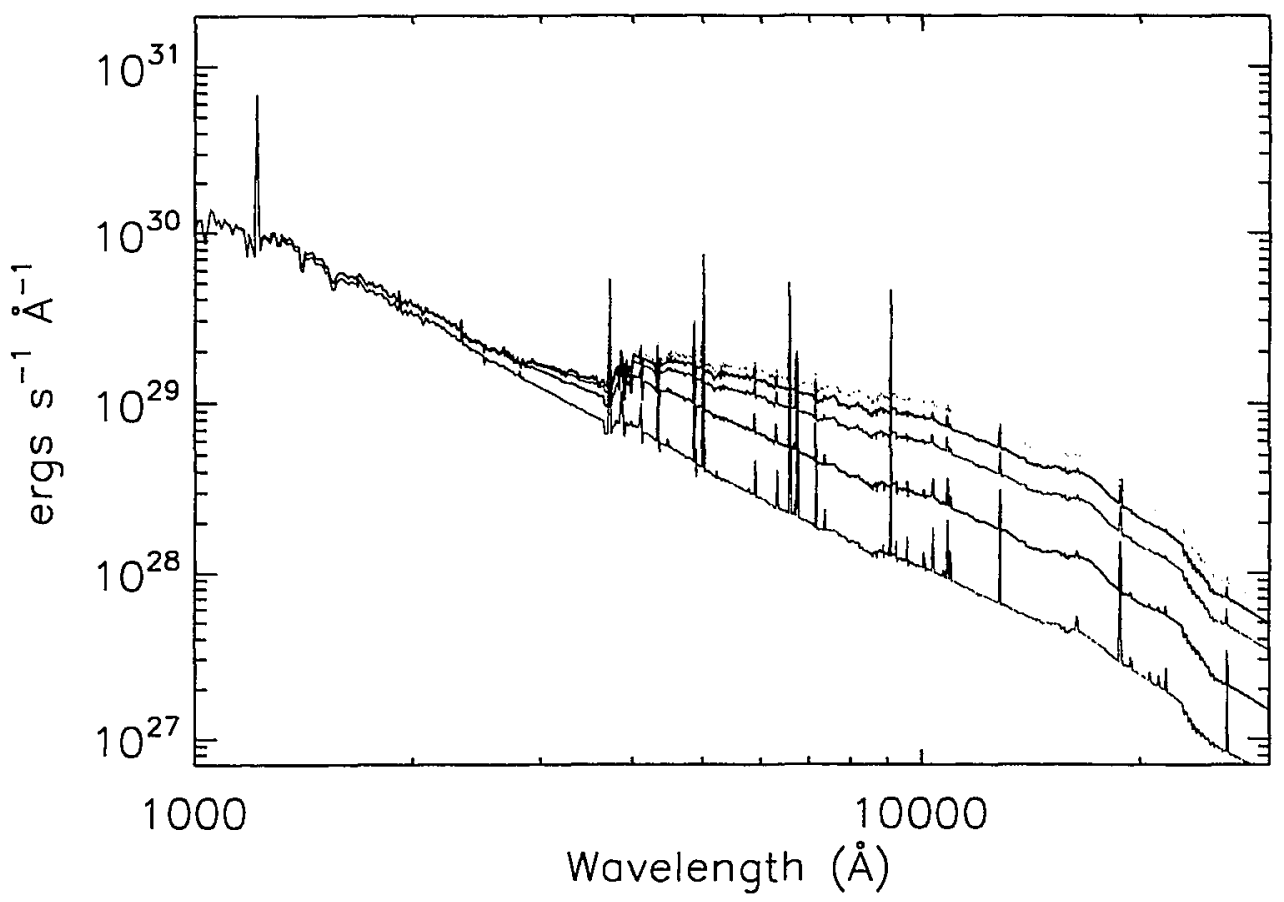

Figure 2.3 PEGASE model spectra of a galaxy at $z=0.2$ with a constant SFH and $\Gamma=1.35$ as it would appear with increasing age. The galaxy is shown at ages $100 \mathrm{Myr}$ (red), 1 Gyr (green), $5 \mathrm{Gyr}$ (cyan), $12 \mathrm{Gyr}$ (blue) and $20 \mathrm{Gyr}$ (violet). 


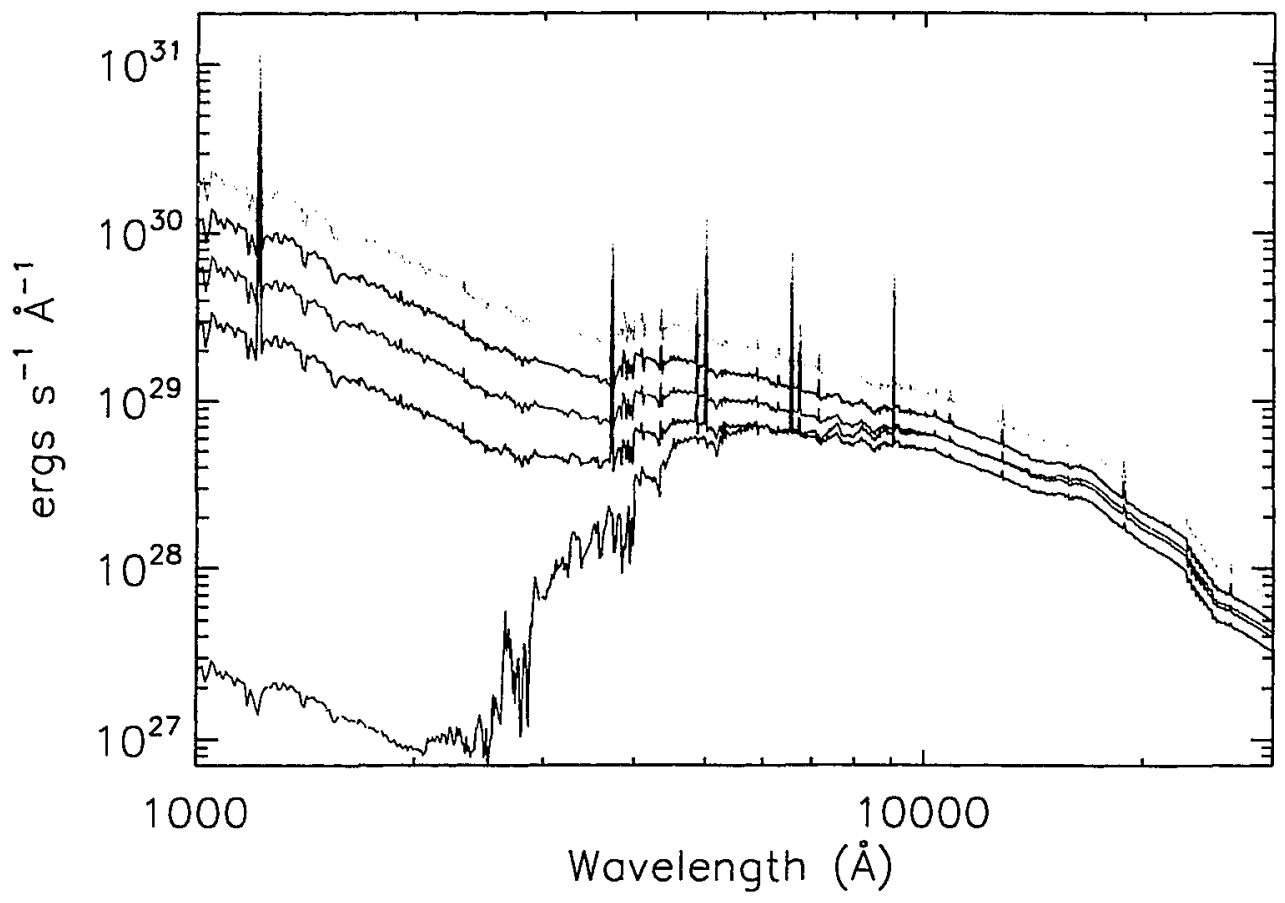

Figure 2.4 PEGASE model spectra of galaxies at $z=0.2$ with $\Gamma=1.35$ at an age of 12 Gyr. The galaxies differ in their SFHs. Spectra are plotted for an instantaneous burst at $t=0$ (red), SFH $\propto e^{-t / \tau}$ where $\tau$ equals $2 \mathrm{Gyr}$ (orange), $5 \mathrm{Gyr}$ (green) and $10 \mathrm{Gyr}$ (cyan), a constant SFR (blue) and an increasing SFR $\propto 1-e^{-t / \tau}$ where $\tau$ is 15 Gyr (violet) 


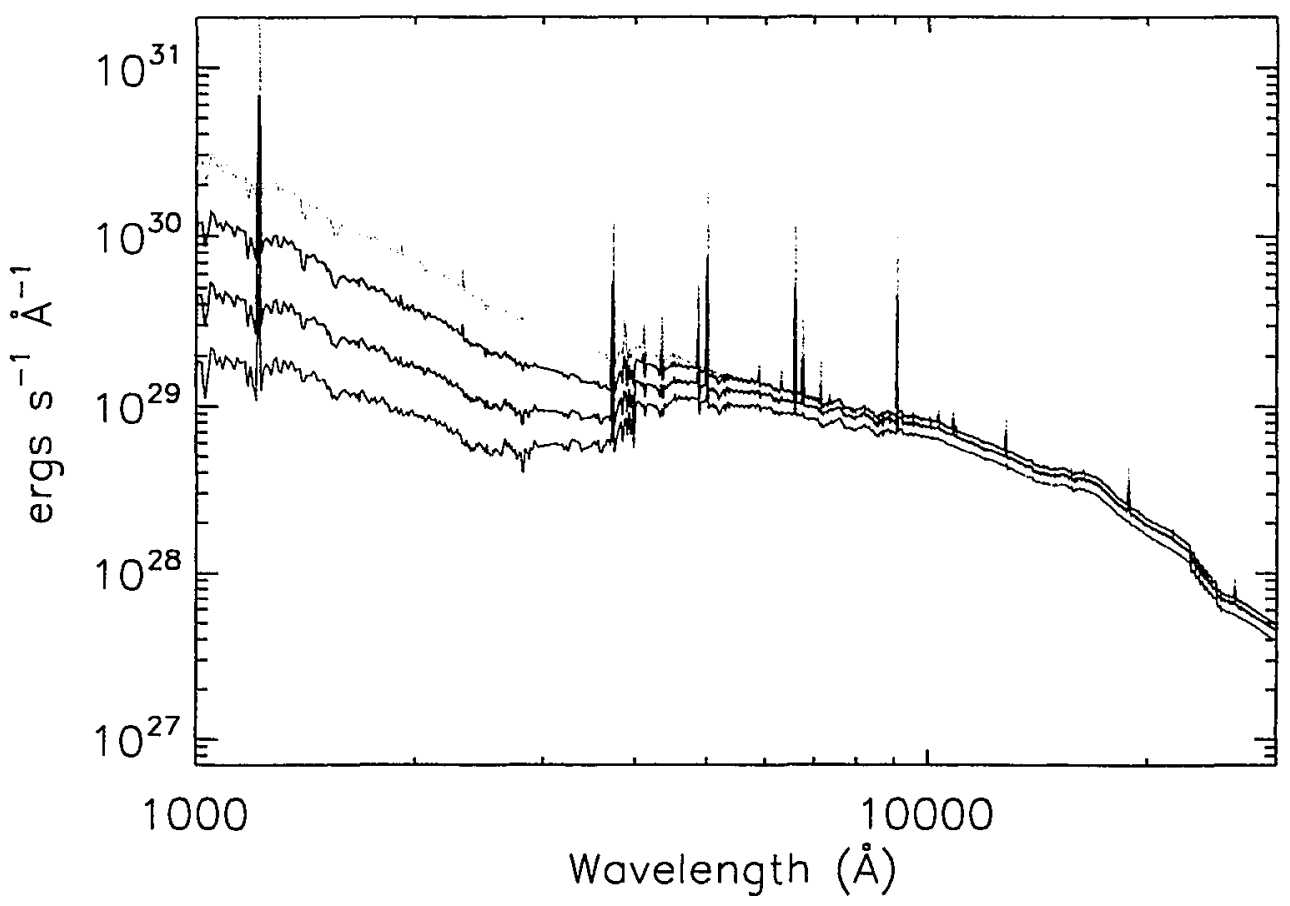

Figure 2.5 PEGASE model spectra of galaxies at $z=0.2$ with ages of $12 \mathrm{Gyr}$ and constant SFRs, but different IMF slopes. The IMF is of the form of equation 2.8 where $\Gamma$ takes the values 1.00 (violet), 1.35 (blue), 1.70 (green) and 2.00 (red). 


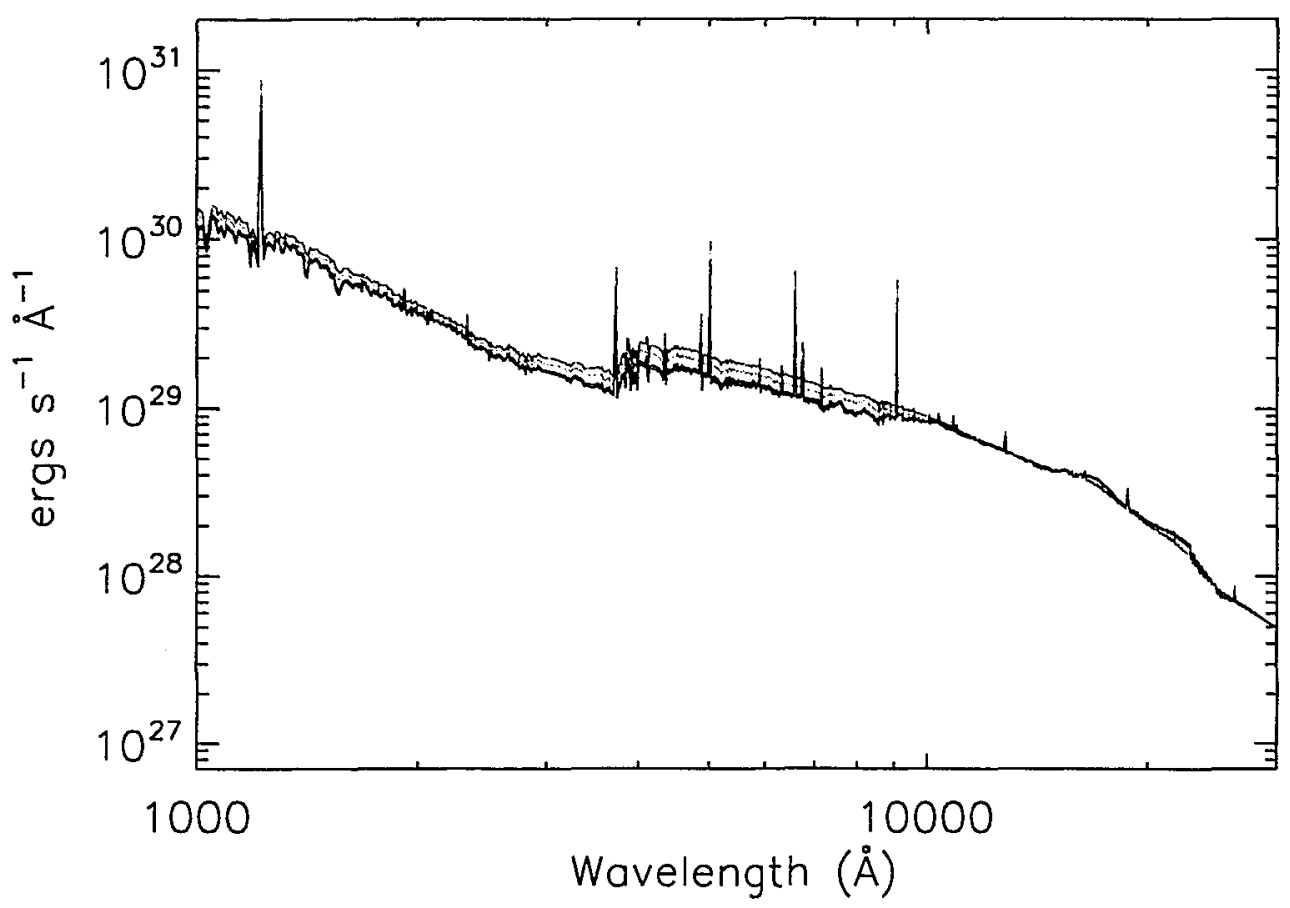

Figure 2.6 PEGASE model spectra of galaxies with constant SFRs, $\Gamma=1.35$, and an age of 12 Gyr where the metallicity varies between models. The metallicities are $Z=0.005$ (red), 0.010 (violet), 0.020 (blue), and 0.025 (green). 


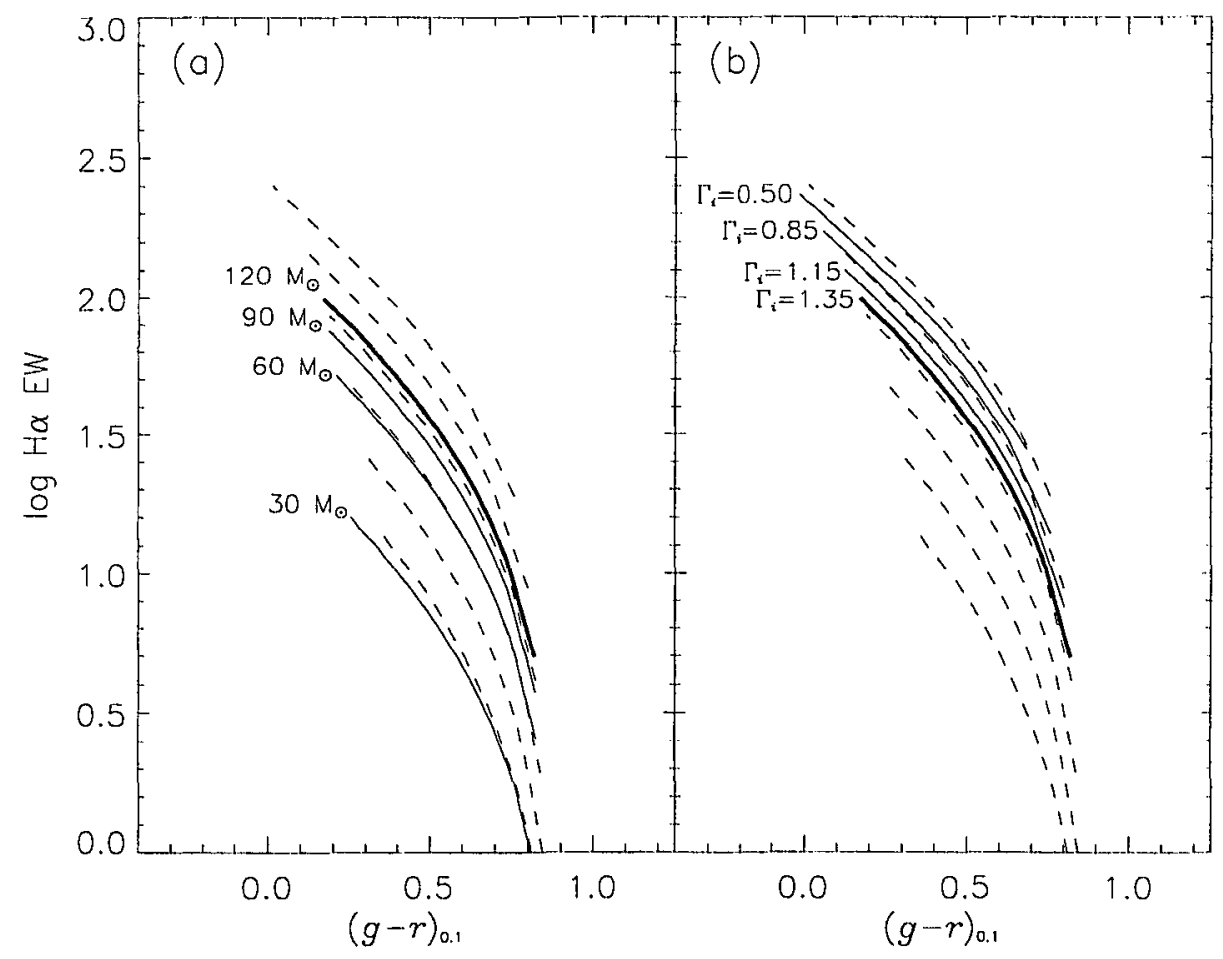

Figure 2.7 Examples of the degeneracy of IMF parameters. All models shown have solar metallicity, age 6 Gyr and the tracks are lines of varying SFH from a rapidly decreasing SFR on the lower right to slowly increasing on the upper left. In both panels the bold track has $\Gamma=1.35$ and the dashed lines have IMFs $\Gamma=1.00,1.20,1.40,1.60,1.80$ and 2.00 from top to bottom, all with an upper mass cutoff of $120 \mathrm{M}_{\odot}$. (a) The solid lines are models where the upper mass cutoff of the IMF is reduced from $120 \mathrm{M}_{\odot}$ to 90,60 and $30 \mathrm{M}_{\odot}$ as labeled. (b) The nominal two-piece IMF is replaced by a three piece IMF in the solid lines. In all solid tracks $\Gamma=1.35$ above $10 \mathrm{M}_{\odot}$ and $\Gamma=0.50$ below $0.5 \mathrm{M}_{\odot}$, but is altered in the intermediate mass region from 0.5 to $10 \mathrm{M}_{\odot}$. The solid lines have $\Gamma_{i}=0.50,0.85$ and 1.15 over 0.5 to $10 \mathrm{M}_{\odot}$ as labeled. 


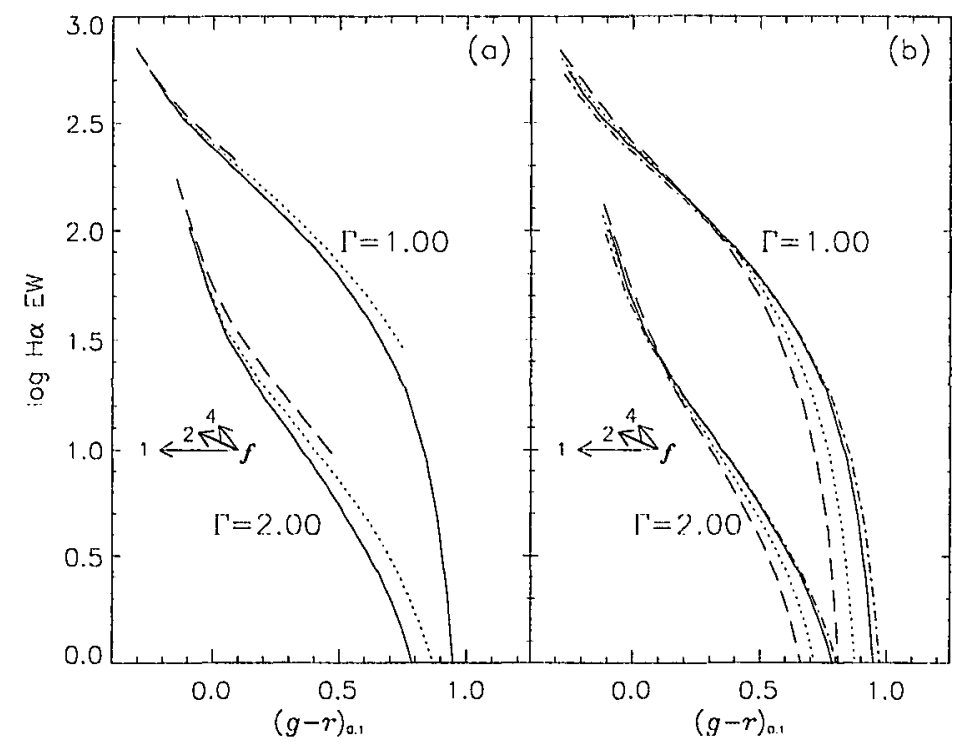

Figure 2.8 Effects of model parameters in the $(g-r)_{0.1}$ vs. $\log (\mathrm{H} \alpha \mathrm{EW})$ plane. In both panels ages of the stellar population increase along the tracks from $100 \mathrm{Myr}$ on the upper left to $13 \mathrm{Gyr}$ on the lower right. Also in both panels the upper set of tracks have $\Gamma=1.00$ while the lower set have $\Gamma=2.00$. The arrows are dust vectors are for typical observed Balmer decrements $(\mathrm{H} \alpha / \mathrm{H} \beta=4)$ for different values of $f$. The values of $f$ are 1,2 and 4 clockwise from the one pointing left. (a) The effect of smooth SFH variation is shown in the left panel. The solid lines have exponentially decreasing SFRs with $\tau=1.1 \mathrm{Gyr}$, the dotted lines have more slowly falling SFRs with $\tau=2.75$ Gyr and the dashed lines are increasing $\propto 1-e^{-t / \tau}$ where $\tau=1.5 \mathrm{Gyr}$. These tracks have solar metallicity. (b) The effects of metallicity at fixed SFH with $\tau=1.1 \mathrm{Gyr} . Z=0.005$ for the dashed line, 0.010 for the dotted line, 0.020 for the solid line and 0.025 for the dot-dashed line. The solid lines are identical across the panels. This figure demonstrates that the effects of model parameters are largely orthogonal to IMF variations. 


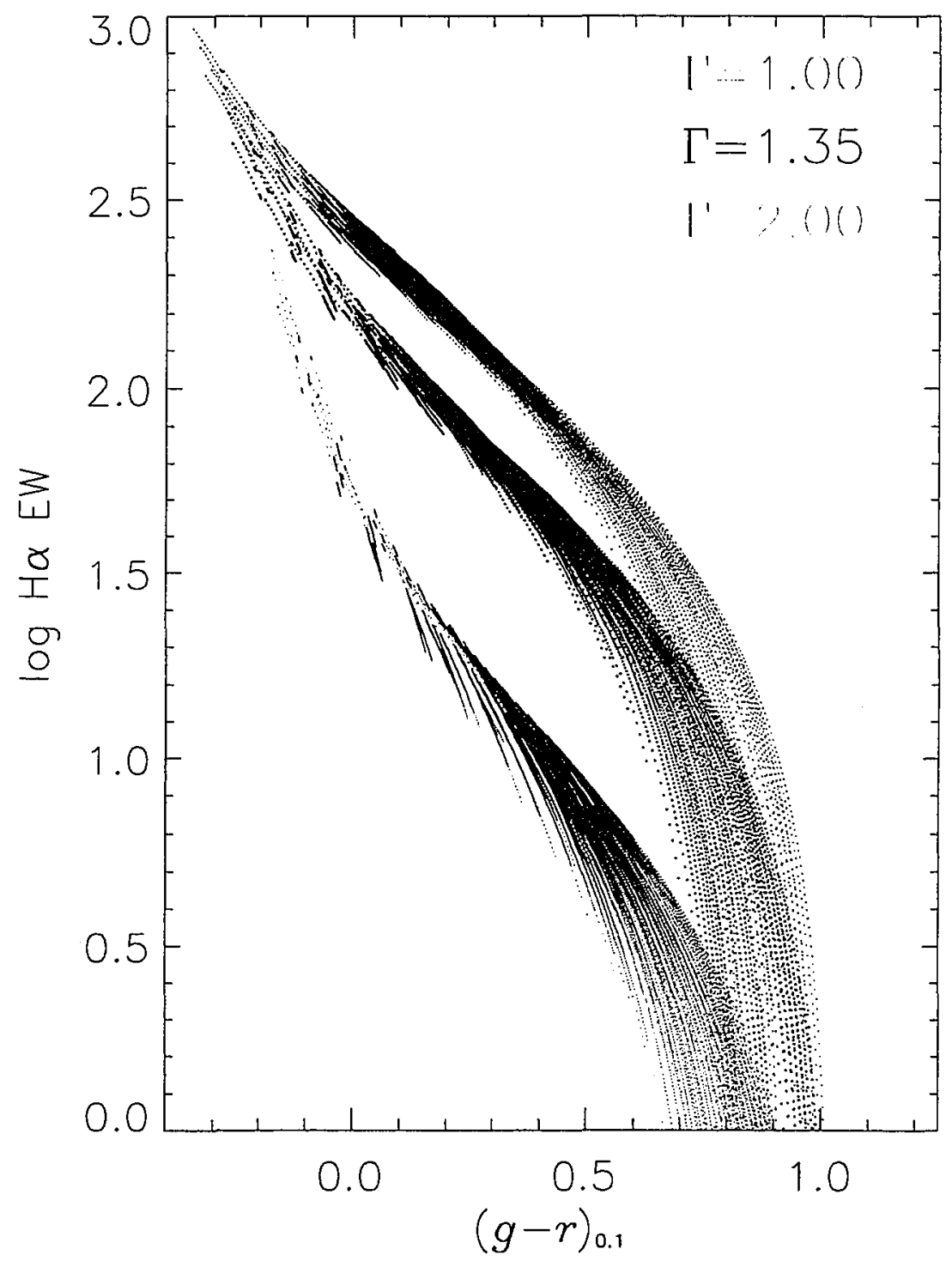

Figure 2.9 All model points for $\Gamma=1.00$ (cyan), $\Gamma=1.35$ (black) and $\Gamma=2.00$ (magenta). Each IMF has 18,480 calculated model values. $Z$ ranges from 0.005 to 0.025 , ages range from $100 \mathrm{Myr}$ to $13 \mathrm{Gyr}$ and SFHs cover the range described in the text. 


\section{Chapter 3}

\section{Evidence for a Non-Universal Stellar Initial Mass Function}

\subsection{Monte Carlo Results}

Figure 3.1 shows the results of our analysis for the full sample of galaxies using just the observed data. set. The "X" marks the best fitting IMF, where $\Gamma=1.4411$ and $\chi^{2}=60549.7$ with $\overline{\chi^{2}}=0.46$. At $\Gamma=1.00 \overline{\chi^{2}}=3.61$ while steeper IMFs are more heavily rejected with $\overline{\chi^{2}}=9.00$ at $\Gamma=2.00$.

For comparison several "classic" IMFs are also plotted in figure 3.1 at their approximate equivalent values of $\Gamma$. With $\overline{\chi^{2}}=7.73$ the Miller \& Scalo (1979) solar neighborhood IMF is a particularly bad fit. Two more recent solar neighborhood IMFs, Scalo (1986) and Kroupa, Tout, \& Gilmore (1993), yield $\overline{\chi^{2}}=2.38$ and 1.98 respectively. These results reinforce the conclusions of K83, KTC94 and Baldry \& Glazebrook (2003) that the solar neighborhood is not representative of galaxies on the whole as far as the IMF is concerned.

On the other hand the Scalo (1998) IMF, established from a review of star cluster 
IMF studies in the literature, is a better fit than the best, $\Gamma$ value in our parameterization with $\overline{\chi^{2}}=0.43$. This result highlights the degeneracy of the IMF models themselves in the color-H $\alpha$ EW plane-- two considerably different IMFs (one with one break and the other with two) fit nearly equally as woll.

The results of the MC simulation show that the $95 \%$ confidence region is $1.4432<$ $\Gamma<1.4443$ for the data set as a whole. The MC simulation shows that additional data will not improve the overall results as the random errors are already small. Clearly and not surprisingly systematic errors, which are discussed later, dominate.

The result for the sample as a whole is $\Gamma=1.4437 \pm 0.0005$. The error quoted here is the random error. This is steeper than the original Salpeter value of $\Gamma=1.35$. It is also steeper than the Baldry \& Glazebrook (2003) value of $\Gamma=1.15 \pm 0.2$ derived from galaxy luminosity densities in the UV to NIR. It is however well within their $95 \%$ confidence limit of $\Gamma<1.7$ as well as their measurement of $\Gamma=1.2 \pm 0.3$ based on the $\mathrm{H} \alpha$ luminosity density. The difference between their two results suggests that the $\mathrm{H} \alpha$ and mid-UV to optical fluxes may have different sensitivities to massive stars. Scalo (1998) estimated the uncertainty in $\Gamma$, either due to measurement uncertainties, real IMF variations or both, in his star cluster IMF based on the spread of results in the literature. Our result is well within his range of uncertainty in both mass regimes- $\Gamma=1.7 \pm 0.5$ for $1-10 \mathrm{M}_{\odot}$ and $\Gamma=1.3 \pm 0.5$ for $1-100 \mathrm{M}_{\odot}$

\subsubsection{Luminosity Effects}

The luminosity of a galaxy could potentially have an effect on the IMF within it. For one the ambient radiation field is likely higher in more luminous galaxies. Figure 3.2 
shows the best fitting IMF and $\overline{\chi^{2}}$ values as a function of $M_{r, 0.1}$ for all 130,602 galaxies. The galaxies have been binned in $\mathrm{M}_{r, 0.1}$ such that there are 500 objects in each bin. The bin size was chosen to maximize coverage in $\mathrm{M}_{r, 0.1}$ yet still keep the random errors in each bin small. The solid lines represent the lower and upper $95 \%$ confidence region determined from the MC simulation.

Figure 3.2 reveals a constant value of $\Gamma \sim 1.4$ for galaxies with $\mathrm{M}_{r, 0.1}$ between -21 and -22 with linear increases in $\Gamma$ for both brighter and fainter galaxies. There is also a sudden downturn in $\Gamma$ values for galaxies fainter than $\mathrm{M}_{r, 0.1}=-16.5$. Given the sizes of the random errors the differences in $\Gamma$ between $M_{r, 0.1}=-17$ and -22 are substantial, from 1.59 to 1.41 , and statistically significant. The agreement with the Salpeter slope is the best for galaxies between -21 and -22 in $\mathrm{M}_{r, 0.1}$.

In many ways it is not surprising that previous investigators have not found this trend. The Milky Way is thought to have a luminosity of $M_{V}=-20.9$ (Delhaye 1965); the $V$ and $r_{0.1}$ filter curves cover roughly the same wavelengths. At comparable luminosities our results are similar to Salpeter. The galaxies in the K83 sample have a median $\mathrm{M}_{B}=-20.9$ with only $16 \%$ (18 objects) fainter than $M_{B}^{*}=-19.7$ (Efstathiou, Ellis, \& Peterson 1988). This is a significant bias toward more luminous galaxies where our results are in agreement with a universal IMF. By contrast $30 \%$ of our sample is fainter than $M_{r, 0.1}^{*}=-20.44$ (Blanton, et al. 2003b). We have a sample of 39,350 galaxies fainter than $L^{*}$.

The lower panel of Figure 3.2 shows that the relative quality of the fits rapidly deteriorates as the luminosity of the galaxies decrease. For the brightest galaxies $\overline{\chi^{2}}$ floats around 0.15 , while in the faintest bin it is over 6 . For comparison $M_{V}=-18.5$ for the Large 
Magellanic Cloud and -17.1 for the Small Magellanic Cloud (Courtean \& van den Bergh 1999). This trend could indicate that a universal IMF is a good fit to the most luminous galaxies, but dwarf galaxies cannot be described by a universal IMF, even if a different universal slope is allowed. However it could have a more mundane explanation. It could be that errors are over or underestimated as a function of luminosity. It also could arise from deviations from our assumption of smoothly varying SFHs.

We cannot bin our data by stellar mass without assuming an IMF which is contrary to the goals of the project. We can repeat the analysis of Figure 3.2 using $M_{z, 0.1}$ in the place of $\mathrm{M}_{r, 0.1} \cdot \mathrm{M}_{z, 0.1}$, being redder, is a better proxy to stellar mass. The resulting plot, Figure 3.3 , is nearly identical to Figure 3.2 which shows that the relationship persists across several wavebands.

Figure 3.2 reveals a clear, statistically significant trend in $\Gamma$ and $\overline{\chi^{2}}$ with respect to luminosity. The rest of this section focuses whether this trend is a manifestation of true IMF variations or if it is the result of sample biases or poor assumptions.

\subsubsection{Sources of Bias}

If the IMF is truly universal and our method successfully probes the IMF any subsample of galaxies that we could choose should yield the same $\Gamma$ value as any other in spite of any selection biases or aperture effects. Figure 3.2 clearly shows that the preceding statement is false. In this section we set aside the possibility of IMF variations and scarch for biases in our sample. 


\section{Magnitude Limited Sample}

Figure 3.2 shows that the overall result of $\Gamma=1.4437$ is really a weighted average. The SDSS MGS is a magnitude limited sample, one defined by flux limits, with both upper and lower limits. Table 3.1 gives the number of objects in each luminosity bin. There are 41,411 galaxies with $-21.5<\mathrm{M}_{r ; 0.1}<-20.5$ but only 28 for which $-14.5<\mathrm{M}_{r, 0.1}<-13.5$. As such the overall result is heavily biased by more luminous galaxies.

Malmquist bias will affect any magnitude limited sample. Because brighter objects can be seen at greater distances a magnitude limited sample contains bright objects from a greater volume of space than fainter objects. The result is that the ratio objects by luminosity in a magnitude limited sample differs from the true ratio in nature; brighter objects are over-represented.

To eliminate Malmquist bias volume limited bins where subsamples are complete for a range of luminosities are constructed. Figure 3.4 details the construction of these bins. Given both the upper and lower flux limits of the MGS $(15.0<r<17.77)$ only a factor of 13 in luminosity falls in the sample at any given redshift. The redshift limits of each volume limited bin are defined such that no galaxies within the magnitude limits of the bin are affected by the flux limits of the MGS. Within each box in Figure 3.4 the true ratio of galaxy luminosities is preserved and is thus free of Malmquist bias.

Figure 3.5 shows the results for volume limited magnitude bins. Most error bars are smaller than the plotting symbols due to the larger number of galaxies, 329 to 29,701 as given in Table 3.1 , in each bin. Figures 3.2 and 3.5 show the exact same trends. The largest difference in $\Gamma$ between the whole and volume limited samples is 0.0116 in the 
Table 3.1. Luminosity Bin Details

\begin{tabular}{|c|c|c|c|c|c|c|c|c|c|c|c|}
\hline \multirow[b]{2}{*}{$M_{r, 0.1}$} & \multirow[b]{2}{*}{$n$} & \multirow[b]{2}{*}{$\Gamma_{\text {low }}$} & \multicolumn{3}{|c|}{ Volume Limited } & \multirow[b]{2}{*}{$\bar{z}$} & \multirow[b]{2}{*}{$\overline{A_{V, l}}$} & \multirow[b]{2}{*}{$\overline{\mathrm{ap}}$} & \multicolumn{3}{|c|}{ All } \\
\hline & & & $\Gamma_{\text {best }}$ & $\Gamma_{\text {high }}$ & $\overline{x^{2}}$ & & & & $n$ & $\Gamma_{\text {best }}$ & $\overline{x^{2}}$ \\
\hline-14 & - & - & - & - & - & - & - & - & 28 & 1.3835 & 7.43 \\
\hline-15 & - & - & - & - & - & - & - & - & 188 & 1.3892 & 9.86 \\
\hline-16 & - & - & - & - & - & - & - & - & 406 & 1.5461 & 4.64 \\
\hline-17 & 329 & 1.6000 & 1.6045 & 1.6086 & 3.41 & 0.013 & 0.38 & 0.20 & 1,304 & 1.5879 & 2.57 \\
\hline-18 & 1,555 & 1.5338 & 1.5370 & 1.5424 & 2.05 & 0.022 & 0.50 & 0.21 & 4,327 & 1.5326 & 1.57 \\
\hline-19 & 4,935 & 1.4772 & 1.4788 & 1.4813 & 1.17 & 0.032 & 0.70 & 0.21 & 10,375 & 1.4813 & 0.96 \\
\hline-20 & 12,951 & 1.4306 & 1.4320 & 1.4330 & 0.63 & 0.050 & 0.91 & 0.22 & 24,851 & 1.4436 & 0.56 \\
\hline-21 & 28,633 & 1.4051 & 1.4057 & 1.4063 & 0.32 & 0.077 & 1.15 & 0.24 & 41,411 & 1.4064 & 0.31 \\
\hline-22 & 29,701 & 1.4036 & 1.4042 & 1.4050 & 0.19 & 0.116 & 1.32 & 0.25 & 38,406 & 1.4084 & 0.20 \\
\hline-23 & 8,049 & 1.4545 & 1.4556 & 1.4568 & 0.15 & 0.168 & 1.55 & 0.27 & 9,106 & 1.4505 & 0.16 \\
\hline-24 & - & - & - & - & - & - & - & - & 192 & 1.5329 & 0.12 \\
\hline All* & 130,602 & 1.4432 & 1.4437 & 1.4443 & 0.50 & 0.090 & 1.05 & 0.25 & - & - & - \\
\hline
\end{tabular}

Note. - Propertics of the $\mathrm{M}_{r, 0.1}$ luminosity bins. Columns 2 through 9 give values for volume limited magnitude bins while columns 10,11 and 12 give values for all sample galaxies within the luminosity range. $\overline{a p}$ is the mean aperture fraction. * The sample as a whole is not volume limited but the more detailed information is given for reference. 
$\mathrm{M}_{r, 0.1}=-17 \mathrm{bin}$. The other notable difference is that the fainter galaxies have larger $\overline{\chi^{2}}$ values in the volume limited case. However Malmquist bias across bins is not responsible for the luminosity trends in Figure 3.2.

\section{Redshift}

Another effect of magnitude limited samples is that the faintest galaxies are much closer than the most luminous ones. The mean redshifts of the volume limited magnitude bins range from $z=0.013$ for $\mathrm{M}_{r, 0,1}=-17$ to $z=0.168$ for $\mathrm{M}_{r, 0.1}=-23$. This corresponds to a difference in age of around $1.8 \mathrm{Gyr}$. As aforementioned, model tracks reveal that age is largely orthogonal to the IMF in our parameter space, but there could be other effects tied to age and distance. In addition the IMF could evolve with time.

The large number of galaxies in our sample affords us the luxury of investigating the effects of luminosity and redshift simultaneously to obtain a better understanding of what role, if any, the redshift plays in our analysis. Figure 3.6 shows our fitted parameters for all 130,602 galaxies in bins that are 0.25 magnitudes wide in luminosity and 0.005 wide in redshift. The upper left panel shows the best fitting $\Gamma$ for each two dimensional bin. On the upper right the width of the $95 \%$ confidence region in $\Gamma$ for each bin is shown. At bottom left is the $\log \overline{\chi^{2}}$ and at bottom right is the log of the number of galaxies in each bin. The white contour demarcates the region in which each bin contains at least 50 galaxies. The number 50 is arhitrary hut it shows the region where Poisson errors are expected to be small. The black areas are regions where there are no galaxies with the given parameters.

Using the plot of $\Gamma$ at the upper left we can look for potential redshift biases. This is complicated by the fact that at a fixed luminosity there is a limit to the range 
of redshifts in the sample due to the flux limits of the sample described earlier. Looking at vertical slices through the plot at any fixed luminosity there is a trend towards larger values of $\Gamma$ with increasing redshift. However, for horizontal slices of fixed redshift the same relationship between $\Gamma$ and luminosity that is present for the whole sample is seen modulo a normalization factor.

The right half of Figure 3.6 shows a strong relationship between the number of galaxies per bin and the width of the $95 \%$ confidence region in $\Gamma$. This simply reflects the fact that larger samples are less affected by Poisson errors.

The lower left panel of Figure 3.6 provides an excellent example of why our metric of fit quality, $\overline{\chi^{2}}$, is so important. Bins with similar numbers of galaxies and $\delta \Gamma$ values can have vastly different values of $\overline{\chi^{2}}$. It is worth reminding that the contours in $\overline{\chi^{2}}$ are logarithmic. At fixed luminosity the galaxies are better fit by a universal IMF at higher redshift. Similar to the sample as a whole the quality of fit improves with luminosity.

While there does appear to be some weak trending of $\Gamma$ and $\overline{\chi^{2}}$ with redshift, redshift effects are not driving the relationship seen between IMF and luminosity as it persists at fixed redshifts.

\section{Aperture Effects}

One explanation for the trend in $\Gamma$ with redshift is aperture effects. Again, if the TMF is truly universal aperture effects should not exist. The SDSS spectra have a fixed aperture of 3" for all galaxies. Depending on the angular extent and distance to a galaxy a different fraction of the total light of the galaxy will fall into the aperture. The problem is mitigated by the fact that the most distant galaxies are the most luminous and 
more likely to have a larger physical size. As the physical area contained in the aperture increases with distance, so too does the size of the galaxies being observed. However, the two effects do not exactly balance out. Table 3.1 shows that the mean aperture fraction for the $\mathrm{M}_{r, 0.1}=-17$ bin is 0.20 and increases to 0.27 at $\mathrm{M}_{r, 0.1}=-23$. On average $35 \%$ more of the most luminous galaxies fall within the aperture compared to the faintest.

Figure 3.7 shows the behavior of our fitted parameters for two dimensional bins of luminosity and aperture fraction in the same manner as Figure 3.6 did for luminosity and redshift. For fixed luminosities increasing aperture fraction leads to decreasing values of $\Gamma$. However at fixed aperture fraction the qualitative IMF-luminosity trend remains. The $\overline{\chi^{2}}$ values are a strong function of luminosity, but $\overline{\chi^{2}}$ does increase with aperture fraction at each fixed luminosity.

The trend with aperture fraction is the exact opposite of what would be expected in the presence of a systematic effect operating given the redshift result in Figure 3.6. The nearest galaxies should have the smallest aperture fractions in a particular luminosity bin. The nearest galaxies in Figure 3.6 have the smallest values of $\Gamma$ while the smallest aperture fractions in Figure 3.7 have the largest values of $\Gamma$.

Figure 3.7 suggests that the measured IMF is more dependent on the aperture fraction than the redshift. There are several possible physical explanations for IMF trends with the aperture fraction, all of which are related to radial gradients in disk galaxies. Paduan, Nordlund \& Jones (1997) make the theory based claim that the IMF should be a function of the original local temperature of the star-forming molecular clouds. Metallicity gradients are also known to exist in disk galaxies, including the Milky Way (Mayor 1976). 
Rolleston, et al. (2000) measure a linear, radial light metal (C, O, Mg \& Si) abundance gradient of $-0.07 \pm 0.01 \mathrm{dex} \mathrm{kpc}^{-1}$ in the disk of the Milky Way. Given the increased efficiency of cooling with metal lines we would expect the most low mass stars where metallicity is the highest- on average towards the center of galaxies. The trend in $\Gamma$ in Figure 3.7 is qualitatively consistent with this idea.

If there are radial IMF gradients in galaxies one would expect the fits to decrease in quality with increasing aperture fraction. A blend of IMFs will not be fit as well as a universal one given our technique. This idea is consistent with the results in Figure 3.7. However, in well-resolved stellar populations there is no evidence for a relationship between the IMF and metallicity, except perhaps at masses lower than those probed by our method (Kroupa 2002). If metallicity plays a role in determining the IMF the effects are only being revealed as a global trend in our large sample of integrated stellar populations. For individual clusters metallicity must play a secondary role to stochastic effects.

\section{Extinction Correction}

The extinction correction is another potential source of bias. It is possible that there is a second order correction that our fairly simple extinction correction fails to take into account. This could potentially lead to an erroneous IMF trend with extinction correction. This affects the luminosity results because more luminous galaxies tend to be dustier, as evidenced in Table 3.1. The problem is further complicated by the fact that dust is thought: to play an integral part in star formation so it is not unreasonable that an observed IMF trend with extinction may be real.

Figure 3.8, similar to Figures 3.6 and 3.7 , shows the results of our analysis for 
two dimensional bins of luminosity and the extinction correction that was measured and applied. Vertical slices through the upper left panel of Figure 3.8 show that $\Gamma$ does depend on $A_{V, l}$, trending towards lower $\Gamma$ values with increased extinction over the region where the Poisson error in $\Gamma$ is reasonable. Yet again, horizontal cuts of fixed extinction show the IMF-luminosity relationship.

The decreasing $\Gamma$ values with increasing extinction is counter-intuitive. Dustier regions tend to be more metal rich. If metal cooling plays a significant role in the IMF the dustiest regions should have the steepest IMFs.

At fixed luminosity $\overline{\chi^{2}}$ increases with extinction. As aforementioned our calculated errors in color and EW (equations 2.4 and 2.6) have a functional dependence on the observed emission line extinction. In both cases it is the term proportional to $\sigma_{f} A_{V, l}$ which is on average the major contributor to the calculated error. Because luminous galaxies tend to be more heavily extincted they will also be more likely to have larger errors. This is potentially problematic for our observed IMF trend with luminosity. If we are unknowingly underestimating the errors for faint galaxies with low extinction the source of the poor fit qualities of these galaxies could be systematic instead of astrophysical. However the lower left panel of Figure 3.8 shows that the most extincted galaxies have the poorest fits where such a bias would suggest that they should fit the best due to the large accommodating errors.

\section{Multiple Parameter Biases}

It is also possible that biases in our $\Gamma$ measurements could depend on two parameters simultaneously. Figure 3.9 shows the measured galaxy of $\Gamma$ as a function of both 
aperture fraction and measured emission line extinction for six volume limited luminosity bins.

When holding all other parameters fixed, increasing the aperture fraction leads to lower values of $\Gamma$ in all statistically significant areas of Figure 3.9. This is the same relationship found in the earlier section on aperture fraction. Decreasing values of $\Gamma$ are also seen for increasing extinction when all other parameters are constant. A notable exception to this is that galaxies with large extinction and small aperture fractions favor higher $\Gamma$ values.

Most importantly when looking at a particular combination of aperture fraction and extinction the IMF becomes shallower with increasing luminosity until the highest luminosities where it becomes steeper again. Even in the narrowest slices of the data set the same IMF-luminosity trend is seen, albeit with slightly different absolute values of $\Gamma$.

\section{Star Formation Strength}

As discussed previously we have allowed two classes of star forming galaxies into our sample. 111,806 galaxies (86\%) fall in the star forming class and the other $18,796(14 \%)$ belong to the low S/N star forming class where the O III or $\mathrm{N}$ II lines are weak, but the $\mathrm{H} \alpha$ and $\mathrm{H} \beta$ lines still have $\mathrm{S} / \mathrm{N}>5$. By comparing the results from these two subsamples we can investigate a possible bias of the results with respect to the level of star formation.

Figure 3.10 shows the results for both classes as a function of luminosity. $\Lambda$ s it comprises $86 \%$ of the total sample it is not surprising that the results for the star forming class are similar to those of the sample as a whole in Figure 3.2.

The low $\mathrm{S} / \mathrm{N}$ class exhibits a similar qualitative behavior to the set as a whole 
with a few notable differences. The $\Gamma$ values are offset by at least 0.08 towards larger $\Gamma$. The measured IMF turns toward steeper values at lower luminosities than for the sample as a whole. The $\overline{\chi^{2}}$ are several times lower as well.

While the galaxies in low $\mathrm{S} / \mathrm{N}$ class meet the same requirement of $\mathrm{S} / \mathrm{N}>5$ in the Balmer lines as the star forming class they are biased towards noisier Ha line measurements. This corresponds to lines that are either weak (low SFR) or weak compared to the continuum (low present SFR compared to the past) both of which lead to low EWs.

Another issue at play is that the relationship between the $\mathrm{H} \alpha$ line flux and the measured SFR is dependent on the IMF and to a lesser extent the metallicity. This effect is shown in Figure 3.11. The figure shows the $\mathrm{H} \alpha$ flux as a function of $\Gamma$ for four different metallicities. All models in Figure 3.11 have the same, constant underlying SFR. At a fixed metallicity and SFR increasing $\Gamma$ by 0.05 reduces the $\mathrm{H} \alpha$ flux by $20 \%$. In fact the $\mathrm{H} \alpha$ flux of a galaxy with $\Gamma=1.00$ will be 33 times larger than a galaxy with the same SFR and $\Gamma=2.00$ at solar metallicity. The effect is even stronger at lower metallicities. In the presence of real IMF variations at any fixed luminosity the low $\mathrm{S} / \mathrm{N}$ class will be biased towards galaxies with steeper IMFs.

Both low SFRs and steep IMFs potentially lead to low $\mathrm{S} / \mathrm{N} \mathrm{H} \alpha$ flux. However it is determine the level of influence of each effect. The conversion from the $\mathrm{H} \alpha$ flux to SFR. is usually a simple linear conversion (e.g. K83) based on case B recombination as described earlier. As a result Figure 3.11 also shows the relative uncertainty of the SFR measured from the $\mathrm{H} \alpha$ flux. Therefore an increase of 0.05 in $\Gamma$ will also lead to underestimating the true SFR by $20 \%$. The relationships shown in Figure 3.11 are for a constant SFH 
and undoubtedly become more complicated when a range of SFHs are considered. For a universal IMF the results of Figure 3.10 could be interpreted as being the result of a physical link between the IMF and the SFR. However Figure 3.10 suggests that the IMF is not universal. This suggests that the offset is due to a complicated mixture of IMF and SFR rate effects which cannot be decoupled at this time.

As shown in Figure 2.1 low $\mathrm{H} \alpha$ EWs lead to larger values of $\Gamma$ for any fixed color. As the low $\mathrm{S} / \mathrm{N}$ class tends toward noisier $\mathrm{H} \alpha$ fluxes and therefore EWs it is easy to see from equations $2.4,2.5$ and 2.6 that the errors for this class will tend to be larger. This in turn leads to lower $\chi^{2}$ values.

As the qualitative IMF-luminosity trend occurs in both star forming classes the strength of star formation is unlikely to be a significant bias on our results.

\section{The $f$ Ratio}

As mentioned in the data corrections section, the $f$ ratio is ratio of the extinction experienced by the nebular emission lines to that experienced by the stellar continuum. The assumption of a value for $f$ could potentially bias our results. An alternative way of looking at the same problem is that our data in the color- $\mathrm{H} \alpha \mathrm{EW}$ plane can be used to constrain the $f$ ratio by assuming a universal Salpeter IMF.

Figure 3.12 gives the results of this analysis for the data set as a whole. A value of $f=2.0$ is found with $\overline{\chi^{2}}=1.068$. This is in good agreement with to the Callact.i, Kinney, $\&$ Storchi-Bergmann (1994) value of $f=2.0+0.6 /-0.4$. The quality of the fit in the best case is worse than in Figure 3.1. Part of the reason for this is that the errors used were slightly smaller as the $\sigma_{f}$ terms in equations 2.4 and 2.6 are set equal to 0 . The quality 
of the fit drops sharply below $f=2$ and more gradually for $f>2$. Values of $f$ near 1 are heavily rejected. However in this particular plot the results are dominated by luminous galaxies.

The values of $f$ as a function of luminosity are shown in Figure 3.13. For galaxies $\mathrm{M}_{r, 0.1}=-19$ and brighter the best value of $f$ is consistent with $f=2$. The faintest two bins the prefer an $f$ ratio closer to 2.5 . However the lower panel shows that this new $f$ value does not translate to improved fit quality. In fact the faintest galaxies have in general smaller measured extinctions and are therefore less susceptible to changes in $f$. The same qualitative trend of worsening fits with decreasing luminosity seen when allowing $\Gamma$ to float is seen with a varying $f$ value.

Together these two $f$ ratio plots provide a number of insights. For one it shows that our choice of the $f$ ratio is very sensible and provides an independent confirmation of other $f$ ratio measurements. The fact that our best fitting $\Gamma$ values are at least 0.05 above the Salpeter value cannot be reconciled by changing the geometry of the dust screen. It provides further evidence that the relationship between $\Gamma$ and luminosity is not a function of extinction or a byproduct of our extinction correction.

\section{Summary}

In this section we have investigated several possible sources of bias to account for our observed trend between the IMF and lmminosity. Relationships between the IMF and redshift, aperture fraction, extinction and star formation strength have been uncovered. Two parameter biases were also found.

In all cases in narrow slices through the data where potential biases are held fixed 
the qualitative IMF-luminosity relationship appears. The parameters primarily act to offset the value of $\Gamma$ at a particular luminosity. The ratio of continuum to emission line extinction, $f$, was found to be a sensible choice and the results are not sensitive to small changes in this value.

There are two possible interpretations to the relationships between the IMF and potential biases. One is that they are systematic effects due to some problem with our measurement of $\Gamma$. The second is that they are real physical effects. It is not clear from the data which of these statements is more correct.

\subsubsection{Star Formation History}

In the previous section several possible sources of bias were invesiigated, but none were able to account for our observed trend in $\Gamma$ with luminosity or the inability of a universal IMF to fit low luminosity galaxies. Figure 3.14 shows the distribution in color$\mathrm{H} \alpha$ EW space for the least and most luminous bins, $\mathrm{M}_{r, 0.1}=-17$ and -23 . From this figure it is apparent that the most luminous galaxies lie roughly parallel to the IMF tracks while the faintest galaxies are more perpendicular to the tracks. In the low luminosity bin there are galaxies which are simultaneously blue and have low EWs. These galaxies are not consistent with a universal IMF with $\Gamma=1.35$ and as mentioned before are not consistent with a universal IMF with a different slope. In addition the faintest galaxies have the lowest extinctions they are the least sensitive to dust and $f$ ratio issues. Before concluding that this is evidence for IMF variations we must first consider whether our model assumption of smooth SFHs is justified. 


\section{Effects of Star Formation Bursts}

The SFH of individual galaxies is the most problematic aspect of the K83 analysis. A sudden burst on top of a smoothly varying background will immediately increase the $\mathrm{H} \alpha$ EW. This is due to the formation of $\mathrm{O}$ and $\mathrm{B}$ stars which indirectly increase the $\mathrm{H} \alpha$ flux through processing of their ionizing photons. The new presence of $\mathrm{O}$ and $\mathrm{B}$ stars also makes the color of the galaxy bluer. Both of these effects are proportional to the size of the burst.

After the burst is over the $\mathrm{H} \alpha \mathrm{EW}$ is smaller and the colors are redder than they would be if the burst had not occurred. The $\mathrm{H} \alpha \mathrm{EW}$ drops because there is no longer an excess of $\mathrm{O}$ and $\mathrm{B}$ stars and their ionizing photons which reduces the $\mathrm{H} \alpha$ flux to pre-burst levels. However there is now an excess of red giants due to the less massive stars from the burst leaving the main sequence. This increases the continuum around the $\mathrm{H} \alpha$ line which further drops the EW in addition to making the galaxy colors redder. After enough time has elapsed after the burst the galaxy returns to the same position in the color- $\mathrm{H} \alpha \mathrm{EW}$ plane it would have occupied had no burst occurred, although it will have taken longer to get there.

Figure 3.15 gives one example of this cycle. A solar metallicity galaxy with $\Gamma=$ 1.35 and an exponentially decreasing SFH with $\tau=2.15$ Gyr experiences a burst of star formation at an age of $4.113 \mathrm{Gyr}$ which lasts $250 \mathrm{Myr}$ and forms $10 \%$ of the stellar mass. The black dots, spaced at $100 \mathrm{Myr}$ intervals, show that comparatively more time is spent below the nominal track than above it. The peak $\mathrm{H} \alpha \mathrm{EW}$ is reached just $5 \mathrm{Myr}$ after the start of the burst. If you happen to be observing the galaxy during the burst a shallower IMF will be measured (assuming a burst-free SFH), after the burst for I Gyr a steeper 
IMF will be measured and after that the effects of the burst largely disappear, although the galaxy will appear younger than it actually is.

Figure 3.16 gives the best fitting IMF slope from our analysis, which assumes no bursts, as a function of age for the galaxy in Figure 3.15. The jitter in the best fit $\Gamma$ values is due to the discrete nature of our model grid, and the fact that the model tracks for different, IMFs run together at large ages. Within a $300 \mathrm{Myr}$ period during and just after the burst the best fitting IMF slope is anywhere from $\Gamma=1.00$ to 1.95 . This shows that even if a universal IMF exists the SFH can mimic a huge range of IMF models. Roughly 1 Gyr after the burst the measured IMF is back to its true value. A galaxy with a bursty SFH viewed at a random time will be biased toward a steeper IMF than what is the true IMF. While one model is not an exhaustive study of the effects of the SFH on IMF measurements it does give a good sense of what issues arise.

To eliminate this uncertainty we investigated cutting the sample on SFH. In order to detect a relative lack or excess of present star formation it is necessary to measure both the present and past star formation rates or at least be able to compare the two in some way. The problem lies in the fact that conversions of observables into star formation rates assume an IMF to do so. Our aim is to measure the IMF so we cannot make strong a priori assumptions about it. Instead of biasing or results from the start we fit all galaxies and then try to determine the affect of SFHs on our conclusions. 


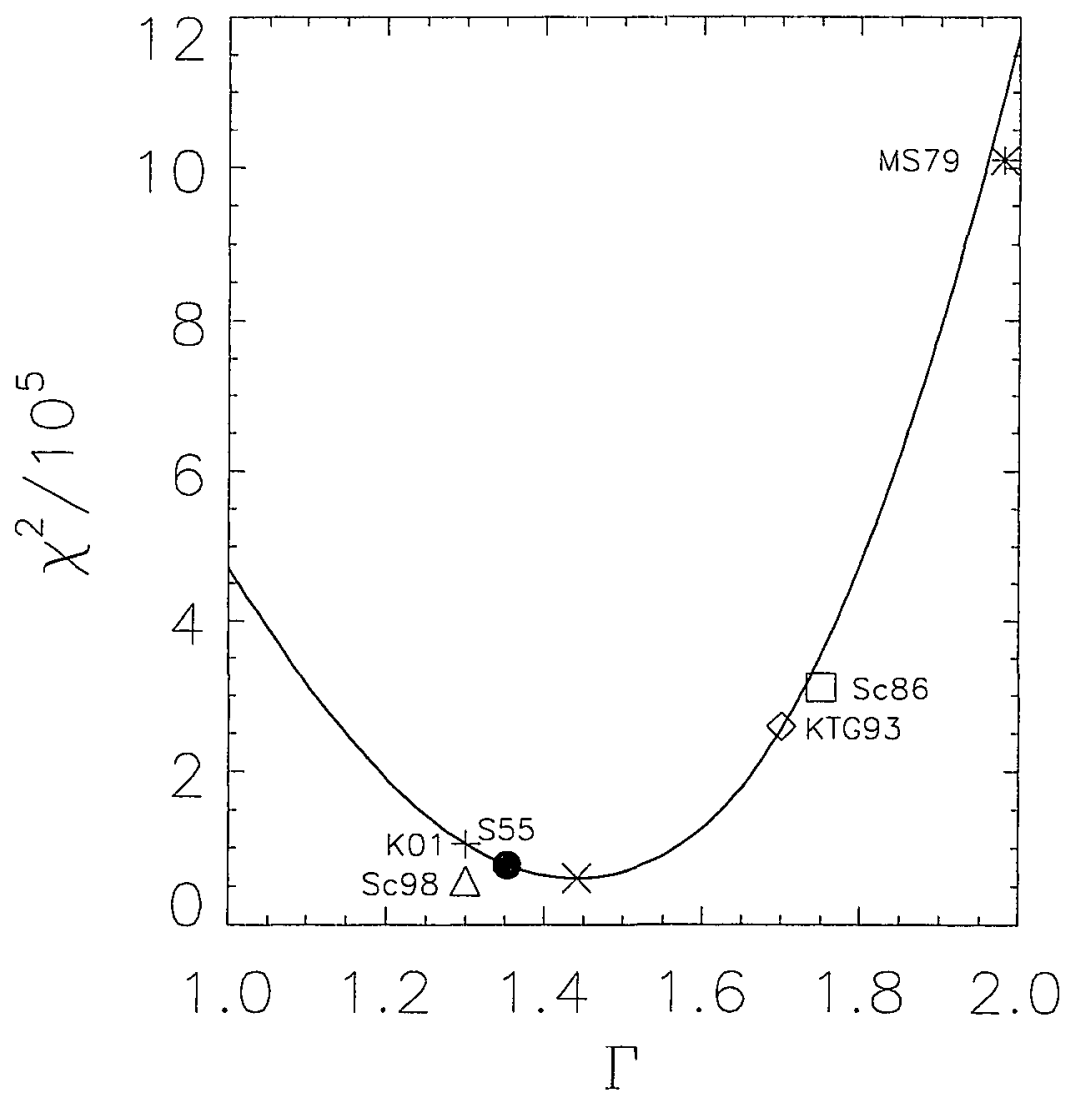

Figure 3.1 Value of $\chi^{2}$ as a function of $\Gamma$ for the entire sample (solid line). The " $\mathrm{X}$ " marks the minimum $\chi^{2}$ value of 61671.4 at $\Gamma=1.4525$. Also plotted are the $\chi^{2}$ for some "classic" IMF models, Salpeter (1955) (filled circle), Miller \& Scalo (1979) (asterisk), Scalo (1986) (square), Kroupa, Tout, \& Gilmore (1993) (diamond), Scalo (1998) (triangle) and Kroupa (2001) (plus sign) plotted at rough estimates for equivalent $\Gamma$ values using our parameterization. 


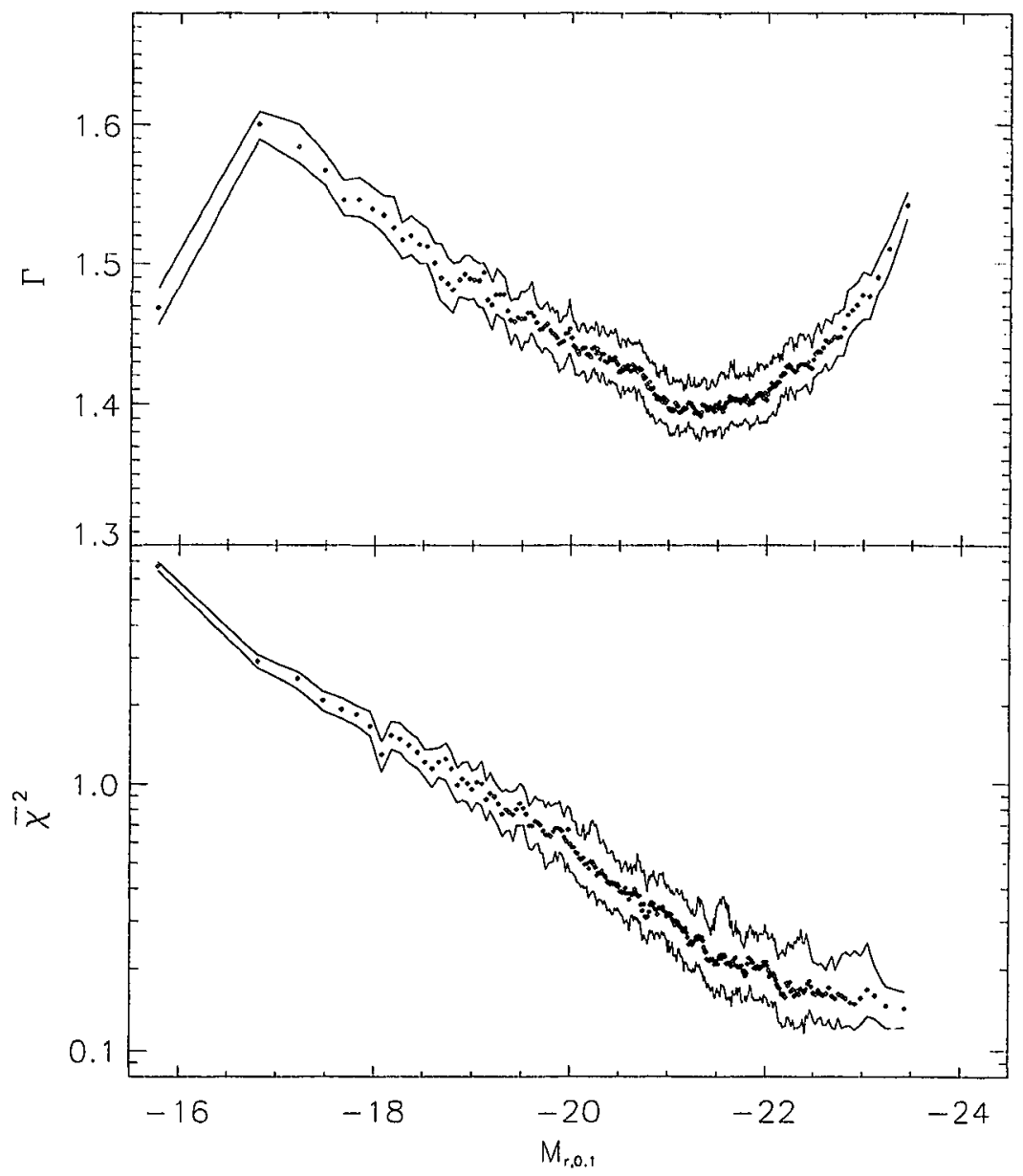

Figure 3.2 MC simulation results for the full sample binned by $M_{r, 0.1}$. Each diamond represents 500 galaxies plotted at the mean $\mathrm{M}_{r, 0.1}$ value of the bin. The points are the most dense around $\mathrm{M}_{r, 0.1}=-21.5$ as the sample is dominated by galaxies in this luminosity regime. The solid lines represent the upper and lower $95 \%$ confidence region measured for each bin. Top panel: The best fitting $\Gamma$ values as a function of $r$-band luminosity. Lower panel: The $\overline{\chi^{2}}$ values for each luminosity plotted on a log scale. 


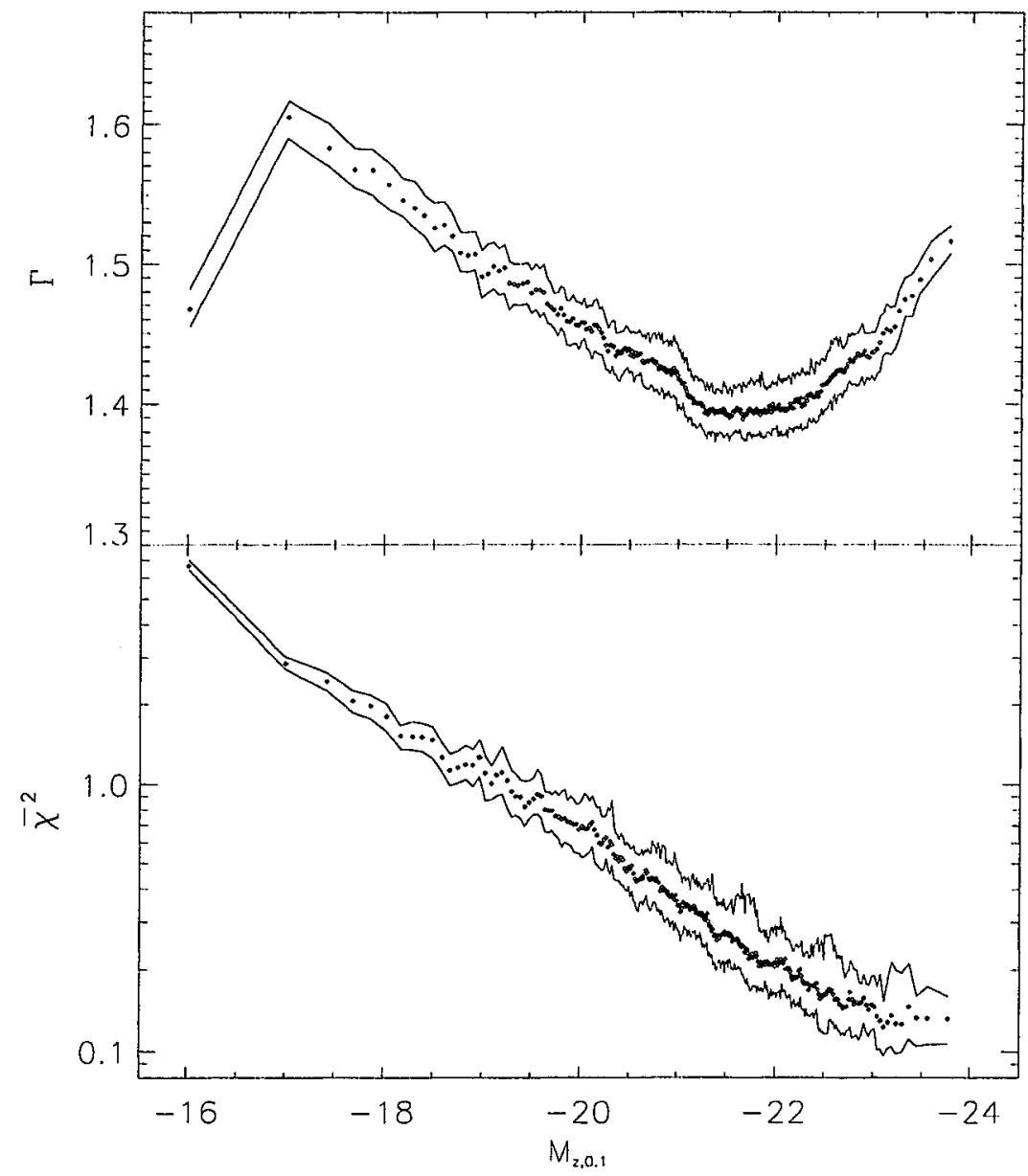

Figure 3.3 MC simulation results for the full sample binned by $\mathrm{M}_{z, 0.1}$. See the caption of Figure 3.2 for details. 


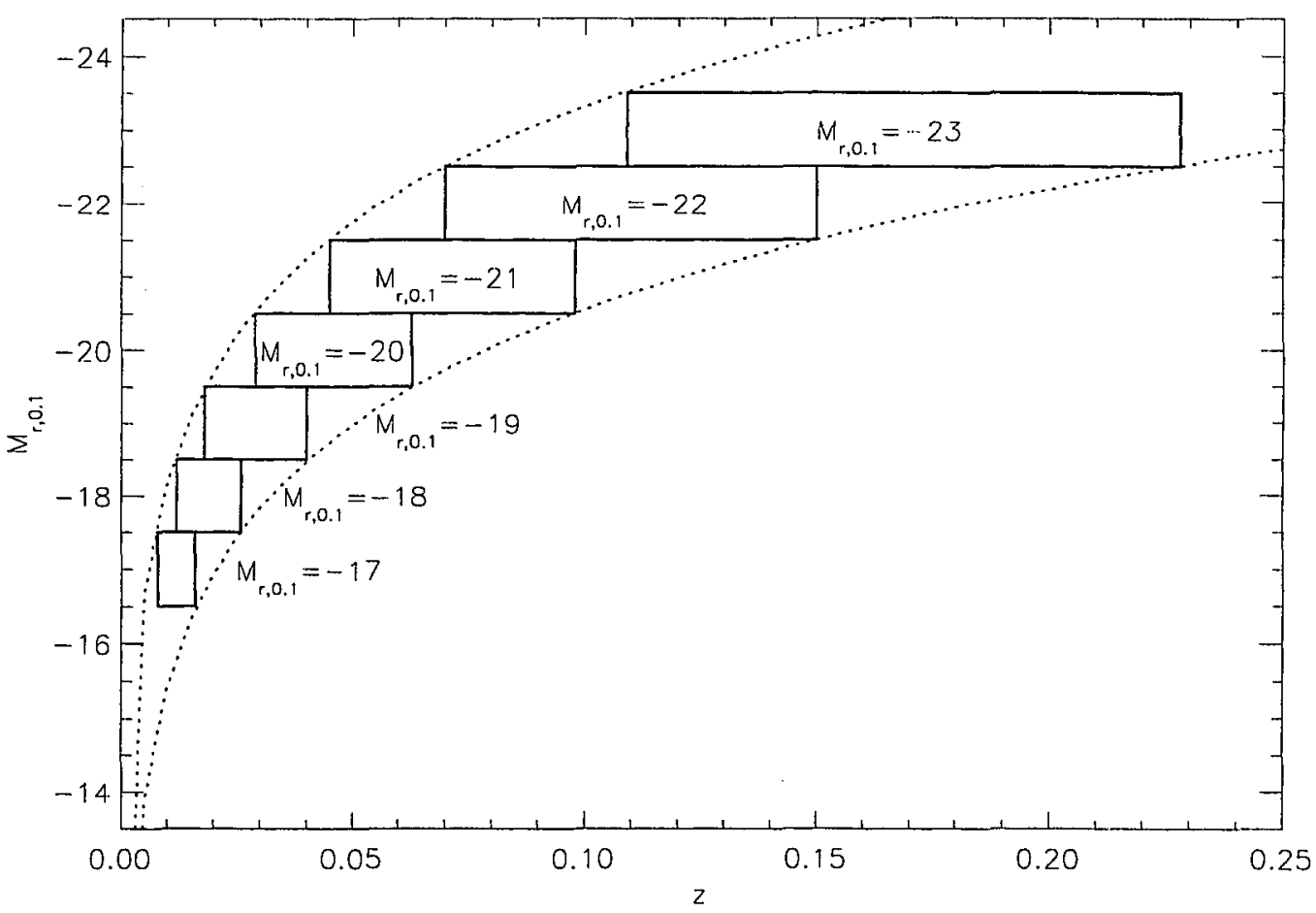

Figure 3.4 Definition of volume limited magnitude bins. The dotted lines show the luminosities corresponding to the flux limits of the SDSS MGS $(15.0<r<17.77)$ as a function of redshift. The solid boxes are the volume limited magnitude bins used in this paper. Within each box no galaxies within the magnitude range of the box are affected by the flux limits of the sample. 


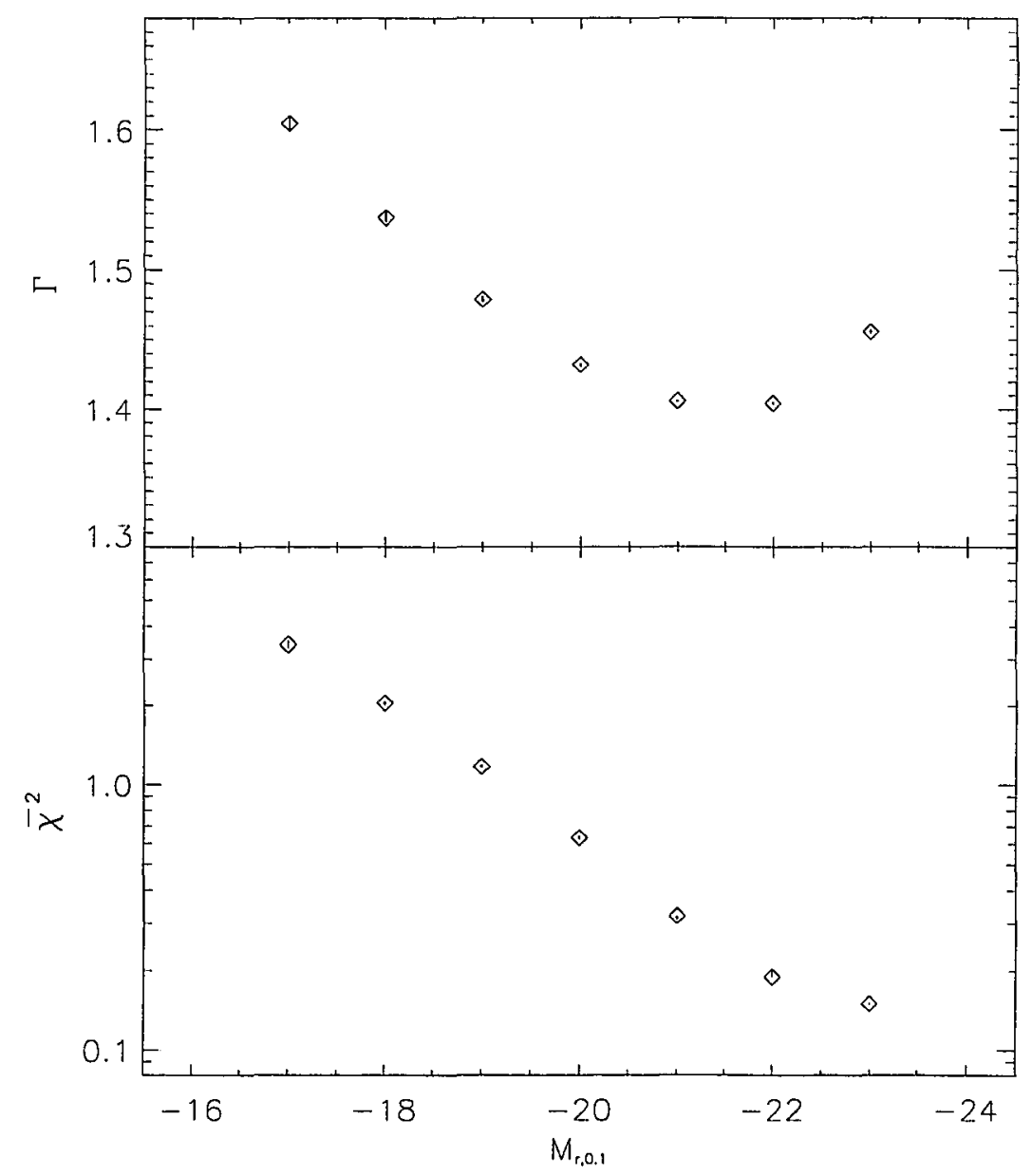

Figure 3.5 MC simulation results as a function of $\mathrm{M}_{r, 0.1}$ where the magnitude bins are volume limited. The error bars represent the $95 \%$ confidence interval from the simulation and are in most cases smaller than the plotting symbol. Top panel: The best fitting $\Gamma$ values as a function of $r$-band luminosity. Lower panel: The $\overline{\chi^{2}}$ values for each luminosity plotted on a log scale. 


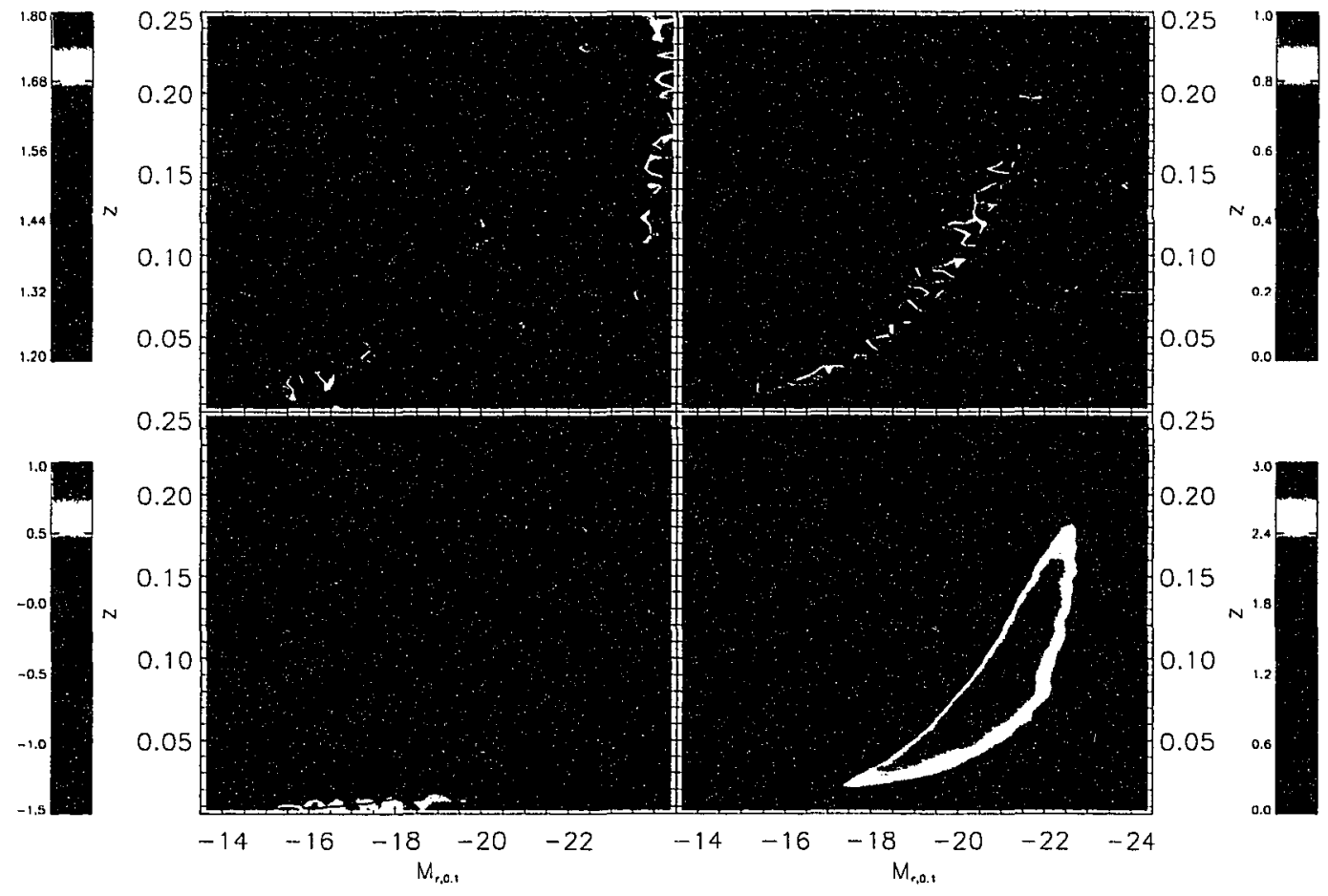

Figure 3.6 Values of fitted parameters as a function of both luminosity and redshift. The bins extend 0.25 magnitudes in luminosity and 0.005 in redshift. Clockwise from upper left the frames show the best fitting $\Gamma$, the width of the $95 \%$ confidence region in $\Gamma$ from the $\mathrm{MC}$ simulation, the $\log$ of the number of galaxies in each two dimensional bin and the $\log$ of $\overline{\chi^{2}}$. The shading levels for each panel are given by the adjacent vertical color bars. Black areas indicate regions where there are no galaxics with the respective combination of redshift and luminosity. The white contour indicates the region in which there are at least 50 galaxies in each 2-D bin. 


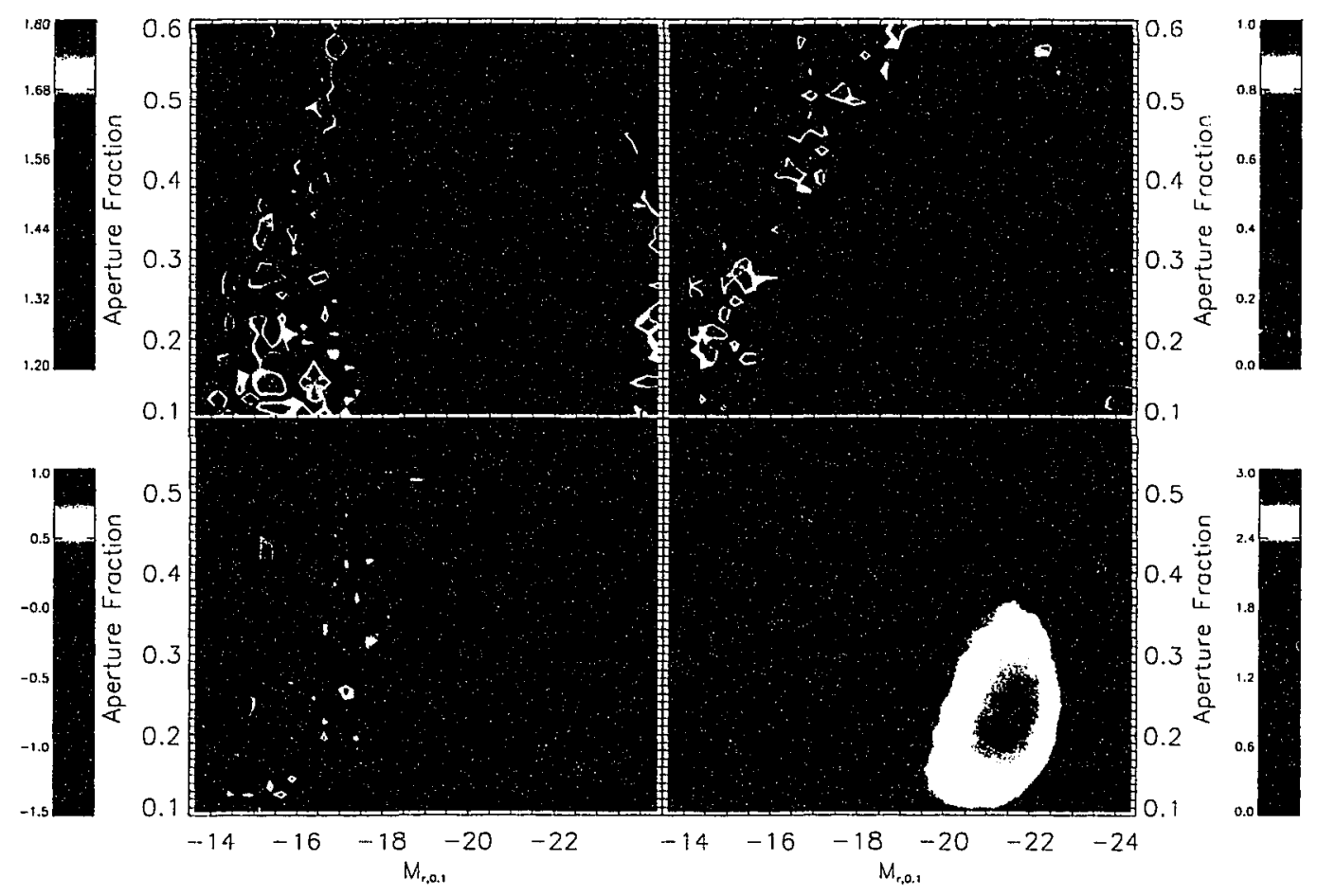

Figure 3.7 Values of fitted parameters for 2-D bins cut on luminosity and aperture fraction. The bins extend 0.25 magnitudes in luminosity and $1 \%$ in aperture fraction. The description is identical to Figure 3.6 as are the shading levels. 


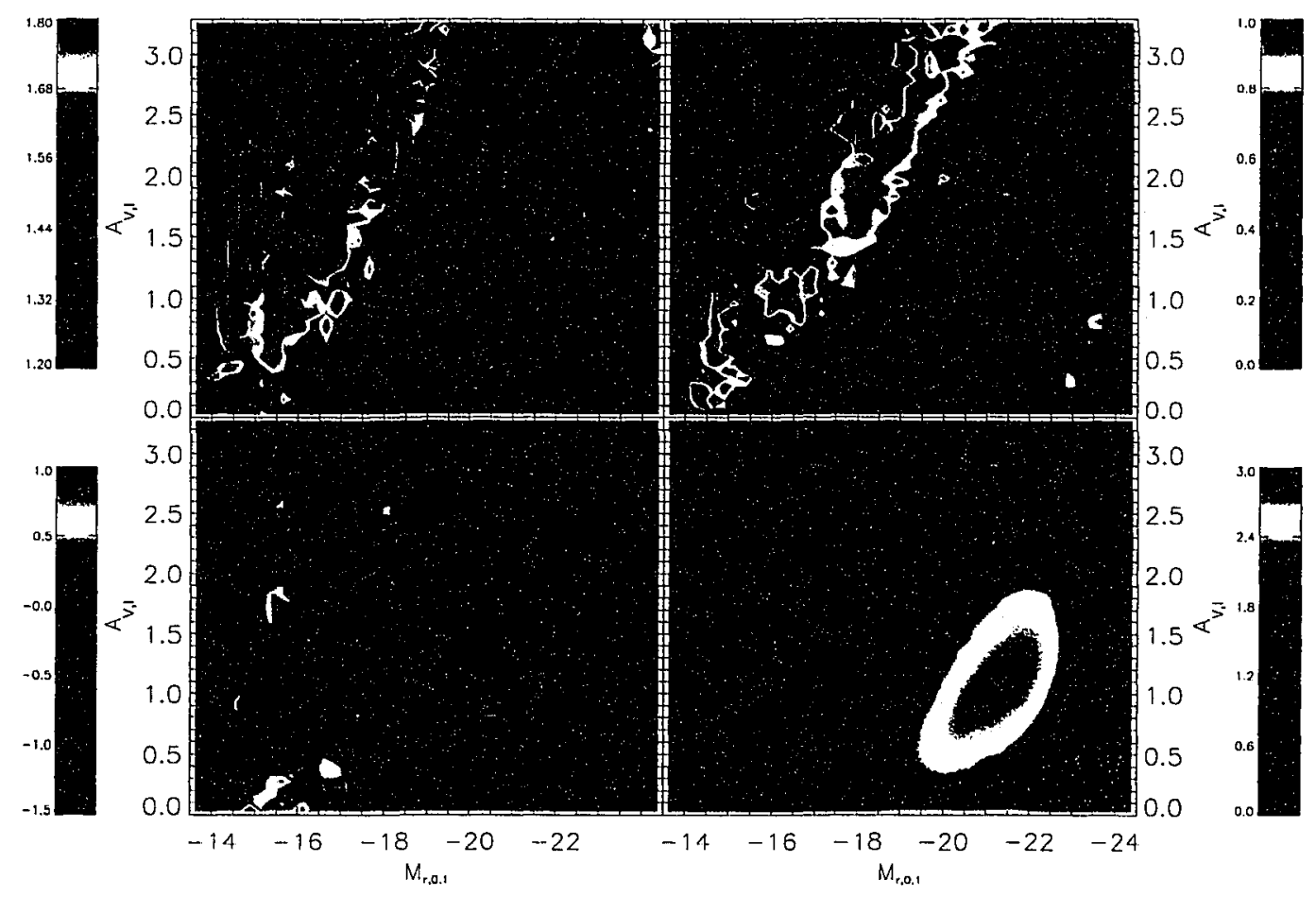

Figure 3.8 Values of fitted parameters for 2-D bins cut on luminosity and emission line extinction. The bins extend 0.25 magnitudes in luminosity and 0.06 magnitudes in extinction. The description is identical to Figures 3.6 and 3.7 , as are the shading levels. 


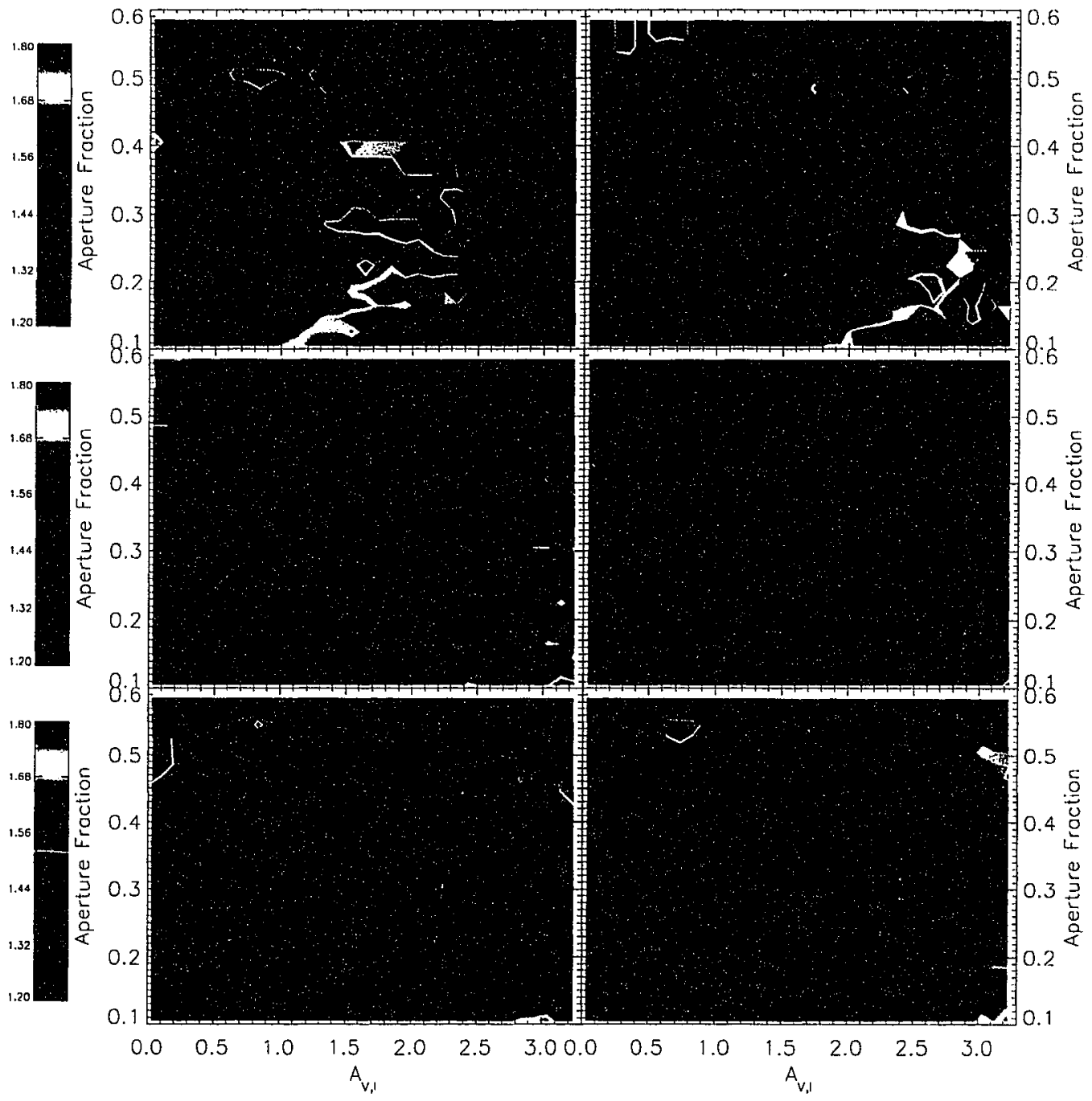

Figure 3.9 Best fitting $\Gamma$ values as a function of aperture fraction and measured emission line extinction for six volume limited luminosity bins. The 2-D bins extend 0.1 magnitudes in extinction and $2 \%$ in aperture fraction. The volume limited bins are defined as described in Figure 3.5. The white contour shows the area where the 2-D bins contain at least 50 galaxies. The shading levels are described by the color bars on the left. 


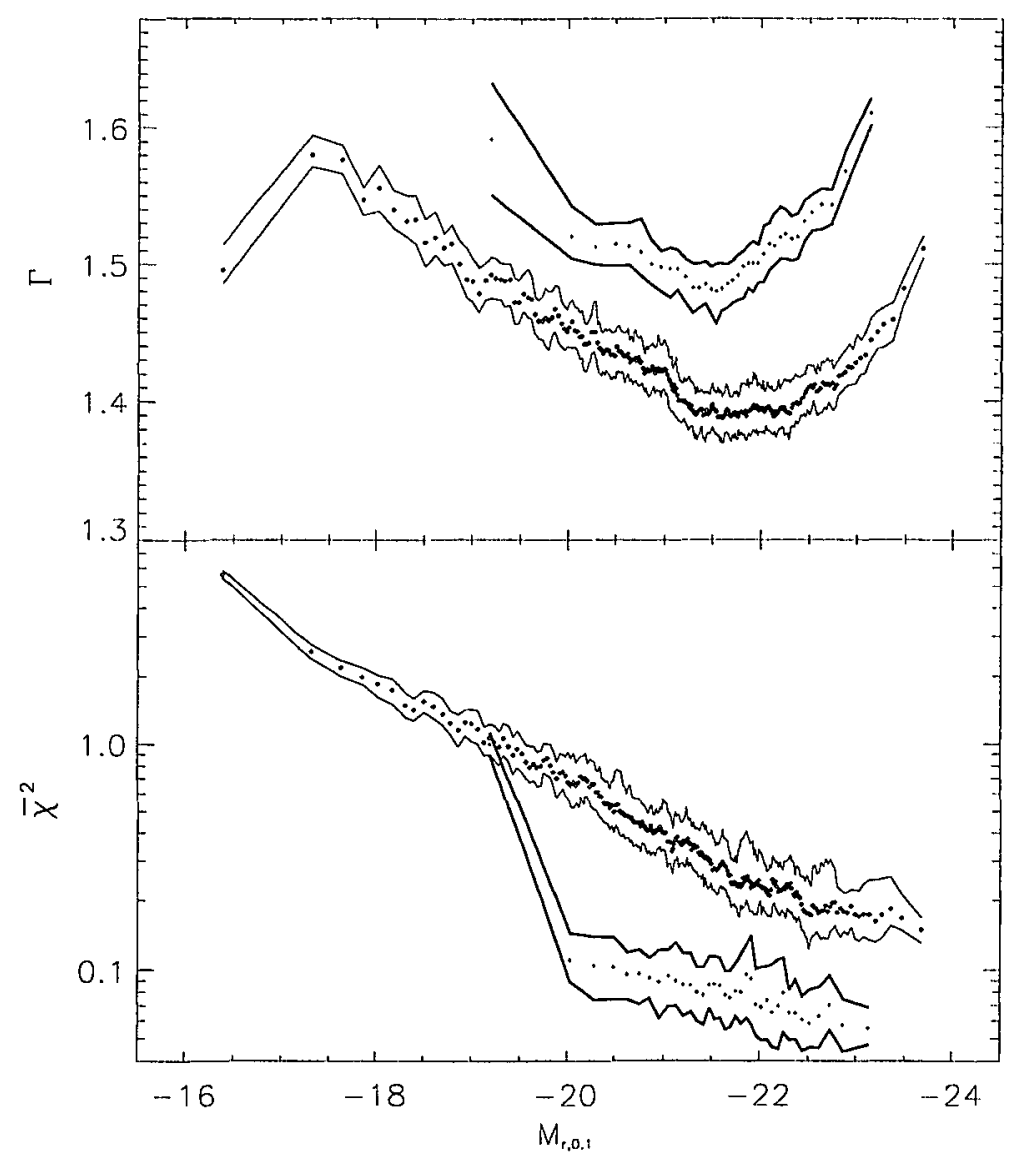

Figure 3.10 MC simulation results binned by $\mathrm{M}_{r, 0.1}$ for the 111,806 galaxies in the star forming class (diamonds) and the 18,796 galaxies in the low $\mathrm{S} / \mathrm{N}$ star forming class ( + ) of Brinchmann, et al. (2004). Each symbol represents a bin of 500 galaxies. The thin lines represent the upper and lower $95 \%$ confidence region measured for each star forming bin, while the thick lines do so for the low $\mathrm{S} / \mathrm{N}$ bins. Top panel: The best fitting $\Gamma$ values as a function of $r$-band luminosity. Lower panel: The $\overline{\chi^{2}}$ values for each luminosity plotted on a log scale. Because the sample is dominated by the star forming class the results for these galaxies is very similar to the result for the whole sample in Figure 3.2. 


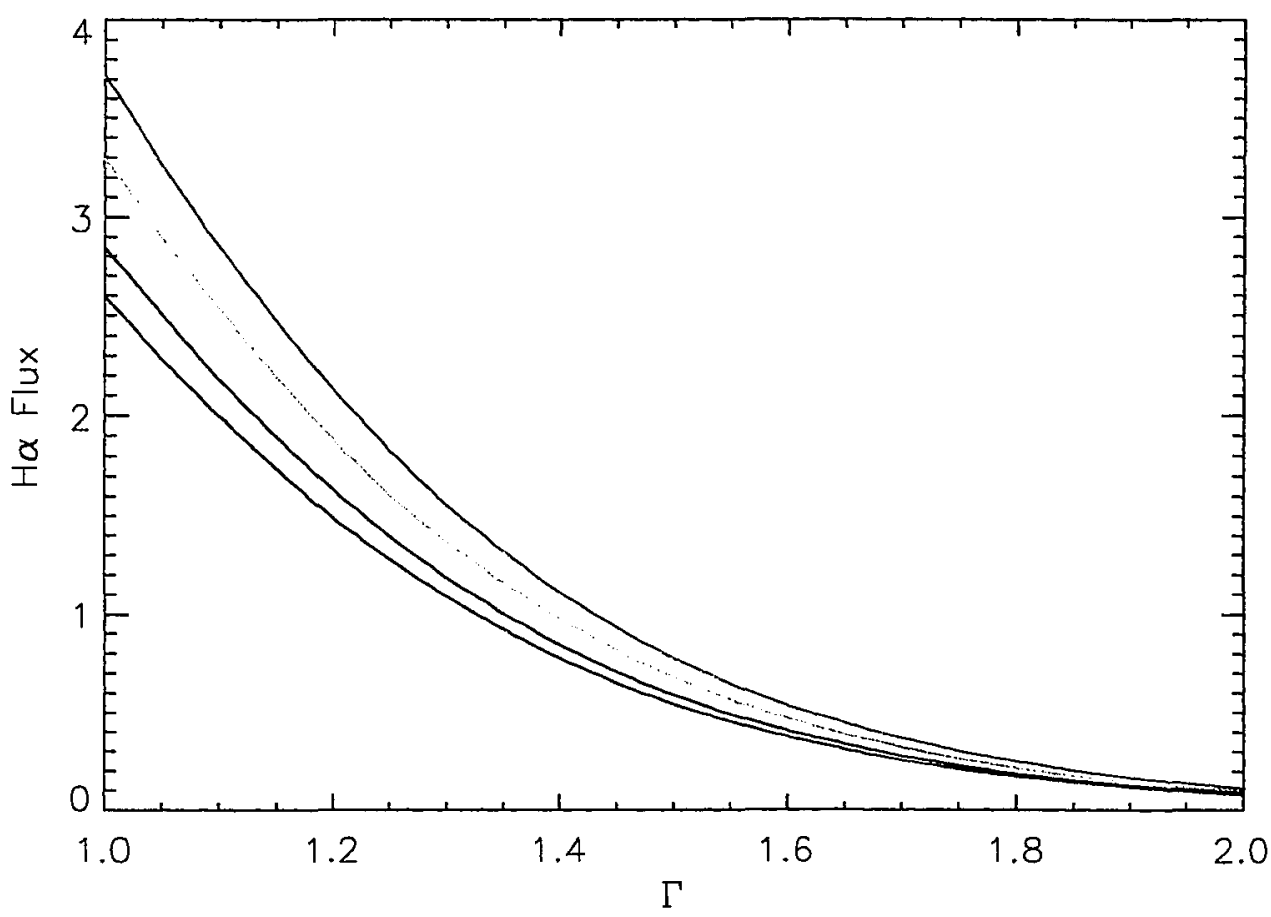

Figure $3.11 \mathrm{H} \alpha$ flux as a function of $\Gamma$ and metallicity at constant SFR. As the SFR is often measured from the $\mathrm{H} \alpha$ flux this plot demonstrates the degree to which the measured SFR can deviate from the true SFH in the presence of IMF variations. The $\mathrm{H} \alpha$ flux is plotted as a function of $\Gamma$ for four metallicity models: $Z=0.005$ (red), $Z=0.010$ (violet), $Z=0.020$ (blue) and $Z=0.025$ (green). The $\mathrm{H} \alpha$ flux is plotted in arbitrary units normalized to $\Gamma=1.35$ and $Z=0.020$. 


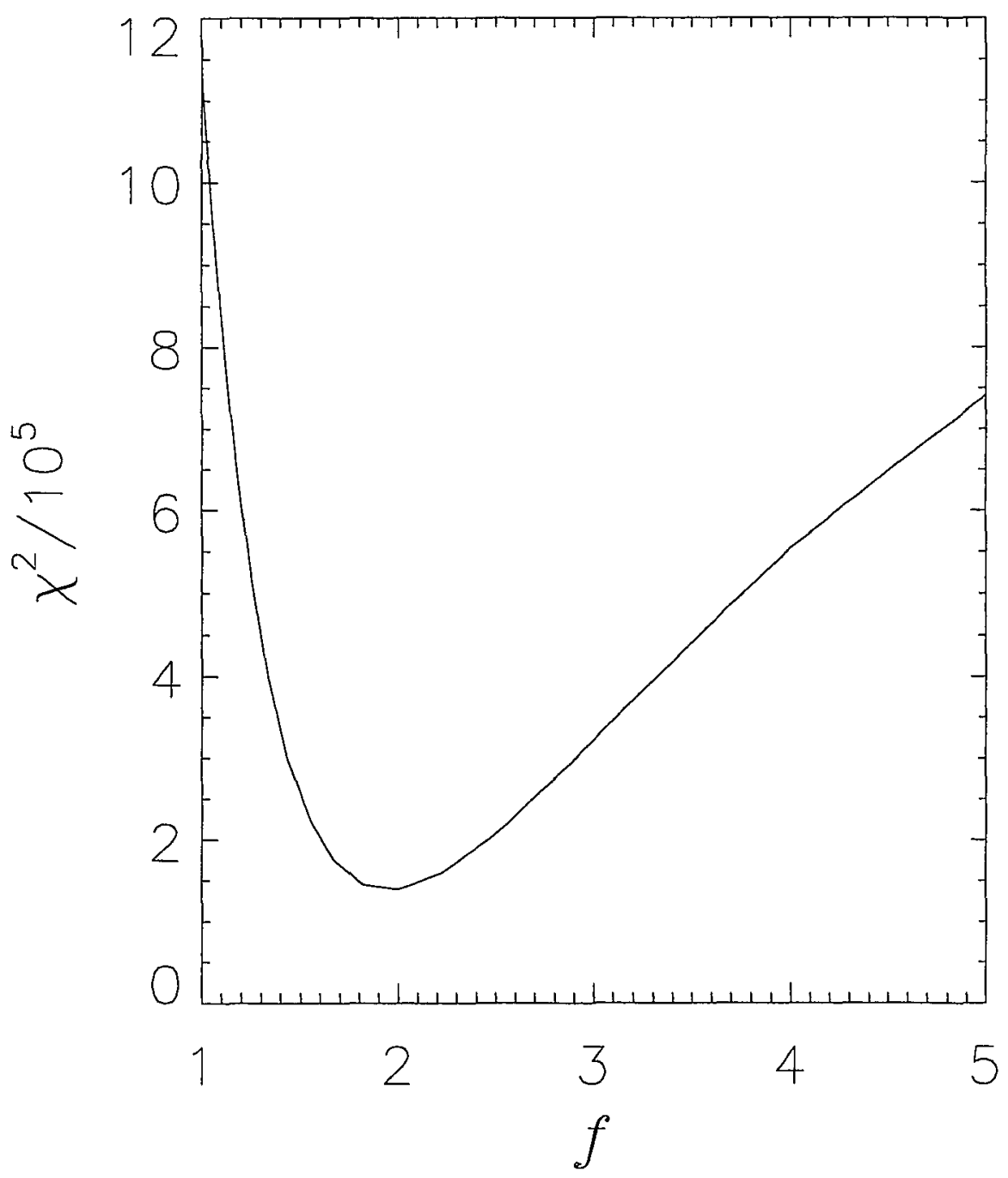

Figure 3.12 Similar to Figure 3.1, this figure shows the value of $\chi^{2}$ as a function of the assumed $f$ ratio for the entire sample assuming $\Gamma=1.35$. 


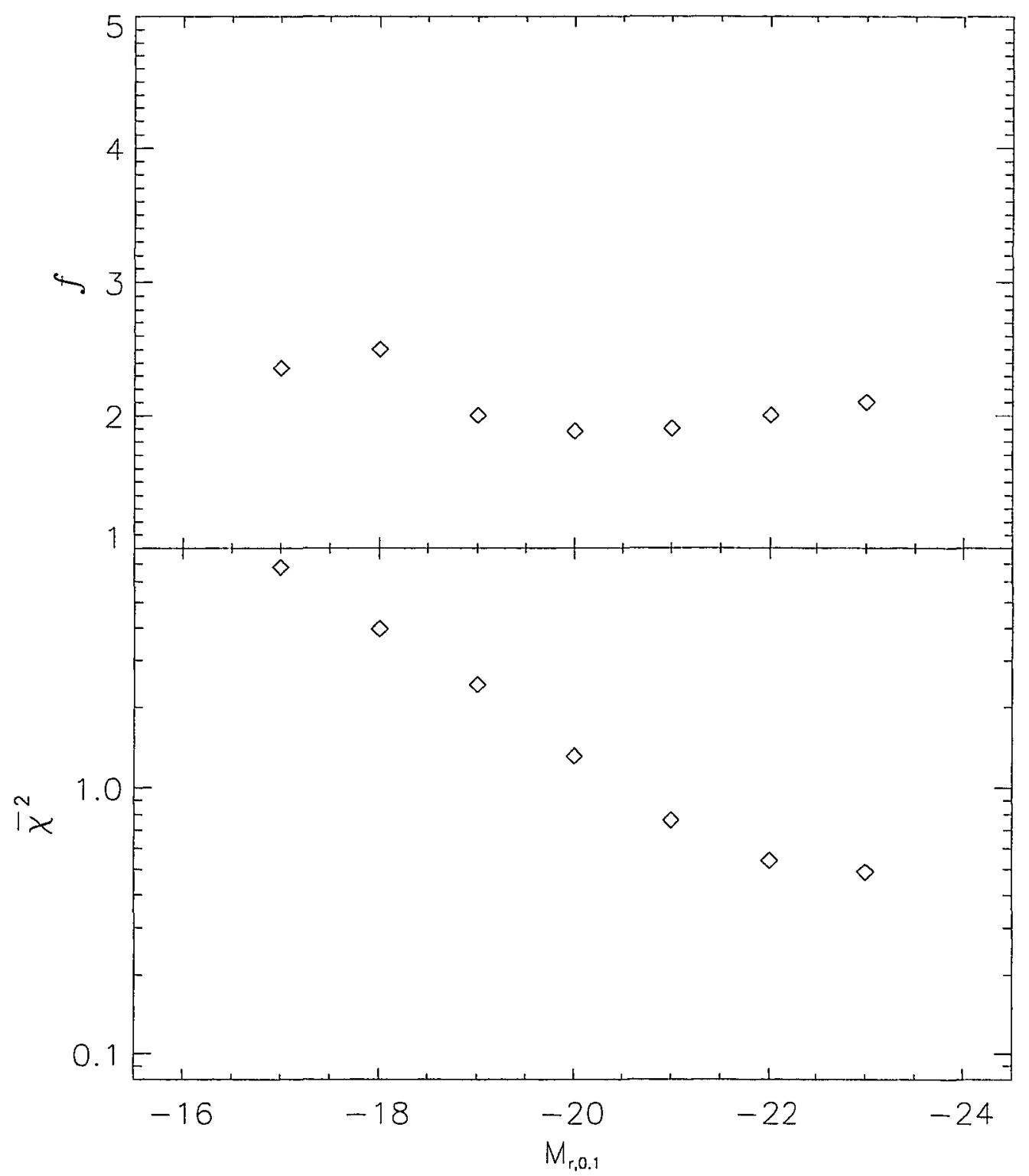

Figure 3.13 Upper panel: The best fitting $f$ ratio, assuming a universal Salpeter IMF, as a function of luminosity. The luminosity bins are volume limited and the same as in Figure 3.5. Lower panel: The corresponding modified $\overline{\chi^{2}}$ values as a function of luminosity. 


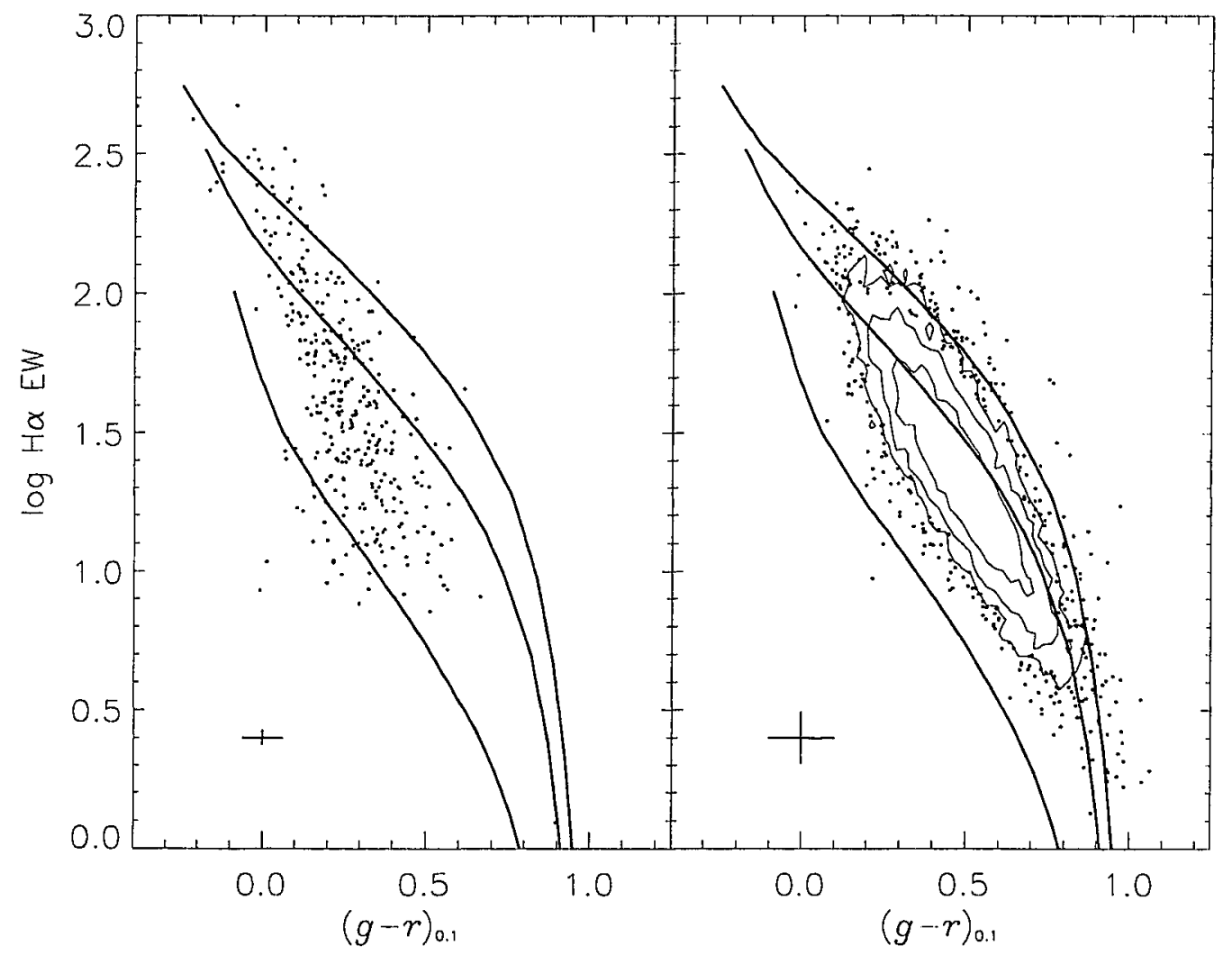

Figure 3.14 Distribution in $(g-r)_{0.1}-\log (\mathrm{H} \alpha \mathrm{EW})$ space of the 329 galaxies in the volume limited $\mathrm{M}_{r, 0.1}=-17$ bin (left) and the 8,049 galaxies in the $\mathrm{M}_{r, 0.1}=-23$ bin (right). The contour levels and other descriptions are identical to Figure 2.1. 


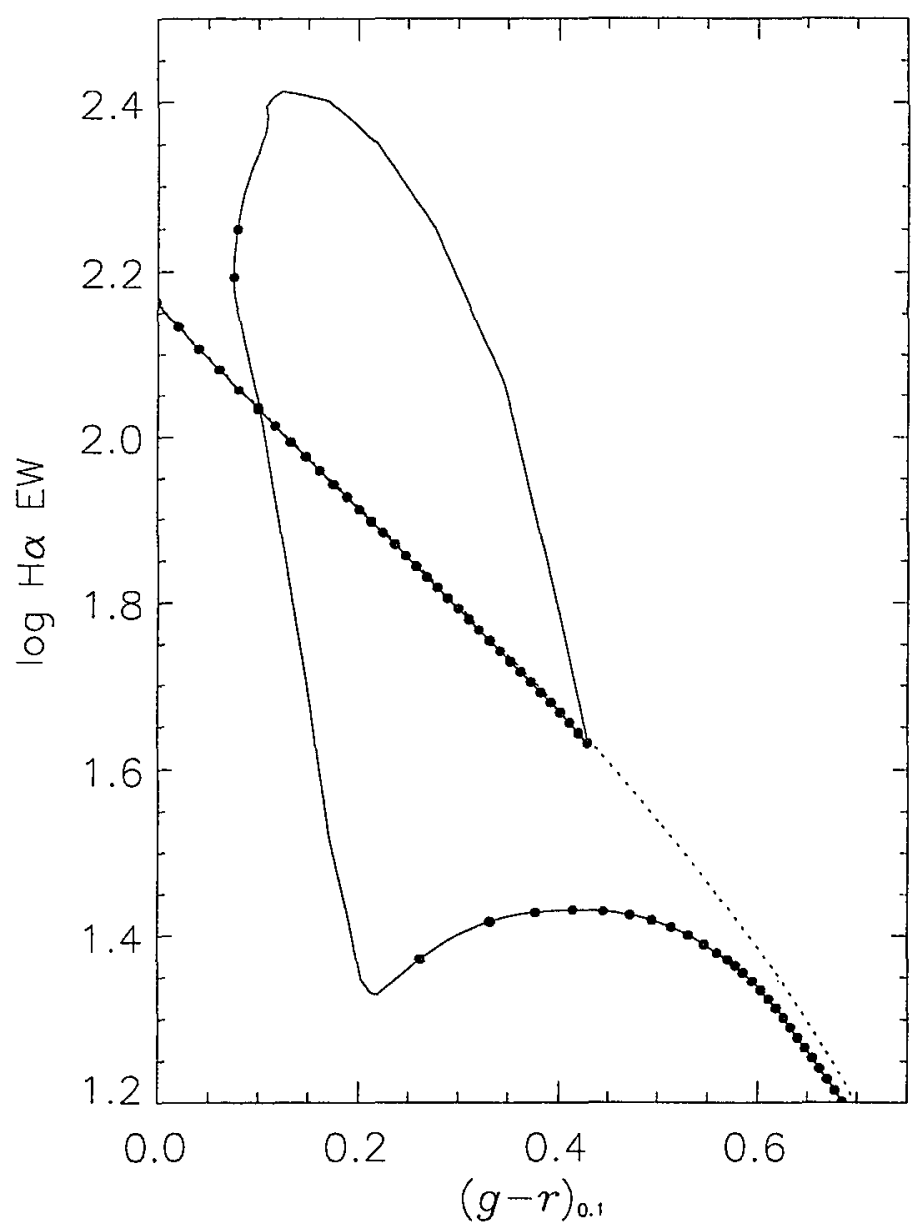

Figure 3.15 Color and $\mathrm{H} \alpha \mathrm{EW}$ values as a function of age for a model galaxy experiencing: a burst (solid line). The model galaxy has solar metallicity, $\Gamma=1.35$, and an exponentially decreasing SFH with $\tau=2.15 \mathrm{Gyr}$. The burst occurs at an age of $4.113 \mathrm{Gyr}$, lasts $250 \mathrm{Myr}$ and has a strength of $10 \%$ of the total stellar mass. The age of the galaxy increases from the upper left to lower right before the bursts and the black dots appear on the track at $100 \mathrm{Myr}$ intervals. The dotted line shows the track for the model had a burst not occurred. 


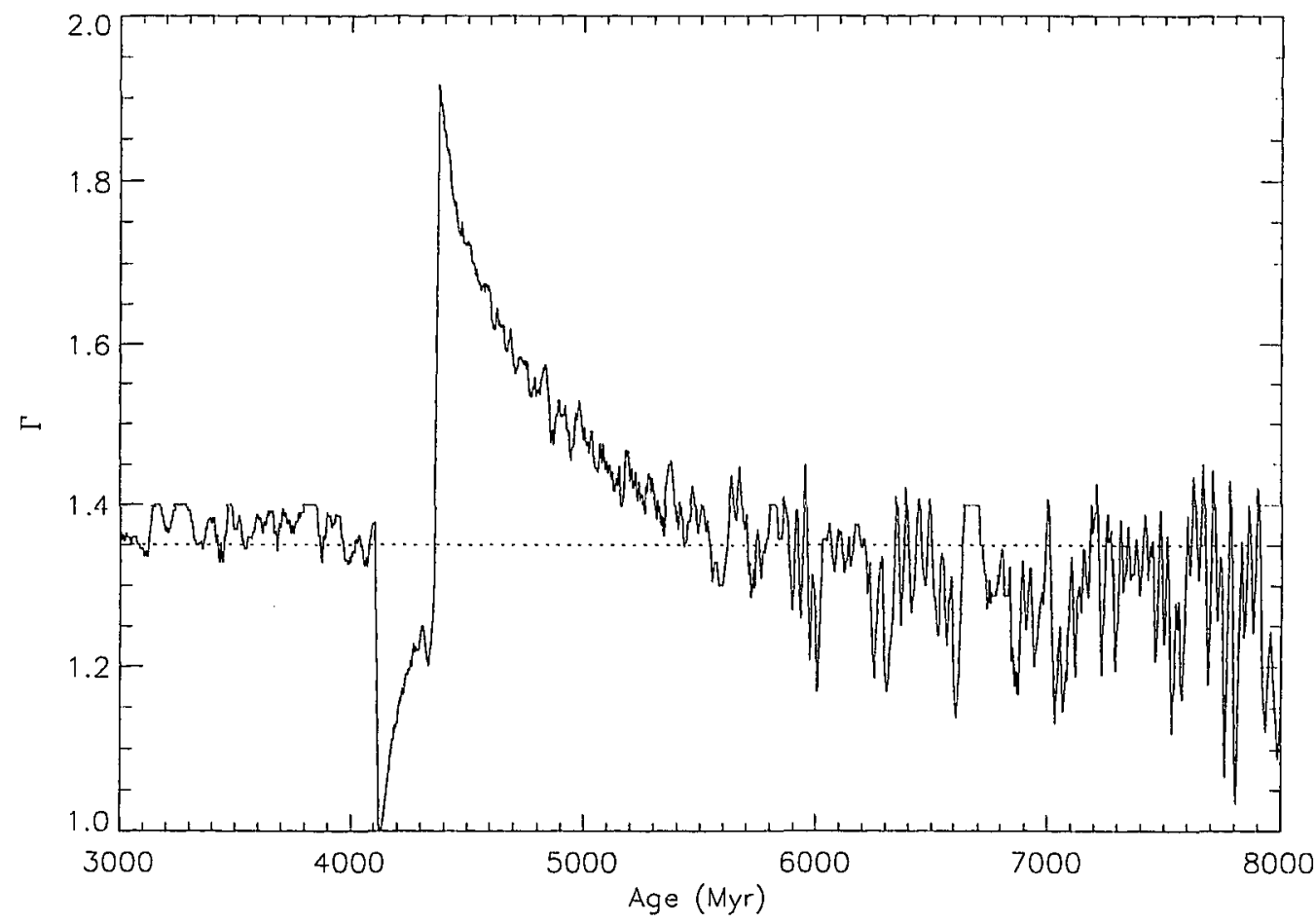

Figure 3.16 Best fitting IMF slope as a function of age for the model galaxy in Figure 3.15, which experiences a burst of star formation at an age of 4.113 Gyr. The dotted line indicates the underlying IMF model $(\Gamma=1.35)$. The $\Gamma$ values have been boxcar smoothed by $20 \mathrm{Myr}$ for clarity. 


\section{Single Burst Models}

The simplest burst model is that of a single burst at a random time on top of our smooth exponential SFHs. A grid of $1000 \mathrm{SFHs}$ was constructed by first selecting one of the 24 smoothly varying SFHs at random. A burst lasting $200 \mathrm{Myr}$ was superposed on the SFH at a time selected uniformly at random over a range of 12.5 Gyr. The strength of the burst was randomly selected up to $40 \%$ of the total stellar mass, with preference given to smaller bursts. The colors and EWs of these SFHs were calculated at $1 \mathrm{Myr}$ intervals over $12.5 \mathrm{Gyr}$ for the IMFs $\Gamma=1.35$ and 1.80 and a fixed metallicity of $Z=0.01$. This is based on the luminosity-metallicity relationship in Tremonti, et al. (2004) for $M_{r, 0.1}=-17$ galaxies. Plotting these models in the color- $\mathrm{H} \alpha \mathrm{EW}$ plane shows that all observed data points are covered by either IMF.

To test whether the observed distribution of points in the $\mathrm{M}_{r, 0.1}=-17$ bin can be explained by bursting SFHs the MC techniques of $\S 2.4$ were used. The models of $\S 2.3$ were replaced with the grid of single burst models. The $100 \mathrm{MC}$ simulations were constructed as described earlier, but using only the $329 \mathrm{M}_{r, 0.1}=-17$ galaxies as a basis. The analysis yielded $\overline{\chi^{2}}=0.027$ for $\Gamma=1.35$ and $\overline{\chi^{2}}=0.020$ for $\Gamma=1.80$, both of which are over fits.

This shows than an individual galaxy can be fit with an arbitrary IMF given the freedom to choose a SFH. However, our advantage is that we have many galaxies and the distribution of the properties of the best fit models can be shown to be implausible.

Figure 3.17 demonstrates the problem with the single burst model. On the left of the figure the distribution of the best fitting burst strengths are plotted as a fraction of the total stellar mass formed. At right is the distribution of the best fitting times from the 
burst onset. For example, a galaxy which best fits a model with a burst at $1.000 \mathrm{Gyr}$ at au age of $1.211 \mathrm{Gyr}$ has a time from burst onset of $211 \mathrm{Myr}$. This measure is used because in an investigation of the effects of bursts the age relative to the burst is more important than the age given that the bursts occur at different, random times across the models.

For both $\Gamma=1.35$ and $\Gamma=1.80$ the number of objects best fit by a model prior to the burst is $11+3 /-5$, or $3 \%$, and are not plotted. In both cases the distributions of the best fitting ages and ages at which the burst begins are roughly uniform. Given this fact it is expected that half of the galaxies should be best fit by a pre-burst model. Furthermore the right hand panel shows sharp, significant discontinuities in the distribution of best fitting time from burst onset. Again, viewed at random times this distribution should be uniform but is highly peaked in the $25 \mathrm{Myr}$ at the start of the burst and the $25 \mathrm{Myr}$ just after the burst ends. In both cases the errors bars show that the discontinuities are significant. In the case of $\Gamma=1.35,3.5$ times as many galaxies are in the $200 \mathrm{Myr}$ after the burst ends than the $200 \mathrm{Myr}$ during it and this $400 \mathrm{Myr}$ accounts for $57 \%$ of all galaxies. Although our sample is $r$-band selected the stars in the 0.7 to $3 \mathrm{M}_{\odot}$ range which dominate the red continuum in the red giant phase do not start to leave the main sequence for $300 \mathrm{Myr}$. The sharp increase in galaxies fit at $200 \mathrm{Myr}$ after the burst cannot be due to a selection effect. Assuming a universal IMF this points to a strong coordination of SFHs across a population of galaxies unrelated in space. These arguments show that while a single burst model can fit the data extremely well, it does not do so in a physically self-consistent fashion. 


\section{Multiple Burst Models}

To find a physical motivation for SFH models for low luminosity galaxies we look towards the Local Group. There have been a number of recent studies of the SFHs of local dwarf galaxies which use HST to get color-magnitude diagrams (CMD) of resolved stellar populations. The SFH is determined by fitting isochrones to the CMD. The sample here is biased by Local Group membership and by what galaxies have been observed to date. The galaxies mentioned here give a point of reference rather than a well-defined distribution of SFHs.

The blue compact dwarf (BCD) UGCA 290 was found to quiescently form stars over the past Gyr up until a ten-fold increase in SFR from 15 to $10 \mathrm{Myr}$ ago which more recently has decreased to a quarter of its peak value (Crone, et al. 2002). The dwarf irregular IC 1613, which is relatively isolated and non-interacting, was found to have SFR enhanced by a factor of 3 from 3 to 6 Gyr ago without evidence of strong bursts (Skillman, et al. 2003). The dwarf irregular NGC 6822, also relatively isolated, is found to have a roughly constant SFH (Wyder 2003). The BCD NGC 1705 is found to be gasping- a SFH marked by moderate activity punctuated by short periods of decreased star formation (Annibali, et al. 2003). The authors also note that NGC 1705 is best fit by an IMF with $\Gamma=1.6$. NGC 1569 likely experienced three strong bursts in the last Gyr as well as a quiescent phase from 150 to $300 \mathrm{Myr}$ ago (Angeretti, et al. 2005).

Inspired by the preceding Local Group SFHs we constructed six SFH classes with multiple bursts. These SFH classes are described in Table 3.2. Each class starts with an underlying smooth SFH. SFHs \#1 \& 2 have no star formation, \#3 \& 4 have a constant 
Table 3.2. Multi-burst SFH Models

\begin{tabular}{lccccl}
\hline Name & Type & $\begin{array}{c}\text { Length } \\
\text { (Myr) }\end{array}$ & $\begin{array}{c}\text { Relative } \\
\text { Strength }\end{array}$ & $\begin{array}{c}\text { Spacing } \\
(\mathrm{Gyr})\end{array}$ & $\begin{array}{c}\text { Underlying } \\
\text { SFH }\end{array}$ \\
\hline & & & & & \\
SFH 1 & burst & 200 & - & 3 & none \\
SFH 2 & burst & 200 & - & 1 & none \\
SFH 3 & burst & 200 & 4.0 & 3 & constant \\
SFH 4 & gasp & 200 & 0.0 & 1.5 & constant \\
SFH 5 & gasp & 200 & 0.1 & 1.5 & exponential \\
SFH 6 & burst & 200 & 5.0 & 1.5 & exponential \\
\hline
\end{tabular}

SFH and \#5 \& 6 have exponentially decreasing SFHs like those previously described. Star formation discontinuities are then superimposed on top of the smooth SFHs. These discontinuities are in the form of increased (bursts) or decreased (gasps) star formation for periods of $200 \mathrm{Myr}$. The time and spacing of the discontinuities is random with the mean interval between bursts listed in Table 3.2 .

For each SFH class described above we randomly generated 1,000 SFHs. Colors and EW widths were calculated for each SFH using $\Gamma=1.35$ and $Z=0.01$. According to the SDSS mass-metallicity relationship (Tremonti, et al. 2004) galaxies at $M_{r, 0.1}=-17$ will on average have $Z=0.01$.

We then repeated our $\chi^{2}$ analysis with the $100 \mathrm{MC}$ simulations of the $\mathrm{M}_{r, 0.1}=-17$ galaxies in the same manner as for the single burst models. The results of our analysis for each of the six SFH classes are shown in Figure 3.18. SFH 5, the gasps on top of exponential SFHs, is the best fit with $\overline{\chi^{2}}=0.09$. Extended periods of no star formation punctuated by 
bursts (SFHs \# 1 \& 2) do not fit the data.

As was the case for the single burst models an unreasonable fraction of galaxies are best fit by SFHs in the $20 \mathrm{Myr}$ immediately following a burst or the first $20 \mathrm{Myr}$ of a gasp. If the SFH models are reasonable we should see roughly equal numbers of galaxies in each time bin. There is no reason why all of the low luminosity galaxies across the large volume of space in the SDSS footprint should have experienced coordinated bursts. However each panel of Figure 3.18 has at least $40 \%$ of the galaxies in one $20 \mathrm{Myr}$ bin.

One explanation for this is that it is an artifact of our sample being selected in the $r$ band. However spectral synthesis models show that for instantaneous bursts of star formation the $r_{0.1}$ magnitude is brightest at the burst time and decays smoothly for a range of $\Gamma$. If anything it is more likely to catch galaxies during a burst rather than after or after a gasp instead of during one.

Regardless of the SFH model the presence of blue galaxies with low $\mathrm{H} \alpha \mathrm{EW}$ requires a recent discontinuity in the SFR for $\Gamma=1.35$. Based on the evolution of the $r_{0.1}$ band luminosity we expect to see a similar number of galaxies with excess $\mathrm{H} \alpha \mathrm{EWs}$. The fact that these galaxies are missing shows that the discontinuous SFH models do not match our observations. Therefore IMF variations are a more likely explanation for the observed distribution of $\mathrm{M}_{r, 0.1}=-17$ galaxies.

\section{Recovering $\Gamma$ from Synthetic Data}

As a last exercise the best fitting SFH models from the previous section can be run forward to see if the correct IMF can be recovered. For each SFH model grids 10,000 data points were chosen by selecting a random SFH and a uniformly distributed age. Normal 
errors were added using the error characteristics of the $M_{r, 0.1}=-17$ bin. This synthetic: data was analyzed in the same fashion as the real data in the earlier sections. For the single burst models the recovered IMF models for $\Gamma=1.35$ and 1.80 were 1.34 and 1.79 respectively with best fitting $\overline{\chi^{2}}=0.80$ and 0.65 . For SFH 5 the recovered IMF was also 1.34 with $\overline{\chi^{2}}=1.5$. In all three cases the correct IMFs were recovered although the fit was worsened by by the burst activity. This reinforces the difficulty in producing enough blue, low $\mathrm{H} \alpha \mathrm{EW}$ galaxies to match the observed data with simple SFH models.

\section{$3.2 \quad \mathrm{H} \delta_{\mathrm{A}}$ Absorption}

In the previous sections we have expanded on the K83 method and exploited the $\mathrm{H} \alpha$ and color information as much as possible. In the bias section we found that various possible biases do not fully explain either the increased values of $\Gamma$ or the poor fit to a single IMF in the lowest luminosity bins. In the SFH section we found that an arbitrary $\Gamma$ value coupled with a plausible SFH with bursts or gasps can account for the position of any individual galaxy in the color- $\mathrm{H} \alpha \mathrm{EW}$ plane. However taking the population of $\mathrm{M}_{r, 0.1}=-17$ galaxies together necessitates an incredibly unlikely coordination of SFHs across the disparate group of objects. This points to the extraordinary conclusion that while the IMF may be universal across luminous galaxies, it is not in fact universal in low luminosity galaxies. Such an extraordinary claim would ideally be backed by extraordinary

evidence. In this section we take a look beyond the K83 method for some reinforcement of our result.

The $\mathrm{H} \delta$ absorption feature can be used to gain additional insight into the nature 
of stellar populations. $\mathrm{H} \delta$ absorption is due to absorption lines form stellar photospheres. The Balmer absorption lines are most prominent in A stars and weaken due to the Saha equation for both hotter and cooler stars. As such the $\mathrm{H} \delta$ absorption is a proxy for the fraction of light of a stellar population being supplied by A stars, and to a lesser extent $\mathrm{B}$ and $\mathrm{F}$ type stars. In a stellar population of a uniform age the $\mathrm{H} \delta$ absorption will peak after the $\mathrm{O}$ and $\mathrm{B}$ stars burn out, but before the $\mathrm{A}$ stars leave the main sequence. For this reason the strength of $\mathrm{H} \delta$ can be used help determine the age of a population or to detect bursts of star formation which occurred around $1 \mathrm{Gyr}$ in the past.

Worthey \& Ottaviani (1997) describes two different methods for measuring $\mathbf{H} \delta$ absorption. The $\mathrm{H} \delta_{\mathrm{F}}$ definition is tuned to most accurately measure the $\mathrm{H} \delta$ absorption from $\mathrm{F}$ stars. The $\mathrm{H} \delta_{\mathrm{A}}$ definition has a wider central bandpass to match the line profiles of A stars. They state that the $\mathrm{H} \delta_{\mathrm{A}}$ definition is better to use for galaxies because it is less noisy in low $\mathrm{S} / \mathrm{N}$ galaxies and velocity dispersion acts to widen absorption features. On the downside the narrower $\mathrm{H} \delta_{\mathrm{F}}$ definition is much more sensitive to population age where it can be used. Worthey \& Ottaviani (1997) observationally determines the range of $\mathrm{H} \delta_{\mathrm{A}}$ values to be from 13 for $\mathrm{A} 4$ dwarf stars to -9 for M-type giant stars.

$\mathrm{H} \delta_{\mathrm{A}}$ values can be measured from the SDSS spectra. Like the $\mathrm{H} \alpha \mathrm{EW}$ values our $\mathrm{H} \delta_{\mathrm{A}}$ values come from Tremonti, et al. (2004) instead of the SDSS pipeline.

Figure 3.19 compares the distribution of the $\mathrm{H} \delta_{\mathrm{A}}$ values for the $\mathrm{M}_{r, 0.1}=-17$ and $M_{r, 0.1}=-23$ luminosity bins. For reference recall that Figure 3.14 plots the color and $H \alpha$ EWs for these two bins. The difference between the high and low luminosity bins is clear. Gaussian profiles can be fit to both distributions. For the $\mathrm{M}_{r, 0.1}=-23$ bin 
the standard deviation of the profile is twice as large as the measurement error in $\mathrm{H} \delta_{\mathrm{A}}$ suggesting that the true distribution lias a range of values. Assuming both the errors and underlying distribution are Gaussian the distribution of $\mathrm{H} \delta_{\mathrm{A}}$ for the luminous galaxies is centered at $\mathrm{H} \delta_{\mathrm{A}}=3.4$ with a standard deviation of 1.5 . By contrast, the Gaussian fit to the $\mathrm{M}_{r, 0.1}=-17$ bin has the same standard deviation as the median error in the galaxies. This is consistent with nearly all of the galaxies having $\mathrm{H} \delta_{\mathrm{A}}=6.1$.

This shows that the fainter galaxies on average have significantly larger fractions of A-type stars amongst their stellar populations. It also shows a seemingly unlikely coordination of $\mathrm{H} \delta_{\mathrm{A}}$ in the low luminosity galaxies, reminiscent of the earlier suggestion of coordinated SFHs. The question then becomes why?

For a possible explanation we look again to the models. Modeling the behavior of $\mathrm{H} \delta_{\mathrm{A}}$ requires an extra step. The standard PEGASE.2 models do not have the required resolution to accurately measure the $\mathrm{H} \delta_{\mathrm{A}}$ index. This is remedied with the use of the PEGASE-HR code (Le Borgne, et al. 2004) which uses a library of echelle spectra of 1503 stars to calculate spectral synthesis models with $R=10,000$ over the range of 4000 to $6800 \AA$. Using PEGASE-HR in the low resolution mode yields the same results as PEGASE.2, and the same input parameters are used for both codes. The models here are the same as those described earlier in the models section, but have been recalculated using PEGASE-HR to allow $\mathrm{H} \delta_{\mathrm{A}}$ measurements.

Figure 3.20 shows the behavior of the $\mathrm{H} \delta_{\mathrm{A}}$ index as a function of age for four different IMF models, $\Gamma=1.00,1.40,1.70$ and 2.00. For each IMF models of all metallicities and smooth SFHs are plotted for each age. The qualitative behavior of all models is the 
same. The $\mathrm{H} \delta_{\mathrm{A}}$ holds steady for the first 20 to $40 \mathrm{Myr}$ before increasing to a peak value at $700 \mathrm{Myr}$ to $1 \mathrm{Gyr}$ and then falls off. Prior to reaching the peak value for each IMF the metallicity has the strongest effect on the $\mathrm{H} \delta_{\mathrm{A}}$ index. At this point the lowest metallicity galaxies have the highest $\mathrm{H} \delta_{\mathrm{A}}$. After the peak the SFH has the strongest effect with the constant and increasing SFHs maintaining higher $\mathrm{H} \delta_{\mathrm{A}}$ values.

After a few Gyr the differences in $\mathrm{H} \delta_{\mathrm{A}}$ between models with different IMFs disappear. Prior to that there are three main differences. First, the peak $H \delta_{\mathrm{A}}$ values are higher for larger values of $\Gamma$. For $\Gamma=2.00 \mathrm{H} \delta_{\mathrm{A}}$ reaches a maximum value of nearly 8 and the maximum value is similar for all metallicities. For $\Gamma=1.00$ the maximum value ranges from 4.5 to 6 depending on the metallicity. This is due to the fact that the steeper IMFs have fewer luminous massive stars to dilute the $\mathrm{H} \delta$ absorption features from the A star population. Secondly, the low $\Gamma$ models have $\mathrm{H} \delta_{\mathrm{A}}$ values that start their initial increases at a later time. Lastly the low $\Gamma$ models reach their peak values later in time than those with fewer massive stars.

To compare the $\mathrm{H} \delta_{\mathrm{A}}$ values for the $\mathrm{M}_{r, 0.1}=-23$ and -17 bins to the models the range of the middle $90 \%$ values from Figure 3.19 for each bin are overlaid on Figure 3.20. Once again the $\mathrm{M}_{r, 0.1}=-23$ bin is in good agreement with our assumption of a universal IMF and smooth SFH. The range of $\mathrm{H} \delta_{\Lambda}$ values can be accomplished with a single Salpeter-like $\Gamma=1.40$ IMF with only the proviso that most galaxies be older than a few Gyr or younger than $300 \mathrm{Myr}$. However the exact same statement can be made for $1.0<\Gamma<2.0$ so $\mathrm{H} \delta_{\mathrm{A}}$ provides only a constraint on the age of the most luminous galaxies, but not the IMF. 
For the $\mathrm{M}_{r, 0.1}=-17$ galaxies the distribution of $\mathrm{H} \delta_{\mathrm{A}}$ camnot be achieved with the shallowest IMFs investigated under the assumption of smooth SFHs. However Salpeter and stecper IMFs can be accomplished. What changes is the range of ages over which the models have the correct $\mathrm{H} \delta_{\mathrm{A}}$. Steeper IMFs require that the galaxies be either older than a few Gyr or $200 \mathrm{Myr}$ old to accommodate the observed $H \delta_{\mathrm{A}}$ values. Most troubling is that there does not appear to be any reason why $\mathrm{H} \delta_{\mathrm{A}}$ should stack up at 6.1 for the $\mathrm{M}_{r, 0.1}=-17$ galaxies.

Discontinuous SFHs may also play a roll in the distribution of $\mathrm{H} \delta_{\mathrm{A}}$. This possibility is investigated in figure 3.21 which shows the relationship between multiple bursts of star formation and $\mathrm{H} \delta_{\mathrm{A}}$ for two of the least offensive multiple burst models classes. The underlying exponential SFHs are the same in each panel but the top panel has gasps, as an example of $\mathrm{SFH} \# 5$, and the bottom has bursts of star formation (SFH \#6).

During a star formation gasp $\mathrm{H} \delta_{\mathrm{A}}$ rapidly increases because there are much fewer $\mathrm{O}$ stars with luminous, featureless continuua to wash out the Balmer absorption lines. At the end of the gasp $\mathrm{H} \delta_{\mathrm{A}}$ quickly returns to nominal levels. During a burst of star formation $\mathrm{H} \delta_{\mathrm{A}}$ immediately drops due to an excess of $\mathrm{O}$ stars. Shortly after a burst $\mathrm{H} \delta_{\mathrm{A}}$ is significantly higher than preburst levels. This is because there is an excess of A stars with strong Balmer absorption left over from the burst but the corresponding $O$ stars have all burned out. The inflated $H \delta_{\mathrm{A}}$ values decay to nominal levels over a Gyr. At late ages there are large underlying populations of old stars which damp the effects of SFH discontinuities on $\mathrm{H} \delta_{\Lambda}$

The vertical dotted lines in figure 3.21 show the $20 \mathrm{Myr}$ intervals around SFH 
discontinuities which are so heavily favored by the previous analysis in figure 3.18 . In the multiburst models the $\mathrm{H} \delta_{\mathrm{A}}$ reach peak values over a time range of $200 \mathrm{Myr}$, much longer than the $20 \mathrm{Myr}$ intervals favored earlier. Furthermore $\mathrm{H} \delta_{\mathrm{A}}$ does not reach its peak values until after the $20 \mathrm{Myr}$ intervals. The behavior of $\mathrm{H} \delta_{\mathrm{A}}$ in the presence of SFH discontinuities provides no need for galaxies to stack up in the narrow $20 \mathrm{Myr}$ intervals seen in the earlier multiple burst section, nor do these models suggest why the low luminosity galaxies are consistent with a single value of $\mathrm{H} \delta_{\mathrm{A}}$.

There is no satisfactory model to account for the $\mathrm{H} \delta_{\mathrm{A}}$ distribution of the low luminosity galaxies. However the SFH results from the previous section strongly suggest that the incredible coordination of discontinuities is highly unlikely. The low luminosity galaxies are most likely the result of a mix of IMFs.

\subsubsection{Three Parameter Monte Carlo Analysis}

As seen in the previous section analyzing the $\mathrm{H} \delta_{A}$ values alone provides some additional clues about the relationship between galaxy luminosity and the IMF. By analyzing $\mathrm{H} \delta_{A}$ with the color and $\mathrm{H} \alpha \mathrm{EW}$ simultaneously a deeper understanding can be reached that is not immediately obvious from looking at the parameters individually.

In this section the earlier MC analysis is repeated with the only change being the inclusion of the $\mathrm{H} \delta_{A}$ values. The $\chi^{2}$ value of equation 2.9 is modified to:

$$
\chi_{i}^{2}(\Gamma, Z, t, \psi)=\left(\frac{c_{i}-c(\Gamma, Z, l, \psi)}{\sigma_{c_{i}}}\right)^{2}+\left(\frac{w_{i}-w(\mathrm{I}, Z, t, \psi)}{\sigma_{w_{i}}}\right)^{2}+\left(\frac{\delta_{i}-\delta(\mathrm{\Gamma}, Z, t, \psi)}{\sigma_{\delta_{i}}}\right)^{2}
$$

where $\delta_{i}$ and $\sigma_{\delta_{i}}$ are the $\mathrm{H} \delta_{A}$ value and error of the $i$ th galaxy. The value of $\chi_{i}^{2}(\Gamma)$ for each galaxy is again determined by equation $2.10 . \mathrm{H} \delta_{A}$ values are simulated using the observed 
values and errors.

The results of the three parameter analysis for $100 \mathrm{MC}$ simulations are shown in black in Figure 3.22. For reference the results of the two parameter analysis of Figure 3.2 are plotted in red. The upper panel shows that for galaxies fainter than $M_{r, 0.1}=-21$ the IMF results for the two and three parameter analyses are nearly indistinguishable. The two analyses yield different results for galaxies brighter than $\mathrm{M}_{r, 0.1}=-21$. In the two parameter analysis the galaxies on the bright end favor increasingly large values of $\Gamma$, while in the three parameter analysis the luminous galaxies all fit the same IMF which is roughly $\Gamma \sim 1.38$.

In the lower panel the $\overline{\chi^{2}}$ values are shown for both analyses. Overall they are qualitatively similar. In both cases $\overline{\chi^{2}}$ is smaller for luminous galaxies and steadily increases as luminosity decreases. However this effect is much more pronounced in the two parameter analysis. At all luminosities the $\overline{\chi^{2}}$ values are significantly higher for the three parameter analysis than the two parameter analysis. All things being equal it might be naively expected that the $\overline{\chi^{2}}$ values should increase by $50 \%$ with the addition of a third parameter. This is approximately the case at the low luminosity end, but for the most luminous galaxies the $\overline{\chi^{2}}$ are increased by more than a factor of 10 .

The effect of the addition of the $\mathrm{H} \delta_{A}$ values on the $\Gamma$ values for the luminous galaxies can be understood by a closer look at several of the earlier figures. In Figure 3.14 it can be seen that the luminous galaxies tend to be redder and have lower EWW. Figure 2.9 reveals that this is precisely the region of color-EW space where the IMF models are most degenerate. For the reddest galaxies that color and $\mathrm{H} \alpha \mathrm{EW}$ do not constrain the 
IMF at all. Even so for a given point in the color-EW plane a particular IMF will only fit for a narrow range of age, metallicity and SFH. Figure 3.20 shows that $\mathrm{H} \delta_{A}$ is acutely sensitive to age, and for ages greater than a few Gyr has a strong functional dependence on SFH and metallicity as well. By providing extra information about the age, metallicity, and $\mathrm{SFH}$, albeit in an apparently convoluted mixture, the $\mathrm{H} \delta_{A}$ values allow the $\chi^{2}$ analysis to do a better job of picking out a best $\Gamma$ from choices that appear less distinct in the two parameter analysis.

Figures 2.8 and 2.9 show that the IMF degeneracies with age, metallicity and SFH are the smallest for the bluest galaxies. Thus for bluer galaxies the $H \delta_{\Lambda}$ values provide less useful new information in the context of measuring the IMF. As Figure 3.14 shows the faintest galaxies tend to be bluer. As a group the $\mathrm{H} \delta_{A}$ values have little effect on the measured $\Gamma$ value which is borne out in Figure 3.22.

The conclusions from the three parameter MC analysis are similar to those of the two parameter analysis with one major improvement. On the faint end galaxies are still a poor fit to a single universal IMF. In addition they are better fit by steeper IMFs. On the bright end galaxies are better represented by a universal IMF with smooth SFHs. The major improvement is that the IMF which best fits the bright galaxies is not only universal at a particular luminosity, but universal for all galaxies brighter than $\mathrm{M}_{r, 0.1}=-21$. Even better is that this universal IMF value is $\Gamma \sim 1.38$ which is in excellent agreement with a wide range of earlier studies suggesting a universal Salpeter slope of $\Gamma=1.35$. 


\subsection{Conclusions}

The goal of this paper was to revisit the K83 method for inferring the IMF from integrated stellar populations and to harness the richness of the SDSS data, improved spectral synthesis models and greater computational power available today to make a stateof-the-art measurement of the IMF. The quality of the SDSS spectroscopy allowed us to address several of the limitations of K83 and KTC94- we resolve the [N II] lines (a significant improvement in the accuracy of the $\mathrm{H} \alpha$ EWs of individual galaxies), eliminate contamination from AGN, make extinction corrections for individual galaxies and fit underlying stellar absorption of $\mathrm{H} \alpha$. We succeeded in achieving more accurate EWs for individual galaxies. The median total EW error for our sample is $17 \%$ compared to a $10 \%$ uncertainty in EWs combined with a $20-30 \%$ uncertainty in the extinction correction for K83.

We expanded the grid of models to allow for a range of ages and metallicities. We used $\chi^{2}$ minimization to go beyond differentiating between two or three IMF models to actual fitting for the best IMF slope. The vast size of the SDSS sample allowed us to both drive down random errors and to cut the data into narrow parameter ranges which were still statistically viable.

The size of the DR4 sample yielded $\Delta \Gamma=0.001195 \%$ confidence region due to random error for the sample as a whole. Even the volume limited $\mathrm{M}_{r, 0.1}=-17$ luminosity bin with only 329 objects has a random error of $\Delta \Gamma=0.0086$. Only in bins with fewer than 10 objects do the random errors become significant. Our IMF fitting is therefore dominated by systematics.

Originally we believed that our systematics would be dominated by the effects of 
SFH discontinuities. However we conducted several experiments where we selected popnlations of galaxies from models with bursting or gasping SFHs and gave them measurement; errors consistent with those in the $\mathrm{M}_{r, 0.1}=-17$ luminosity bin. To our surprise our $\chi^{2}$ minimization revealed the true IMFs with $\Delta \Gamma=0.01$. The main effect was to reduce the quality of the fits. This is due to the fact that $\mathrm{H} \alpha$ EWs return to nominal levels in a relatively short time after SFH discontinuities.

Another way to estimate the size of the systematic errors is to look at the trends of the $\mathrm{M}_{r, 0.1}=-21$ and -22 luminosity bins, because they have the largest membership, in Figures 3.6, 3.7 and 3.8. Assuming that the IMF is universal and that our method is perfect we should get the same answer for any subset of the data we might choose. The largest ranges are $\Delta \Gamma \sim 0.12$ for redshift binning, $\Delta \Gamma \sim 0.19$ by aperture and $\Delta \Gamma \sim 0.19$ by extinction. Conservatively then the systematic error is \pm 0.1 .

There are two points to be kept in mind about this estimate of the systematic error. For one it is the systematic error in the exact value of $\Gamma$. Even in Figure 3.9 where more narrow bands of measured extinction and aperture fraction are considered the same trends with luminosity are seen as with the sample as a whole. The relative systematics between luminosity bins in these narrow slices is much smaller. The second thing to remember is the way in which we empirically defined our systematic error discounts the possibility of IMF variations. What we have called systematics could actually be science. If galaxies have radial IMF gradients or if dust content plays a strong role in star formation the systematic error could be much smaller. The main area in which we were unable to improve upon the K83 and KTC94 studies is that they were able to match the aperture size to the galaxies 
which avoids the issue of aperture effects.

Another source of systematic error, which was briefly mentioned earlier, is the uncertainties in the spectral synthesis models. The level of these errors is difficult to quantify due the complexity of the codes. In addition, a lack of observational data for some regions of parameter space hinders a clear understanding of the full range of variations in real stellar populations.

Cerviño \&Luridiana (2005) give a review of the uncertainties in spectral synthesis models. They divide the main sources of uncertainty into five groups. The first are uncertainties related to the evolutionary tracks and isochrones. These problems arise from the interpolation schemes. One key problem is that of short lived evolutionary phases. For post main sequence stars the relationship between mass and luminosity is not always welldefined. These phases are difficult to handle because small changes in the initial mass of a star can have a profound effect on the luminosity.

Secondly there are uncertainties in the stellar models used. Stellar atmosphere models are usually given by a coarse grid of surface gravities and effective temperatures. However the isochrones used in spectral synthesis models are continuous in these parameters. As such an interpolation scheme needs to be used.

Thirdly they list uncertainties in the assumed stellar birth rate. This is not a problem here, a wide range of SFHs and IMFs are considered. The fourth problem is errors introduced by the codes themselves, either due to bugs or insufficient numerical precision.

The last group of problems deals with gaps in the the evolutionary tracks and stellar atmosphere models and insufficient sampling of the evolutionary phases. To cover 
the full range of parameters of AGB stars the evolutionary tracks may come from different authors. As a result they may not be consistent with each other. Ad hoc assumptions are made to connect the AGB and post-AGB tracks as well. Another problem is the large mass rates experienced in massive star evolution. Cerviño \&Luridiana (2005) do not attempt to quantify the level of uncertainty introduced by these problems given the lack of a clear physical picture of these evolutionary phases to use as a point of reference.

One simple way to test the level of systematic errors introduced by the spectral synthesis models is to compare the output of multiple, independently made codes. Like PEGASE, Bruzual \& Charlot (2003) (hereafter BC03) provide a publicly available spectral synthesis code which uses a different set of stellar atmosphere models. Le Borgne, et al. (2004) compares the outputs of the PEGASE and BC03 models. They find that the Lick indices for Balmer lines and metallic lines are in good agreement between PEGASE and BC03 except for old stellar populations with super-solar metallicities. They suggest the source of this problem is due to incompleteness of the PEGASE stellar library, which has very few cool, high metallicity giant stars. This does not seem to be a problem for this project as the deviations from a universal IMF happen in faint galaxies where the metallicities are low. In addition models were calculated using PEGASE to match those in K83 which were created with another independent code. Again, the PEGASE and K83 models were in good agreement.

Spectral synthesis models have proven themselves extremely useful for the interpretation of galaxy spectra starting from the seminal work of Tinsley \& Gunn (1976). The output spectra are found to be in excellent agreement with observed spectra (e.g. Brinch- 
mann, et al. (2004)). In addition the high luminosity galaxies in this analysis are woll represented by the models. As such the uncertainties in spectral synthesis models are far from catastrophic for general purposes. However given the large sample size in this project the random errors in this analysis are negligible and as a result small systematic errors can potentially have a strong influence on the interpretation of the results. In order to definitively say that the results here are due to real variations in the IMF a better understanding of systematic uncertainties introduced by stellar models is needed. Unfortunately this is a very challenging topic to address.

In spite of a more quantitative approach, like K83 and KTC94 the results are mostly qualitative. However there are four key results from our investigation.

First, for galaxies brighter than $\mathrm{M}_{r, 0.1} \sim-20$ the best fitting IMFs are Salpeterlike $(\Gamma \sim 1.4)$. In addition the assumption of a universal IMF and smoothly varying SFHs is a good fit. This is reassuring as it follows the conventional wisdom and provides confidence that the method works.

Secondly, galaxies fainter than $\mathrm{M}_{r, 0.1} \sim-20$ are best fit by steeper IMFs with larger fractions of low mass stars. For these galaxies a universal IMF and smooth SFH is a poor assumption. This result is in qualitative agreement with evidence that LSBs have bottom-heavy IMFs (Lee, et al. 2004).

Thirdly, while breaking the IMF-SFH degeneracy for individual galaxies using the HIx EW and color is hopeless, for a statistical sample of galaxies the degeneracy can be broken.

Lastly, given our analysis of discontinuous SFHs it appears that the IMF is not 
universal in low luminosity galaxies and fewer massive stars are being created in these galaxies.

It is worth mentioning the main caveat of our $\Gamma$ values again. As illustrated in Figure 2.7 IMF parameterizations are themselves degenerate in our parameter space. Increasing the IMF slope has a similar effect to lowering the highest mass stars that are formed or increasing the fraction of intermediate mass stars. This method cannot explicitly determine if two populations have the same underlying IMF. Figure 3.1 shows that for the sample as a whole the Scalo (1998) three part power law yields nearly the same result as our two part power law. However our method is sensitive in many cases if the IMFs are different.

In terms of star forming cloud temperatures the harsher ambient radiation and larger number of sources of cosmic rays present in more luminous galaxies agree qualitatively with our results. With the extra energy hitting the star forming clouds larger masses may be needed for contraction and fractionization may end sooner, suppressing the formation of less massive stars (Larson 1998). Cedrés, Cepa, \& Tomita (2005) find that while the H II regions of the luminous grand design spiral NGC 5457 (M 31) can be reproduced by a single Salpeter IMF, for the low luminosity flocculent galaxy NGC 4395 a blend of two IMFs is required. However, such trends are not seen in studies of well-resolved stellar populations (Kroupa 2002).

Anohler explanation for the absence of massive stars is that the massive stars are there, but are not visible. Extinction to the center of star forming regions, where massive stars preferentially exist, can reach $A_{V} \sim 20$ (Engelbracht, et al. 1998). However the low 
luminosity galaxies have the lowest observed extinctions (sce Table 3.1) which is the opposite of what would be expected given our IMF results.

It is also possible that the IMF is in fact universal, but the way in which it is sampled in embedded star clusters leads to an integrated galaxial IMF which varies from the true IMF. Weidner \& Kroupa (2005) use a universal IMF with the assumption that stars are born in clusters where the maximum cluster mass is related to the star formation rate. For a range of models this leads to a narrow range for the apparent IMF in high mass galaxies. For low mass galaxies the IMF is steeper with a wider range of slopes. The results here are in qualitative agreement for some of the integrated galactic IMF scenarios in Weidner \& Kroupa (2005) given that there should be a rough correlation between galaxy luminosity and mass. Once again, Elmegreen (2006) argues that the galaxy wide IMF should be the same as the IMF in clusters regardless.

In light of the theory of Weidner \& Kroupa (2005), whether the results of this paper speak to a relationship between environment and the formation of individual stars is open to interpretation. However the impact on the modeling and interpretation of the properties of galaxies is clear. Köppen, Weidner, \& Kroupa (2007) note that the integrated galaxial IMF is the correct IMF to use when studying global properties of galaxies. Even if the IMF of stars is in truth universal it may currently be misused in the modeling of galaxies. Furthermore a varying integrated galaxial IMF could open the door to new insights in galaxy cvolution. For instance, Köppen, Weidner, \& Kroupa (2007) suggest that the observed mass-metallicity relationship in galaxies naturally arises from a variable integrated galaxial IMF similar to the results of this paper. 


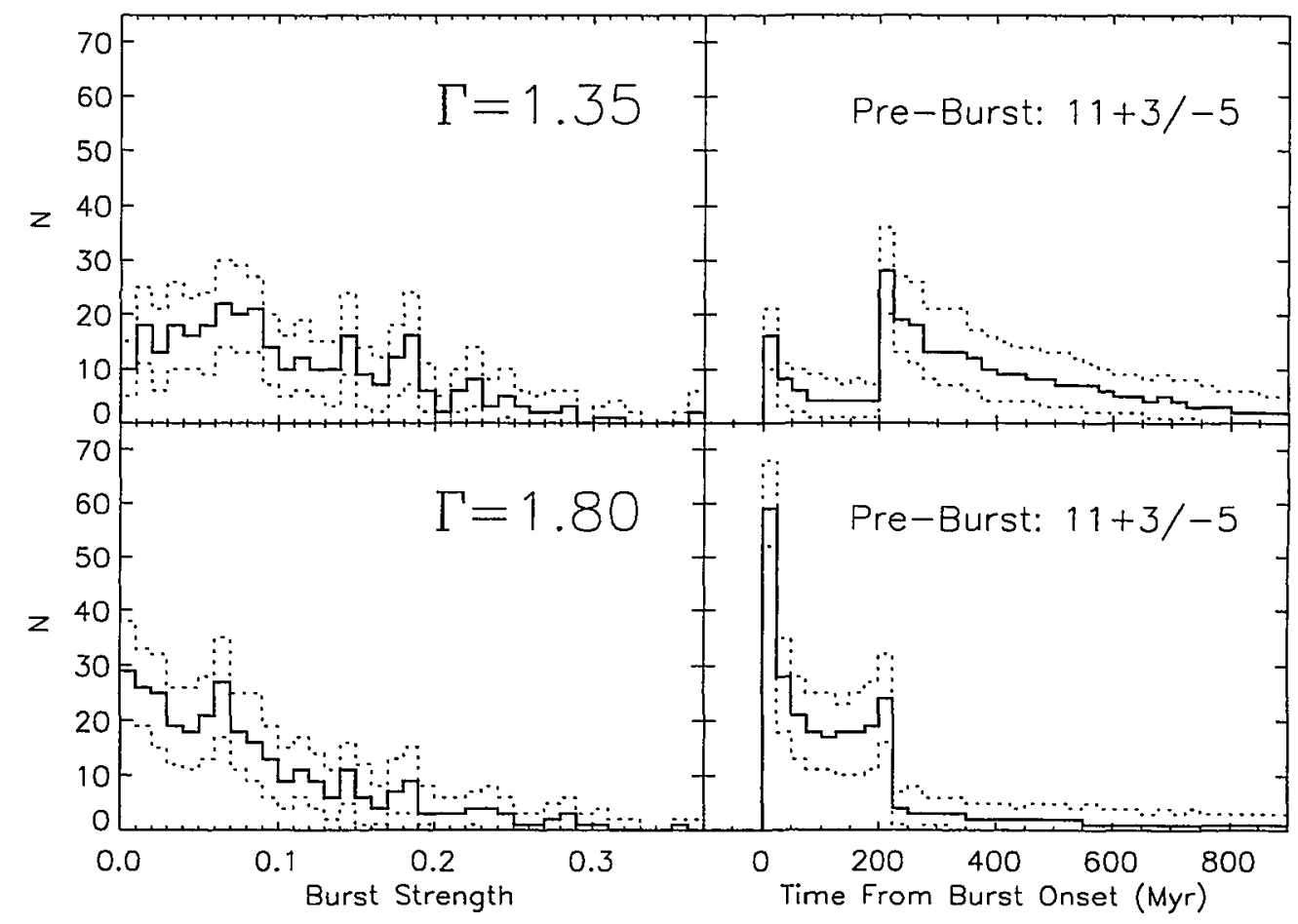

Figure 3.17 Distributions of best fitting parameters for the synthetic $\mathrm{M}_{r, 0.1}=-17$ data for models with $Z=0.01$ and a single burst SFH. In all panels the dotted lines indicate the, $95 \%$ errors determined from MC simulations. In the top two panels are results for $\Gamma=1.35$. In the bottom two panels are results for $\Gamma=1.80$. In the left column is the distribution of best fitting burst strengths given as a fraction of the total stellar mass formed. At right is the distribution of best fitting ages measured relative to the onset of the bursts. The bursts of star formation begin at $U$ and end at $200 \mathrm{Myr}$. For both IMFs $11+3 /-5$ of the 329 galaxies are best fit by models which have yet to experience their bursts and are not plotted. 


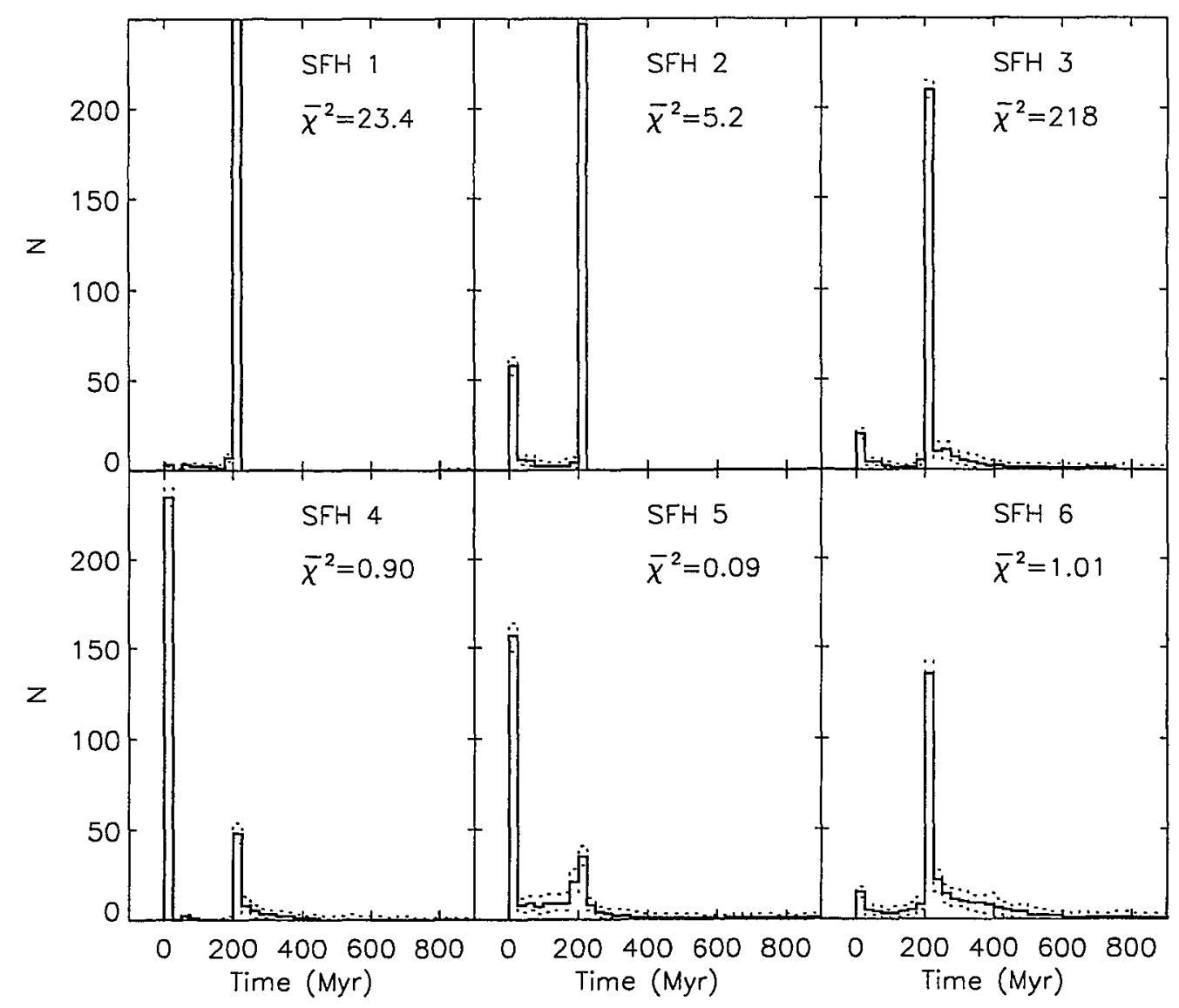

Figure 3.18 Distributions of best fitting times measured from the beginning of a burst or gasp for the synthetic $\mathrm{M}_{r, 0.1}=-17$ data for models with $Z=0.01, \Gamma=1.35$ and multiple burst SFIs models described in Table 3.2 . The $\overline{\chi^{2}}$ values for each family of models is also shown. The dotted lines indicate the $95 \%$ confidence regions determined by MC simulations. 


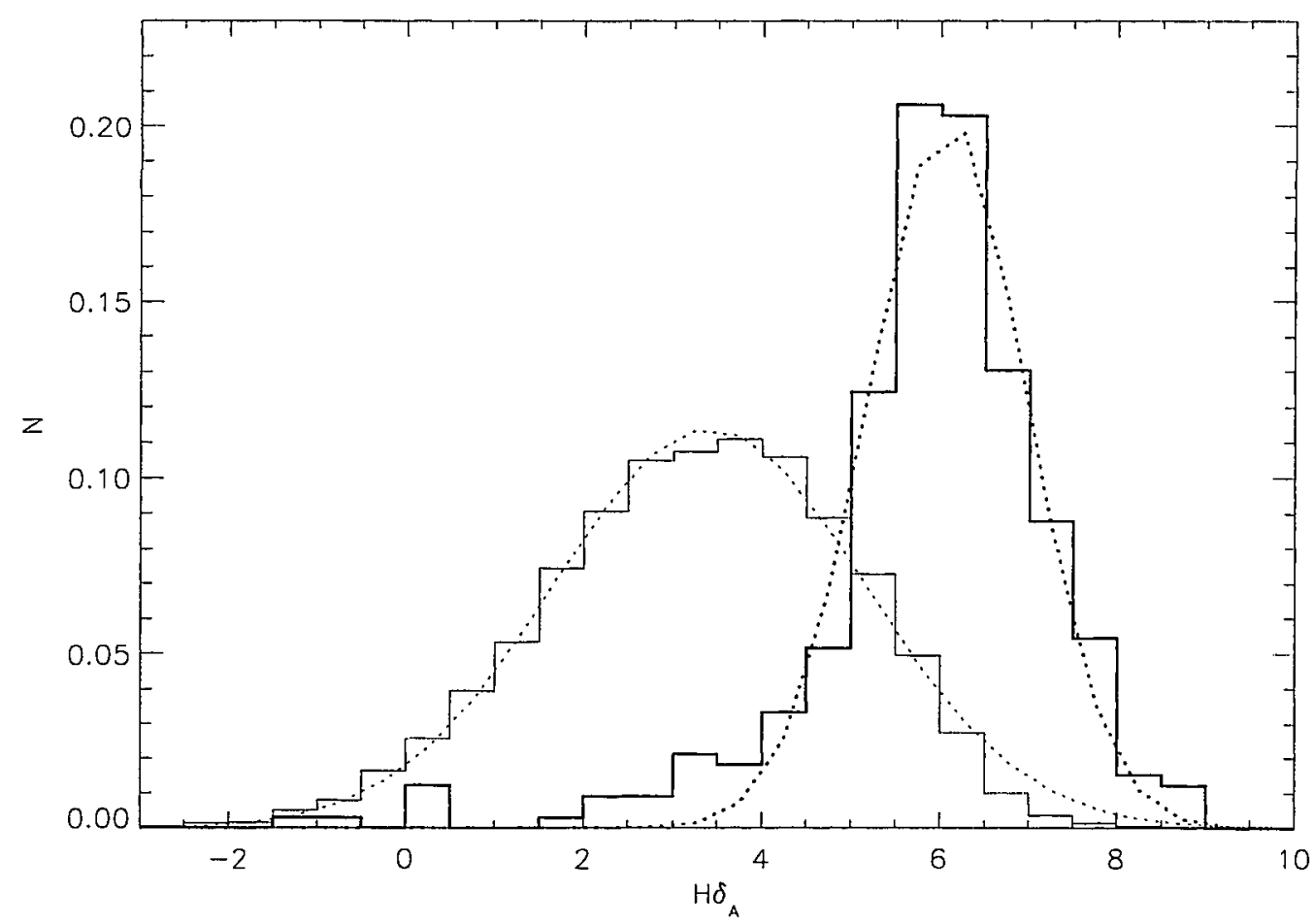

Figure 3.19 Histogram of the measured $\mathrm{H} \delta_{\mathrm{A}}$ values for the $\mathrm{M}_{r, 0.1}=-17$ (bold line) and $\mathrm{M}_{r, 0.1}=-23$ (thin line) volume limited bins from Figure 3.14 expressed as a fraction of the total number of galaxies in each luminosity bin. The median uncertainty in $\mathrm{H} \delta_{\mathrm{A}}$ is 0.9 in both bins. The dotted lines are Gaussian profiles fitted to the distributions. For the $\mathrm{M}_{r, 0.1}=-23$ bin (thin dots) the profile is centered on 3.4 with $\sigma=1.8$ and for $\mathrm{M}_{r, 0.1}=-17$ bin (thick dots) the profile is centered on 6.1 with $\sigma=0.9$. 


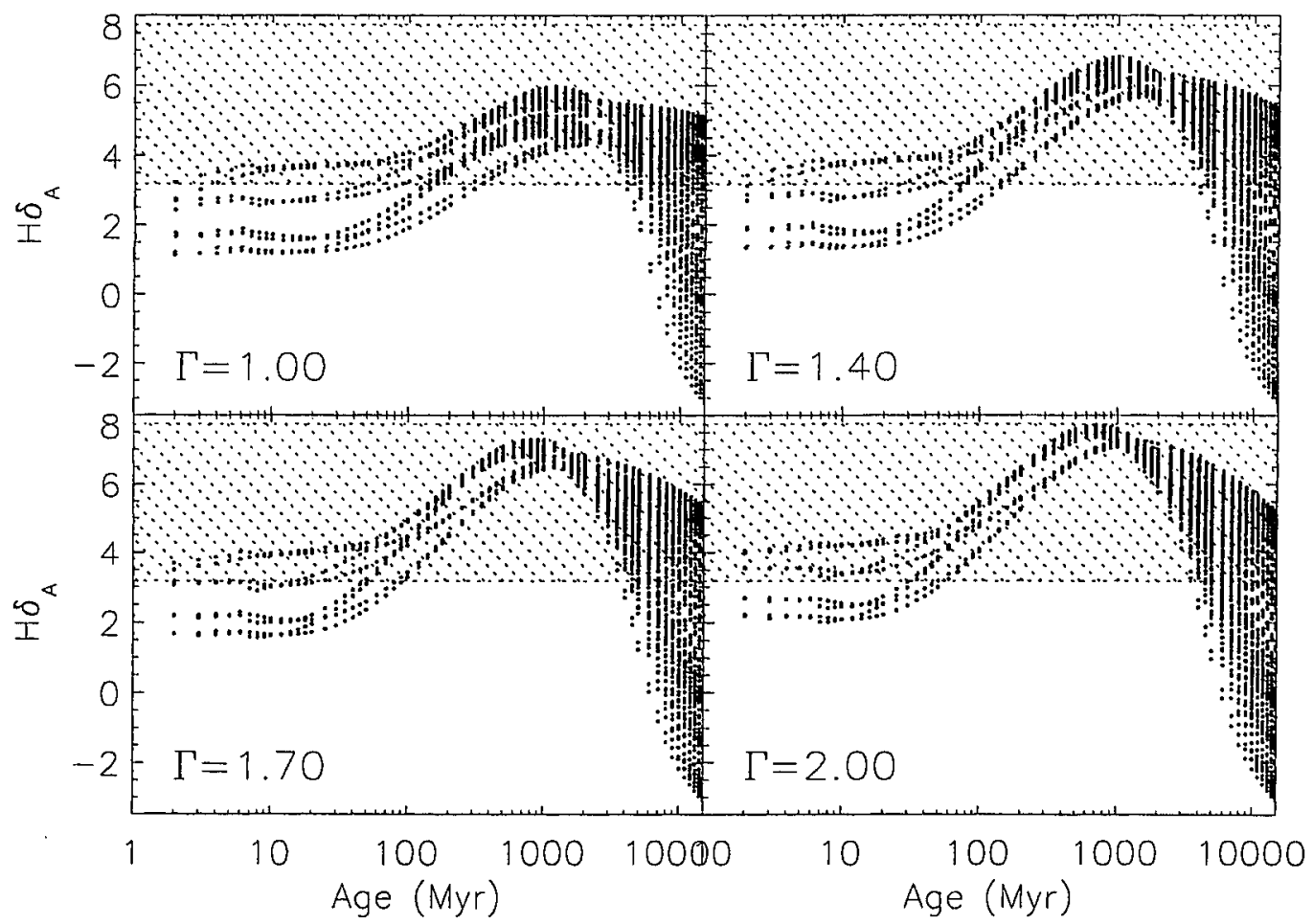

Figure 3.20 Model values of the $\mathrm{H} \delta_{\mathrm{A}}$ index as a function of age for four IMF models, $\Gamma=1.00$ (upper left), 1.40 (upper right), 1.70 (lower left), and 2.00 (lower right). Each dot represents a model value for single IMF, metallicity, SFH (as described in the models section) and age. The four tracks identifiable towards the left of each panel are each of differing metallicities with the lowest metallicity models having the largest $\mathrm{H} \delta_{\mathrm{A}}$ values at young ages. The grey area indicates the range of $H \delta_{\mathrm{A}}$ spanned by the middle $90 \%$ of the volume limited $\mathrm{M}_{r, 0.1}=-23$ bin. The hashed area shows the middle $90 \%$ range for the volume limited $\mathrm{M}_{r, 0.1}=-17$ bin. 


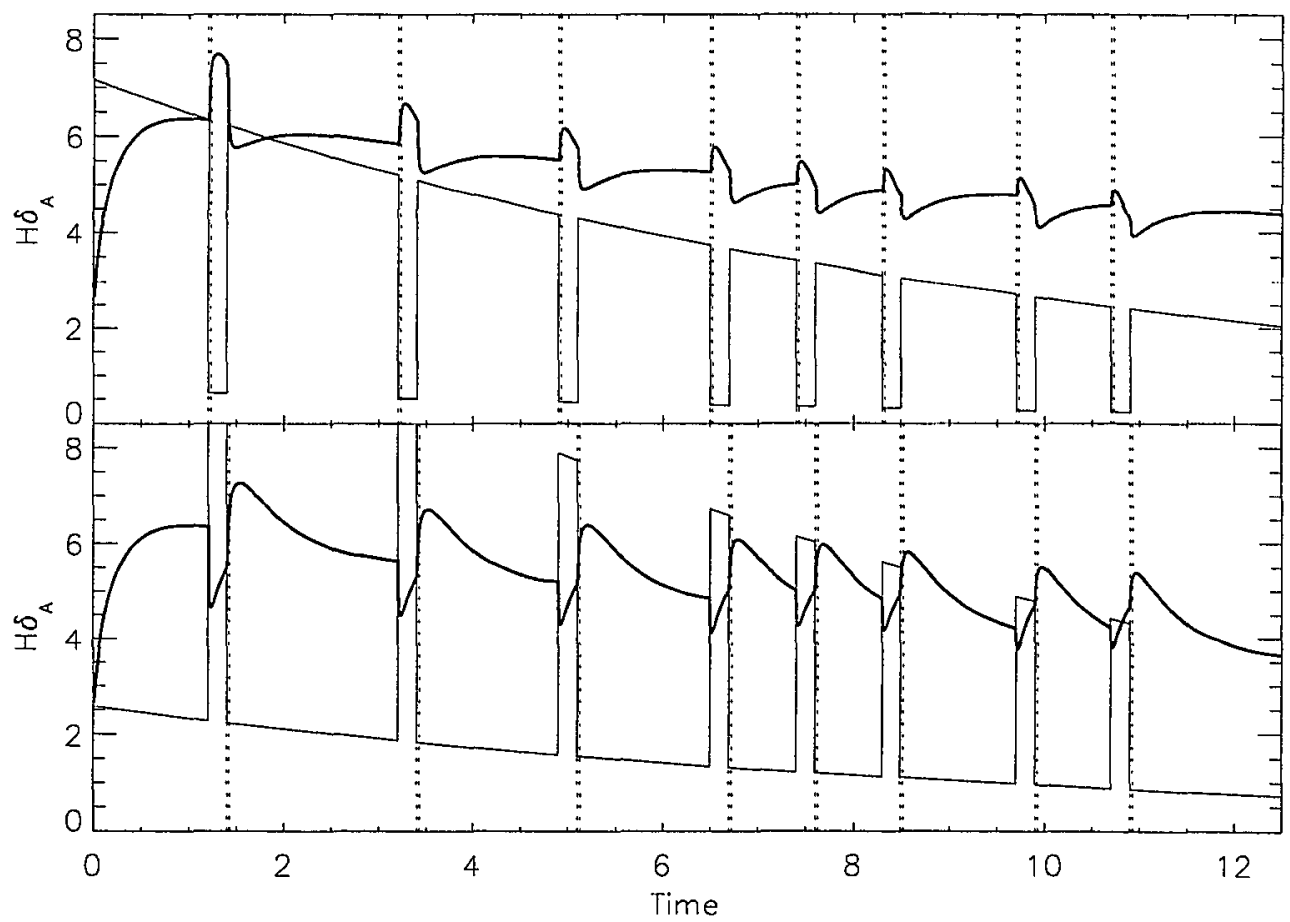

Figure 3.21 Model $\mathrm{H} \delta_{\mathrm{A}}$ values as a function of age for two multiple burst models. The $\mathrm{H} \delta_{\mathrm{A}}$ values are denoted by the bold line. The SFHs (thin line) are overplotted with arbitrary normalization for reference. The underlying exponentially decaying SFH is the same in both panels. The top panel has superposed gasps of star formation (SFH \#5) while in the bottom panel has bursts (SFH \#6). The vertical dotted lines mark the 20 Myr intervals after a $\mathrm{SFH}$ discontinuity which best fit the distribution of colors and $\mathrm{H} \alpha \mathrm{EWs}$ in the $\mathrm{M}_{r, 0.1}=-17$ luminosity bin. 


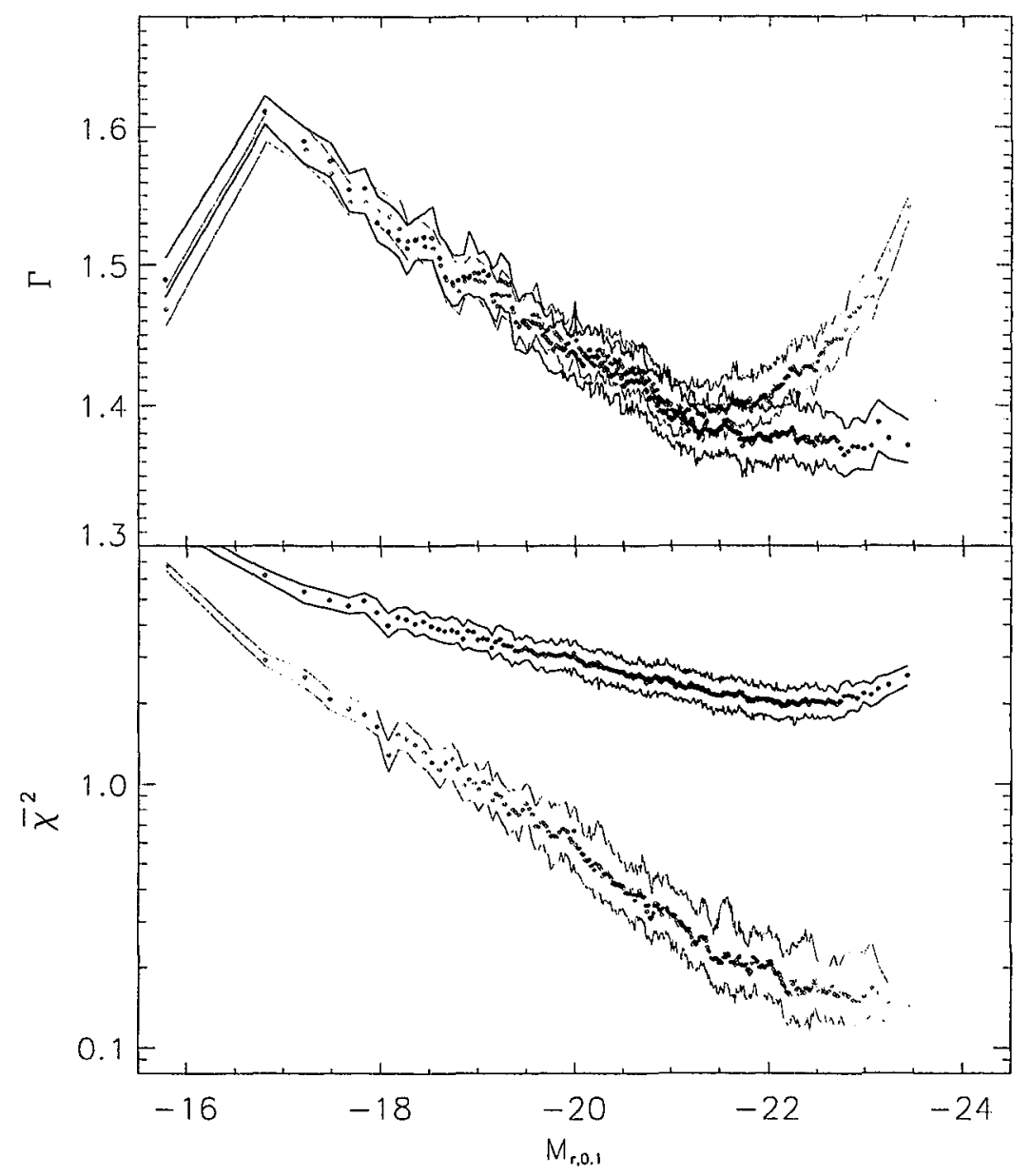

Figure 3.22 $\mathrm{MC}$ simulation results for the full sample binned by $\mathrm{M}_{r, 0.1}$. Each diamond represents 500 galaxies plotted at the mean $\mathrm{M}_{r, 0.1}$ value of the bin. The solid lines represent the upper and lower $95 \%$ confidence region measured for each bin. The three parameter results, including the $\mathrm{H} \delta$ absorption data, are plotted in black. The two parameter results from Figure 3.2 are plotted in red for reference. Top panel: The best fitting $\Gamma$ values as a function of $r$-band luminosity. Lower panel: The $\overline{\chi^{2}}$ values for each luminosity plotted on a log scale. 


\section{Chapter 4}

\section{Photometric Algorithms for Selecting Lensed Lyman Break Galaxies}

In $\S 1.7$ the power of the Lyman break technique for identifying a population of galaxies in the high redshift universe was discussed. This technique has provided a wealth of information on the precursors to today's elliptical galaxies and spiral bulges filling in gaps in our understanding of the evolution of galaxies. Given the intrinsic faintness of LBGs due to their large distances in general they can only be studied as a population due to difficulties in observing them. However $\S 1.7$ also detailed the discovery of a handful of LBGs which have been magnified by intervening galaxy clusters by gravitational lensing. This marriage of general relativity and a fortuitous geometric alignment of astrophysical objects allows for the in depth study of individual high redshift galaxics.

The known strongly lensed LBGs in $\S 1.7$ have in common that they were all found serendipitously, primarily in studies of galaxy clusters. However the treasure trove of information concealed in each of these objects suggests that they deserve a search of their 
own. In this chapter a targeted, photometric search for strongly lensed LBGs is described, as well a few peripheral projects using different techniques dedicated to the same goal of uncovering lensed LBGs.

\subsection{LBGs in Color Space}

In order to design a targeted, photometric search for LBGs the first step is to know what one is looking for. In this section the expected photometric properties of LABGs are discussed.

Figure 4.3 shows a model LBG spectrum from the Lyman continuum to the nearinfrared at $1 \mu \mathrm{m}$. The spectrum is that of a young stellar population with strong star formation. The most important feature of the spectrum as far as this project is concerned is the absence of flux below the Lyman limit at $912 \AA$.

Figures $4.4,4.5$, and 4.6 show the path through color space taken by LBGs. This is accomplished by taking the LBG model spectrum of Figure 4.3, combining it with an model of extinction from the intergalactic medium and incrementally redshifting the spectrum while convolving it with the SDSS filter responses. Just as importantly for context the stellar locus and low redshift galaxy model tracks are plotted.

The black dots in the figure are actual point sources selected from a contiguous 20 $\operatorname{deg}^{2}$ arbitrarily chosen region along the celestial equator. The SDSS query selected all point sources in the region with $15<r<18$ in PSF magnitudes. This yielded 16,429 objects. In order to distinctly show the stellar locus objects with potentially spurious photometry were rejected with a flag filter. Objects with one or more of the CHILD, CR, or INTERP flags 
set by the pipeline were rejected leaving 6,310 objects. The black dots show the location of the stellar locus with what is likely a small contamination of a few quasars.

The blue lines are model tracks of elliptical/SO, Sb/Sc and Sd/Irr galaxies for $0<z<1$. Each track starts on the stellar locus at $z=0$ and then deviates off of it as the redshift increases. As galaxies appear as the luminosity weighted mean of their stellar populations it is not surprising that they should have colors similar to those of stars. The tracks spiral off the stellar locus because as the galaxy spectra are redshifted the rest frame wavelengths falling into the filters on Earth no longer match those of the stars. With their old, red and dead populations the elliptical templates are the reddest of all templates in each color with the Sd/Irr templates being the bluest.

These figures vividly illustrate the key trick to the Lyman break technique; LBGs at high redshifts $z>2$ occupy regions of color space which distinguish them from objects within the Galaxy and in the local universe. The LBG tracks in the figures are color coded to show the different redshift regions of interest. The LBG track is plotted in blue for $0<z<1$ just as the other galaxy templates. Were there any nearby LBGs they would appear even bluer than Sd/Irr galaxies on account of their intense star formation and young stellar populations. Eventually the redshift reaches a point where the $912 \AA$ Lyman continuum limit starts to pass through the telescope filters. The cyan line shows LBGs for which $1<z<2.29$, beyond $z=1$ but before the Lyman break reaches the $u$ band filter. The algorithns here are unlikely to be able to identify LBGs with $z<2.29$. However, were the SDSS data combined with ultraviolet data lower redshifts could be accessed.

The green line in the figures shows the location in color space of the $u$-band 
dropouts - galaxies at redshifts for which the Lyman break falls in the $u$ filter. This occurs for redshifts in the range of $2.29<z<3.17$. As the break passes through the $u$ filter the $u$-band flux quickly decreases to zero. As evidenced in Figure 4.4 the galaxies quickly become very red in the $u-g$ color. A similar scenario plays out for the gband dropouts, $3.17<z<4.92$, shown in orange and the $r$-band dropouts, $4.92<z<6.34$, shown in red.

Given the clear separation of LBGs from other objects in Figures 4.4, 4.5, and 4.6 it appears that finding strongly lensed LBGs in the SDSS should be trivial. However that is not the case in practice. As objects become fainter the photometry errors become increasing larger. This is illustrated in Figure 4.7.

For comparison the first column of Figure 4.7 are copies of Figures 4.4, 4.5, and 4.6 where the labels have been omitted for clarity and where the density of points is high they have been replaced by logarithmic contours. The first column shows 6,310 point sources with $15<r<18$ which survived the previously described photometry flag filter. In the second column the distribution of fainter point sources is shown in $u-g$ vs. $g-r, g-r$ vs. $r-i$, and $r-i$ vs. $i-z$ color space from top to bottom. These objects come from the same $20 \mathrm{deg}^{2}$ region on the sky and survive the same flag filter but have $18<r<21$. In this magnitude bin there are 29,007 point sources, more than 4.5 times as many in the first column. The brighter objects sharply delineate the stelar locus. However the decreasing photometry quality in the fainter objects leads to much more scatter in the stellar locus.

This illuminates one of the challenges in this project. As fainter LBGs are sought not only does their photometry become more uncertain, but the stellar locus simultaneously becomes more poorly defined. In particular in the $u-g$ vs. $g-r$ color space at top middle 
the distribution becomes spread out horizontally for large values of $g-r$. For particularly red objects the $u$ magnitude is considerably fainter than the $r$ magnitude. The problem is compounded by the fact that the system response is much weaker in the $u$ band than the $r$ band. Thus for an $r$ band selected sample the $u$ band contains many weak or non-detections for the reddest objects. As a result the $u-g$ color for these objects is largely meaningless. Because of the luptitude system all objects have magnitudes in all bands. As shown in Table 4.10 flux in the $u$-band leads to a magnitude of 24.63 but given flux errors can be higher or lower by more than a magnitude. This yields an arbitrary $u-g$ color as seen in the figure.

The third column of Figure 4.7 shows the distribution of the 28,988 extended objects surviving the flag filter in the same $20 \mathrm{deg}^{2}$ region in the $18<r<21$ magnitude range. When selecting the galaxies the model magnitudes, which is the optimal system for faint galaxies, were used rather than the PSF magnitudes used for point sources. In the third column the true challenge of this project becomes apparent. While the densest regions of objects agree well with the low redshift galaxy tracks there is a large amount of scatter in color space. One would naively hope that a clear population would be evident along the LBG track lines which could easily be targeted. This is clearly not the case. Furthermore there are no clear edges to the galactic locus but rather a smoothly decreasing density of points extending outward from the locus. The problem is particularly bad in the $g-r$ vs. $r-i$ color space where the spread of the galactic locus fully overlaps the LGB track. Using these two colors along it is practically impossible to target LBGs given the quality of the photometry. 
The challenge of the project is to use as much information in the SDSS as possible to eliminate stellar and galactic contaminants without throwing out real LBGs. Even so, given the size of the photometry errors the sheer number of objects in the photometric: catalog there will be several objects whose erroneous photometry mimics that of LBGs regardless of their underlying nature.

\subsection{Ins and Outs of the SDSS Data}

The SDSS is comprised of photometric and spectroscopic data which is in many ways conventional and very familiar to optical astronomers. However there are some subtleties which have been developed by or are unique to the SDSS. Knowledge of these is essential to understanding the SDSS data and being able to deal with the huge quantity of data in the survey in a sensible way. In this section relevant survey nuances are discussed.

\subsubsection{SDSS Magnitude Systems}

Magnitudes in the SDSS are measured using a modified system of inverse hyperbolic sine magnitudes, or luptitudes (Lupton, Gunn \& Szalay 1999). For bright objects luptitudes assymptotically approach $A B$ magnitudes and the difference between them is academic. The difference lies at faint magnitudes and is motivated by a desire to preserve as much information as possible from the photometry.

For example, due to Poisson noise measuring the flux of a drop out galaxy in a sky subtracted image in the bandpass it has dropped out of will yield a positive flux $50 \%$ of the time and a negative flux the other $50 \%$. Because conventional magnitudes are logarithms 
of the flux they are undefined for negative fluxes and quickly approach erroneously large magnitudes for very small positive fluxes. In many surveys objects with problematic photometry, regardless of the reason, are assigned a magnitude of -99 or some other fixed value. This solution makes the recovery of fluxes impossible, destroying important information. If the magnitude is undefined because the flux is small and negative it is consistent with a dropout galaxy. However, if the flux is large and negative it may indicate that there is a problem with the sky subtraction and the galaxy which appears from the photometry to be a dropout could be something more mundane.

Luptitudes are well behaved for any value of the flux. They are defined by

$$
\mu=-a\left[\sinh ^{-1}\left(\frac{f / f_{0}}{2 b}\right)+\ln b\right]
$$

where $\mu$ is the luptitude and $a=2.5 \log _{10} e=1.08574$ is Pogson's ratio. $b$ is called the softening parameter. It determines the flux at whichthe behavior becomes linear. The inverse hyperbolic sine is defined by:

$$
\sinh ^{-1}(x)=\ln \left[x+\sqrt{x^{2}+1}\right]
$$

The softening parameter is usually the $1 \sigma$ noise of the sky in a PSF aperature with 1" seeing.

There are a number of reasons why it is useful to convert to flux or magnitudes. Using the fluxes and filter centers one can construct a spectral energy distribution for any object. This can be used to compare to template spectra. When determining how likely an object is undetected in a particular band it is more intuitive to speak in terms of flux. The flux and flux error can be determined by the following equations, where $\sigma_{\mu}$ and $\sigma_{f}$ are the 
Table 4.1. SDSS Softening Parameter and Limiting Magnitudes

\begin{tabular}{cccccccc}
\hline \hline Filter & $b$ & $m_{0}$ & $1 \sigma$ & $3 \sigma$ & $5 \sigma$ & $10 \sigma$ & $95 \%$ \\
\hline & & & & & & & \\
$u$ & $1.4 \times 10^{-10}$ & 24.63 & 24.11 & 23.34 & 22.85 & 22.12 & 22.0 \\
$g$ & $0.9 \times 10^{-10}$ & 25.11 & 24.59 & 23.82 & 23.33 & 22.60 & 22.2 \\
$r$ & $1.2 \times 10^{-10}$ & 24.80 & 24.28 & 23.50 & 23.01 & 22.29 & 22.2 \\
$i$ & $1.8 \times 10^{-10}$ & 24.36 & 23.84 & 23.06 & 22.57 & 21.85 & 21.3 \\
$z$ & $7.4 \times 10^{-10}$ & 22.83 & 22.30 & 21.53 & 21.04 & 20.32 & 20.5 \\
\hline
\end{tabular}

luptitude and flux errors.

$$
\begin{gathered}
\frac{f}{f_{0}}=2 b \sinh \left[-\left(\frac{\mu}{a}+\ln b\right)\right] \\
\sigma_{f}=\frac{\sigma_{\mu} \sqrt{4 b^{2}+\left(f / f_{0}\right)^{2}}}{a}
\end{gathered}
$$

It may also be helpful to convert the luptitudes to magnitudes, as it is a more familiar way of doing things. The flux ratio, $f / f_{0}$ defined above, is used in the standard magnitude formula found in equation 1.31. The flux error can be converted to the magnitude error using:

$$
\sigma_{m}=\frac{a \sigma_{f}}{f / f_{0}}
$$

The values of the softening parameter for the SDSS ugriz system are given in Table 4.1 (Stoughton, et al. 2002). Also in table 4.1 are the limiting magnitudes and magnitudes of $1,3,5$ and $10 \sigma$ detections. These are calculated from equation 4.1 using $0,1 b, 3 b, 5 b$ and $10 b$ as the values of $f / f_{0}$. The last column of the table lists the $95 \%$ completion limits for point sources in each wave band.

Table 4.1 reveals that the $g$ band reaches the deepest magnitudes. In addition 
the $u, g, r$ and $i$ bands have similar sensitivites but the $z$ band lags behind. The sane trend is seen in the $95 \%$ completion limits for point sources although the $g$ and $r$ bands are equally as deep. In practice however the $95 \%$ limits for point sources are overly optimistic for extended objects. More conservative limits must be imposed when dealing with galaxies.

In addition to the non-standard arcsinh magnitudes there is another important detail to be aware of with the SDSS photomertry. The SDSS photometric pipeline measures four different magnitudes for each object as detailed in the introduction. The magnitude which should be used depends on the particular situation. The LBG search deals with faint galaxies, many of which are near the limits of the survey.

Measuring the fluxes of galaxies is a complicated business. As stars are point sources they are well modeled by the point spread function of the system. For this reason the SDSS PSF magnitudes are the preferred magnitudes for studying stars and other pointlike objects like quasars. However galaxies and other extended objects come in all shapes and sizes. They have different surface brightness profiles. Even worse, there are no clear boundaries or edges for galaxies. The SDSS pipeline takes two approaches to this problem: Petrosian and model magnitudes.

\subsubsection{SDSS Data Flags}

Traditionally in astronomy individual or small groups of astronomers make observations on a handful of nights a year and spend the rest of the year intcrpreting the results. Attention can be paid to individual objects to identify problems like cosmic rays, proximity to bright objects and overlapping objects. However the SDSS observes every clear night and while the small group of observes pay close attention to overall data quality they do not 
in general give individual objects specialized attention. As of the most recent data release (DR6) there are 287 million unique objects and 1,271,680 spectra in the SDSS. It is wholly impossible for an individual to inspect each object to check it for potential problems. As a result it is necessary for the photometric and spectroscopic pipelines to have the intelligence to identify problems independently. By corollary the user must posses the knowledge to interpret and heed the pipeline's warnings.

The BINNED1 flag is set if an object was detected in an unbinned image. It is set. in flags if the object was detected unbinned in one or more of the five bands.

The BLENDED flag is set if an object is determined to be a blend.

The CHILD flag is set if an object is created by the deblender. The deblender can potentially create objects with unusual colors. The deblender has undergone several improvements over the course of the survey and is more reliable than in the past. In early versions of our selection there was heavy contamination by deblended objects. Rejecting these objects greatly reduced the number of contaminants.

The EDGE flag is set if an object is too close to the edge of the chip in a given band. This is set, amongst other reasons, if the object is too close to the edge to measure a radial profile. Because early on we used Petrosian magnitudes, and moved on to fixed aperature magnitudes, we rejected objects with this flag to help ensure that the magnitudes we were using were meaningful and to reduce the chances of finding good colors for the wrong reasons. Objects that are rejected for being on the edge should be reobserved in a different run, at which point they will survive this cut. Therefore little is lost in this requirement, except for duplication of objects. This was a concern in the early days of the survey but is 
no longer a problem given improvements to the database.

The CR flag is set if an object contains cosmic ray pixels in one or more bands. The SATUR flag is set if an object has saturated pixels in at least one band. The software will interpolate pixels in both of these situations.

\subsection{LBG Target Selection Algorithms}

Over the six year course of this project the algorithm for targeting strongly lensed LBGs has evolved. Development of the original algorithm was not done with the benefit of SDSS observations of known lensed LBGs. SDSS observations of cB58 were not available until after the second incarnation of the algorithm. Nonetheless the technique was improved over time with lessons learned from trial and error. In this section the algorithms are described as well as the lessons learned from them and the progression towards better selection methods.

\subsubsection{First Generation Target Selection}

\section{Step 1: Flags}

The first step was to make a cut using the SDSS flags for the objects. Using the flags ficld objects with objectionable characteristics in at least one of the five filters are identified. This takes little computation time; by taking this step first the number of objectis sent through more computationally intensive steps is greatly reduced. The flag filtered here employs six of the photometric flags.

The BINNED1 was required to be set. Objects are that were not an unbinned 
detection in at least one band were rejected. Objects with one or more of the BLENDED, CHILD, EDGE, CR and SATUR flags set were also rejected.

\section{Step 2: Error}

For the selection we the fiber magnitudes were used. The fiber magnitudes are defined as the flux enclosed in an aperature of diameter 3 ". The error in the fiber magnitudes for each band is given in the survey. The errors in the five bands were summed and it was required that the sum be between 0 and 8 . Although 8 is a somewhat arbitrary number it gets rid of objects that have highly uncertain magnitudes, and thus highly uncertain colors. At the same time it is not so stingy that it rejects objects with one bad magnitude. The greater than zero requirement is used to remove objects that have variable or otherwise problematic magnitudes. At the time some of the objects in the survey had magnitude errors of -9999.90 even though by the survey design this was not supposed to happen.

\section{Step 3: LBG Selection}

In the third step objects that were not consistent with the region LBGs occupy in color-color space were rejected. In Figure 4.8 shows the LBG tracks in $u-g$ vs. $g-r$ color space, and also for $g-r$ vs. $r-i$ and $r-i$ vs $i-z$ color spaces. Four tracks based on the model spectrum in Figure 4.3 were used which differed by the amount of extinction due to the intergalactic medium. Tracks with mean, median, $10 \%$ and $90 \%$ levels of IGM extinction were used to construct an LBG area in $g-r$ vs. $r-i$ and $r-i$ vs. $i-z$ color-color space using a ruler. In $g-r$ vs. $r-i$ color space this region is defined by locations which 
satisfy all of the following criteria:

$$
\begin{gathered}
(g-r)>-0.2 \\
(r-i)>-0.2 \\
(r-i)<0.2 \text { OR }(r-i)<0.571(g-r)-0.371
\end{gathered}
$$

Similarly in $r-i$ vs. $i-z$ color space LBG candidates must satisfy all of the following:

$$
\begin{aligned}
(r-i) & >-0.2 \\
(i-z) & >-0.2 \\
(i-z)<0.2 \text { OR }(i-z) & <0.667(r-i)-0.067 \\
(i-z) & <2.7
\end{aligned}
$$

\section{Step 4: Brightness}

It was required that $i<21$. This helps eliminate objects too faint to have believable photometry. Such faint objects could not be readily observable with $2 \mathrm{dF}$ given the parameters of the observing program.

\section{Step 5: Star Removal}

The color space areas defined above overlap the stellar locus. It is then necessary to have some method for removing these stars. This was done by selecting around 50,000 objects from the sxStar class and plotting them in color space. Then, by hand, boxes were defined that enclose the stellar locus in each of the three plots. These regions are shown Figure 4.8 and are defined as follows. 
$u-g$ vs. $g-r$ color space:

$$
\begin{gathered}
(u-g)>-0.6 \\
(u-g)<4.0 \\
(g-r)<1.8 \\
(g-r)<0.467(u-g)+0.680 \\
(g-r)>0.538(u-g)-0.754 \text { AND }(u-g)>1.4 \\
(g-r)>0.400(u-g)-0.760 \text { AND }(u-g)<1.4
\end{gathered}
$$

$g-r$ vs. $r-i$ color space:

$$
\begin{gathered}
(g-r)>-0.8 \\
(g-r)<1.8 \\
(r-i)<0.500(g-r)+0.400 \text { AND }(g-r)<1.2 \\
(r-i)>0.500(g-r)-0.300 \\
(r-i)<2.5 \text { AND }(g-r)>1.2
\end{gathered}
$$

$r-i$ vs. $i-z$ color space:

$$
\begin{gathered}
(r-i)>-0.6 \\
(r-i)<2.5 \\
(i-z)>-0.5 \text { AND }(r-i)<0.4 \\
(i-z)>0.428(r-i)-0.171 \text { AND }(r-i)>0.4 \\
(i-z)<0.4 \text { AND }(r-i)<0.4 \\
(i-z)<0.428\left(r^{\prime}-i^{\prime}\right)+0.229 \text { AND }(r-i)>0.4
\end{gathered}
$$

Objects that are in all three boxes are rejected as stars. LBGs with redshifts below $z<4$ will be in the stellar locus in $r-i$ vs. $i-z$ plots, and those with $z<3$ will be confused 
with the stellar locus in both $r-i$ vs. $i-z$ and $g-r$ vs. $r-i$ plots. Requiring objects to be consistent with the stellar locus in all three plots prevents us from rejecting everything but the highest redshift LBGs.

\section{Step 6: Galaxy Contaminants}

There is also contamination due to galaxies. The method used for removing these objects differs from that for stars. The first step is to remove the $\mathrm{Sb} / \mathrm{Sc}$ galaxies. This is accomplished using theoretical tracks for these galaxies. These tracks are calculated in all four colors for $z=0$ to $z=10$ with increments of 0.01 . For each of these 1,000 points the $\chi^{2}$ value is computed as follows:

$$
\chi^{2}=\sum_{i=0}^{3}\left(\frac{c_{o b s, i}-c_{i}}{\delta c_{o b s, i}}\right)^{2}
$$

where $c_{o b s, i}$ are the observed colors $u-g, g-r, r-i$ and $i-z$ of a galaxy, $c_{i}$ are the colors of the model and $\delta c_{o b s, i}$ are the errors in the observed colors. For an independent variable $X$ with a $\chi^{2}$ distribution with $\nu$ degrees of freedom:

$$
P\left(X \leq \chi_{\alpha, \nu}^{2}\right)=\alpha
$$

where the area under the $\chi^{2}$ curve to the right of $\chi_{\alpha, \nu}^{2}$ is equal to $\alpha$. For $\alpha=0.005$ and $\nu=4, \chi_{0.005,4}^{2}=14.860$. Thus $99.5 \%$ of the $\mathrm{Sb} / \mathrm{Sc}$ galaxies will have $\chi^{2}<14.860$. If one or more of the $1,000 \chi^{2}$ values calculated for an object are less than 14.860 , the object is rejected as an $\mathrm{Sb} / \mathrm{Se}$ galaxy.

The largest disadvantages of this method are that it utilizes for loops in IDL which is highly inefficient, and it is otherwise computationally intensive. However, by making this the last step there are on average three objects per 1,000 left by this stage. Despite the fact 
that this is saved for the last step, it still requires the longest amount of time. It takes on order a day to run through all of the objects, but it is not a prohibitively long time.

The above steps are repeated for theoretical Sd and elliptical/S0 tracks.

\section{Step 7: Final Cuts}

In preparing targets for a $2 \mathrm{dF}$ run on July 18,19 , and 21,2001 , the ten best. candidates were identified for each of the predetermined $2 \mathrm{dF}$ fields. The selection routine returned around 100 objects per field of view so it was necessary to further pare down the candidates. The number was cut roughly in half by requiring $z<20.5$. In addition objects with the most extreme colors were targeted. The first fields had more stringent restrictions than those listed below, but these were eased when the decision was made to test more candidates. Many objects that survived did so in multiple ways. This selection was used to find $u$ dropouts.

$$
u-g>2.0 \text { AND } g-r<0.9
$$

This was used to find $g$ dropouts.

$$
g-r>2.0 \text { AND } r-i>0.3
$$

These two were used to find objects at the highest redshifts.

$$
\begin{aligned}
& r-i>1.0 \\
& i-z>1.0
\end{aligned}
$$

On the last field observed in addition to the above selection the five excluded objects with $z<20$ and $i<20$ were included. 


\subsubsection{Second Generation Targeting Algorithm}

Taking the lessons learned from the initial LBG search in July, 2001 a second generation algorithm was developed. Several key concerns were addressed, chief among them the number of stars masquerading as extended objects. In addition low redshift galaxy contaminants were also addressed. The other major development was the increasing size of the SDSS database with continuing observations over time.

\section{General Requirements}

At the time of the second generation targeting there were a number of databases to choose from. At present the SDSS is far more consolidated and usable. The Stage 1 and Stage 2 databases, which no longer exist, were used. The stage databases were advertised as having the most up to date photometry for all runs. The Chunk database contained the photometry used for drilling the spectroscopic plates. Over time the SDSS photometric pipeline is incrementally improved and new photometry is measured from the imaging for all objects. In many cases the differences in photometry are academic especially between the most recent versions of the pipeline. There were some substantial changes between the EDR and later versions. For galaxy clustering power spectrum work and other projects where completeness is a key issue it is important to have the photometry used for plate drilling as well as the most up to date photometry. However this project pushes the photometry to its limits so at every point the newest photometry is used. The Stage 1 and Stage 2 databases were identical in design. There was a limitation to the number of objects that could fit in

each database so the data was split across two databases. To conduct a full search on the 
SDSS both Stage 1 and 2 needed to be queried.

Searches were conducted on the PhotoPrimary and Galaxy classes. The PhotoPrimary class contains the main observation of each object, after removing duplicate observations from regions of overlap. The Galaxy class is the same as the PhotoPrimary class but contains only those objects which are classified as galaxies. In practice there were duplicate observations of at least some objects across different runs. Also, it was not clear if this removal of overlapping regions applies across the Stage 1 and Stage 2 databases. There is also a PhotoSecondary class where the duplicate observations are stored. Data classes were replaced by similarly defined data tables in later incarnations of the database.

Accessing the database requires the construction of a query written in the SQL language. Beyond the selection of the target class there were two general requirements in the query. The first was that the parameter objc_type $=3$, indicating the object in question is a galaxy. The classification is made by comparing the PSF magnitude to the model magnitude. This was not a requirement in the earlier search. Known lensed LBGs like cB58 and the $8 \mathrm{O}^{\prime}$ Clock Arc are extended in nature and are therefore classified as galaxies in the SDSS. It is also possible in some lensing geometries for the lensed image of a galaxy to be compact enough to be mistaken for a point source by the SDSS. In light of this fact it is not unreasonable to run the same query on objects classified by the SDSS as stars or "unknown." However given the problems with stellar contaminants with the earlier algorithm it was considered prudent to limit the search to the galaxy class.

A magnitude requirement of $17.0<i<20.5$ using model magnitudes was enforced. Model magnitudes are the recommended magnitude type for faint extended objects. This 
is a departure from the earlier algorithm which used fixed aperture fiber magnitudes. At the begining of the project magnitude limits were made in the $i$ band with the ambitious goal of finding $r$-band dropouts in mind. In general when using SDSS data cuts are made in the $r$ band because it is the band with the highest signal to noise. At this point the use of the $i$-band to set limits is only to maintain consistency with earlier phases of the project. The lower limit is set because essentially all galaxies with magnitudes brighter than 17 are automatically slated for spectroscopic followup in the SDSS Main Galaxy Survey.

On the other end the story is much more involved. Magnitudes in the SDSS are measured using luptitudes as discussed in $\$ 4.2 .1$. In the first version of the LBG selection algorithm a decision was made to limit targets to objects that were $10 \sigma$ detections. These limits can easily be calculated by setting $f / f_{0}=10 b$ using equation 4.1 and Table 4.1. The resulting limits are $u=22.12, g=22.60, r=22.29, i=21.85$, and $z=20.32$. In practice these limits seem optimistic. While these limits may be accurate for point sources they do not hold up for extended objects. To reduce the number of objects with spurious photometry the magnitude limit was set to $i=20.5$. Another aspect of the problem is that the $i-z$ color for LBGs with redshifts between 2 and 4.5 is very nearly 0 . As a result our $i$ band limit is strongly tied into our $z$ band limit.

\section{Color Space}

At the begining of the project targets were selected solely by their location in color space. At first the selection was a simple color cut that contained the LBG track above a redshift around 2 with no upper limit on the distance from the stellar and galactic loci. This approach yielded a staggering number of candidates. Because the stellar locus partially 
overlaps the LBG track and was the primary source of contaminants an attempt was made to remove stars using an additional color cut. The stelllar locus was characterized using' polygons in the three planes of color space. These polygons as well as the stellar locus in all three color planes are shown in Figure 4.8. These polygons partially overlapped the region of interest for LBGs. If an object was found to be in all three polygons it was rejected as a star. By requiring an object to be in all three polygons to reject it we were able to reduce the number of contaminants while preventing objects of interest from being eliminated.

As aforementioned the algorithm was updated to investigate only those objects classified as galaxies. A large reason for this is that quasar surveys already exhaustively search through point source objects so the odds of finding something new are much lower than the comparitively less studied extended sources. This along with the development of other methods for rejecting point sources eliminated the need for a color cut based on the stellar locus, despite the fact that a lot of time was spent on characterizing the stellar locus in color space.

By later introducing photometric redshifts to our selection we found that only LBGs with redshifts between 2.75 and 3.75 can be reliably separated from low redshift galaxies. That discovery will be discussed later in the section on photometric redshifts. To arrive at this conclusion, one must first be able to model a sample population of LBGs. To accomplish this, the first step is to be able to determine the magnitude errors for each bandpass. As mentioned before the theoretical values of the error are optinistic. To determine the errors empirically we ran a querry selecting all galaxy objects within our magnitude limits in a small portion of the sky to control the number of objects returned. 
The log of the error in each band follows a linear trend with respect to magnitude up to a cutoff magnitude. At this point the error turns over and starts to decrease with increasing magnitude. This cutoff point is slightly brighter than the $10 \sigma$ detection magnitude in each band. These distributions were fit to a line using a ruler, ignoring the points beyond the cutoff magnitude. Linear least squares fitting was attempted first, but it did not yield a satisfactory fit as it was too heavily affected by the comparitively few points with large scatter. The scatter is asymmetric, as there is an lower limit on the error at each magnitude, but no upper limit on how poorly model fitting represents a particular object. The parameterization of the model magnitudes used in given here:

$$
\begin{aligned}
& \log \sigma_{u}=0.379 u-8.91 \\
& \log \sigma_{g}=0.386 g-9.47 \\
& \log \sigma_{r}=0.382 r-9.24 \\
& \log \sigma_{i}=0.392 i-9.24 \\
& \log \sigma_{z}=0.395 z-8.63
\end{aligned}
$$

It is interesting to note that the slopes of these lines differ little from each other. The primary difference is the constant term which controls the relative strength of the error.

With the increasing size of the SDSS database it became less and less feasible to run weakly constrained searches as the number of objects returned presented several problems. One of these is disk space. Keeping the full array of photometric information takes up a lot of space. Reducing the number of parameters returned for each object can cut down on the amount of disk space considerably. However as making repeated database queries to retrieve 
individual parameters initially excluded is tedious there is a definite motive to keep as much information as possible. Another issue is the random access memory needed to simply open large files. As some of the steps in the algorithm can be computationally expensive there a strong benefit to get rid of objects at the database stage before spending CPU time on them if it is at all prudent. Lastly, at the time the connection to the database was flakey at times. Queries that took a long time ran the risk of being interrupted midstream. It was often hit or miss to successfully run a query that returned a large number of objects.

To reduce the number of objects returned for storage, computational and connectivity reasons steps were taken to better characterize potential targets. Using the theoretical LBG track plotted in Figures 4.4, 4.5, and 4.6 in conjunction with the photometric redshift program described below it was determined that only LBGs in the redshift range $2.75<z<3.75$ could be discerned from low redshift galaxies. Again using the theoretical track model LBGs were generated by adding errors to LBGs at each redshift from 2.75 to 3.75 using a range of apparent magnitudes and the relationships from equations $4.17,4.18$, $4.19,4.20$, and 4.21 .

Regions were then defined in color space to enclose the model $2.75<z<3.75$ LBGs. Model LBGs in this redshift range satisfy all of the following criteria:

$$
\begin{aligned}
& g-r \leq 1.25 \\
& g-r \geq 0.10 \\
& r-i \geq-0.20 \\
& i-z \geq-0.6
\end{aligned}
$$




$$
\begin{aligned}
& i-z \leq-3.5(r-i)+0.7 \\
& i-z \geq-4.0(r-i)+0.6 \\
& i-z \leq 4.667(r-i)+0.7
\end{aligned}
$$

These color space requirements, combined with the $i$-band magnitude limits and galaxy classification requirement were then combined to make this SQL database query

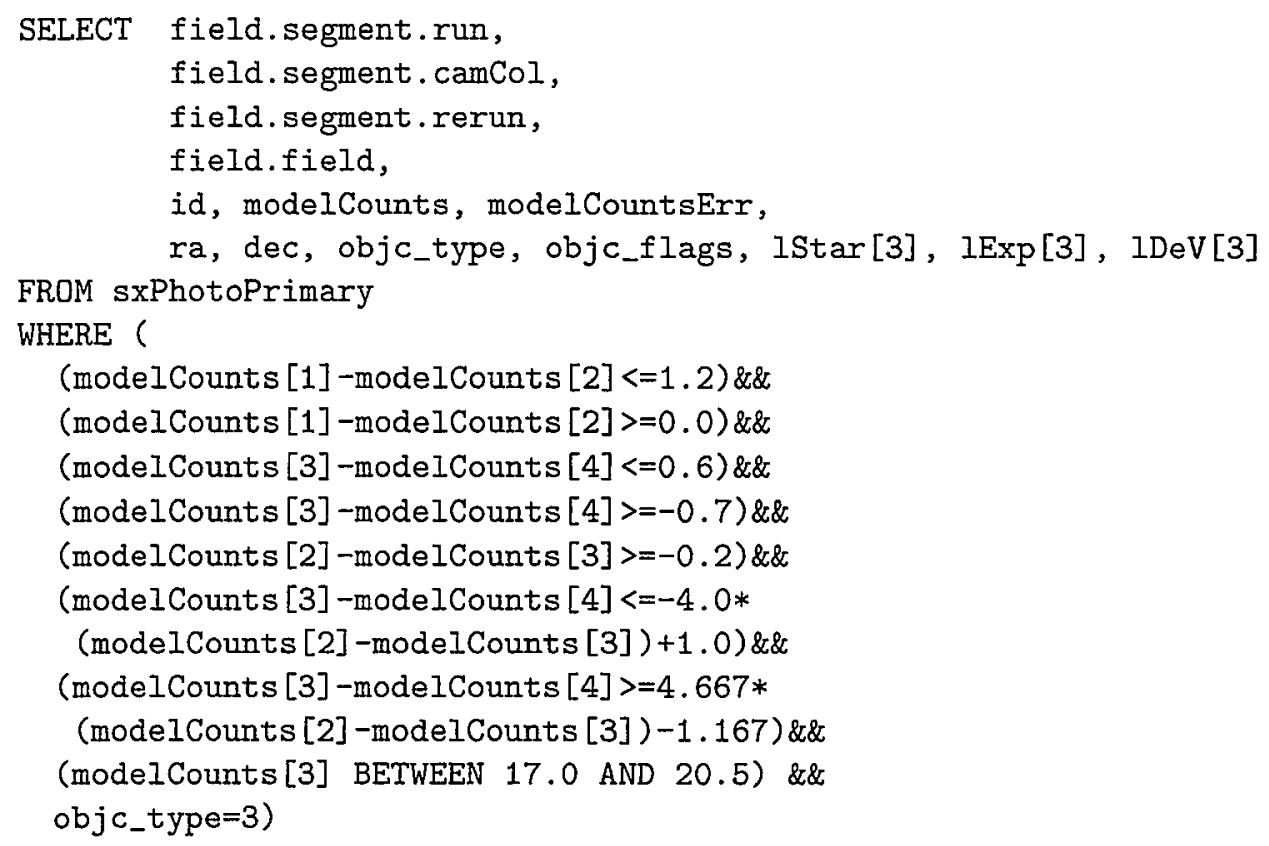

\section{Flag Tests}

The flag filter was not changed from the original first generation algorithm. The same six flags were used as was the requirement that the sum of the photometry errors fall between 0 and 8 although the errors were changed from fiber magnitude errors to model magnitude errors to match the change from fiber magnitudes to model magnitudes.

On average the combination of flag and error checking done will reject $50 \%$ of 
the objects initially returned by the database query. For this reason, and because it is computationally fast, it is the optimal choice for the first step after querrying the database.

\section{Advanced Star Separation}

After the first $2 \mathrm{dF}$ observing run on this project it became painfully clear that the SDSS star-galaxy separation was not sufficient for this project. Many of the objects observed were A stars. The SDSS star-galaxy separation is based on the difference between the PSF magnitude and the best fit of the model magnitudes. If $m_{p s f}-m_{\text {model }}>0.145$ then the object is classified a galaxy in that band, otherwise it is a star. This is advertised to work at the $95 \%$ confidence level to $r=21$ (Stoughton, et al. 2002).

Given the large number of objects that we are scanning and the proximity of A stars to LBGs in color space this is not sufficient. To further prevent contamination by stars the likelihood of fit to model parameters in the SDSS photometric data are used. These are the star_L, deV_L and exp_L parameters, which are likelihoods that an object is well represented by a PSF, deVaucoleurs and exponetial profile respectively. The values at the time were probabilities ranging between 0 and 1 . They have since switched to log likelihoods. These likelihoods can be used together to get a fractional likelihood:

$$
P(P S F)=\frac{\operatorname{star} L}{\operatorname{star} \_+\operatorname{deV}_{-} L+\exp L}
$$

For searches using model magnitudes it was required that $P(P S F)<0.015$.

Because stars remained our largest contaminant yet another step was taken to reduce their number. Using the Pickles database of empirical stellar templates (Pickles 1998), we compared the fluxes in all bandpasses for each object to the spectral energy 
distributions (SEDs) of 108 stars ranging from spectral type 05 to M10 and luminosity classes I through V. By convolving the spectrum of each of the template stars with the SDSS filter response curves we were able to find the fluxes in each band of the templates. The $\chi^{2}$ of the difference between the template fluxes and oberserved fluxes were calculated by the following:

$$
\chi^{2}=\sum_{i}\left[\frac{F_{o, i}-b F_{t, i}}{\sigma_{i}}\right]^{2}
$$

This can be converted to a formula for magnitudes via $m=-2.5 \log f+c=-1.086 \ln f+c$

$$
\chi^{2}=1.086^{2} \sum_{i}\left[\frac{1-b 10^{\left(m_{o, i}-m_{t, i}\right) / 2.5}}{\sigma_{m_{i}}}\right]^{2}
$$

where $m_{t, i}$ and $m_{o, i}$ are the star template and object magnitudes in the $i$ th bandpass and $b$ is coefficient for the template flux that minimizes the value of $\chi^{2}$. It can be derived analyticaly using

$$
b=\frac{\sum_{i}\left(\frac{10^{\left(m_{o, i}-m_{l, i}\right) / 2.5}}{\sigma_{m_{i}}^{2}}\right)}{\sum_{i}\left(\frac{10^{2\left(m_{o, i}-m_{t, i}\right) / 2.5}}{\sigma_{m_{i}}^{2}}\right)}
$$

From this $\chi^{2}$ we calculted the likelihood that the object matched the stellar template. If this likelihood exceeded 0.015 for any of the 108 templates an object was rejected.

Since implementing the use of three independent star rejection methods no single stars have been mistakenly observed. This is not the end of the issue as objects separated by less than 2 arcseconds can be misidentified as single objects by the deblender. Binary star systems can have combined fluxes that do not resemble those of a single star and mimmick those of galaxies. One way to prevent this from happening is to inspect the images, which is discussed later. However, observations at Subaru were able to clearly resolve a binary system where no hint was given by the SDSS image. To combat this problem the flux comparison 
between an observed object and single stellar templates was extended to comparisons with the sum of two templates. The earlier $\chi^{2}$ expression is extended to

$$
\chi^{2}=\sum_{i}\left[\frac{F_{o, i}-a A_{i}-b B_{i}}{\sigma_{i}}\right]^{2}
$$

where $F_{o, i}, A_{i}$ and $B_{i}$ are the fluxes of the observed object, first template and second template in the $i$ th bandpasss, and $a$ and $b$ are the flux weighting coefficients that minimize the chi square. This can be converted to an expression involving magnitudes.

$$
\chi^{2}=1.086^{2} \sum_{i}\left[\frac{1-a 10^{\left(m_{o, i}-m_{\Lambda, i}\right) / 2.5}-b 10^{\left(m_{\circ, i}-m_{B, i}\right) / 2.5}}{\sigma_{m_{i}}}\right]^{2}
$$

Once again the optimal values for the flux weighting coefficients can be determined analytically, albeit not as easily:

$$
a=\frac{A B^{2}-B \sum_{i}\left(\frac{10^{\left(2 m_{0, i}-m_{A, i}-m_{B, i}\right) / 2.5}}{\sigma_{m_{i}}^{2}}\right)}{A^{2} B^{2}-\left(\sum_{i} \frac{10^{\left(2 m_{0, i}-m_{A, i}-m_{B, i}\right) / 2.5}}{\sigma_{m_{i}}^{2}}\right)^{2}}
$$

and

$$
b=\frac{B A^{2}-A \sum_{i}\left(\frac{10^{\left(2 m_{o, i}-m_{A, i}-m_{B, i}\right) / 2.5}}{\sigma_{m_{i}}^{2}}\right)}{A^{2} B^{2}-\left(\sum_{i} \frac{10^{\left(2 m_{o, i}-m_{A, i}-m_{B, i}\right) / 2.5}}{\sigma_{m_{i}}^{2}}\right)^{2}}
$$

where

$$
A=\sum_{i}\left(\frac{10^{\left(m_{o, i}-m_{A, i}\right) / 2.5}}{\sigma_{m_{i}}^{2}}\right)
$$

and

$$
B=\sum_{i}\left(\frac{10^{\left(m_{o, i}-m_{B, i}\right) / 2.5}}{\sigma_{m_{i}}^{2}}\right)
$$

To use this as criteria would eliminate all targets, so it is only used after the fact. This is not as bad as it sounds as galaxy spectra are the sum of stellar spectra. It is not surprising that they may be aproximated by the sum of two stars, especially considering 
that there are 11,556 different combinations of 108 templates. To our advantage, the 11,556 combinations can be narrowed down using two facts. The first is that it is far more likely for two stars within two arcseconds of each other to be in a binary association, and thus at the same distance, than it is for a chance alignment. The second is that the relative luminosities of the spectral types and luminosity classes are well established. Using the best fit flux weighting coefficients and stellar types for the best fitting pairs it can be determined whether a pair can plausibly be a binary system.

\section{Photometric Redshifts}

A feature new to the second generation algorithm is the use of photometric redshifts. Photometric redshifts are estimates of an object's redshift using wide band photometry. To find the true redshift of an object spectroscopy is still required. Photometric redshifts work using the same SED fitting procedure outlined in the section on star rejection. However, in this case redshift, extinction and reddening must also be applied to the templates. The success of photometric redshifts is strongly related to the number of bandpasses used and the overall range in wavelength covered. Ideally infrared photometry in the $J, H$ and $K$ bands could be used to improve the performance of the photometric redshifts. However because of the magnitude limits imposed objects are too faint to be found in the 2MASS catalog.

Photometrie redshifts are calculated using the publicly availible software Hyperz v1.1 (Bolzonella, Miralles, \& Pelló 2000). Seven built in templates to measure the redshift, covering the elliptical, $\mathrm{S} 0, \mathrm{Sa}, \mathrm{Sb}, \mathrm{Sc}, \mathrm{Sd}$, and irregular galaxy types. These templates take into account changing star formation rate over the age of the galaxy. The $\mathrm{E}, \mathrm{S}$, $\mathrm{Sa}, \mathrm{Sb}$, 
Sc, and Sd templates have exponentially decaying star formation rates with timescales of $1,2,3,4,15$, and $30 \mathrm{Gyr}$, while the Im template has a constant rate of star formation.

In using Hyperz there are a large number of input parameters that are set in a main parameter file. The choice of templates is made in a separate parameter file. The FILTERS_FILE parameter gives the name of the file which contains information on the filter system used, and how to treat non-detections as well as the magnitude system being used. For objects fainter than the limiting magnitude in a filter the option for which the observed flux is set to zero with an error equal to the flux associated with the limiting magnitude was selected.

The MAG_TYPE parameter gives a choice of Vega or AB magnitudes. As elucidated in section 4.2.1 the SDSS uses its own luptitude system. While the difference between luptitudes and the $A B$ system disappears for bright objects for faint objects and non-detections it can be significant. Hyperz includes values for the SDSS limiting magnitudes $, 22.7,23.9,23.7,23.2$ and 21.3 for the $u, g, r, i$ and $z$ filters. It is unclear where these numbers came from. The limiting magnitudes correspond to $5.8,2.7,2.4,2.6$ and $3.8 \sigma$ sky noise for the $u, g, r, i$ and $z$ filters respectively. When the limiting magnitudes in the table are converted to $\mathrm{AB}$ magnitudes they become 22.73, 24.02, 23.85, 23.34 and 21.36.

There are several other less contraversial parameters whose values should be mentioned. The ERR_MAG_MIN parameter sets a lower limit on the photometric error, which is set to 0.03 . It is not unusual for the error to be less than this for objects with $g$ and $r$ magnitudes less than 20. However the systematic error is probably at least 0.03 especially considering known problems with model magnitudes at the time. The $\mathbf{Z}_{-} \mathbf{M I N}$, 
Z_MAX and Z_STEP parameters control the range of redshift values tested and the increment between steps. Redshifts from 0.00 to 6.00 were covered in steps of 0.05 . The reddening law is controlled with the REDDENING_LAW. The starburst galaxy reddening law from Calzetti, et al. (2000) was used. The reddening law uses the the AV_MIN, AV_MAX, AV_STEP to control the range of values of absorption in the $V$ band and the step taken. The range was set from 0.00 to 2.50 magnitudes in steps of 0.25 . The number of reddening values checked by the program is limited, which explains the coarse resolution used. By turning on the AGE_CHECK parameter Hyperz checks to make sure that the age of the template it is using is less than the age of the universe. Since real data is being used confusion can be avoided by eliminating solutions that are not currently possible. The H0, OMEGA_M, and OMEGA_V parameters are set to the conventional values of $H_{0}=70, \Omega_{M}=0.3$ and $\Omega_{V}=0.7$.

Before using Hyperz the data is properly formated for Hyperz to read. In addition all magnitudes less than the respective limiting magnitude discussed above are replaced with 99.00 to signal that Hyperz should consider it a nondetection. The next step is to run Hyperz. Hyperz has two main outputs. The first is the .z_phot file which contaings among other things the photometric redshift and associated $\chi^{2}$ and probability of the primary solution for each object. The second output is the .log_file which gives the best fit redshift, $\chi^{2}$, probability, template type, age and $V$ band reddening for each redshift increment for vach object.

After passing the data through Hyperz steps are taken to interpret the results. Many objects have high likelihoods at high redshifts. However many of these simultaneously 
have large likelihood peaks at low redshifts. From the SED fitting perspective as vicwed by Hyperz an object may correctly fit galaxy templates in the low and high redshift universe equally well. The top panel of Figure 4.9 gives an example of the Hyperz results for an object with strong peaks at both high and low redshifts.

It is an indisputable observational fact that low redshift galaxies overwhelmingly outnumber exceptionally luminous high redshift objects. In truth while Hyperz may find it equally likely that the distance to an object is small or cosmological it is clear that any such object is almost certainly a low redshift contaminant.

To avoid this trap it is required that the maximum probability of a match for any of the seven templates for $z \leq 1$ be less than 0.01 . We also require that the probability of a match of at least one template be larger than 0.50 for $2.75 \leq z \leq 3.75$. As previously mentioned, by running LBG models through Hyperz it saw determined that LBGs outside this redshift range cannot be distinguished from low redshift galaxies.

\subsubsection{Current LBG Selection}

In 2006 the search for strongly lensed LBGs was revisited. While the second generation selection was a significant departure from the first generation selection the present selection is primarily upgrades and improvements on the second generation algorithm. There were several motivations for returning to the LBG search. Chief among them is that over the intervening years the SDSS increased significantly in size allowing now arcas of the sky to be searched for the very first time. In addition the SDSS observed cB58. For the first time there were observations of a real LBG to test the algorithm against. The improved organization of the SDSS database for the first time made it feasible to access the imaging 
for all objects. This opened new doors for determining the merit of a candidate.

The current LBG selection is specifically designed to be as harsh as possible yet still allow cB58 to pass through.

\section{SDSS Query, Flag Filer and Star Separation}

Given the improvements in the SDSS database, particularly the CAS (Catalog Archive Server) the entirety of data releases can be accessed from a single database. DR5 (Adelman-McCarthy, et al. 2007) was used for the latest search. DR5 contains photometry for $8000 \mathrm{deg}^{2}$ which includes 215 million unique objects.

The database query has several differences from the previous algorithm. The same magnitude cut of $i<20.5$ in model magnitudes is applied, as is the requirement that the objects be classified as extended sources by the SDSS. However the color cuts from equations 4.22 through 4.28 are eliminated. The main reason for this is that $\mathrm{cB} 58$ does not meet these requirements and is rejected by the second generation algorithm. Clearly equations 4.22 through 4.28 needed to be fixed. The peripheral goal of this project in addition to finding LBGs is to keep an eye out for new, as yet undiscovered classes of objects. Imposing the tight color space restriction reduced the opportunities for serendipitous discoveries. In addition the project has moved from straight color cuts to $\chi^{2}$ fitting with spectral templates. When the photometry is precise color cuts are a computationally economical and effective way to sort, classify and define individual objects and populations. Howevor when the photomotry becomes imprecise some color indices become highly uncertain and have effectively arbitrary values. This is a particular problem in the SDSS where each bandpass has a different sensitivity, and the $u$ band in particular is of a lesser quality than the other bands. Color 
cuts use only two photometry bands at once which can be problematic when one band is poorly detected and noisy. The $\chi^{2}$ approaches, while more computationally expensive, use all five bands at once to holistically identify the nature of an object and are thus more able to overcome poor data quality in a single band. In addition $\chi^{2}$ techniques express results as a probability which can be interpreted with shades of gray rather than a straight yes or no determination from a color cut.

The query was also modified such that objects with $r_{\text {petro }}<17.77$ are rejected. These objects fall in the SDSS Main Galaxy Survey and are automatically targeted for follow up spectroscopy. In order to reduce the number of stellar contaminants it is required that $i_{\text {psf }}-i_{\text {model }}>0.3$. The SDSS photometric pipeline uses 0.145 as the cutoff value but from experience with the first generation algorithm many stars elude this test. Finally to reduce the number of objects with spurious photometry it was required that the model magnitude error be less than 0.1 in the $i$-band and less than 0.15 in the $z$-band. While candidate galaxies may be dropouts in the $u$ and $g$, and perhaps even $r$-band all candidates should be well detected in the $i$ and $z$ bands. As a result this criteria can significantly reduce the number of objects returned without eliminating any quality candidates.

The flag filter is also updated. A key change in the flag filter is that in this version the $r$-band flags are used as opposed to the combined flags. Flags are first set in each of the five photometric bands individually. If a flag is set in any one or more of the $u, g, r, i$, and $z$ bands it will be set in the combined flags. However the $z$ and $u$ bands have much lower $\mathrm{S} / \mathrm{N}$ than the other three bands. A photometry problem in one of these bands could be a result of the $\mathrm{S} / \mathrm{N}$ and not reflective of the photometry quality of the other bands. This is 
especially pertinent in this project which pushes against the limits of the survey. The latest. version of the flag filter uses only the $r$-band flags as the $r$-band has the highest $\mathrm{S} / \mathrm{N}$.

As in the previous versions objects with the flags EDGE, CR, SATUR are rejected. Previously objects with the BLENDED and CHILD flags set were rejected. However cB58 has both of these flags set. The correct treatment of the BLENDED flag is to allow not only the objects for which the flag is not set, but also those for which the flag is set provided that the NODEBLEND flag is also set. The BLENDED flag is set when multiple peaks are found in the profile of an object indicating that it is a possible blend of two or more objects. These objects are subsequently sent to the deblender which either separates an object into a parent and child or children or determines that upon closer inspection the object is not a blend. In the latter case the NODEBLEND flag is set to indicate that no further action was taken. Therefore an object with both the BLENDED and NODEBLEND flags set has not been affected by the deblender. The reason behind eliminating blended objects is the concern that the deblender may introduce errors into the photometry of objects, especially near the survey limits. There is no reason to eliminate objects which were not in the end deblended as their photometry is not affected in this manner. In fact, there are a couple of reasons to keep objects with multiple peaks in the profile in the context of a search for lensed LBGs. Multiple peaks can be a natural result of gravitational lens, particularly when the an object is being lensed by something with a complicated mass profile. Since we expect. LBGs to be lensed by foreground clusters which have potentially intricate structures multiple peaks are a real possibility. The other reason is that LBGs are young galaxies with active star formation. If this star formation takes place in localized areas as opposed to uniformly 
through the galaxy a multiple peaked profile could result.

A few additional changes to the flag filter were also made. These changes are designed to reduce the number of objects in the sample before moving to more computationally expensive $\chi^{2}$ steps. Objects with the BRIGHT flag set are rejected. This flag is set for objects which are detected in the first pass of the photo pipeline and have $r<17.5$. Technically this should be redundant as it is already required that $r_{\text {petro }}>17.77$. However in practice this rejects a small number of objects. Objects with the NOPETRO and MANYPETRO flags set are also rejected. These are objects for which the photometric pipeline has diffculty measuring a Petrosian radius. The inability of the pipeline to determine a profile is indicative of a poor $\mathrm{S} / \mathrm{N}$ detection. The NOPETRO and MANYPETRO are two examples of the dangers of using the SDSS photometry flags blindly. The SDSS webpage points out that half of the galaxies in the Main Galaxy Sample (those with $r_{\text {petro }} \leq 17.77$ ) have NOPETRO set in the combined flags but only $\sim 2 \%$ in the $r$-band flags. This is because of low $\mathrm{S} / \mathrm{N}$ in the $u$ and $z$ bands where a Petrosian profile is not found while the galaxies are well detected in $r$. Lastly objects with the INTERP flag set are rejected. These objects have one or more pixels in the $r$-band whose values are the result of an interpolation. This can happen due to cosmic rays, deblending, saturated pixels or other reasons. The collective goal of all of the photometry flag requirements is to ensure that the $r$-band photometry is robust- the product of direct measurements and not based on interpolation. If the $r$-band flux cannot be trusted in the magnitude regions targeted in this project it is unlikely that any of the other bands can be believed. The other goal is to create a flag filter which is as strict as reasonably possible which allows the SDSS observations of cB58 to pass. The requirements 
here do not reject $\mathrm{cB58}$.

Advanced star separation is carried out in exactly the same mamner as for the second generation algorithm. These criteria were detailed in equations $4.29,4.31$, and 4.32 . One change in the star separation is in the database query. As mentioned earlier it is required that $i_{p s f}-i_{\text {model }}>0.3$. The other change is that the binary star fitting technique given in equations $4.33,4.34,4.35$, and 4.36 is not used. The binary star technique rejects nearly every object of interest so it is not particularly useful.

\section{Hyperz Upgrage}

A key upgrade for the current selection algorithm is the alteration made to Hyperz. Hyperz has the built-in ability to handle both Vega magnitudes and AB magnitudes. As previously discussed the SDSS data does not use either of these systems, but rather uses its own system of luptitudes, or $\sinh ^{-1}$ magnitudes. In the earlier use of Hyperz the differences between the luptitude and $A B$ system were ignored and approximate limiting magnitudes were used as described earlier. The Hyperz source code was modified to allow luptitudes as a third choice for the MAG_TYPE parameter. By selecting this option the SDSS magnitudes are converted to fluxes directly using equation 4.3 and the SDSS softening parameters from Table 4.1. In this scenario there are no limiting magnitudes because negative fluxes can be handled by luptitudes. By correctly handling luptitudes no photometric information is lost.

Another important modification to the Hyperz code is the addition of the model LBG spectrum from Figure 4.3 to the list of templates used for determining photometric redshifts. The LBG spectrum is similar to the Sd template in that both have young stellar 
populations with active star formation. However the LBG spectrum is even younger with stronger star formation activity. By adding the LBG template the parameter space is more fully covered. It also makes it possible to fit for LBGs directly.

Figure 4.9 gives an example of the difference between the old and new Hyperz results for $\mathrm{cB} 58$. For a well exposed object like cB58 treating the luptitudes like AB magnitudes is not a bad approximation and the difference in results is minimal. In the upgraded results the redshift resolution is reduced because the number of extinction models has been increased. The key result of Figure 4.9 is that a true LBG like cB58 can have strong peaks at both $z=0$ and $z>2$. In the second generation algorithm $\mathrm{cB} 58$ would be rejected for having a high probability of $z=0.0$. Figure 4.9 also demonstrates how difficult it is to pick LBGs out of the SDSS from photometry alone.

\section{Cross-Referencing Against Known Galaxy Clusters}

The strongly lensed galaxies which have been found all share the common characteristic that they were found serendipitously in either galaxy cluster studies, or in the case of the $8 \mathrm{O}^{\prime}$ Clock Arc, in surveys of interacting galaxies. Strongly lensed LBGs are expected to be lensed by intervening massive galaxy clusters. However, these clusters may or may not be known at present. Given the geometries of lens systems it is well within the range of possibilities that a massive cluster lensing an LBG may be at a distance which makes it appear too faint to be easily observable. For this reason this projoct was dosigned as a fully photometric search which targets LBGs regardless of whether they are located in clusters or not. A purely photometric search, if successful, could discover lensed LBGs away from known clusters which have far less chance of serendipitous discovery than those in well 
studied galaxy clusters. Furthermore finding LBGs lensed by unknown, unseen chusters could provide valuable information about the number and characteristics of galaxy clusters during an epoch of history which is not easily observable at present.

While it is clearly preferable to place as few external restrictions on the photometric search as possible the fact remains that earlier versions of the selection algorithm have so far failed to discover any LBGs. In light of this reality the decision was made to pay particular attention to regions near known galaxy clusters in hopes of improving the odds of finding something. Images of all objects returned by the SDSS query that survive the flag filter and are within an arcminute of a known galaxy cluster are inspected by eye. This is not to the exclusion of objects not near known clusters which are still looked at if their Hyperz results are encouraging.

Eight publicly available galaxy cluster catalogs were downloaded from the internet from NASA's HEASARC archive. Each of these catalogs were queried to return all clusters with declinations greater than $-20^{\circ}$ and redshifts $z>0.15$. The cut with declination is due to the fact that the footprint of the SDSS does not extend that far south as the telescope is located in the Northern Hemisphere. No SDSS objects will overlap with galaxy clusters at such extreme southernly declinations. The redshift cut is used because clusters in the local universe will not have favorable geometries to lens LBGs in the redshift regions of interest. The redshift cut rejects a significant number of clusters that can be ignored to save time without compromising the results.

The locations of clusters in equatorial coordinates from the eight catalogs are shown in Figure 4.10. There is considerable overlap between several of the groups-many 
objects appear in two or more catalogs. The eight galaxy cluster catalogs are briefly described here.

The Abell Catalog of Rich Clusters of Galaxies was created from the analysis of photographic plates (Abell, Corwin, \& Olowin 1989). The plates come from the Palomar Sky Survey in the northern sky and the UK Schmidt Telescope in the south. An important caveat is that the catalog is based only on the apparent densities of galaxies in visual wavelengths. Clusters are included if they contain at least 30 galaxies within two magnitudes of the third brightest cluster member and have redshifts less than $z=0.2$. The Abell catalog contains 4073 rich clusters of which 132 meet our criteria. Of these objects 52 have redshifts of $z>0.2$, with a maximum of $z=0.373$, presumably because certain Abell clusters were later found to be at larger distances with more accurate measurements.

The Faint Sky Variability Survey (FSVS) Catalog of Galaxy Clusters and Rich Groups is, like the Abell catalog, an optically selected galaxy cluster catalog (Söchting, et al. 2006). The catalog was created from $B, V$, and $I$ imaging from the $2.5 \mathrm{~m}$ Isaac Newton Telescope at La Palma of $\sim 23 \mathrm{deg}^{2}$ out of the galactic plane. The imaging is fairly deep; it reaches 25th magnitude in the $V$ band. As can be seen in Figure 4.10 the FSVS catalog finds the highest density of clusters, but only in a handful of fields spread across the northern sky. Cluster identification follows the same basic idea as used by the Abell catalog. Over-densities of galaxies are identified. However the method used is automated and far more advanced. On the downside the redshifts are not spectroscopically determined but rather estimated from the cluster colors. In spite of the small area covered the FSVS cluster catalog contains 598 galaxies which is comparable to the all sky X-ray surveys described 
below. Of those 488 meet the criteria set out above. The mean redshift estimate for these galaxies is $z=0.39$ and range as high as $z=0.94$. The FSVS cluster catalog is unicue among the catalogs used here in that it is a very deep optical survey. In spite of its limited sky coverage it compliments the other catalogs nicely.

The Einstein Observatory Clusters of Galaxies Catalog contains galaxies observed with the Imaging Proportional Counter on the Einstein Observatory with redshifts $z<0.2$ (Jones \& Forman 1999). The Einstein Observatory was the first X-ray telescope in space with full imaging capabilities. It was in operation from 1978 to 1981. Fluxes are measured in the 0.5 to $4.5 \mathrm{keV}$ band. Of the 368 clusters in the Einstein catalog 54 fit the criteria described above. Of these objects 8 were later determined to have redshifts in excess of $z=0.2$ with a maximum of $z=0.26$.

The ROSAT All-Sky Survey (RASS) Brightest Cluster Sample (Ebeling, et al. 1998) and Low-Flux Extension (Ebeling, et al. 2000) is a sample of galaxy clusters in the northern hemisphere with X-ray fluxes in excess of $2.8 \times 10^{-12} \mathrm{erg} \mathrm{s}^{-1} \mathrm{~cm}^{-2}$ in the 0.1 to 2.4 keV energy band. The data come from ROSAT (short for Röntgensatellit) a German built space based X-ray observatory which launched in 1990 and operated until 1999. Around $70 \%$ of the clusters in the Brightest Cluster Sample are in the Abell catalog and $20 \%$ are detected purely in the X-rays. The completeness is measured to be $90 \%$ for the Brightest Cluster Sample and 75\% for the Low-Flux Extension. Between the two parts there are 300 galaxics in the semple, of which 99 meet the above criteria.

REFLEX is the ROSAT-ESO Flux-Limited X-Ray Galaxy Cluster Survey Catalog (Böhringer, et al. 2004). Galaxy cluster candidates were selected by their X-ray emission in 
the RASS. Targets were the spectroscopically identified as part of an ESO program. REFLEX is estimated to be $90 \%$ complete for clusters with fluxes above $3 \times 10^{-12} \mathrm{erg} \mathrm{s}^{-1} \mathrm{~cm}^{-2}$ in the 0.1 to $2.4 \mathrm{keV}$ X-ray bandpass. The REFLEX survey covers an area of 4.24 steradians in the southern sky. As a result only 44 of the 447 clusters in REFLEX survive the declination and redshift requirement.

Böhringer, et al. (2000) describe the Northern ROSAT All-Sky (NORAS) Galaxy Cluster Survey. The NORAS cluster catalog is an X-ray selected sample of galaxy clusters designed for cosmological studies. Like the REFLEX survey the data for the NORAS catalog came from RASS. The NORAS cluster catalog is complementary to the REFLEX catalog as it is composed of objects from the northern sky. The NORAS catalog differs from REFLEX in that it is selected purely from X-ray data. The REFLEX survey relies on the the correlation of X-ray sources with known galaxy over-densities measured in optical bands. The NORAS cluster survey targets extended X-ray sources and conducts optical follow-up. As such the NORAS cluster survey finds more distant galaxy clusters and reveals many clusters that were previously known from photographic surveys in the optical. Of the 495 objects in the NORAS catalog 174 meet the search criteria. The redshifts of these objects have a mean of $z=0.24$ but reach as high as $z=2.49$. The higher redshifts of these clusters compared to the other cluster catalogs make the NORAS galaxy clusters particularly good candidates as they are in the redshift range of clusters known to strongly lens LBGs.

The ROSAT PSPC Catalog of Cluster's of Galaxies is another X-ray galaxy cluster catalog created using ROSAT data (Vikhlinin, et al. 1998). The PSPCs are Position Sensitive Proportional Counters which are X-ray sensitive imagers with $\sim 25$ arcsecond spatial 
resolution. ROSAT had two PSPCs the second of which was redundant. The PSPCs were also used for the ROSAT All Sky Survey on which the REFLEX and NORAS catalogs are based. The ROSAT PSPC survey differs from the previous ROSAT catalogs in that it covers far less area on the sky, 158 square degrees, but goes considerably deeper. The range of X-ray fluxes of the ROSAT PSPC clusters is $1.6 \times 10^{-14}$ to $8 \times 10^{-12} \mathrm{erg} \mathrm{s}^{-1} \mathrm{~cm}^{-2}$ in the 0.5 to $2 \mathrm{keV}$ energy band. By consequence of the small overlap in flux range with the REFLEX and NORAS surveys there are a handfull of objects which appear in both the ROSAT PSPC catalog and either the REFLEX or NORAS catalogs. Candidate galaxy clusters are targeted by identifying objects with extended X-ray flux and are confirmed in the optical. Of the 203 galaxy clusters in the ROSAT PSPC catalog 127 survive the restrictions for this project. The fainter fluxes probed in this survey make it possible to find clusters at larger distances. The mean redshift of the surviving clusters is $z=0.294$. This range of redshifts is also in good agreement with the redshifts of clusters lensing the known strongly lensed LBGs.

The BAX (Base de Données Amas de Galaxies X) X-Ray Galaxy Clusters and Groups Catalog is a searchable multi-wavelength database of galaxy clusters and groups containing equatorial coordinates, names and identifiers, redhift, X-ray luminosities, temperatures and links to outside information (Sadat, et al. 2004). The BAX database provides no new observations but is a collection of published results on X-ray clusters. It is designed to facilitate the study of clusters and groups by putting information spread out over many unrelated published works into one searchable location. The aforementioned query returus 534 clusters and groups from BAX. In Figure 4.10 BAX appears to contain all ROSAT 
PSPC galaxies, although BAX contains many objects not included in ROSAT PSPC.

These eight galaxy cluster samples complement each other well. The Abell and FSVS catalogs are optically selected, while the other six are selected either partially or entirely on space based X-ray observations. As Figure 4.10 shows there is considerable overlap between many of the samples. However each sample contains clusters not found in the others, with the exception that all ROSAT PSPC galaxies appear to be in the BAX sample. Combined they are far from a complete sample but the goal is not to make statistically correct statements about populations of galaxy clusters. The goal is to create a list of potential lenses for LBGs. The combined sample provides a large number of galaxy clusters in the northern sky and outside of the Galactic plane with a diverse range of physical properties. As such the combined sample is more than satisfactory.

With a list of galaxy clusters in hand the next task is to identify LBG targets which fall along the line of sight to those clusters. Based on images of the known strongly lensed LBGs they tend to be relatively near the cores of the lensing clusters. For this reason the search radius for the known clusters is set somewhat arbitrarily to 1 arcminute. To measure this correctly one needs to employ spherical trigonometry. The angle $\gamma$ between two objects on the sky is given by

$$
\gamma=\arctan \left(\frac{\sin \delta_{1} \sin \delta_{2}+\cos \delta_{1} \cos \delta_{2} \cos \Delta \alpha}{\sqrt{\left(\cos \delta_{2} \sin \Delta \alpha\right)^{2}+\left(\cos \delta_{1} \sin \delta_{2}-\sin \delta_{1} \cos \delta_{2} \cos \Delta \alpha\right)^{2}}}\right)
$$

where $\alpha_{1}$ and $\delta_{1}$ are the right ascension and declination of the first object, $\alpha_{2}$ and $\delta_{2}$ are the RA and dec of the second object and $\Delta \alpha=\alpha_{1}-\alpha_{2}$. Equation 4.39 is an approximation which is considerably simpler than the full version. The value of $\gamma$ is at most $\pi / 2$ radians due to the inverse tangent; objects on opposite sides of the sky are mistakenly determined to 
be closer than they really are. Because the SDSS galaxies are all north of $-20^{\circ}$ declination and there are two large gaps due to the Galactic plane this is only an issue for a couple of clusters which can be weeded out manually.

As described earlier images of all objects which survive the database query, flag filter and advanced star separation and fall within 1 arcminute of the the core of a known galaxy cluster with $z \geq 0.15$ are inspected by eye. When inspecting the images there are two main characteristics to look for. One is the color. As described earlier LBGs have colors which are distinct from nearby galaxies. In Figure 4.11 SDSS color composite images of known strongly lensed LBGs are shown. The images of cB58 and the 8 O'Clock Arc have a distinct bluish purple appearance which stands in stark contrast to the orange galaxies of the lensing cluster. The other characteristic is the shape. Gravitational lensing often warps the shape of the image of the galaxy. Lensing often leaves the image of a galaxy elongated or, more dramatically, in an arced banana shape. The only other cause for such severe warping is galaxy interactions which are also rare and warrant a closer look. When the bend in a galaxy is concentric with the cluster core it is almost certainly the result of lensing. The image of the $8 \mathrm{O}^{\prime}$ Clock is a textbook example of gravitational lensing and a clear sign that the object should be followed up spectroscopically.

There is another significant benefit to checking targets against the known positions of galaxy clusters. As seen above in Figure 4.9 the photometric redshift results from Hyperz often have a peak at low redshift as well as one at higher redshifts. This is true even for real LBGs which makes the task of finding them photometrically all the more challenging. When looking at the Hyperz results for targets along the line of sight to clusters there is an 
extra piece of information which can be used. If the low redshift peak matches the known galaxy cluster redshift then the object in question is almost certainly a cluster member itself and can be ignored. However if the low redshift peak differs significantly from the known cluster redshift the object is of increased interest.

Figure 4.12 shows the photometric redshift results for two galaxies selected by cross-referencing with the positions of known galaxy clusters. The figure shows the photometric redshift information calculated by the version of Hyperz modified to handle luptitudes. The objects plotted demonstrate how the photometric information can be used to help make an informed judgement on target selection.

The top panel shows the photometric redshift results for the object SDSS J1547+2056. According to Hyperz it has a $4 \%$ chance of being at $z=0.05$ and $2.5 \%$ chance of being at $=2.23$. On top of that its colors are not consistent with being other redshifts. This is qualitatively similar to case of cB58, as shown in Figure 4.9, although the probability peaks are not nearly as strong. The fact that the probabilities are so low across the board suggests this object has interesting colors and a non-standard spectrum. This is especially true considering that the object is well detected with an apparent magnitude of $r=18.68$. This suggests that the strange photometric results are not due to random fluctuations in poor photometry. The cluster that SDSS J1547+2056 falls along the line of sight of has a known redshift of $z=0.23$. According to the photometric redshift results the probability that the object is at the cluster redshift is close to zero. In addition the image of SDSS J1547+2056 shows that it is near the cluster core and has colors which distinguish it from the cluster members. For these reasons SDSS J1547+2056 was observed spectroscop- 
ically. The spectrum shows that the object has a very strong Balmer break and a redshift $z=0.264$.

The middle panel of Figure 4.12 shows the photometric redshift results for another object of interest, SDSS J1640+4643. This object has a strong peak at $z=0.82$ with a probability of $87 \%$. The probabilities of being at $z=0$ and the cluster redshift, also $z=0.23$, are both $0 \%$. The bottom panel of Figure 4.12 shows the redshift results for another object which also survives the criteria of the current algorithm described above. It has a small peak of $5 \%$ at $z=3.12$ and a strong peak of $78 \%$ at $z=0.22$. Because the known cluster redshfit is $z=0.23$ this object is clearly most likely a cluster member and can be thrown out. However it is shown as an example to show why SDSS J1640+4643 stands out as an object of interest. In addition to the photometric redshift information the image of SDSS J1650+4643 is particularly intriguing. It is located about 20 " from a large elliptical galaxy at the cluster core. In addition it has an elongated shape which is stretched perpendicular to the line connecting the object and the cluster core, the direction that would be expected if caused by gravitational lensing. Spectroscopic follow up of SDSS $\mathrm{J} 1640+4643$ shows reveals that it is a galaxy with weak emission lines at a redshift of $z=0.862$. In this case the photometric redshift returned by Hyperz was correct.

\section{Color Cuts}

Sometime after the current selection algorithm was put into use the discovery of the 8 O'Clock Arc was announced. With this discovery there were two known strongly

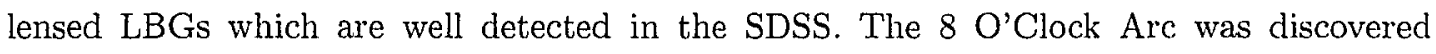
serendipitously from visually inspecting 40,000 images of candidate interacting galaxies. In 
a private conversation Allam suggested that they had several more LBG candidates and that they were easy to find if one knew what they were looking for. Their technique most. likely looks for blue galaxies adjacent to red galaxies. To do this a catalog of pairs of adjacent objects is required. This is not a standard SDSS data product and would be very time consuming to construct properly. As a result their method cannot easily be implemented. However with two objects one can begin to estimate what the properties of the distribution look like. In a last ditch attempt to be absolutely sure no obvious objects were being missed a color cut selection method was developed using the photometry of cB58 and the $8 \mathrm{O}^{\prime}$ Clock Arc. The color cut method was used on the whole sample independently of the regular selection and the cluster correlation methods described above, both of which were also applied to the full data set. Three color different color cut methods were used. All three were applied only objects which survive the SDSS query, flag filter and star rejection discussed above.

The first of these color cuts is created to simply find the objects with colors within the range set by cB58 and the $8 \mathrm{O}^{\prime} \mathrm{Clock}$ Arc. This color cut is shown by the magenta boxes in Figure 4.13. The $8 \mathrm{O}^{\prime}$ Clock Arc is broken up into three pieces by the SDSS photometric pipeline. Each individual piece is plotted in Figure 4.13 in addition to the sum of the three pieces. The boxes are defined by

$$
\begin{gathered}
u-g \geq 1.34 \\
0.11 \leq g-r \leq 0.41 \\
0.01 \leq r-i \leq 0.18 \\
0.20 \leq i-z \leq 0.32
\end{gathered}
$$


where the colors determined from model magnitudes which have been corrected for Milky Way reddening. There is no addition apparent magnitude requirement with this color cut. This cut ensures that no objects with colors identical to cB58 or the sum of the $8 \mathrm{O}^{\prime}$ Clock Arc are missed, nor those with similar colors.

The motivation for the other two color cuts was to develop of cut that the pieces of the $80^{\prime}$ Clock Arc could survive individually. Figure 4.13 shows that the pieces of the 8 O'Clock Arc predominantly fall outside of the magenta box of the color cut described above. This fact highlights the overall problem. All three pieces are images of the same object and should have identical colors. However due to random errors due to their low fluxes they do not. Each of the plus signs in Figure 4.13 is a real LBG, no less the same object, but appears quite different.

The second color cut, the dotted red line in Figure 4.13, is defined as follows:

$$
\begin{gathered}
u-g \geq 2.00 \\
g-r \leq 0.41 \\
g \leq 20.5
\end{gathered}
$$

This cut includes objects with extremely red $u-g$ colors which are also blue in $g-r$. At low fluxes there are a lot of objects with extremely red colors in $u-g$ and $g-r$. These objects are largely red ellipticals with spurious photometry. Here the $g$ band magnitude cut is extremely important in order to have a robust $u-g$ color.

The third color cut, shown by the green line in Figure 4.13, is defined by

$$
1.20 \leq u-g \leq 2.00
$$




$$
\begin{gathered}
g-r \leq 0.20 \\
g \leq 20.9
\end{gathered}
$$

By looking at objects which are bluer in $u-g$ the $g-r$ requirement has to be made more stringent in order to reduce contamination by the galactic locus. On the other hand the $g$ band requirement can be loosened and still have equally robust $u-g$ colors.

The image of every object meeting the criteria of one or more of the three color cuts in addition to the general requirements of the current selection method was individually inspected by eye. 


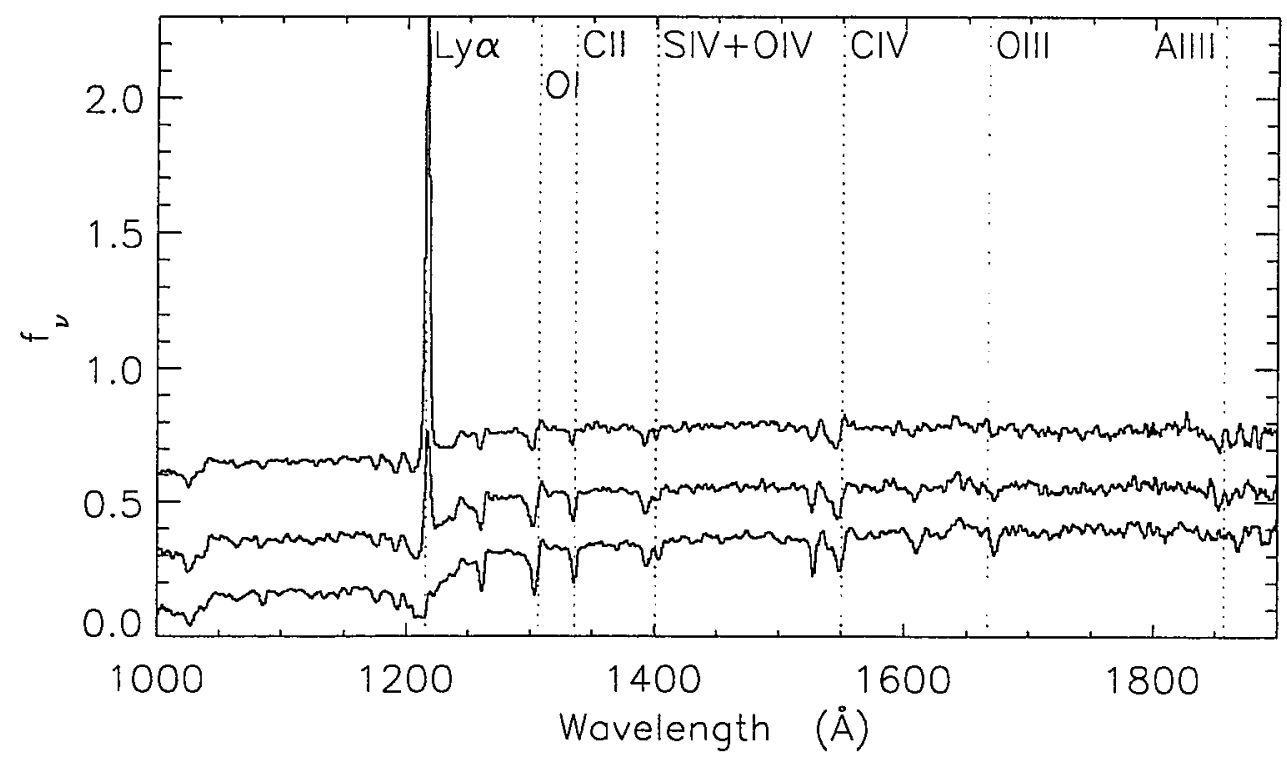

Figure 4.1 Composite Spectra of $z \sim 3$ LBGs from Shapley, et al. (2003). The $f_{\nu}$ flux is in arbitrary units and the top two spectra have been offset for clearer viewing. The 811 individual spectra have been split into quartiles by Ly $\alpha$ equivalent width and added together. The composite spectra of three quartiles is shown here. The quartile with the strongest Lyo emission is omitted. The positions of selected ultraviolet lines are plotted as red, vertical, dotted lines and labeled accordingly. 


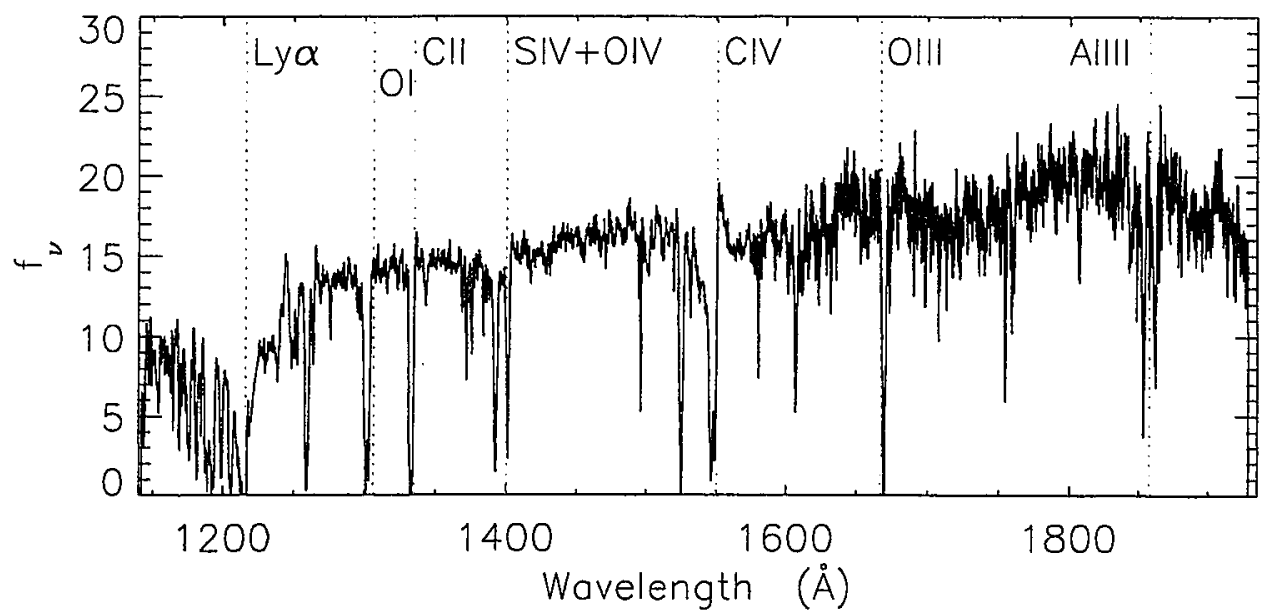

Figure 4.2 The rest-frame ultraviolet spectrum of cB58 from Pettini, et al. (2000). The $f_{\nu}$ flux is in arbitrary units. The positions of selected ultraviolet lines are plotted as red, vertical, dotted lines and labeled accordingly. 


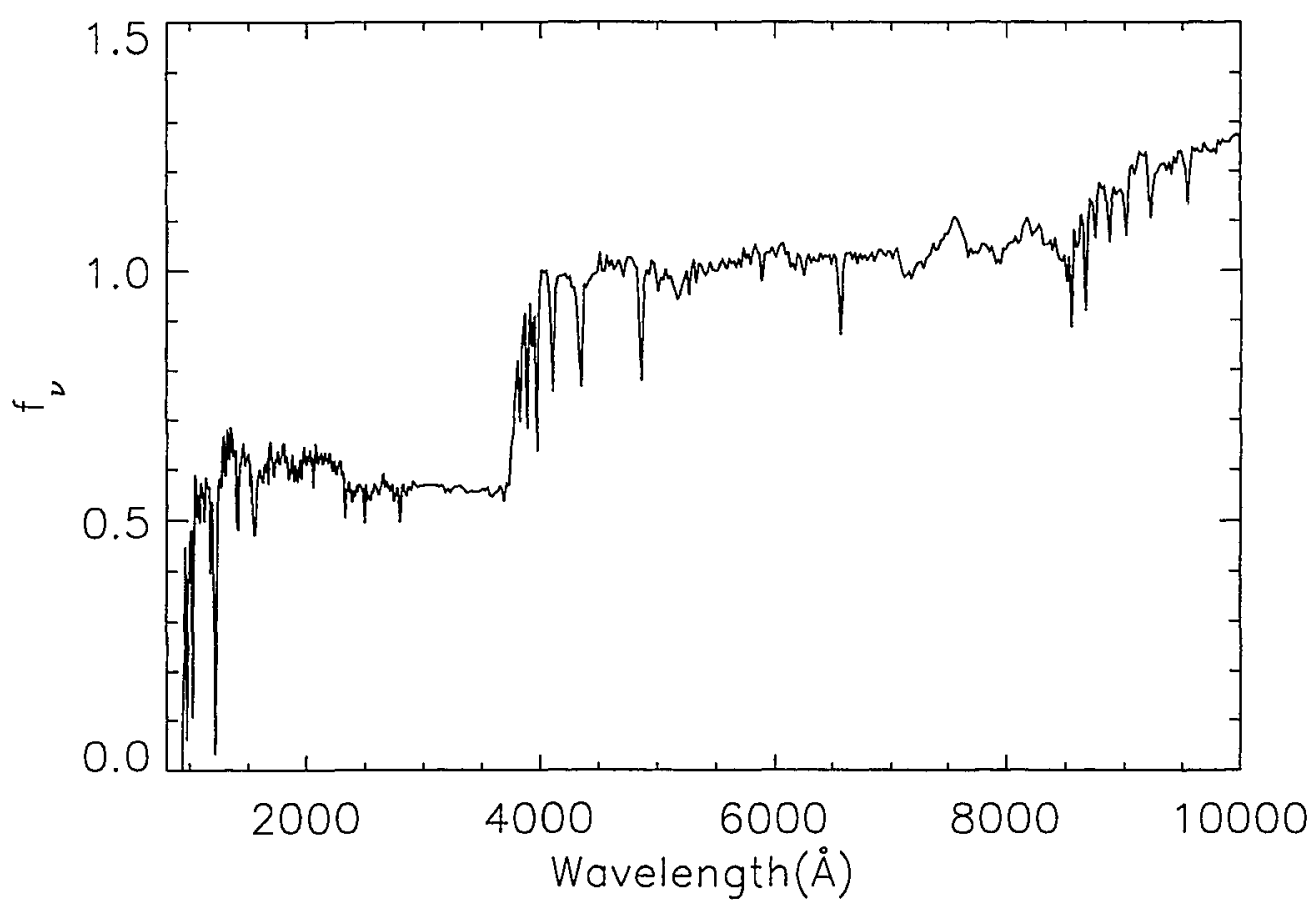

Figure 4.3 Model LBG spectrum from the $912 \AA$ break to the near-infrared with flux in $f_{\nu}$. 


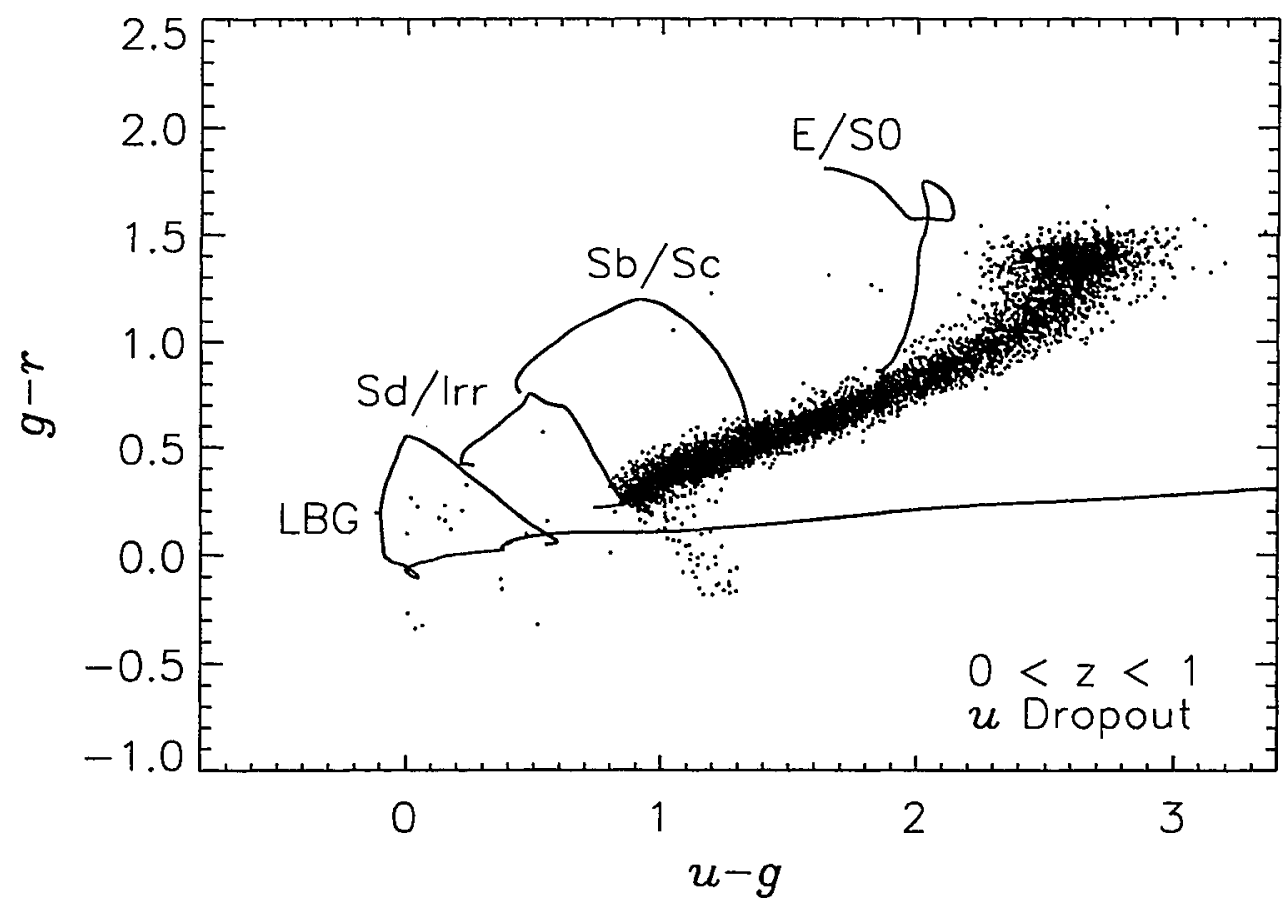

Figure 4.4 LBG track in $u-g$ vs. $g-r$ color space. The blue lines indicate the path of elliptical/S0, Sb/Sc, Sd/Irr and intense star forming (LBG) galaxy models through $u-g$ vs. $g-r$ color space from $z=0$ (lower right end of track for all galaxies) to $z=0$. The cyan line shows the LBG track for $1.0<z<2.29$. The green line shows the LBG track for $u$-band dropouts where $2.29<z<3.17$ where the $912 \AA$ break is passing through the $u$ filter. The black dots are 6,310 point sources from a $20 \mathrm{deg}^{2}$ area on the sky and show the position of the stellar locus with possible contamination by quasars. 


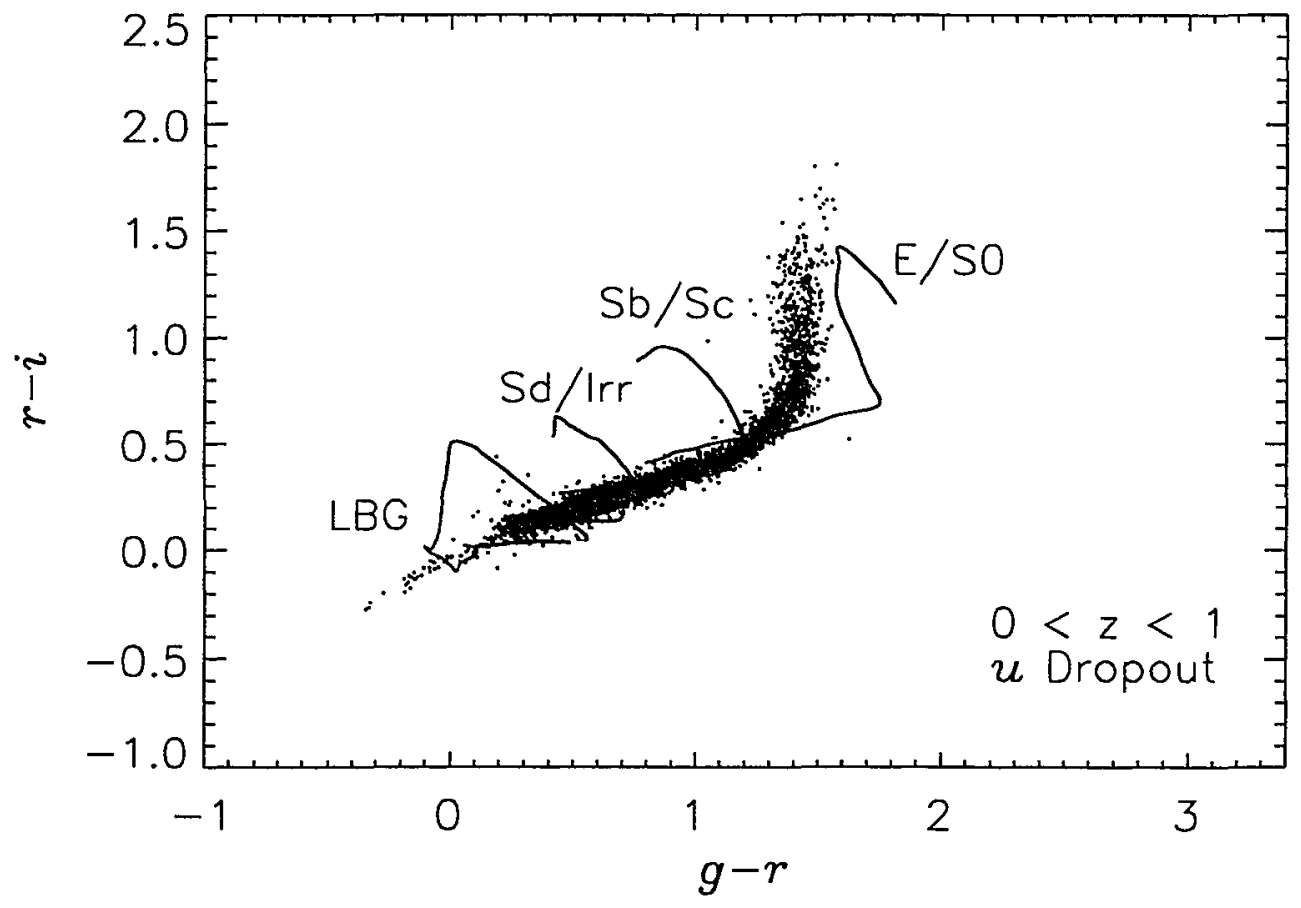

Figure 4.5 LBG track in $g-r$ vs. $r-i$ color space. The description is the same as the same as for Figure 4.4 with the addition of the orange line which denotes $g$-band dropouts where $3.17<z<4.92$. 


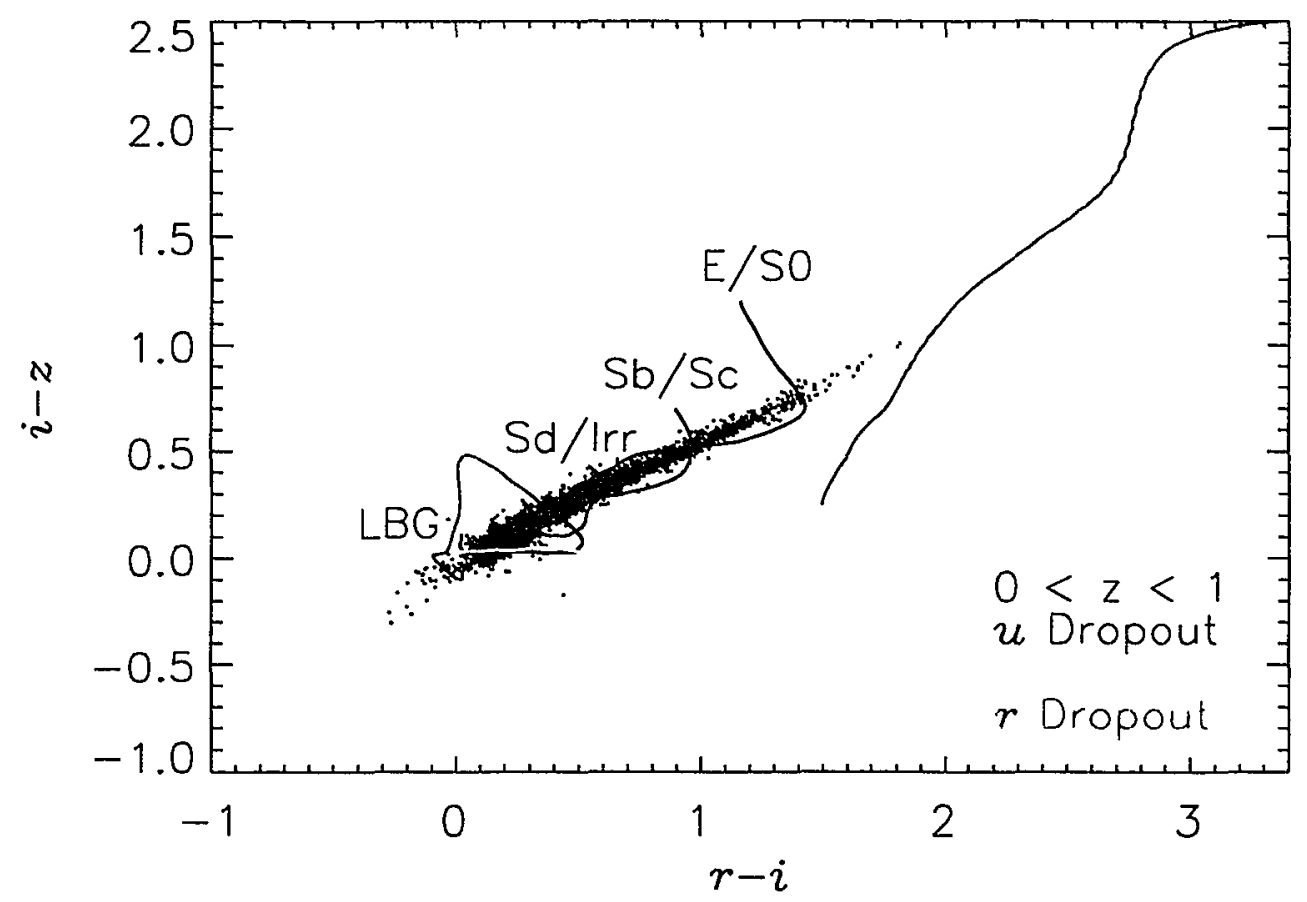

Figure 4.6 LBG track in $r-i$ vs. $i-z$ color space. The discription follows from Figure 4.5 which shows the redshift range of the LBG $r$-band dropouts where $4.92<z<6.34$. 


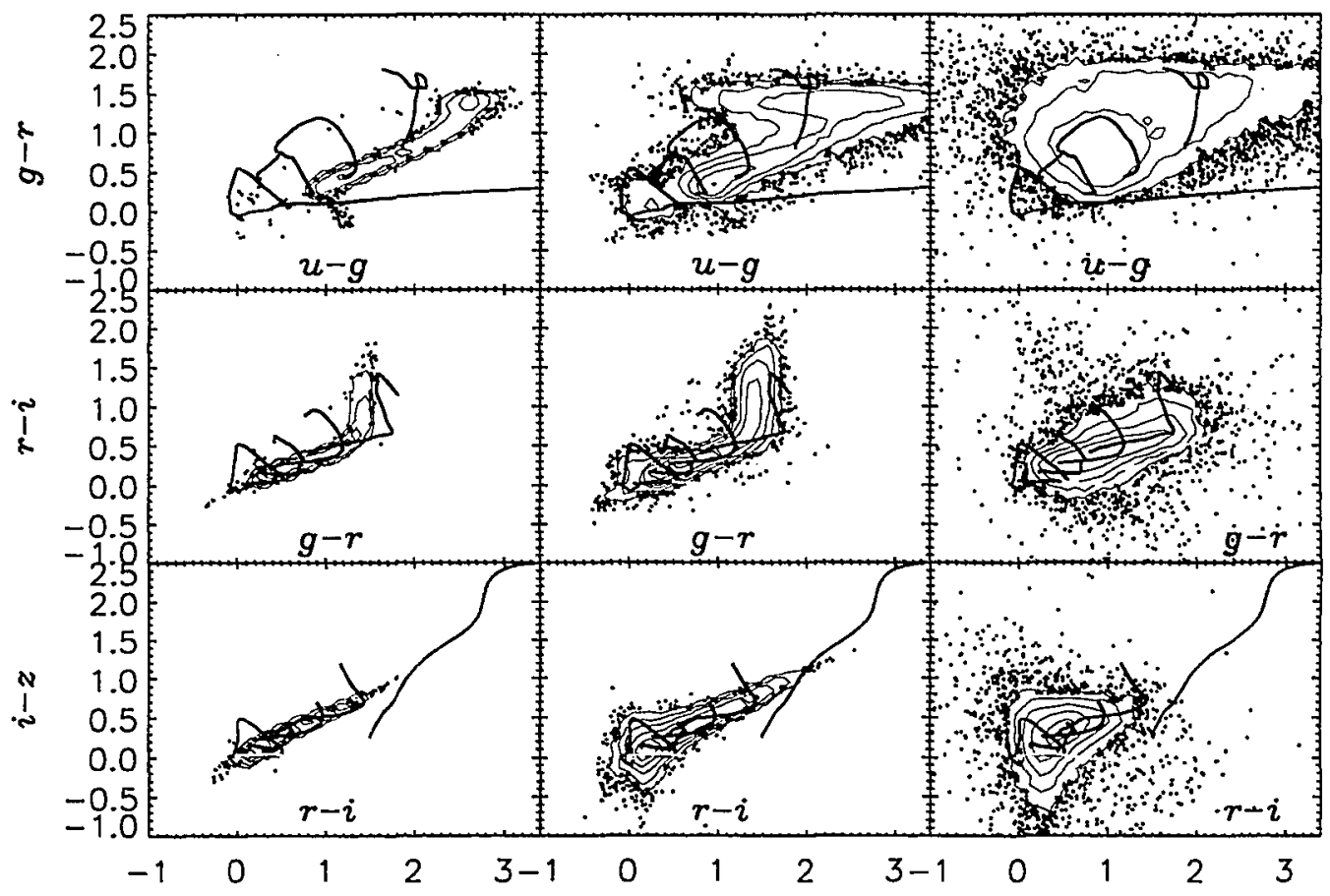

Figure 4.7 Typical scatter in SDSS photometry data in color space. The first column is identical to Figures $4.4,4.5$, and 4.6 except where the density of points is high they have been replaced by logarithmic contours. These are all 6,310 point sources in a $20 \mathrm{deg}^{2}$ area with $15<r<18$ which survive a simple flag filter described in the text. In column two 29,007 point sources with $18<r<21$ from the same region which survive the flag filter are plotted. Column three shows 28,988 extended sources from the same region which survive the flag filter and have $18<r<21$. The top row shows the distribution of the three populations in $u-g$ vs. $g-r, g-r$ vs. $r-i$, and $r-i$ vs. $i-z$ color space in rows one, two and three. For descriptions of the tracks refer back to Figures 4.4, 4.5, and 4.6. 

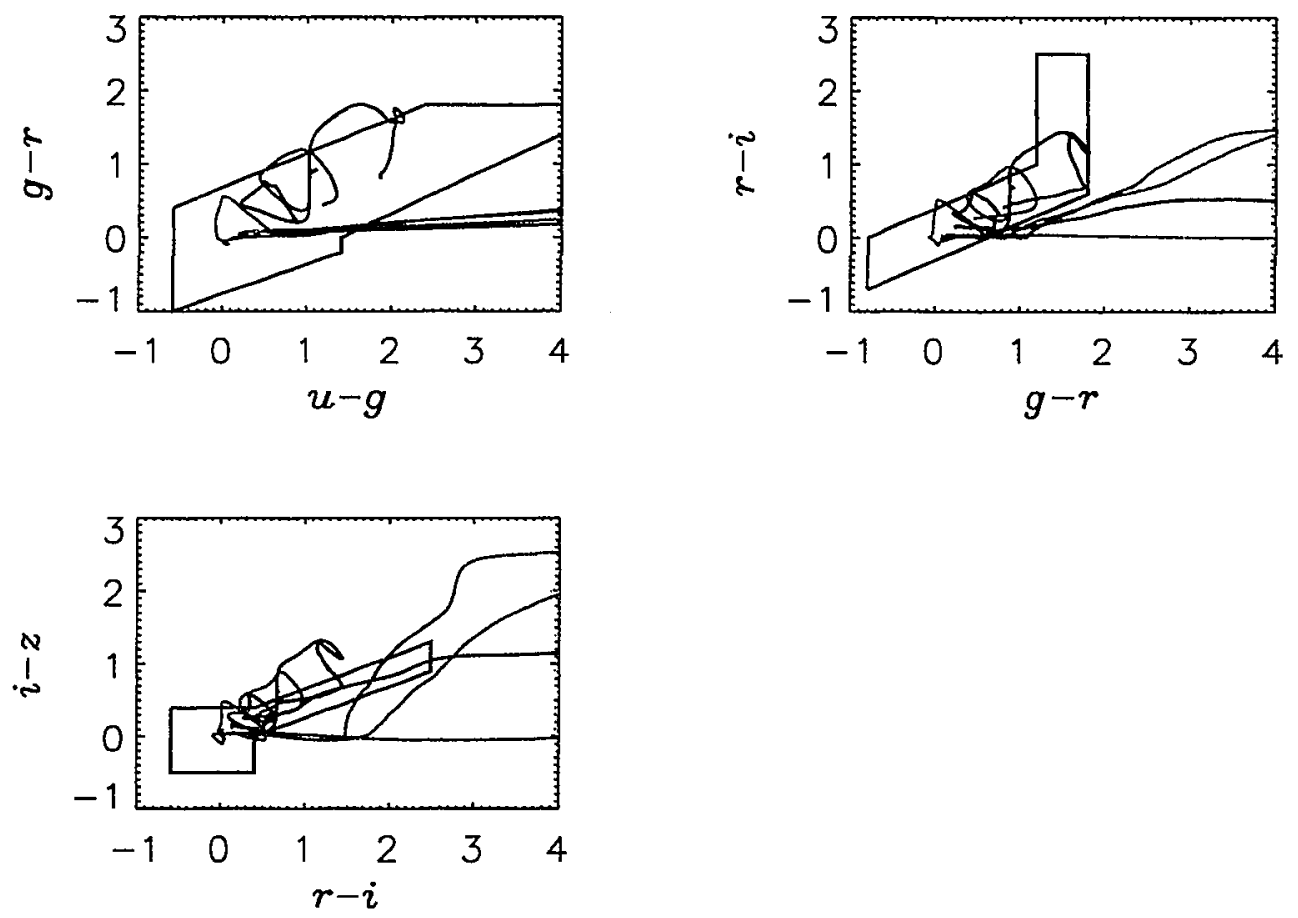

Figure 4.8 LBG selection color cuts from the first generation selection algorithm. The solid black lines, defined in equations $4.8,4.9$ and 4.10, show the stellar locus regions used in the algorithm. The hatched orange regions show the regions for LBG selection as defined in equations 4.6 and 4.7. The green lines show the tracks of the elliptical/S0, $\mathrm{Sb} / \mathrm{Sc}$ and Sd/irregular galaxies, similar to Figures $4.4,4.5$, and 4.6 , for $0<z<10$. The remaining lines show the tracks for the LBG model spectrum of 4.3 given a range of IGM extinction models: $10 \%$ (blue), median (cyan), mean (red) and 90\% (magenta). 


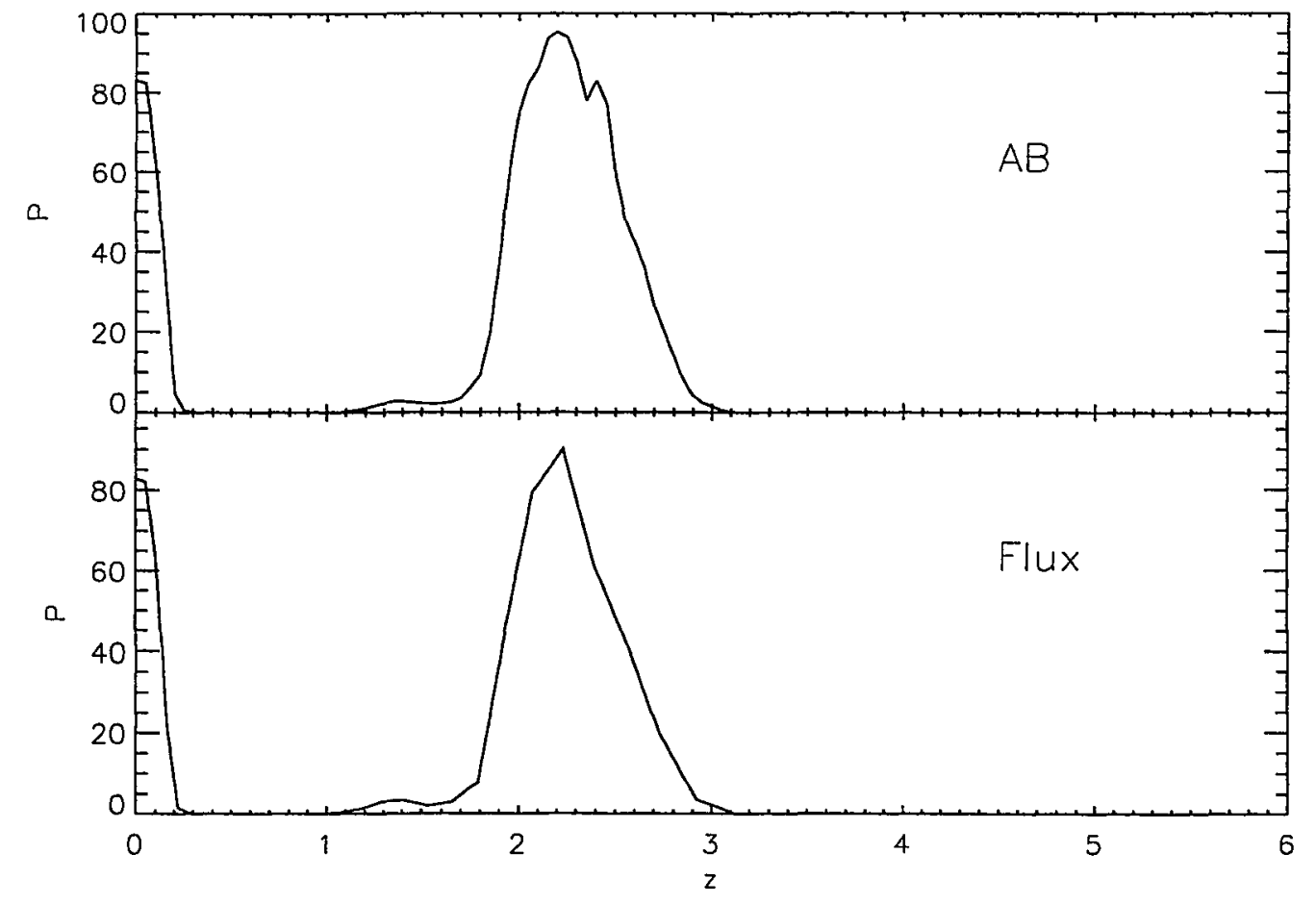

Figure 4.9 Hyperz results for cB58 with and without flux correction modification. The plot shows the probability that $\mathrm{cB} 58$ is at a given redshift. In the top panel is the result, of the original method using Hyperz "as is," approximating the SDSS luptitudes by AB magnitudes. In the bottom panel the current results are shown using the modified version of Typerz which treats the SDSS luptiludes properly. The resolution in redshift in the new results has been lowered in order to increase the range of extinction models tested. 


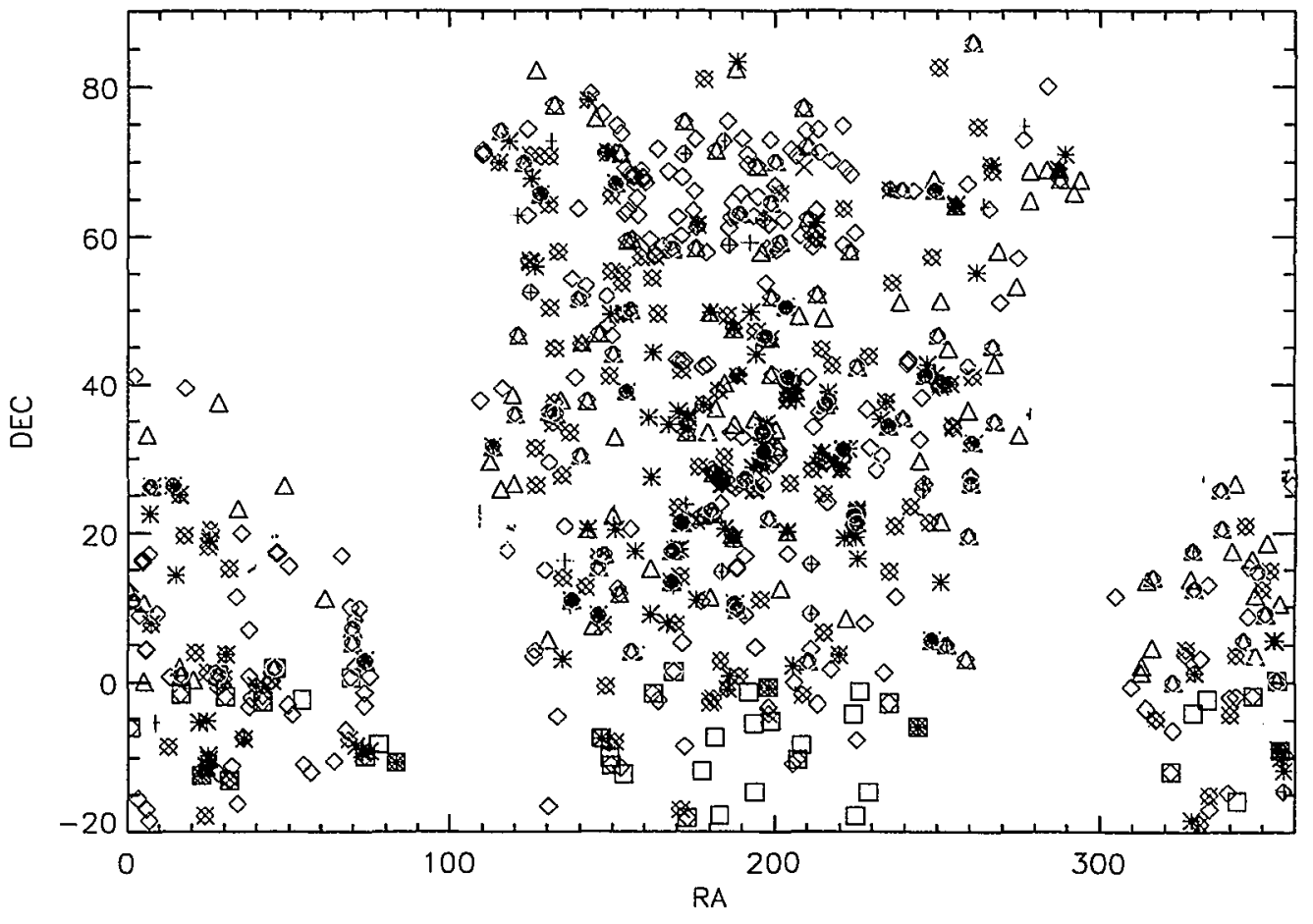

Figure 4.10 Positions of known galaxy clusters in equatorial coordinates. Objects with declinations below $-20^{\circ}$ are not plotted due to the fact that these objects do not overlap with the SDSS. Galaxy clusters are plotted by cluster catalog. The catalogs are the Abell rich cluster catalog (brown asterisks), FSVS catalog (magenta points), Einstein catalog (red crosses), RASS brightest cluster catalog (gold circles), REFLEX catalog (blue squares), NORAS catalog (green triangles), ROSAT PSPC catalog (cyan x's), and the BAX catalog (black diamonds). 


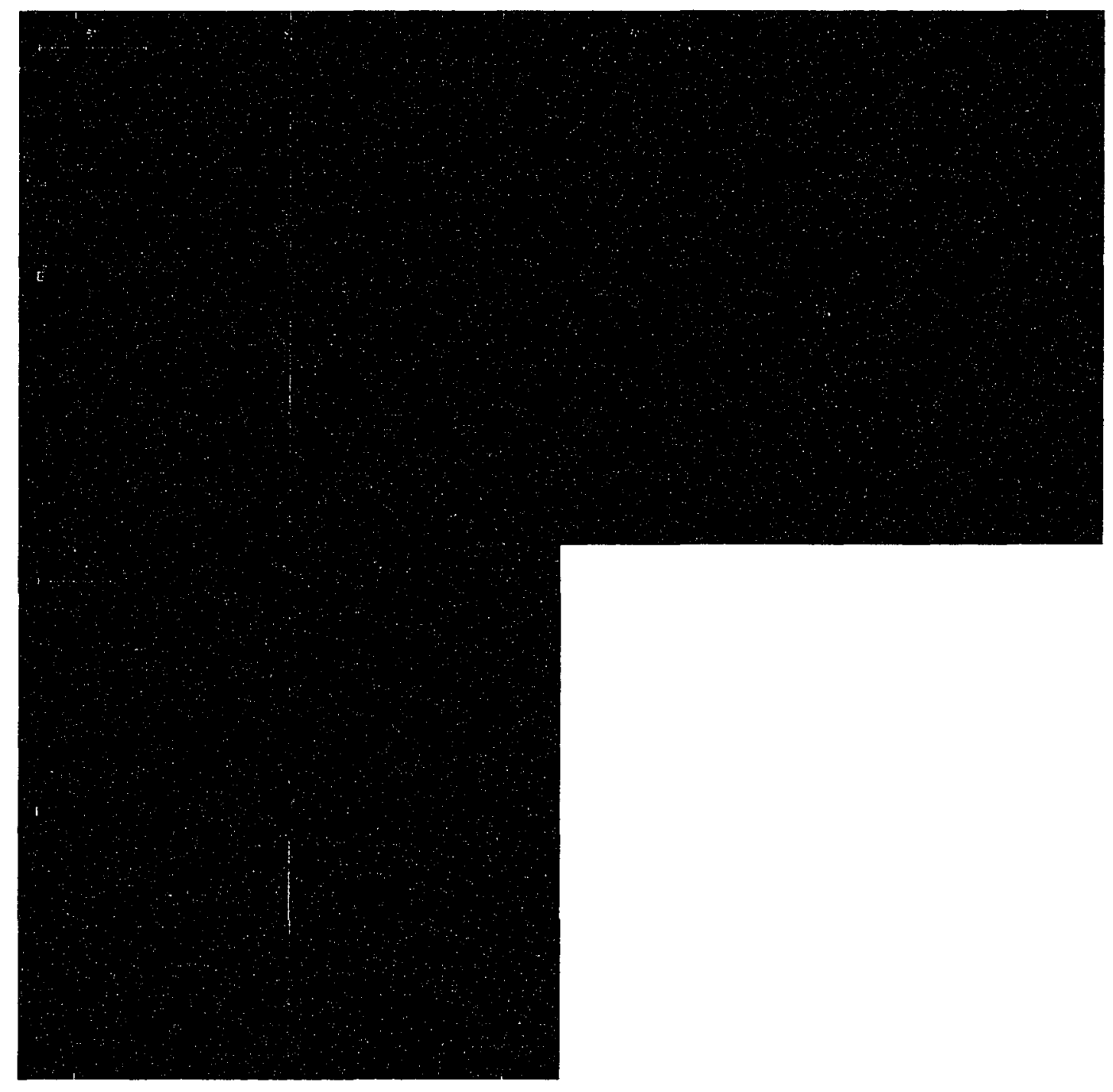

Figure 4.11 SDSS images of known strongly lensed LBGs. Upper left: MS1512-cB58. Upper right: The Cosmic Eye, LBG J213512.73-010143. Lower left: The 8 O'Clock Arc, SDSS J002240.91+143110.4. 


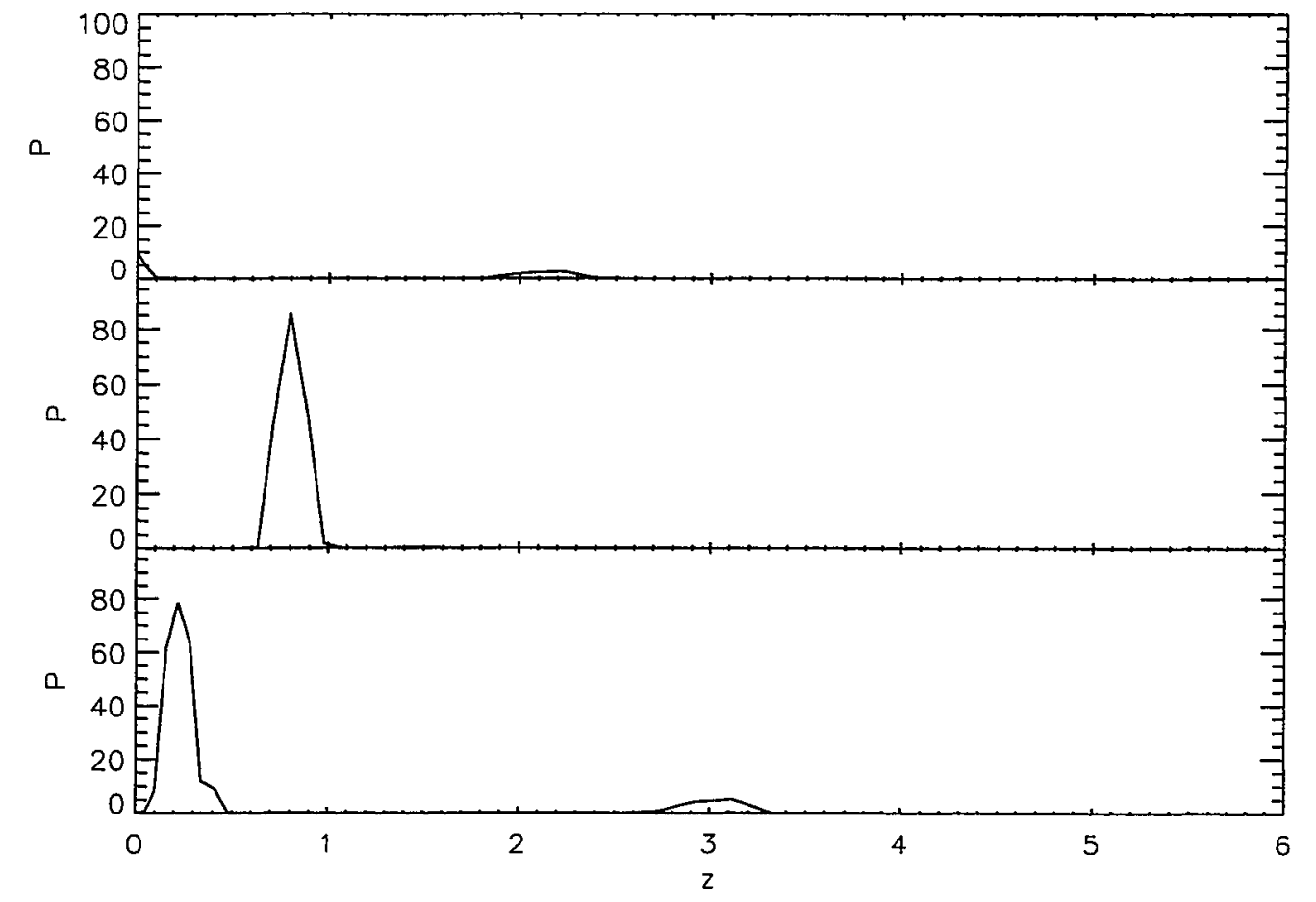

Figure 4.12 Photometric redshifts of targeted galaxies. The plots show the probability, as calculated by the modified version of Hyperz, that the galaxy in question is at a given redshift. The top panel is for SDSS J1547+2056, the middle panel is for SDSS J1640+4643, and the bottom panel is for a galaxy which survives all of the criteria of the current selection along the same line of sight as SDSS J1640+4643. 

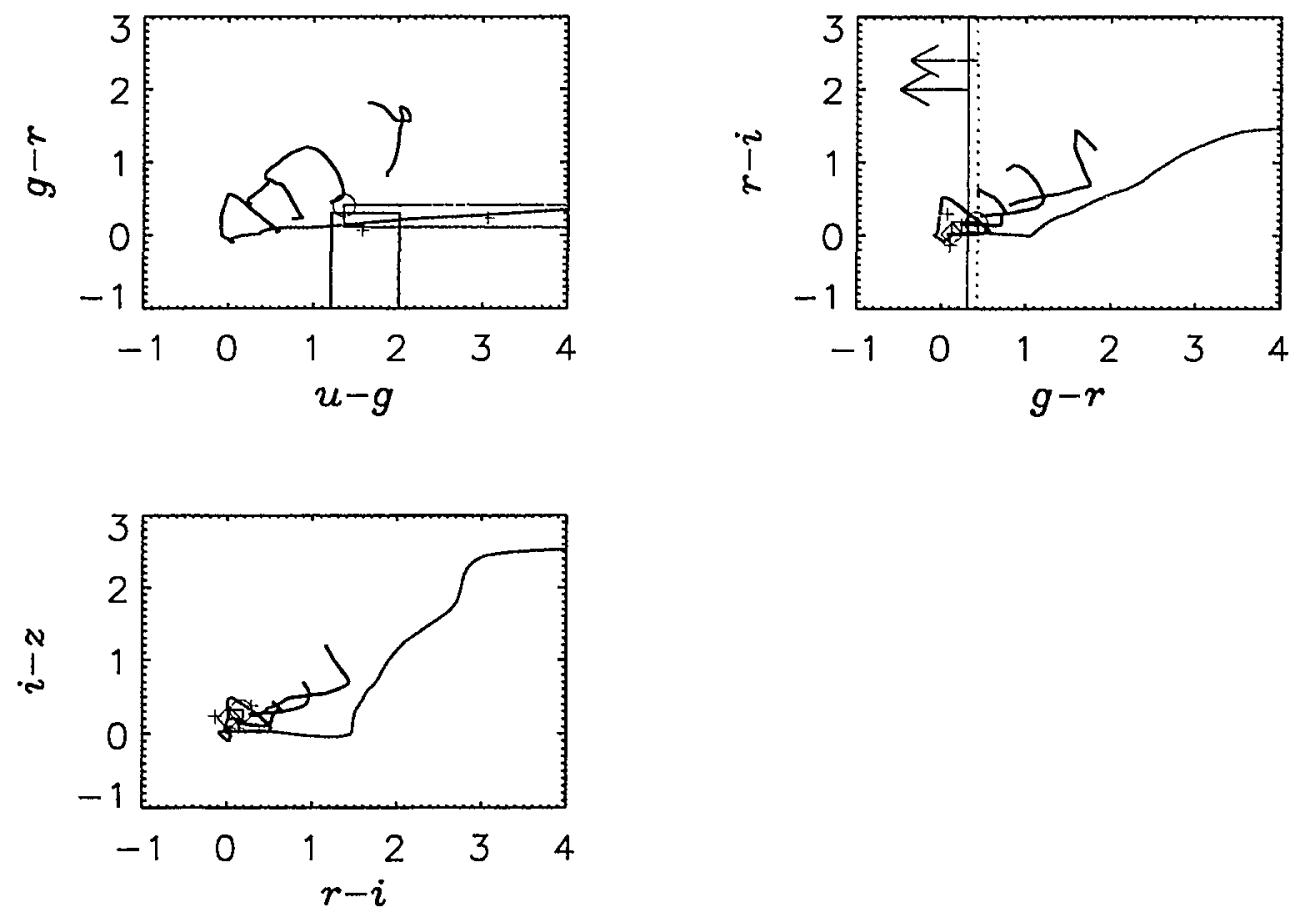

Figure 4.13 Current LBG color cut based on SDSS photometry of cB58 and the 8 O'Clock Arc. The blue lines are galaxy model tracks with $z \leq 1.0$. The cyan lines are the LBG tracks for $z>1.0$. The the positions of the known LBGs are given by a circle (cB58) and a diamond (sum of the three 8 O'Clock Arc pieces). The plus signs show the positions of the individual pieces of the $8 \mathrm{O}^{\prime}$ Clock Arc. The three color cuts described in the text are given by the magenta and green solid lines and the red dotted line. The arrows in the upper left panel indicate that there is no blue bound to the $g-r$ color. 


\section{Chapter 5}

\section{Spectroscopic Study of Lensed Lyman Break Galaxy Candidates}

\subsection{Spectroscopic Results}

In the previous chapter the algorithms for targeting lensed LBGs were discussed. In this chapter the results of the spectroscopic follow-up of these objects are discussed. In addition several secondary searches for lensed LBGs are discussed.

\subsubsection{First Generation Algorithm}

\section{Observations}

Targets generated by the first generation algorithm were observed primarily with the $2 \mathrm{dF}$ instrument on the $3.9 \mathrm{~m}$ Anglo Australian Telescope. $2 \mathrm{dF}$ is a multi fiber spectrograph instrument capable of observing 400 spectra at a time wilhin a two degree field of view (Lewis, et al. 2002). This project was run piggy backed on a project to measure the level of quasar activity in the universe at $z \sim 3$ which is the period in cosmic history 
at which quasars were most active (Chiu 2006). Out of the $4002 \mathrm{dF}$ fibers around ten per pointing were used for this project.

$2 \mathrm{dF}$ is a unique and imnovative spectrograph. Fiber optic cables are attached by magnets to metal plate at the prime focus of the telescope. The location of the fibers in the field of view is such that the light from objects of interest falls on the ends of the fibers. The light from an object passes through the fiber optic cables into one of two spectrographs. The 400 fibers are connected around the edge of the circular plate and point radially inward when configured. The fibers are kept taut so as not to get tangled and block other fibers. Specialized software determines the optimal locations of the fibers. There are a few constraints. One is that there is a physical limit to how close the fibers can be placed to each other due to the size of the magnet which affixes them to the plate. Secondly the fibers are not allowed to cross each other. If an object is assigned to a specific spectrograph this is an additional case. In the standard case both spectrographs are set up with the same gratings. For the quasar project one spectrograph had a blue grating (300B)for objects targeted as $z \sim 3$ quasars and a red grating (316R) for objects targeted as $z \sim 5$ quasars. Due to these constraints the target list is larger than the number of fibers and the software then allocates the fibers in the most effective way possible.

The other unique feature is that the fibers are placed in an automated fashion using a robotic arm. The $2 \mathrm{dF}$ instrument has fiber assemblies on two sides. While one side is being used for obscrvations the fibers can be rearranged on the other end. When switching from one observational ficld to the next the whole instrument is rotated $180^{\circ}$ so that new observations can begin immediately. 
As mentioned earlier the first attempt to find lensed LBGs was largely an opportunistic use of unallocated fibers from the quasar survey. The density of targets generated by the quasar survey in a given field dictated how many fibers went to LBG targets. The fiber allocation software also influenced the number of LBG targets observed per field, depending on fiber collisions.

In addition to the science targets around 4 fibers per pointing were used for guide stars and around 30 were used to measure the sky. The exposure time used was 15 minutes and four to five exposures were taken per field depending on the conditions. Flat field and arc lamp exposures were also taken. Data reduction was done using the $2 \mathrm{dfdr}$ software, which was designed specifically for the $2 \mathrm{dF}$ instrument, in the standard way.

A full list of all LBG candidates observed can be found in Appendix A. Most of the targets observed were too noisy to identify. The goal of the quasar study was simply to identify quasars and measure their redshifts. Quasar spectra have strong, broad emission lines which are fairly easy to identify even with poor S/N. Galaxies on the other hand tend to have narrow emission lines and many, including LBGs like $\mathrm{cB58}$, do not have any prominent emission lines. However the observing strategy was set solely with the quasar study in mind. As a result galaxies with magnitudes similar to those of the quasars found can be too noisy to identify. Many of the targets had stellar type spectra.

However two objects targeted as LBG candidates were clearly identified as quasars. Their spectra can be found in Figure 5.1. SDSS J145121.82+010811.8 has a redshift of $z=3.35$ and SDSS J001714.67-100055.5 has a redshift of $z=5.03$. SDSS J0017-1000 was at the time the most distant object ever discovered using the AAT. 


\section{Analysis of Algorithm Shortcomings}

Due to the constraint of observing as part of another program of higher priority the first generation algorithm suffered in part from outside forces. The algorithm was never used to identify the absolute "best" targets on the sky as defined by its own criteria. Rather a region of $\pi$ square degrees was determined by others and the ten or so most likely candidates were identified and observed. As such the algorithm was never fully tested on its own merits. However, given the objects observed some lessons were learned.

On the bright side two quasars were found with redshifts $z \sim 3$ and $\sim 5$. In this sense the algorithm is a partial success as it managed to identify bona fide members of the high redshift universe. Quasars drop out of photometric filters for the same reason that galaxies do. It is reassuring that drop outs, albeit not galaxies, are found by this algorithm.

One of the big lessons learned was that several objects that were identified as resolved sources by the SDSS photometry were actually stars. This was in addition to the stellar spectra from objects which were identified as point sources in the photometry. It was clear that it if the observations were conducted in a more targeted way the level of stellar contaminants was far too high. This led to the decision to revise the algorithm to reject unresolved sources as well as to be more strict with the star-galaxy separation than the SDSS pipeline.

Another problem is that the galaxy rejection method clearly does not cover the whole of the galactic locus. With only three tracks being used for fitting a well detected Sa galaxy can easily fall in the gap between the $\mathrm{E} / \mathrm{S} 0$ and $\mathrm{Sb} / \mathrm{Sc}$ galaxy tracks and survive the galaxy rejection. 


\subsubsection{Second Generation Algorithm}

\section{Observations}

The second generation algorithm was used for observations in the second half of 2002. In total 7 objects were observed with the $2 \mathrm{dF}$ instrument and 3 were observed using the Faint Object Camera and Spectrograph (FOCAS, citetKashikawa02)) instrument on the $8 \mathrm{~m}$ Subaru Telescope. This is far fewer than the numbers observed in the July 2001 and May 2002.

The $72 \mathrm{dF}$ objects were observed and analyzed in the same way as the earlier objects. These observations are detailed in the previous section.

The observations with FOCAS were done in the long slit spectroscopy mode where only one object was observed at at time. Given the large $8 \mathrm{~m}$ aperture of the Subaru Telescope and the sensitivity of FOCAS only one $300 \mathrm{~s}$ exposure was taken for each object. These spectra were reduced using the apextract package in IRAF. These spectra were all well detected so the reduction was carried out in the standard way.

The FOCAS spectra are shown in Figure 5.2. The objects were revealed to be an A star and two nearby galaxies with redshifts $z \sim 0.02$. The full list of observations is shown if Table A.1. Of the ten objects targeted using the second generation algorithm four are determined to be emission line galaxies.

\section{Analysis of Algorithm Shortcomings}

While the problem of contamination by individual stars seems to be resolved by the second generation algorithm, binary stars with small separations are revealed to be a 
source of contamination. SDSS J020655.21-005817.4 is classified as a star by the SDSS imaging star/galaxy separator. The left half of Figure 5.3 shows the SDSS image of SDSS J0206-0058 which appears to be extended. This object was observed spectroscopically using the FOCAS instrument on the Subaru Telescope, an $8.2 \mathrm{~m}$ telescope at the summit of Mauna Kea. A quick exposure, seen on the right half of Figure 5.3, made for slit placement clearly shows that the object is actually two point sources with a small separation. The spectrum, in Figure 5.2 reveals that the brighter object is an A type star. The SDSS colors of the object do not appear to be stellar because it is actually two stars summed together. Such pairings of stars have the potential to have strange colors which can confuse the algorithm. Another significant problem with the second generation algorithm is that it picks out low redshift emission line galaxies. This is particularly troubling. These objects should be identified by HyperZ and rejected.

\subsubsection{Current Algorithm}

\section{Observations}

The observations for targets selected by the current algorithm were all conducted using the Dual Imaging Spectrograph (DIS III) on the $3.5 \mathrm{~m}$ ARC Telescope at Apache Point Observatory. These observations were conducted remotely from both Baltimore, Maryland and Melbourne, Australia. The observations were conducted from October 2006 to June 2007. DIS III was used in long slit spectroscopy mode. Depending on the nature of the targets and the weather conditions exposure times ranged from 30 to 75 minutes. The spectra were reduced using the apextract package in IRAF. 
In total 14 objects selected with the current algorithm were observecl, 8 of which are clearly identified. The list of observed objects can be found in Table A.1. Selected spectra from these observations are shown in Figures 5.4, 5.5, and 5.6.

Five of the eight galaxies are identified as having redshifts below $z=1$ by weak emission lines. Figure 5.4 is an example of one of these galaxies. Two of these galaxies have $\mathrm{H} \beta$ lines which are far weaker than expected given the observed strengths of the $\mathrm{H} \alpha$ line. Figure 5.4 is an example of this. The expected ratio of $\mathrm{H} \alpha$ to $\mathrm{H} \beta$ is around 2.8 and is discussed at greater length in Chapter 2. Larger values of this ratio are due to extinction within the galaxies. The low ratio seen in Figure 5.4 points to very heavy extinction which makes the galaxy appear much redder than it should be given its stellar population. This could be the reason why the modified version of HyperZ fails to reject it.

One of the eight objects (Figure 5.5) is a galaxy with a pronounced Balmer break. The last two contaminants, one of which is found in Figure 5.6, have quasar spectra. These objects are interesting in that they are both truly extended as evidenced by the SDSS imaging and the the width of the trace in the long slit spectroscopy image. One possible explanation for this is that they are lens systems, like the one in Figure 1.4, where the separation of the images is too small to the images to be resolved by SDSS. The result mimics an extended object.

\section{Analysis of Algorithm Shortcomings}

The current algorithm is a clear step forward. For one the emission line galaxies have largely been eradicated. The current emission line galaxy contaminants have much weaker emission lines and are much closer to being continuum sources. Another contaminant 
is a Balmer break galaxy. The Balmer break can be difficult to tell from the Lyman break using photometric redshift codes. This is a contaminant one likely has to live with. In addition several high redshift quasars have been found. These are drop out objects like the LBGs so this is at least a step in the right direction. Generally quasars are point sources and should be easily rejected among the first steps of the algorithm. However inspections of the SDSS images and the long slit spectroscopy images indicate that these truly are extended objects. It is possible that this algorithm is useful for picking out new quasar lens systems. While that is something of a mystery it bodes well for the selection algorithm as these are truly unusual objects. Another contaminant is heavily extincted emission line galaxies. Again, these are another kind of unusual object. The algorithm does appear successful for the most part at rejecting mundane objects. Given that this algorithm was specifically designed to find cB58 any object similar to $\mathrm{cB} 58$ will be found. Given that none of these objects were detected LBGs must be difficult to find photometrically in the SDSS. At this point the only way to improve this algorithm seems to be to have a larger set of known lensed LBGs as a point of reference.

\subsection{Mining Known LBGs from the SDSS}

At the beginning of the LBG search there were no known strongly lensed LBG candidates within the SDSS footprint. As a result in the early stages of the project it was not possible to test selection methods against actual objects. There was a complete reliance on models. However at present three of the four known strongly lensed LBGs have been observed by the SDSS. Each of these objects provides a valuable opportunity to check the 
Table 5.1. SDSS Photometric Data for Known Strongly Lensed LBGs

\begin{tabular}{|c|c|c|c|c|c|}
\hline & \multirow[t]{2}{*}{ cB58 } & \multicolumn{4}{|c|}{$80^{\prime}$ Clock Arc } \\
\hline & & Sum & $A$ & B & $\mathrm{C}$ \\
\hline$u$ & $22.38 \pm 0.30$ & $24.62 \pm 3.10$ & $25.73 \pm 1.69$ & $23.41 \pm 0.71$ & $24.83 \pm 1.10$ \\
\hline$g$ & $21.00 \pm 0.04$ & $19.99 \pm 0.04$ & $20.55 \pm 0.05$ & $21.77 \pm 0.07$ & $21.69 \pm 0.00$ \\
\hline$r$ & $20.58 \pm 0.04$ & $19.81 \pm 0.05$ & $20.39 \pm 0.07$ & $21.64 \pm 0.09$ & $21.47 \pm 0.07$ \\
\hline$i$ & $20.39 \pm 0.05$ & $19.75 \pm 0.06$ & $20.49 \pm 0.10$ & $21.31 \pm 0.09$ & $21.23 \pm 0.07$ \\
\hline$z$ & $20.07 \pm 0.12$ & $19.50 \pm 0.19$ & $20.22 \pm 0.31$ & $20.89 \pm 0.24$ & $21.11 \pm 0.27$ \\
\hline$u-g$ & 1.35 & 4.56 & 5.11 & 1.57 & 3.06 \\
\hline$g-r$ & 0.40 & 0.12 & 0.10 & 0.07 & 0.23 \\
\hline$r-i$ & 0.17 & 0.02 & -0.14 & 0.29 & 0.14 \\
\hline$i-z$ & 0.31 & 0.21 & 0.23 & 0.38 & 0.08 \\
\hline
\end{tabular}

selection method to determine if it is capable of finding real LBGs and insight on how to improve the criteria for increased efficiency. In this section each of the known LBGs is discussed in the context of whether or not the selection methods identify the object and if not if an improvement of the rubric could potentially rectify the deficiency.

\subsubsection{MS1512-cB58}

MS1512-cB58, the first strongly lensed LBG found, was discovered serendipitously in a galaxy cluster redshift survey. It is the inspiration for this project to find strongly lensed LBGs with a systematic approach using photometry data. As mentioned earlier the current selection algorithm was designed explicitly such that cB58 would pass. It is the first of the three algorithms described to have the benefit of hindsight. It was not until after the 
first two algorithms were implemented that the SDSS finally observed cB58.

The first generation algorithm has major problems when it comes to finding cB58.

First off the CHILD flag is set for cB58. Secondly cB58 falls within all three star boxes in. Figure 4.8 and as a result would be rejected as a star. Lastly, cB58 falls outside the orange LBG selection box in Figure 4.8 and would be rejected on those grounds as well.

\subsubsection{The Bullet Cluster}

The LBG in the Bullet Cluster was discovered by a chain of events. First the cluster was identified as an extended X-ray source by the space-based Einstein X-ray telescope during its lifetime between 1978 and 1981. Tucker, Tananbaum, \& Remillard (1995) did CCD imaging of the field and discovered the cluster as well as a 12" luminous arc. Mehlert, et al. (2001) spectroscopically confirmed the object as an LBG.

With a declination of $-56^{\circ}$ the Bullet Cluster is outside of the SDSS footprint. As such there was no chance of finding it in this project. However it is doubtful that it conld have been pulled out of the SDSS. For one, the object has an apparent magnitude of $R=22$. This is well below the limit where the SDSS photometry is reliable. The Mehlert, et al. (2001) image is from the VLT and has 0.80 " seeing in the $R$ band which is considerably better than the median SDSS seeing of 1.43". The LBG has a length of 14 " and width of 1.8 " so the surface brightness is low. It is also located 2" from a brighter elliptical galaxy which would almost certainly result in spurious photometry after deblending. The other four high redshift objects in the field are even fainter and would be impossible to find in the SDSS.

The Bullet Cluster LBG was discovered serendipitously and is too faint to be 
systematically identified in the SDSS.

\subsubsection{The Cosmic Eye}

The Cosmic Eye was discovered in Hubble Space Telescope imaging as part of a program to find bright lensed galaxies in X-ray luminous galaxy clusters. The Hubble ACS image in Smail, et al. (2007) has very sharp spatial resolution and clearly shows a central elliptical galaxy with two thin arcs forming nearly a full circle around it. However in the SDSS image, shown in Figure 4.11, the arcs and the central elliptical galaxy are blurred together. The Cosmic Eye is bright enough to survive the selection criteria with $r=20.27$ and $i=19.74$, however the light is dominated by the central elliptical galaxy. The colors of the object are $u-g=2.23, g-r=1.15, r-i=0.50$, and $i-z=0.26$. Comparison with Figures $4.4,4.5$, and 4.6 show that these colors are consistent with elliptical galaxies and inconsistent with LBG colors. In addition the Cosmic Eye has the NOPETRO flag set which would be rejected by the current flag filter.

\subsubsection{The 8 O'Clock Arc}

The 8 O'Clock Arc was discovered serendipitously by visual inspection of over 40,000 images of candidate interacting galaxies. It is the only strongly lensed LBG to be discovered using the SDSS data. It was not found by its photometry alone, but primarily by its proximity to another object. The key point about the SDSS observations of the 8 O'Clock Arc is that the SDSS photometric pipeline treats it as three pieces. The details of each individual piece as well as the sum of all three are shown in Table 5.1. The sum is determined by correctly converting the luptitude of each object in each band into flux 
via equation 4.3 , summing the fluxes, and then converting back to luptitudes using eqation 4.1. In the sum the $8 \mathrm{O}^{\prime} \mathrm{Clock}$ Arc is well detected with $r=19.81$. However the individual pieces are fainter with $r$ band magnitudes of $20.39,21.64$, and 21.47 for pieces $\mathrm{A}, \mathrm{B}$, and $\mathrm{C}$ respectively.

The $8 \mathrm{O}^{\prime}$ Clock is in trouble right off the bat in the first generation algorithm. The first generation algorithm has a magnitude cut $i<21$. Piece A survives with $i=20.49$ but pieces $\mathrm{B}$ and $\mathrm{C}$ are both a half magnitude too faint. All three pieces have the CHILD flag set in the global object flags so they would be the $80^{\prime}$ Clock Arc would be completely rejected at the first step. In addition piece A and B and the sum all have $i-z>0.20$ which falls outside of the LBG area in Figure 4.8.

As for the current selection algorithm, pieces $B$ and $C$ are immediately rejected by the requirement that $i<20.5$ and piece $\mathrm{A}$ only makes it by 0.01 magnitudes. Piece $\mathrm{B}$ passes the flag filter but $A$ and $C$ are rejected. Both have the NOPETRO and $C R$ flags set in the $r$ band. Piece $\mathrm{C}$ is also a product of the deblender.

Figure 5.7 gives the photometric redshift results for the full $80^{\prime}$ Clock Arc and the pieces thereof. The photometric redshift results for the full arc are sharply peaked at $z \sim 3.1$ which is slightly higher than the true redshift $z=2.73$. The sum is given only a $\sim 1 \%$ chance of being at the true redshift. Even more impressive is that there are no peaks at low or intermediate redshifts. The probability is zero everywhere except the main high redshift peak. The same is truc, althought the poak is not as sharp for pieces A and C. Piece $\mathrm{B}$ has its strongest peak at $z=0$ and has a broad secondary peak ranging from $z=1$ to 3. The photometric results for pieces $A$ and $B$, and especially the sum, are very promising. 
They are much better than the results for cB58 in Figure 4.9 in that there is no low redshfit peak to cause confusion. Unfortunately this point is academic. The sum, which has the best results, is not in the SDSS database as one needs to know a priori to add the sum the fluxes of the three pieces the arc is divided into by the pipeline. One would already be a long way towards identifying the object as a gravitational lens by that point. The

On the upside this proves that scans of the photometric redshift data looking for: objects with strong high redshift peaks in conjunction with no peaks at low redshift can find objects like the 8 O'Clock Arc if they are in the sample.

\subsection{Other LBG Searches}

In addition to the main photometric search several unrelated opportunities arose to look for lensed LBGs. In this section these projects are described as well as the techniques used to implement them.

\subsection{1 u-band Selected Galaxy Survey}

In the late summer the North Galactic Pole is not observable. During these times the SDSS Southern Survey is in operation which entails repeat imaging and spectroscopy of a relatively small area along the celestial equator in the Southern Galactic cap. In addition to the main survey the SDSS accepts proposals for special spectroscopic observing campaigns. Special plates are drilled for these observations which are reduced in processed using the standard SDSS spectroscopic pipeline.

One such special program is the SDSS $u$-band Galaxy Survey (Baldry, et al. 2005). 
The goal of the $u$-band survey was to measure the galaxy luminosity function in the $u_{0.1}$ band and its evolution with redshift. To accomplish this galaxies were selected by a combination of six programs, designed in particular for different purposes, which when combine yield a sample with $u<20.5$ in Petrosian magnitudes.

In order to improve the $\mathrm{S} / \mathrm{N}$ ratio in the $u$-band photometry the co-added imaging catalog was used. To increase the depth of the imaging individual imaging runs must be added together into combined images. However to improve the $\mathrm{S} / \mathrm{N}$ the imaging catalogs can be added together. Because the SDSS uses luptitudes (\$4.2.1) no flux information is lost, even in the case of a non-detection. The catalog of each observing run can be easily converted back to flux, added to subsequent observations, then converted back into luptitudes. This has the advantage of being easier to do than adding the imaging together, especially since the number of observations differs from region to region and because the seeing can be different from night to night.

We searched the $u$-band selected spectra for lensed LBGs. We did not search the SDSS MGS for LBGs for a number of reasons. For one, the magnitude limit of $r<17.77$ is more than two magnitudes brighter than any lensed LBGs known. For another MGS spectra are automatically identified, and those which are unidentified or for which the two identification pipelines disagree are marked for individual attention. Such galaxies would be identified before we would have access to them.

On the other hand, the $u$-band survey reaches down to $u<20.5$ which is comparable to the observed magnitudes of $\mathrm{cB} 58$ and the $8 \mathrm{O}^{\prime}$ Clock Arc. Because they are special plates they do not receive the same attention that the MGS plates do so there is an op- 
portunity to find something missed by others. Lastly, due to the faint limiting magnitude there are a number of unidentified spectra with noisy spectra and no cmission lines.

Unlike the photometric searches detailed above this is an analysis of spectra already in hand requiring completely different methods. The spectra are compared to template spectra of LBGs through a method called cross-correlation. Cross-correlation can potentially find similarities between spectra that are not immediately obvious to the untrained eye even while simultaneously determining the redshift using Fourier techniques. The crosscorrelation technique is set forth by Tonry \& Davis (1979) and is described below.

Before this method can be implemented it is necessary to prepare both the spectra of interest and the template spectra for cross-correlation. These steps are shown for the cB58 spectrum in Figure 5.8. The first step is to rebin the spectra into logarithmic wavelength bins. The logarithmic wavelength bins, $\Delta \ln \lambda$, must be the same size for all templates and spectra. The observed wavelength is related to the rest-frame wavelength by $\lambda=\lambda_{0}(1+z)$. By taking the logarithm of the wavelength the shift in the spectrum due to redshift becomes linear, $\ln \lambda=\ln \lambda_{0}+\ln (1+z)$. This is a vital step when the spectra have an unknown redshift or velocity.

The second step is to remove the continuum. The cross-correlation technique is aimed at matching absorption and emission lines as well as sharp features in the spectrum such as the Lyman break. Removing the continuum prevents the cross-correlation from getting hung up on large scale features which could potentially wash out the information provided by line features. To determine the shape of the continuum the spectrum is median filtered using a wide swath of wavelengths both to the red and blue of each wavelength. 
We constructed the continum using the median of the spectrum over a wavelength range roughly $160 \AA$ wide in the optical centered at each continuum wavelength. The actual width of the median bin changes with wavelength as the spectra are binned logarithmically. The median smoothed continuum is then subtracted from the observed spectrum to obtain the: continuum subtracted spectrum.

Next cosine bell apodization is applied to the continuum-subtracted spectrum. The purpose of cosine bell apodization is to ensure that the spectrum goes smoothly to zero at both ends. Because the spectrum is treated as periodic in the cross-correlation analysis it is important for the ends of the spectrum to match each other. Otherwise there can be a discontinuity where the the two ends of the spectrum meet. Such a sharp feature can fool the cross-correlation creating strong, erroneous peaks in the results. To solve this problem the the continuum-subtracted spectrum is multiplied by a cosine bell function. A cosine bell function is equal to 1 everywhere except on the ends where it goes smoothly to zero like a cosine function. In our apodization the bell function drops from 1 to 0 over roughly $250 \AA$ in the optical on either end of the spectrum.

The last step in the preparation of the spectra and templates is the bandpass filtering of the apodized, continuum-subtracted spectra. Bandpass filtering removes both the high frequency variations due primarily to noise and the low frequency variations missed by the continuum subtraction which could lead to erroneous results. Bandpass filtering is accomplished by first taking the Fouricr transform of a spectrum, then mulliplying the the transformed spectrum by the bandpass filter and lastly taking the inverse Fourier transform 
to return the bandpass filtered spectrum. The bandpass filter, $b(k)$, employed is given by

$$
b(k)=\left\{\begin{array}{ccc}
0 & \text { for } & k<\frac{1}{30} \frac{N}{2 \pi} \\
30 \frac{2 \pi}{N}\left(k-\frac{1}{30} \frac{N}{2 \pi}\right) & \text { for } & \frac{1}{30} \frac{N}{2 \pi} \leq k<\frac{1}{30} \frac{N}{\pi} \\
1 & \text { for } & \frac{1}{30} \frac{N}{\pi} \leq k<\frac{1}{2} \frac{N}{2 \pi} \\
1-\frac{4 \pi}{N}\left(k-\frac{4 \pi}{N}\right) & \text { for } & \frac{1}{2} \frac{N}{2 \pi} \leq k<\frac{N}{2 \pi} \\
0 & \text { for } & k \geq \frac{N}{2 \pi}
\end{array}\right.
$$

At wavenumbers in the middle the transformed spectrum is unaltered. On the edges the transformed spectrum linearly decreases until it reaches 0 on both the high and low wave number extremes.

With the spectra and templates properly prepared cross-correlation can begin. If $f(n)$ is the spectrum of an unknown object at an unknown redshfit as a function of logarithmic wavelength bin $n$ and $t(n)$ is a template spectrum at $z=0$ then the normalized cross-correlation function $c(n)$ is given by

$$
c(n)=f \times t(n)=\frac{1}{N \sigma_{f} \sigma_{t}} \sum_{m=0}^{N-1} f(m) t(m-n)
$$

where $N$ is the number of logarithmic bins and $\sigma_{f}$ and $\sigma_{t}$ are the root mean square of the spectra given by

$$
\sigma_{f}^{2}=\frac{1}{N} \sum_{n} g(n)^{2}
$$

However it is computationally advantageous to work in Fourier space. Equation 5.4 gives the discrete Fourier transform.

$$
G(k)=\sum_{n} g(n) \exp (-2 \pi i n k / N)
$$


Applying equation 5.4 to equation 5.2 yields

$$
C(k)=\frac{1}{N \sigma_{f} \sigma_{t}} G(k) T^{*}(k)
$$

where $T^{*}(k)$ is the complex conjugate of $T(k)$. By eliminating the summation in equation 5.2 equation 5.5 is much faster computationally. The last step is to take the inverse Fourier transform of equation 5.5 to return to $c(n)$.

To function $c(n)$ is interpreted by searching for peaks in the function. The strength of the peaks indicate the degree to which a spectrum matches the template. As $c(n)$ is a normalized function $c=1$ indicates a perfect match to the template. The location of the peak indicates the redshift of the object. By counting the number of wavelength bins the peak value of $c$ is offset the observed wavelength can be found by multiplying by $\Delta \ln \lambda$.

Four template spectra were used in the search. They are the three coadded LBG spectra in Figure 4.1 from Shapley, et al. (2003) and the cB58 spectrum in Figure 4.2 from Pettini, et al. (2000).

Figure 5.9 shows the results from the cross-correlation of the cB58 spectrum to the four LBG template spectra. In the top panel of Figure 5.9 the cB58 spectrum is cross-correlated to itself. This leads to a sharp peak with a perfect $c=1$ centered on $n=0$ indicating no redshift difference between the spectra. The main peak in the top panel clearly dominates all other maxima. In the second panel the cB58 spectra is crosscorrelated with the coadded spectrum of the weakest quartile of Ly $\alpha \mathrm{EW}$ in Figure 4.1. In this case the peak value of $c$ is only $\sim 0.3$ but it is still stronger than any other peak. It too is centered very near $n=0$. This corresponds to a redhsift of $z=0.002$ meaning. that the templates are at essentially the same redshift. As mentioned in $\S 1.7$ absorption 
features are often shifted by internal dynamics so a small difference in redshift between the templates is not surprising. Comparing the two spectra in Figures 4.1 and 4.2 there are several differences, most notably perhaps the fact that cB58 has much stronger absorption features. Nonetheless the cross-correlation technique is successful in finding the similarities between the two spectra and clearly determining the correct redshift.

In the bottom two panels of Figure 5.9 the $\mathrm{cB} 58$ spectrum is cross-correlated with the coadded spectra of the middle two quartiles of Lya emission. Here the cross-correlation fails to find the similarities between the spectra. No peak in $c$ dominates. If one were to assume the strongest peak is the correct one the measured redshift would be $z \sim 0.7$ for both templates. In both cases the correct peak can be seen near $n=0$ however it is not distinguished from they many other peaks. Part of the reason behind this is that the absorption features which match the cB58 spectrum become weaker in the coadded spectra as the Ly $\alpha \mathrm{EW}$ increases. The other is that the Ly $\alpha$ emission line is so much stronger than any other feature in the spectrum that it becomes the only relevant feature in the crosscorrelation. As the cB58 spectrum has no significant emission lines the cross-correlation becomes hung up on many erroneous peaks and fails.

Searching the $u$-band survey spectra using this technique yielded no results. The technique was also applied to the spectra from the $2 \mathrm{dF}$ data runs at the AAT. Given the large number of weakly exposed objects it was thought that cross-correlation may be able to pick out some objects that were missed by eyc. IIowever this also proved unsuccessful. 


\subsubsection{SDSS J113658.36+024220.1}

There have been a number of objects found in the SDSS data by members of the collaboration which were initially believed to by strongly lensed or very luminous LBGs. With the exception of the 8 O'Clock Arc these objects have all proven to be something else. Perhaps the most interesting of these objects is SDSS J113658.36+024220.1, the spectrum of which is shown in Figure 5.10.

One project goal of the SDSS is to catalog the diversity of quasars in the universe. Due to the very large size of the SDSS very rare objects are observed. For example Schneider, et al. (2003) provide a catalog of 16,713 quasars in SDSS Data Release 1 and Hall, et al. (2002) describe 23 broad absorption line quasars with different, unusual properties. SDSS J1136+0242 was discovered as part of these efforts. SDSS J1136+0242 was automatically targeted for follow-up spectroscopy for two reasons. The first is that it was identified as a quasar candidate. Secondly it was identified as a serendipity target. The serendipity category is designed to allow explore regions of parameter space outside of the norm to find unusual objects (Stoughton, et al. 2002). SDSS J1136+0242 is identified as a serendipitous target because its position is coincident with an object with more than $1 \mathrm{mJy}$ in the FIRST catalog. FIRST (Faint Images of the Radio Sky at Twenty $\mathrm{cm}$ ) is a radio survey of the North Galactic Cap at 1365 and $1435 \mathrm{MHz}$ using the NRAO Very Large Array (Becker, White, \& Helfand 1995).

In late 2002 it was noticed upon visual inspection that the spectrum of SDSS J1136+0242 had several strange properties which are inconsistent with AGN activity. Chief among these is the fact that the spectrum (Figure 5.10) has strong Ly $\alpha$ emission while C IV, 
Si IV, and C II are in absorption. The absence of C IV emission is particularly vexing. In addition there is a dip in the spectrum at $1280 \AA$ ( $4470 \AA$ in the observed franne) which is not usually seen in quasars but is common in LBGs (Shapley, et al. (2003), see Figure 4.1).

At this point its discovery was announced within the collaboration. Immediately the LBG target list generated by the first generation algorithm was checked to see if SDSS J1136+0242 was included, and if not why. The object was not on the target list. The most obvious reason why it was not on the list is because it is unresolved in the SDSS data and classified as a star. As described earlier in each selection algorithm many steps were taken to avoid contamination by stellar sources. Despite efforts to the contrary a significant fraction of the objects observed in the summer of 2001 had stellar spectra. Given the number of stars masquerading as extended objects and surviving the star rejection method of the time there was no plausible way to include point sources for consideration. Strongly lensed LBGs are expected to be extended objects in the SDSS and, with the benefit of hindsight, cB58 and the $8 O^{\prime}$ Clock Arc are extended. SDSS J1136+0242 would not be rejected as a star under the first generation criteria but would be rejected on the basis that it is not in the orange LBG selection area of Figure 4.8 .

However in investigating SDSS J1136+0242 with respect to the first generation algorithm an interesting discovery was made. At the time it was not possible to query the survey with a database interface. To find an object of interest at a known position one had to consult the lists of observing runs to detcrmine which one covered the area in question. From there within the runs the correct camera column and field number had to be found. Then the photometry information could be found by searching the relevant tsObj catalog 
Table 5.2. Photometry of SDSS J113658.36+024220.1

\begin{tabular}{ccccccc}
\hline MJD & Date & $u \pm \sigma_{u}$ & $g \pm \sigma_{g}$ & $r \pm \sigma_{r}$ & $i \pm \sigma_{i}$ & $z \pm \sigma_{z}$ \\
\hline \multirow{2}{*}{51668} & May 4, 2000 & $22.44 \pm 0.37$ & $20.69 \pm 0.04$ & $20.06 \pm 0.03$ & $19.61 \pm 0.03$ & $19.28 \pm 0.08$ \\
51901 & Dec 23, 2000 & $21.92 \pm 0.17$ & $20.54 \pm 0.03$ & $19.97 \pm 0.02$ & $19.60 \pm 0.03$ & $19.12 \pm 0.07$ \\
51989 & Mar 21, 2001 & - & $20.50 \pm 0.17$ & $19.86 \pm 0.13$ & $10.45 \pm 0.11$ & - \\
\hline
\end{tabular}

file.

By design the SDSS imaging camera has six columns of five CCDs. Observations are done in drift scanning mode where the telescope points in a fixed direction and as the Earth turns the celestial objects drift down the column of five CCDs, one each for the five SDSS ugriz filters. In one pass each object is observed in all five filters. The six CCD columns are not directly adjacent to each other- there is a gap between the columns. In order to achieve full coverage of the sky a second set of observations must be made slightly offset from the first such that the gaps in the first set are observed. Each set is referred to as a strip and when put together they form a stripe which has no gaps. The two strips have a small amount of overlap so that parts of the sky are unobserved and to facilitate photometric calibrations between the strips. In all around $10 \%$ of objects in the SDSS have been observed twice.

By pure random chance SDSS J1136+0242 falls in the overlap between two strips. It was first observed on May 4, 2000 and the second observation was made seven months later on December 23, 2000. The photometric data can be found in the first two rows in Table 5.2. It is immediately noticeable upon first glance that SDSS J1136+0242 appeared 
to have brightened over the seven months between observations. The increase in brightness was dependent on color and in the $i$ band it did not change at all.

The fact that SDSS J1136+0242 experienced a significant change in brightness strongly points to the fact that it is not an LBG. The high luminosities of LBGs are powered by young, massive stars. These stars have lifetimes on the order of a million years so significant changes in the brightness of star forming galaxies should vary on similar time. scales, not half a year. Another issue is causality (see Krolik (1999)). If all points on an object with diameter $l$ changes brightness at all points simultaneously the light from the far edge will be observed with a delay $l / c$ compared to the light from the front edge. For this reason the increase or decrease in brightness will have a width of $l / c$. This gives the shortest. time scale on which an object can fluctuate in brightness across the full volume. For a galaxy the size of the Milky Way which is of order $10^{5}$ light years across this time scale is 100,000 years. AGN on the other hand are powered by accretion onto disks surrounding supermassive black holes at the centers of galaxies. These accretion disks have sizes comparable to the size of the Solar System- much smaller than the size of galaxies. AGN are known to fluctuate in the optical on timescales as short as weeks.

Hall, et al. (2004) find that the two photometric observations vary at the $99.8 \%$ confidence level. At the redshift of SDSS J1136+0242 $(z=2.492)$ the seven month gap between observations corresponds to a delay of 67 days in the rest-frame. In addition, they were able to synthesize gri magnitudes from SDSS spectroscopic measurenents on March 21, 2001. These measurements are shown in the last row of Table 5.2. The synthetic magnitudes vary at the $85 \%$ level from the first photometric observations but do not vary 
from the second.

Hall, et al. (2004) conclude that SDSS J1136+0242 is not a strongly lensed LBG for three reasons. First is the photometric variability previously discussed. Secondly the fact that it is unresolved in the SDSS suggests that it is not a lensed galaxy. Furthermore imaging from the Canada-France-Hawaii Telescope, a $3.6 \mathrm{~m}$ telescope atop Mauna Kea, yields a FWHM of 0.59 " which is equivalent to the stars in the field. Gravitational lensing images with separations larger than 0.2 " are ruled out. In addition the SDSS and CFHT images show no objects within 11" which constrains the parameters of the potential lensing object. If a galaxy cluster is responsible for the lensing it would have to have a redshift $z \geq 1$ to elude detection in the imaging. The imaging data does not rule out lensing but makes it unlikely. Lastly, the tilt in the rest-frame UV spectrum is redder than for typical LBGs and no spectral synthesis models for young stellar populations reveal a continuum as featureless as that observed in SDSS J1136+0242.

\subsubsection{SDSS Southern Stripe Image Co-Add}

The SDSS Southern Stripe image co-add is a data product currently undergoing testing within the SDSS collaboration and is not publicly available at this time. These data are from the South Galactic Cap imaging survey (York, et al. 2000). During the fall only the South Galactic Cap is observable from APO. Figure 4.10 which shows the distribution of many known galaxy clusters also shows where the plane of the Milky Way cuts across the sky and blocks the light from external galaxies. The SDSS only observes above and below the plane of the galactic plane due to its primary design goal of measuring the large scale structure of galaxy clustering: Nearly the entire North Galactic Cap is observable from 
APO. The aim of the SDSS is contiguous coverage of the bulk of the North Galactic Cap in imaging and spectroscopy. However for the Southern Galactic Cap there is a roughly equal amount of time to cover a much smaller area. In the Southern Galactic Cap only three stripes are observed. These stripes stretch from $310^{\circ}$ to $60^{\circ}$. One of these follows the celestial equator. The other two are on great circles, one above and one below the equatorial stripe. These stripes are observed repeatedly for two reasons. For one it makes it possible to find variable objects including supernovae in external galaxies. Secondly it allows the images to be co-added to reach about two magnitudes fainter than the imaging survey in the north.

Catalog added data have been available for some time. Catalog added data for an object can found when an object has been identified and measured by the photometric pipeline in multiple observations. As previously described luptitudes preserve all flux information by design, even when the flux is negative. For each observation of an object the photometry within an aperture can be converted without loss to fux and an error weighted average can be taken across many observations. The mean fluxes can then be converted back into fluxes. For faint objects near the survey limits this technique can reduce the Poisson errors in the photometry resulting in more precise photometry. The amount of the improvement varies across the Southern Stripe depending on how many observations are available for a given area.

While adding the catalog data can reduce the crrors in weakly detected objects it does not reduce the limiting magnitude of the survey. In order for the flux of an object to be stored in a catalog it must be bright enough to be detected in the first place. For the 
catalog added data this means an object must be detected in each individual image which all have exposures of the same length. In order to go deeper the individual images must be summed into a new image with a longer effective exposure time. The summed image is then run through the photometric pipeline. The image co-added data not only improves the $\mathrm{S} / \mathrm{N}$ of objects found in individual exposures but it also finds many objects that are only revealed with longer exposures.

One of the major problems in creating the image co-added catalog is that the number of observations for each objects. For one thing if an object straddles the edge of two regions with different numbers of observations it becomes more difficult to robustly measure the area. Another key challenge is building a robust, bias free sample from a catalog of objects with differing exposure times. While this would be a large concern for the project described in chapter 2 it is of minimal consequence here where strongly lensed LBGs are being searched for by any means necessary. Objects with substantially better photometry than the norm only increase the odds of the project's success. If at some point a large sample of lensed LBGs were found then making conclusions about the density of the objects on the sky or their global properties would be made more difficult by variable photometry quality. At this point it is an academic point.

The beta version of the Southern Stripe image co-add covered an area of approximately 30 square degrees. There are on average 25 individual observations being combined for each location. The data are not yet in a database and cannol be accessed by SQL query. Rather the raw tsObj catalog FITS files need to be manipulated directly as was the case when the first generation algorithm was in use. 
IDL code was written to impose the conditions of the SQL database query of the current algorithm. Only one modification was made. The current SQL query requires that, $17.77 \leq i \leq 20.5$ in Petrosian magnitudes. However the co-add data are advertised to be two magnitudes fainter than the standard imaging data. In light of this fact the requirement; was changed such that $i \geq 17.77$. This rejects objects which are automatically observed spectroscopically as part of the MGS while allowing for an independent determination of what the new limiting magnitude should be. Within the 30 square degrees available there are 214,686 objects which meet these requirements.

The next step is to apply the flag filter from the current selection algorithm. This leaves 178,780 objects, or $83 \%$ of those passing the first round of cuts. From there the advanced star separation techniques are used in a manner identical to those in the current selection algorithm. This leaves 132,127 objects or $62 \%$ of the objects surviving the original cut.

No targets were found in the Southern Stripe co-add that were particularly encouraging. One interesting note is that the new color cuts described in section 4.3 .3 returned objects which upon inspection of the images were almost exclusively diffraction spikes and other obviously spurious photometry. This the opposite of what was seen in the standard imaging results where the objects returned were nearly always clearly actual stars or galaxies. The reason for this is unclear.

Dus to poor weather only onc object selected from the cu-added dala was observed. The spectrum obtained is of low quality but the best guess is that it is a $z=4.53$ quasar. It is heartening that this contaminant is a true denizen of the high redshift universe. However 
it is a second puzzling object which passes all of the tests indicating it is an extended source yet has the spectrum of a quasar. 

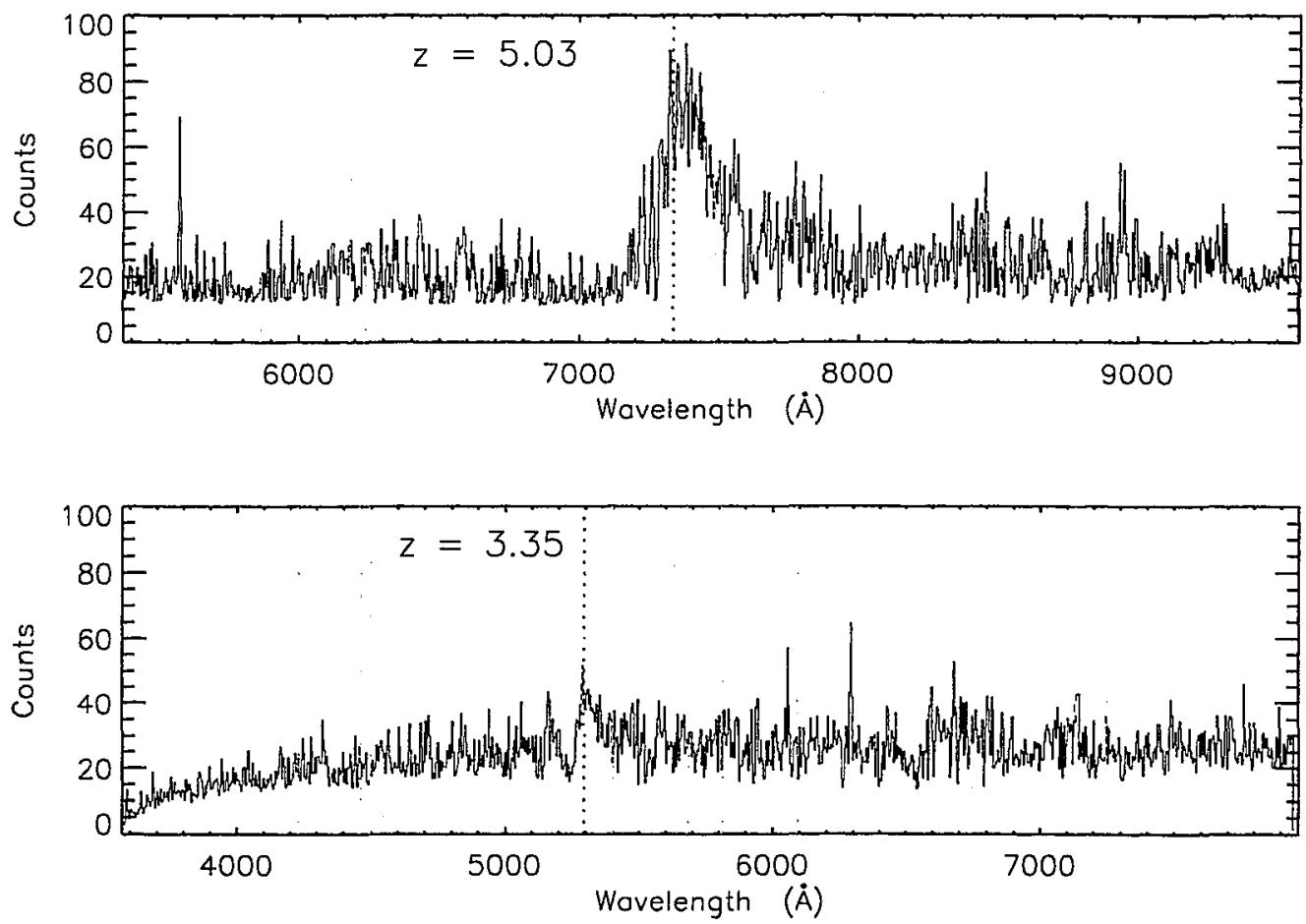

Figure 5.1 High redshfit quasar spectra observed in July 2001. These objects were targeted as strongly lensed LBGs but were revealed to be quasars by their spectra. Both spectra were taken with the $2 \mathrm{dF}$ instrument on the AAT. The top panel shows SDSS J001714.67100055.5 , a quasar at $z=5.03$, observed with the red spectrograph. The bottom panel shows SDSS J145121.82+010811.8, a quasar at $z=3.35$, observed with the blue spectrograph. The spectra are not fux calibrated. The dotted red line indicates the position of Lyc in the redshifted frame. The violet lines are the positions of other prominent lines in the ultraviolet. 


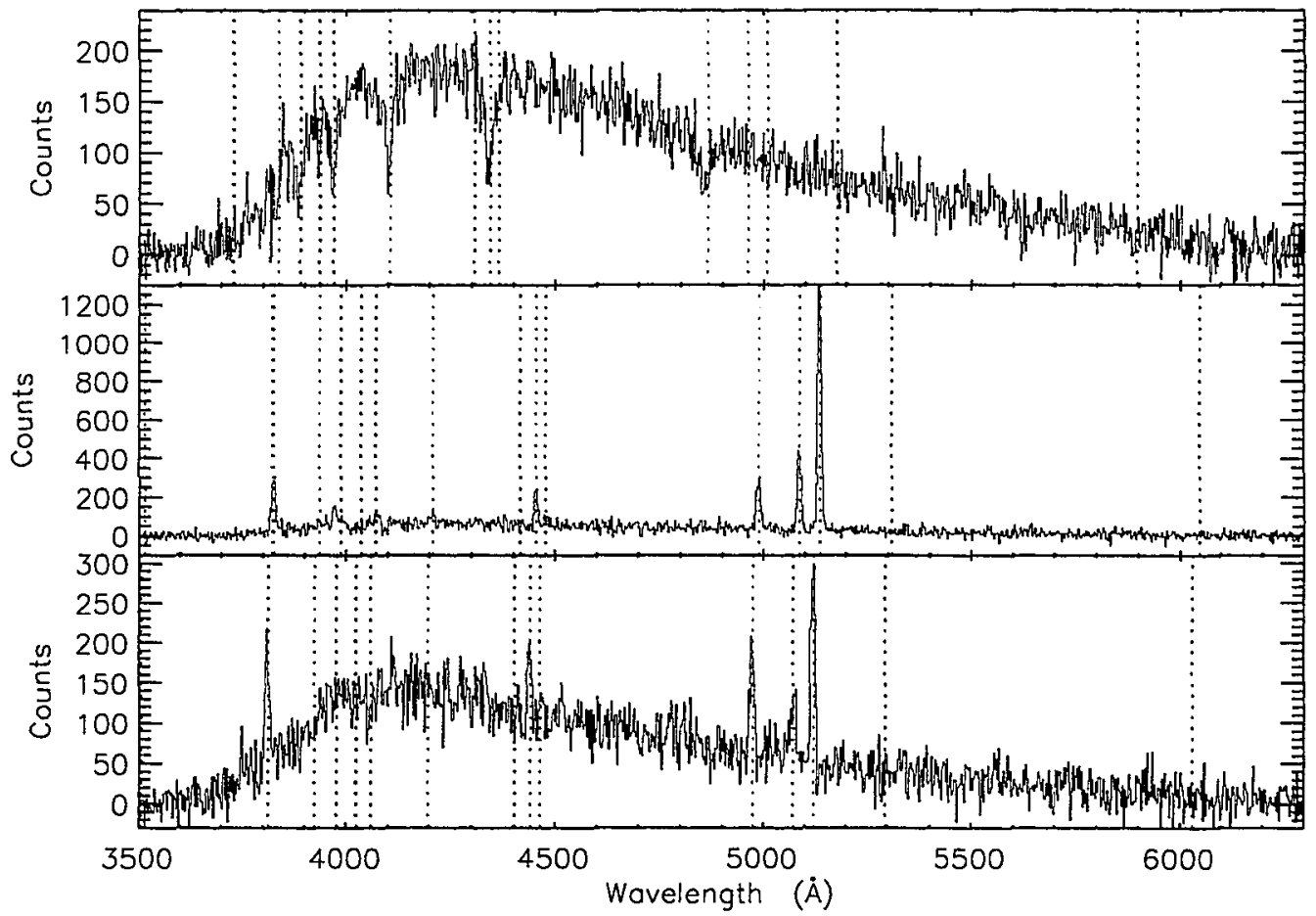

Figure 5.2 Subaru FOCAS spectra of candidate lensed LBGs identified by the second generation selection algorithm. Top panel: SDSS J020655.21-005817.4, an A type star. Middle panel: SDSS J224750.77+000149.7, an emission line galaxy at $z=0.0255$. Bottom panel: SDSS J031314.03-065123.0, an emission line galaxy at $z=0.0226$. The spectra are not flux calibrated. The dotted lines mark the redshifted positions of prominent lines. The magenta lines are Balmer series hydrogen lines, the green lines mark the positions of prominent stellar absorption features, and the cyan lines mark collisionally excited forbidden lines. 

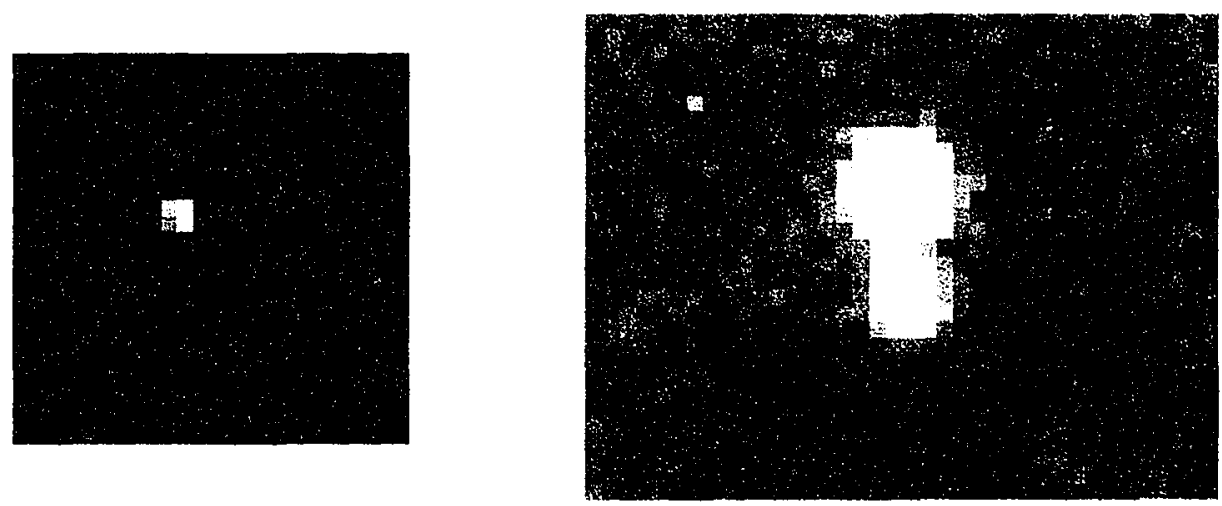

Figure 5.3 Failure of the SDSS imaging star/galaxy classification. At left is the SDSS image of SDSS J020655.21-005817.4 and at right is the image from the slit viewer on the 8.2m Subaru Telescope on Mauma Kea. The object is classified as an extended source in the SDSS, but the superior seeing at Mauna Kea and spatial resolution of the Subaru instrument clearly reveal that it is actually two adjacent point sources. 

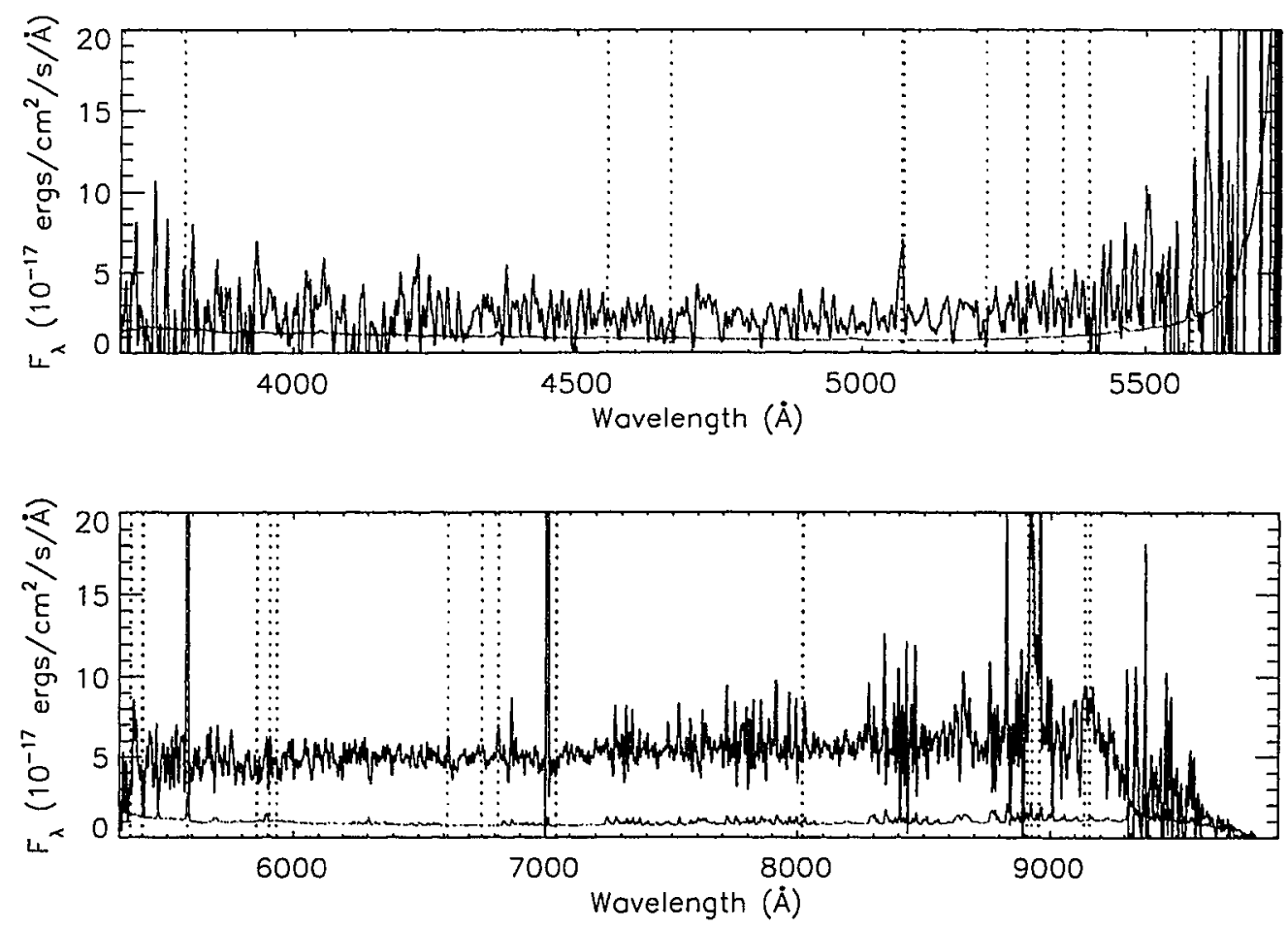

Figure 5.4 DIS III spectrum of candidate lensed LBG SDSS J142617.23+390145.8. Spectroscopy reveals this object is a galaxy with weak emission lines at $z=0.36$. The top panel shows the half of the spectrum from the blue spectrograph and the bottom panel shows the half from the red spectrograph. The red line in both panels gives the noise level. The dotted lines mark the redshifted positions of prominent lines. The magenta lines are Balmer series hydrogen lines, the green lines mark the positions of prominent stellar absorption features, and the cyan lines mark collisionally excited forbidden lines. 

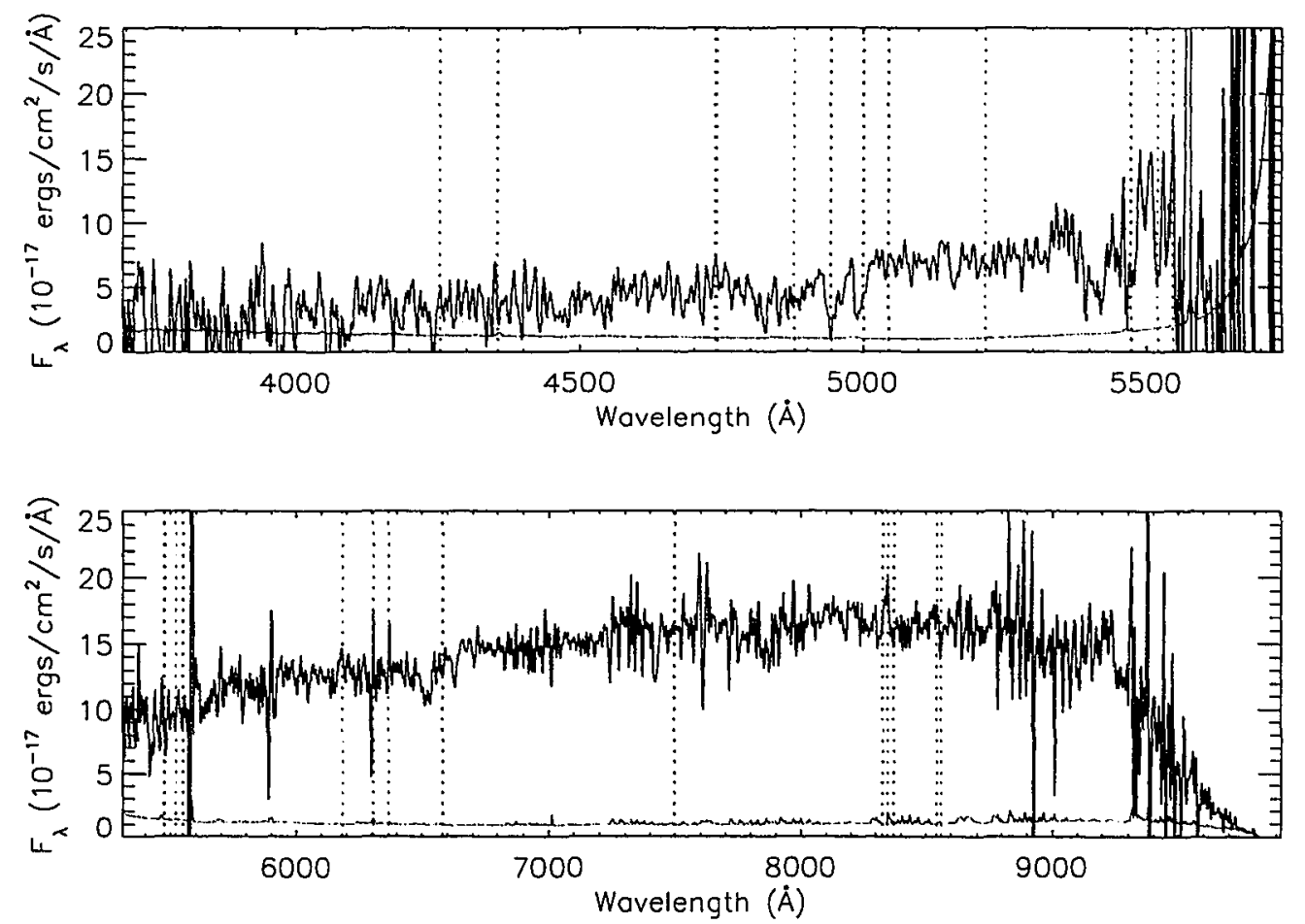

Figure 5.5 DIS III spectrum of candidate lensed LBG SDSS J154718.85+205619.5. Spectroscopy revels this object to be a galaxy at $z=0.264$ with a pronounced Balmer break. The description of the plot follows that of Figure 5.4. 

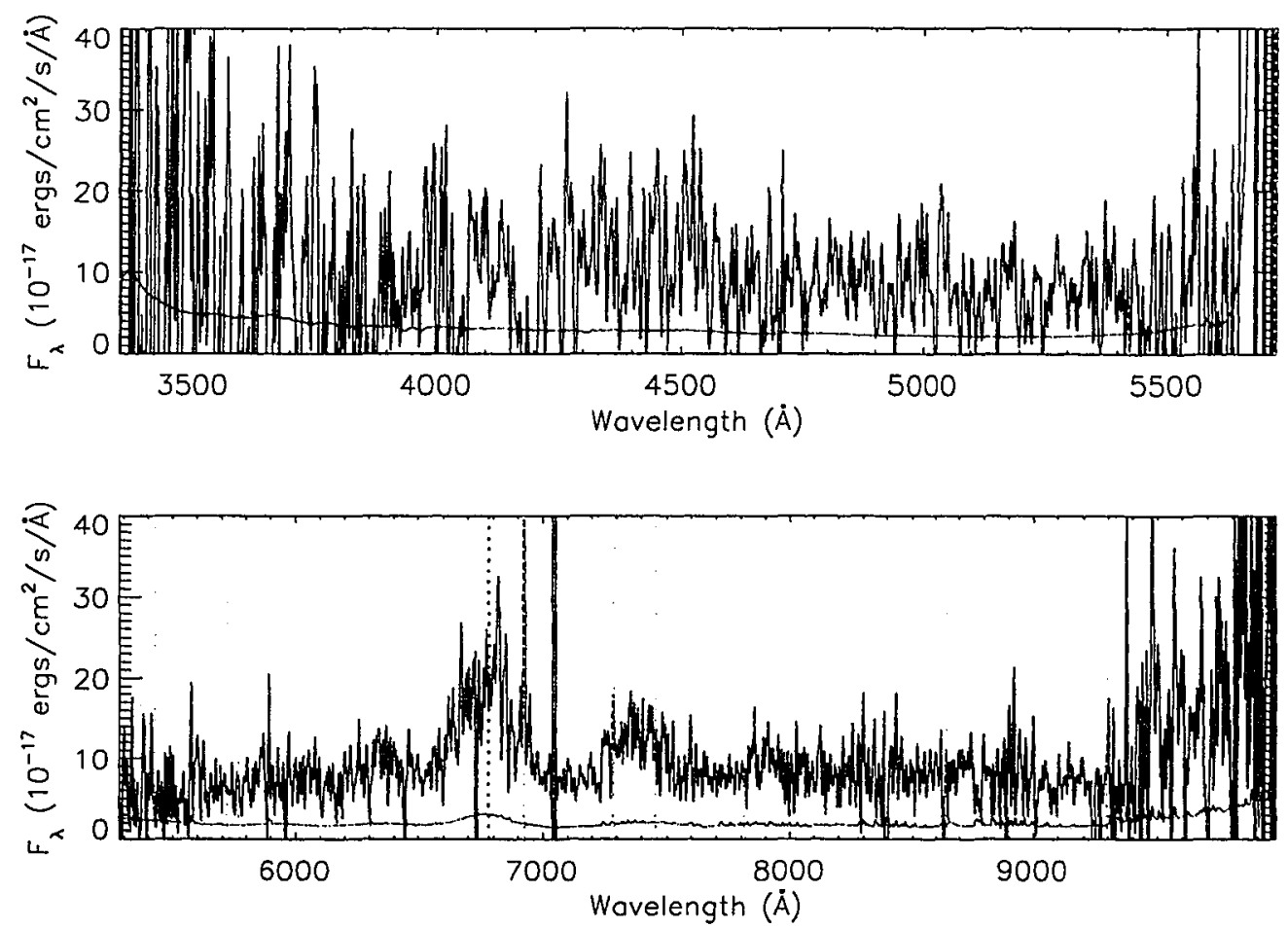

Figure 5.6 DIS III spectrum of candidate lensed LBG SDSS J100051.00+232610.0. Spectroscopy reveals this object is a quasar at $z=4.58$ even though the object is clearly extended. The description of the plot follows that of Figure 5.4. The black dotted line shows the redshifted position of the Ly $\alpha$ line and the violet dotted lines show the redshifted positions of other common lines in the ultraviolet. 


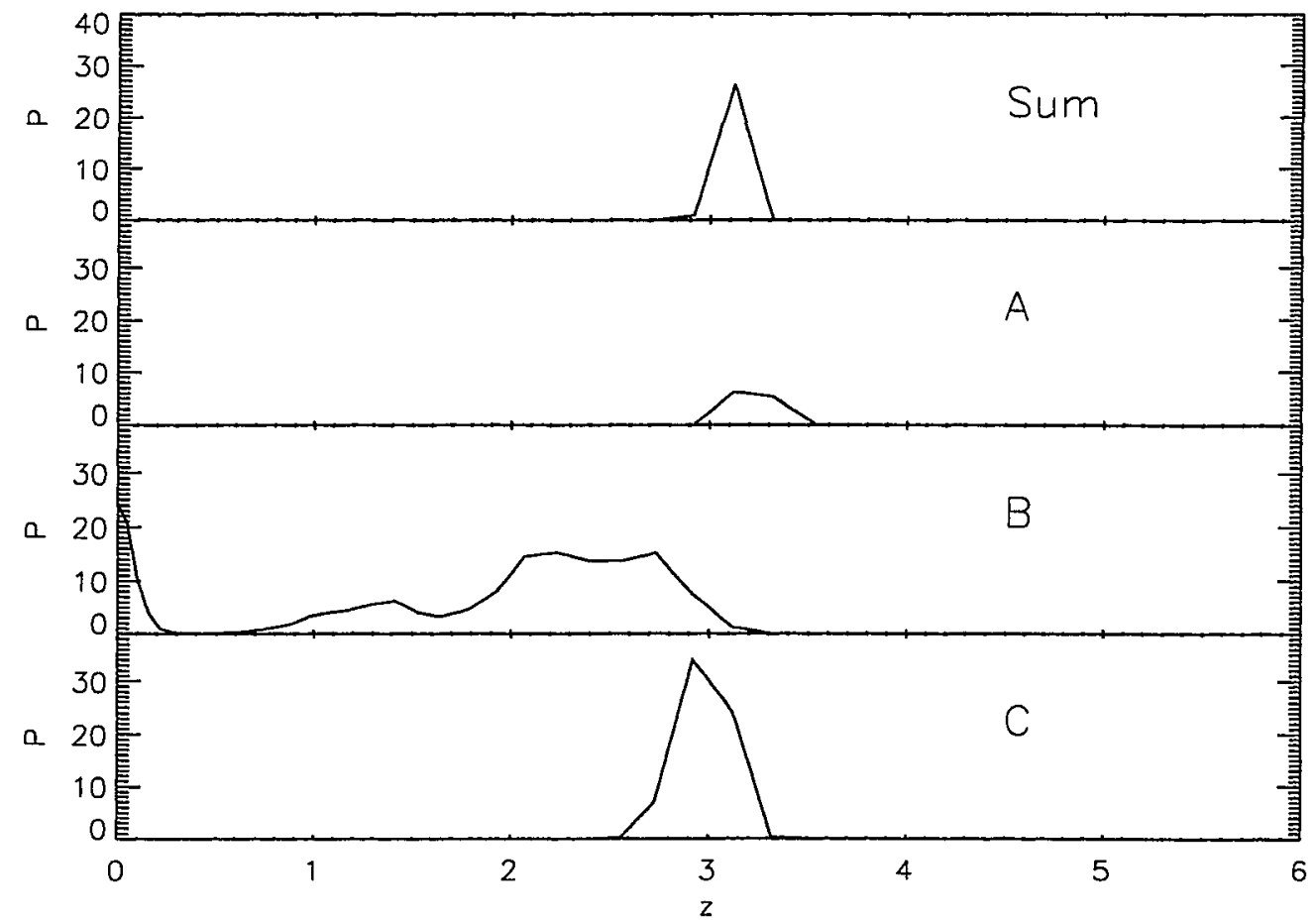

Figure 5.7 Photometric redshifts for the 8 O'Clock Arc and parts thereof. The probability an object is at a given redshift is given for the the whole $8 \mathrm{O}^{\prime}$ Clock Arc (top panel) and the pieces $A, B$, and $C$ (second, third, and bottom panels respectively) which make up the full arc. 


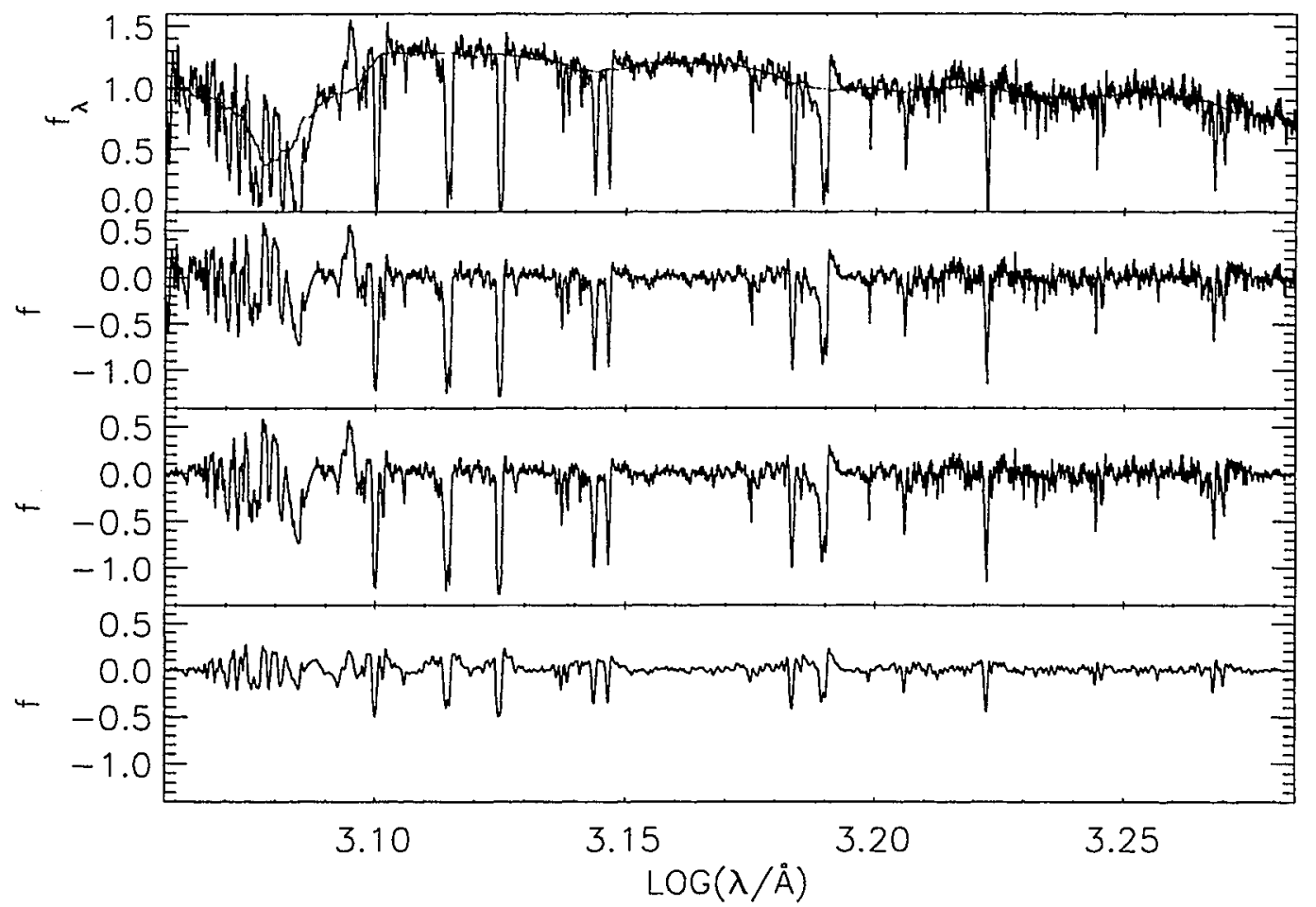

Figure 5.8 Steps taken to prepare spectra for cross-correlation. Top panel: The cB58 spectrum from Figure 4.2 converted to $f_{\lambda}$ and rebinned logarithmically. The red line is the continuum fit following the description in the text. Second panel: The same spectrum following continuum subtraction. Third panel: The continuum subtracted spectrum after cosine bell apodization. Bottom panel: Final spectrum used for cross-correlation. This is the result of bandpass filtering the spectrum in the third panel. 


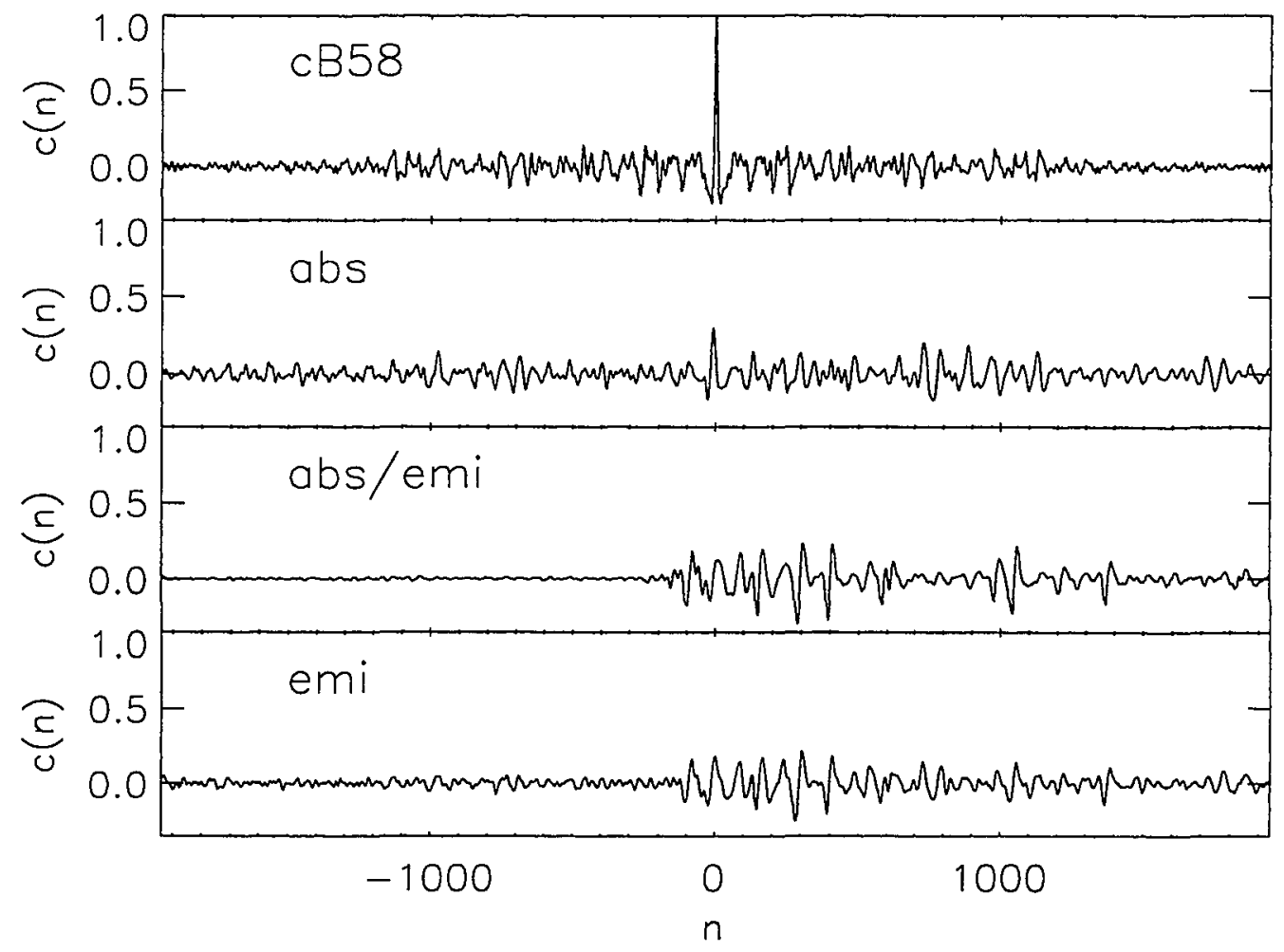

Figure 5.9 Cross-correlation of the cB58 spectrum with the four LBG template spectra. The plots show the value of the function $c(n)$ from equation 5.2 for each bin number $n$. The top panel shows the cross correlation of the $\mathrm{cB} 58$ spectrum with itself, a perfect match with a peak $c$ value equal to 1 . The bottom three panels show the correlation with the spectra from Figure 4.1 ranging from the quartile weakest in Lyo emission in the sccond pancl to the second strongest at bottom. 


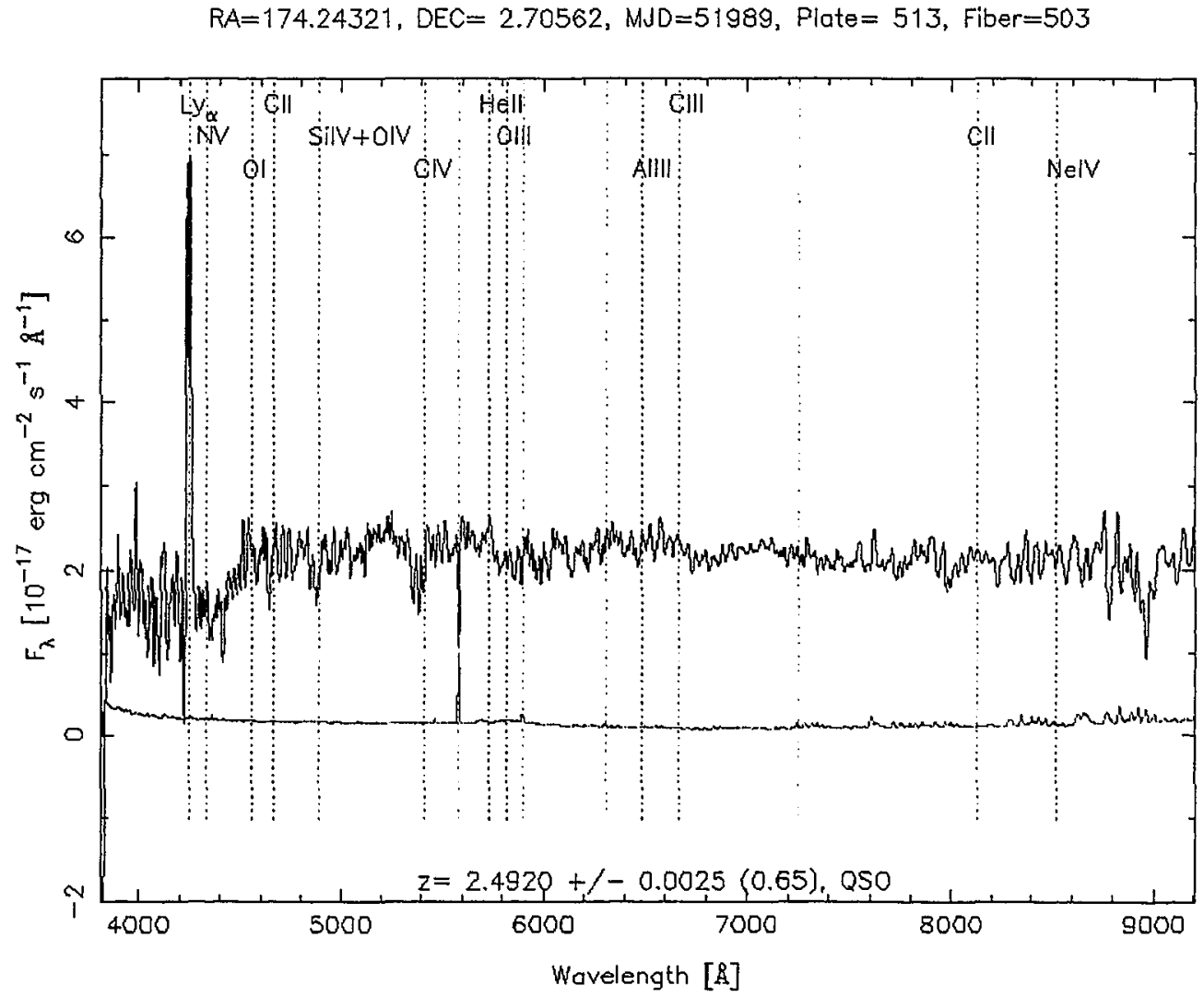

Figure 5.10 SDSS spectrum of the Lyman $\alpha$-only quasar SDSS J113658.36+024220.1. Vertical dotted lines mark the positions of selected absorption and emission lines at the measured redshift of $z=2.4920$. The green line shows the error in the spectrum as a function of wavelength. 


\section{Chapter 6}

\section{Extremely Metal Poor Galaxies}

This chapter discusses the serendipitous discovery of a candidate extremely metal poor galaxy (XMPG) and a subsequent effort to systematically select them from the SDSS. As discussed in Chapter 4 the first incarnation of lensed LBG search opportunistically piggy backed onto the Chiu (2006) study of the epoch of peak quasar activity. Quasar activity reached its maximum at $z \sim 3$. Despite the fact that quasars were the most active at this point in time the quasars of that time are observationally difficult to study because their colors become confused with those of stars on the stellar locus. To combat this problem Chiu (2006) used a shotgun approach to take spectra of as many objects in this region of color space as possible. More than a decade ago this approach would have been prohibitively time consuming. However with the advent of the multi-fiber spectrograph it has become a reality.

Observations were made with the $2 \mathrm{dF}$ instrument on the $3.9 \mathrm{~m}$ Anglo Australian Telescope in Coonabarabran, New South Wales, Australia. The 2dF instrument can simultaneously take spectra of 400 objects within a two degree field of view (Lewis, et al. 2002). This greatly reduces the amount of time to acquire spectra. The region of color space 
targeted by Chiu (2006) is dominated by stars. For each pointing with $2 \mathrm{dF}$ only around ten objects proved to be quasars. The remainder, minus a few broken fibers and 15-20 sky fibers, were stars or indiscernible.

There were however a few surprises. Figure 6.1 shows the spectrum of SDSS J094725.23-000509.3. The spectrum was taken with the Double Beam Spectrograph on the 2.3 Advanced Technology Telescope at Siding Spring Observatory outside Coonabarabran, New South Wales, Australia. The top panel of the figure shows that the object has very strong emission lines on top of a weak continuum. The bottom panel provides a closer view of the continuum and weak emission lines. The first odd thing is that the spectrum of SDSS J0947-0005 reveals that it is an emission line galaxy at redshift $z=0.0568$. Galaxies are not expected to be contaminants because the targeting algorithm of Chiu (2006) selects only unresolved sources as it is designed to find quasars.

The particularly interesting feature of SDSS J0947-0005 can be more clearly seen in Figure 6.2. Figure 6.5 is a close-up of Figure 6.1 emphasizing the wavelength region around the $\mathrm{H} \alpha$ line. The dotted magenta line denotes the position of the $\mathrm{H} \alpha$ line at $z=0.0568$. The cyan lines show the positions of redshifted metal lines. The [S II] doublet (with rest-frame wavelengths of $6718 \AA$ and $6733 \AA$ ) lies at $7100 \AA$ and [N II] lines (at $6550 \AA$ and $6585 \AA$ in the rest-frame) bracket. The [S II] doublet is barely detected and the [N II] lines appear to be missing. The fact that these metal lines are so poorly detected while the hydrogen lines are detected with high $\mathrm{S} / \mathrm{N}$ is a strong indication that SDSS J0017-0005 lias a pautlicularly low metallicity. For actively star forming galaxies in the SDSS the median value of the flux ratio of $\mathrm{H} \alpha$ to [N II] at $6584 \AA$ is 2.9. For SDSS J0947-0005 it is $150 \pm 26$. 
The image of SDSS J0947-0005 is shown in the upper right of Figure 6.3. It is immediately obvious that it is quite compact and easy to understand how it could be classified as a point source. For reference the red object to the upper right of SDSS J09470005 is classified as a point source while the white object to the lower right is determined to be an extended source, although the difference between the two appears to be subtle. The color appears more purple than I Zw 18 in the upper left of Figure 6.3. This is due to the difference in redshift. SDSS J0947-0005 is more distant at $z=0.0568$ compared to $z=0.0025$ for $\mathrm{I} \mathrm{ZW} 18$. The redshift also helps account for the difference in angular size. Although I Zw 18 is clearly extended in the SDSS it has high surface brightness near the center and does not appear to extend very far from the bright regions. As such it is considered compact.

These factors taken together make it a strong candidate to be a extremely metal poor blue compact dwarf (BCD) galaxy. Given this discovery the possibility that other such galaxies had gone undetected was immediately considered. For the quasar study of Chiu (2006) the two details of interest are whether an object is a quasar, and if so what is its redshift. The 400 spectra from each $2 \mathrm{dF}$ pointing are reduced together using an automated reduction package unique to the instrument. The spectra are then inspected visually to look for the broad emission lines in the rest-frame ultraviolet of quasars at $z \sim 3$. Generally a galaxy with strong, narrow optical emission lines is quickly identified as a low redshift object and ignored. Therefore it was quite possible that other similar galaxies were missed.

A new pass through the spectra revealed a second object, SDSS J154311.30+011235.4. The image of SDSS J1543+0112 can be found in the lower left of Figure 6.3. The image 
reveals that its appearance is nearly identical to that of SDSS J0947-0005. It too is compact and unresolved in the SDSS photometry. It also has a nearly identical color. The spectrum of SDSS J1543+0112 is shown in Figure 6.4. The spectrum was taken with the Double Imaging Spectrograph II instrument on the ARC 3.5m telescope at Apache Point Observatory in New Mexico. The spectrum is also very similar to Figure 6.1. It features a very weak continuum with strong emission lines. It has a redshift of $z=0.0621$ which is also very similar to SDSS J0947-0005. The close up of the H $\alpha$ line in Figure 6.5. This reveals a weak [S II] doublet and weaker [N II] lines. The $\mathrm{H} \alpha$ to [N II] ratio for SDSS J1543+0112 is $350 \pm 200$

Following this discovery several challenges remained. The first was to try and find similar objects that may have been overlooked in the SDSS spectroscopy. Secondly accurate measurements of the metallicity were needed to identify the candidates as XMPGs. Lastly the old stellar populations of the XMPGs needed to be constrained in order to determine if they are truly experiencing their first bursts of star formation. The remainder of this chapter describes the selection method used to target these galaxies, metallicity measurement techniques and results, and near infrared photometry taken to constrain the old stellar populations of the true XMPGs.

\subsection{Target Selection}

The target selection for the XMPGs comes directly from Chiu (2006). The target selection was designed to quickly cut down the number of objects in the sample due to data storage constraints. The first step was to reject all objects with declinations $\delta>10^{\circ}$. 
This is because observations were performed using the AAT. At the southern latitudes of Australia objects with high declinations cannot be observed. Secondly it was required that. all objects be classified as point sources in the SDSS photometry and that

$$
0.6<u-g<1.5
$$

Like in the algorithms discussed in Chapter 4 it the SDSS photometry flags are used to reject data which are potentially problematic. Objects with one or more of the BRIGHT, EDGE, CR, SATURATED, and CHILD flags set were rejected.

A hard magnitude limit was not set, but rather priority was given to observing the brightest objects. This depended on the density of objects in the field. In a sparse field objects as faint as $i=22.0$ could be targeted.

The SDSS pipeline targets point sources which follow equation 6.1 and $0.0<$ $g-r<0.2$ as quasars. These objects are targeted for spectroscopy down to $i=20.2$. Chiu (2006) adjusts the $g-r$ cutoff value such that the number of targets matches the number of fibers available. There is no lower bound on $g-r$. The upper value in $g-r$ is not a fixed value, but rather a curved line tangent to the stellar locus which is moved up and down. By using a curved line the number of stellar contaminants added by increasing the upper bound on $g-r$ is reduced relative to a straight line.

In total 149.1 square degrees were surveyed. The number of spectra inspected was $\sim 24,500$. Most of these objects were stars within the Galaxy. There were 340 quasars discovered at $z \sim 3$ and 5 at $z \sim 5$. In addition the two previously described candidate XMPGs, SDSS J0947-0005 and SDSS J1543+0112, were found.

In an attempt to cast a bigger net the SDSS spectra were searched for galaxies 
with similar properties to the ones found in $2 \mathrm{dF}$. Objects with similar $\mathrm{H} \alpha$ and [O III] line ratios as the $2 \mathrm{dF}$ objects were identified and pulled out.

Unfortunately the objects found with SDSS spectra do not, match the quality of SDSS J0947-0005 and SDSS J1543+0112. Figure 6.6 shows one of these objects, SDSS J023958.57-003149.1. While the strength of the emission lines of this object are similar to those of the XMPG candidates in Figures 6.1 and 6.4 the continuum is clearly detected and much stronger. This is readily apparent in the bottom panel of Figure 6.6. A closer inspection of the $\mathrm{H} \alpha$ line in Figure 6.7 shows a clear detection of the [S II] doublet and $[\mathrm{N} \mathrm{II}]$ is also observed. The ratio of $\mathrm{H} \alpha$ to $[\mathrm{N} \mathrm{II}]$ is much lower as well at $11.5 \pm 1.3$. In addition the SDSS image at the bottom right of FIgure 6.3 reveals an object which is clearly extended. It also appears diffuse, perhaps with some spiral structure. This is a departure from I $\mathrm{Zw} 18$ at upper left which has high surface brightness at the center with a sharp drop off in surface brightness at the edge. The strength of the metal lines in Figure 6.7 suggest that while SDSS J0239-0031 likely has low metallicity it probably does not qualify as an XMPG.

Two of the 25 objects were previously identified. SDSS J122622.70-011513.9 and SDSS J125526.06-021334.1 both appear in the Kniazev, et al. (2004) catalog of strong emission line H II galaxies in SDSS DR1. Their selection method consisted of identifying objects with large Balmer line equivalent widths and rejecting AGN and LINERS. The last step was to measure oxygen abundances and reject objects for which the uncertainty in the abundance measurements was larger than $0.2 \mathrm{dex}$. The oxygen abundances of these objects were measured to be 7.92 and 7.83 respectively. 
A better feel for the nature and metallicity of these objects can be obtained with a BPT diagram (Baldwin, Phillips, \& Terlevich 1981). Figure 6.8 shows a BPT diagram for the candidate XMPGs discussed here. The BPT diagram is a plot of the logarithms of the $[\mathrm{N} \mathrm{m}] / \mathrm{H} \alpha$ and $[\mathrm{O} \mathrm{III}] / \mathrm{H} \beta$ emission line flux ratios. From a practical standpoint these flux ratios are useful in that since in both ratios the wavelengths of the two lines are close to each other and thus less affected by reddening. The $[\mathrm{O} \mathrm{III}] / \mathrm{H} \beta$ ratio is sensitive to the temperature and ionization level in a nebular region. The $[\mathrm{N} \mathrm{II}] / \mathrm{H} \alpha$ is sensitive to the Nitrogen abundance. The red line, from Kauffmann, et al. (2003), divides AGN from normal star forming galaxies. Objects below and to the left of the line are star forming galaxies. Objects above and to the right are AGN. LINERS fall to the right of the line below the AGN and planetary nebula are above the line towards the left of the diagram. AGN tend to have high ionization levels and nitrogen abundances which place them in the upper right of the diagram.

The candidate XMPGs targeted from the SDSS spectroscopy are plotted as green circles. The candidate XMPGs from the 2dF quasar survey, SDSS J0947-0005 and SDSS $\mathrm{J} 1543+0112$, are plotted as magenta triangles. The location of $\mathrm{I} \mathrm{Zw} 18$, as determined from SDSS spectroscopy, is denoted by the blue diamond. All of the points fall below the red line indicating that their emission lines are indeed powered by star formation. This plot also show that the bulk of the targets from the SDSS spectroscopy fall on the contours of the galactic locus. This indicatos that thcy arc not particularly special and do not have: notably low metallicities.

On the other hand I $\mathrm{Zw} 18$, the two candidates from the $2 \mathrm{dF}$ quasar survey, SDSS 
J0947-0005 and SDSS J1543+0112, and one from the SDSS spectroscopy SDSS J1255-(0213 have low $[\mathrm{N} \mathrm{II]} / \mathrm{H} \alpha$ ratios which clearly distinguish them from the bulk of the objects in the SDSS. However none of the targets matches the low ratio of I $\mathrm{Zw} 18$ which suggests that, they do not break the low metallicity record of $\mathrm{I} \mathrm{Zw}$ 18. The one outlier object with both low $[\mathrm{N} \mathrm{II}] / \mathrm{H} \alpha x$ and $[\mathrm{O} \mathrm{III}] / \mathrm{H} \beta$ ratios has a large uncertainty in the $[\mathrm{N} \mathrm{II]} / \mathrm{H} \alpha$ ratio which indicates that it is not particularly interesting.

In order to classify the candidates as XMPGs more precise metallicity measurements need to be employed. The next section describes these techniques and the results from applying them.

\subsection{Metallicity Measurement Techniques}

The metal contents of a galaxies are most frequently measured in one of two different general methods. For one stellar absorption lines can be measured from galaxy spectra. Through analysis of several line ratios the metallicity can be inferred. Because these lines come from the photospheres of stars the metallicity found using this method is that of the stellar population. The second method is to measure galaxy emission lines and infer the metallicity from emission line ratios. Galaxy emission lines are generated primarily in gaseous nebular regions. As such emission line metallicity measurements probe the metallicity of the gas in galaxies, and more specifically the gas in star forming regions and near AGN.

The stellar metallicity and the gas phase metallicity are not necessarily the same and frequently differ. The expectation is that the gas phase metallicity should be higher 
than the metallicity in stars. The metallicity of a star is dependent on the metallicity of the gas that it formed from at some point in the past. As a result the metallicity measured from the stellar population is an average of the gas phase metallicity at different epochs. The gas phase metallicity on the other hand is a reflection of the metal content of the gas today. Due to stellar evolution each generation of stars should contribute metals to the interstellar gas in a galaxy through supernovae. These metals build up in the interstellar medium over time. Therefore at any point the gas phase metallicity should be higher than the metallicity that has been frozen into the past generations of stars. This is not a steadfast rule. If a galaxy accretes pristine or low metallicity gas from an external source the gas phase metallicity may be lower than that in the stellar population.

The method used to measure the metallicity is largely determined by the nature of the object being observed. Old, red, elliptical galaxies with minimal present day star formation lack emission lines so the gas phase metallicity cannot by measured using emission lines. However these galaxies often have pronounced stellar absorption lines which can be used to measure the stellar metallicity. On the other hand in emission line galaxies with weak continua, as seen in Figures 6.1 and 6.4, the stellar absorption features cannot be measured with enough precision to determine stellar metallicities very well. If the emission lines are well detected the gas phase metallicity can be found.

Other methods for measuring metallicities are briefly described in Kunth \& Östlin (2000). In resolved stellar populations the stellar abundances can be measured photometrically. The color magnitude diagram of a stellar population provides insights on the metallicity of the stellar population. The color of the red giant branch (RGB) is sensitive 
to the metallicity of stars older than 1 Gyr. Similarly the width in color of the RGB is sensitive to the level of variation in the metallicity in a stellar population.

There are a number of other parameters of stellar populations which are sensitive to the metallicity but cannot be definitively converted to metallicities due to secondary effects which are not well understood. These include the color and shape of the horizontal branch in the color magnitude diagram. From a spectroscopic standpoint the fraction of carbon stars among late type giants and the ratio of carbon rich to nitrogen rich Wolf-Rayet stars are also related to the metallicity. Unfortunately the techniques used to determine the metallicity of resolved stellar populations is restricted to the Local Group due to observational constraints.

The galaxies in this sample all have strong emission lines so emission line techniques are used here to measure the gas phase metallicity. The following sections describe diagnostics of emission lines and the results for the candidate galaxies.

\subsubsection{Five-Level Approximation Methods}

Osterbrock (1989) discusses the calculation of the emission line spectra of gaseous nebulae. Gaseous nebulae include AGN, planetary nebulae, and the star forming galaxies of interest here. In order for nebulae to radiate emission lines a source is required which emits photons energetic enough to ionize hydrogen. These sources are accretion disks in the case of AGN, newly formed white dwarf stars in the case of planetary nebulae, and massive, young $\mathrm{O}$ and $\mathrm{B}$ stars in star forming regions.

The general idea is that ultraviolet photons with energies at or greater than 13.6 $\mathrm{eV}$ are absorbed by neutral hydrogen in the nebula. This ionizes the hydrogen and any excess energy is imparted upon the released electron. These electrons collide with other 
free electrons and ions to create a thermal distribution of velocity with a temperature which is generally between 5,000 and 20,000 K. Collisions between electrons and ions result in low-level excitation of the ions. Downward transitions from many of these levels are not likely as they violate electric dipole selection rules. These are traditionally referred to as forbidden lines. This is something of a misnomer as these transitions are not impossible. The forbidden transitions occur radiatively with time scales on the order of seconds. For comparison the time scale is $\sim 10^{8} \mathrm{~s}$ for allowed transitions. For normal gas densities on Earth, and even at the lowest pressures in laboratories, an ion will experience numerous collisions within a second and will be collisionally de-excited. From a practical perspective these transitions do not happen on Earth and are effectively forbidden. At the extremely low densities found in nebulae the collision time scale begins to exceed that of the forbidden transitions. At this point forbidden transitions occur before they can be collisionally deexcited. The energy radiated in these lines escapes the clouds due to the extremely low absorption cross sections of these transitions and the relative paucity of metal ions in the clouds. The other main source of emission lines is the recombination spectra of hydrogen and helium from the recapture of free elections.

Analysis of gaseous nebulae consists of determining the level of ionization, density, and cooling rate for each region of the nebula. The ionization level is dictated by the balance between ionization and recapture of electrons. The highest ionization states are found in the center near the source of high cncrgy photons. Regions of equal iunizalion level are usually spherical shells around the central source. In thermal equilibrium the energy put into the nebula by photoionization is balanced by energy lost to recombination, free-free 
emission and radiative line cooling. The cooling rate is directly related to the strength of the collisionally excited emission lines.

Five-level approximation methods are useful for determining the metal abundances of astrophysical objects with measurable emission lines. The crux of the five-level approximation is the fact that most of the ions which usually dominate nebular cooling have ground-state electron configurations of either $p^{2}, p^{3}$, or $p^{4}$. All three of these electron configurations have five low lying levels which can be collisionally excited by electrons with energies of a few $\mathrm{eV}$. The assumption is that only these five levels are relevant for calculating the observed spectrum as higher levels are not significantly populated by collisions, recombination, or other effects. For many ions transitions between these five levels span wavelengths from the ultraviolet into the infrared.

Several publicly available codes exist which implement the five-level approximation. De Robertis, Dufour, \& Hunt (1987) provide a code for determining electron temperatures and densities given emission line ratios and line emissivities given the temperature and density. The IRAF NEBULAR package (Shaw \& Dufour 1995) uses this program at its core and improves the functionality by adding several other programs to automate the full analysis of the abundances of multiple metals in a nebula. The IRAF NEBULAR package is used below for the abundance analysis of the XMPG candidates.

The first step in determining nebular abundances is to find the electron temporaturc and donsity. The IR $\Lambda F$ lask temden is used for this purpose. These values are determined from line ratios. Some line ratios are particularly sensitive to either the electron density or temperature. Lists of these ratios can be found in Shaw \& Dufour 
(1995). For instance, the electron temperature can be determined from [O III] lines using $I(4959+5007) / I(4363)$, where $I(4959+5007)$ is the sum of the intensities of the [O III] lines at $4959 \AA$ and $5007 \AA$. An example of a line ratio which is sensitive to the electron density is the $I(6716) / I(6731)$ of $[\mathrm{S} \mathrm{II}]$.

Both of these ratios contain emission lines from a single ion. In the case of the [O III] ratio the $4363 \AA$ line is due to a transition from the upper ${ }^{1} S_{0}$ level to the middle ${ }^{1} D_{2}$ and the $4959 \AA$ and $5007 \AA$ lines are transitions from ${ }^{1} D_{2}$ down to lower level ${ }^{3} P$ states. These levels differ significantly in energy so their relative populations are sensitive to the temperature. Because the excited levels are collisionally excited by free electrons the highest, level will be increasingly populated with higher electron temperatures.

For [S II] the situation is somewhat different. Both the $6716 \AA$ and $6731 \AA$ lines are downward transitions from the ${ }^{2} D$ levels to the ${ }^{4} S_{3 / 2}$ ground state. These lines have nearly the same excitation energy. As a result the relative excitation rates of the two levels depends only on the ratio of the collision strengths.

Neither the temperature nor density is constant throughout the whole nebula. As the distance from the central source of ionizing photons decreases the level of ionization and temperature decrease. The density may increase. To handle this problem the NEBULAR package contains the zones code which, given the fluxes of many emission lines, determines the electron temperatures and densities in three zones. The zones are split into low, medium, and high ionization states. For instance the temperature derived from [O III], a medium ionization state, should not be used to analyze [O II] emission lines, a low ionization state. The $[\mathrm{O} I \mathrm{II}]$ and $[\mathrm{O} \mathrm{III}]$ ions are for the most part located in different regions of the nebula. 
The abund task takes the output of the zones task and uses the solutions for the three zones to determine the abundances of all ions for which there is enough information.

In theory this is a straightforward, albeit involved, calculation and full solution for the properties of each zone and the abundances for all atomic species can be found. However this calculation can be limited by the nature of the data available. The wavelengths of the collisionally excited emission lines range from the ultraviolet into the infrared. For a full solution spectroscopy of the whole wavelength range is necessary. While optical spectra can be observed fairly easily the ultraviolet lines must be measured form space. In addition lines in the near-infrared such as [S III] at $9069 \AA$ and $9532 \AA$ can be difficult to observe amid atmospheric $\mathrm{OH}$ lines. In the interest of a full solution there are other important cooling lines in the five line approximation that are not covered in the NEBULAR package. These include the ${ }^{3} P_{1}$ to ${ }^{3} P_{0}$ and ${ }^{3} P_{2}$ to ${ }^{3} P_{1}$ transitions of [O III] which are 88.4 and 51.8 microns in the far infrared. These lines also can only be observed from space. This study is limited to optical spectra available for the candidate XMPGs so only a partial solution is possible.

Another limitation is the fact that the density indicators all involve emission lines with similar energies. The separations of these pairs is typically a few angstroms. To separate these lines and measure them individually requires high resolution spectroscopy. Even then if the lines are significantly broadened by physical processes in the nebula separating them may not be possible. The [S II] doublet is an exception to this problem with a separation of $15 \AA$ but it still poscs a problem when ahempling to measure the electron densilies in other ionization zones.

The last challenge arises from the physical nature of the candidate XMPGs. Many 
of the emission lines of interest for abundance analysis are intrinsically weak and difficult, to observe. This is compounded by the fact that the XMPG candidates are dwarf galaxies with redshifts of $z \sim 0.05$ which makes them faint themselves and more difficult to observe than local H II regions. Lastly the candidate XMPGs have low metallicities which weakens many of these lines of interest even further. Many of the lines used by zones and abund are simply not detected.

In the following sections the results of the abundance determination from the fivelevel approximation for each candidate XMPG are discussed.

\section{Results}

The spectrum used for the abundance measurements of SDSS J0947-0005 was taken with the Double Beam Spectrograph. Given the limitations discussed above the abund task is able to measure electron density for the low ionization zone from [S II] and electron temperatures for the low ionization zone from [O II] and for the medium ionization zone from [O III] for SDSS J0947-0005. In the low ionization zone the density found was $7,900 \mathrm{~cm}^{-3}$ and the temperature was $7,900 \mathrm{~K}$. In the medium ionization zone the temperature was 17,300 K. In the absence of measured densities and temperatures the software assumes a default value of $1000 \mathrm{~cm}^{-3}$ and temperature of $10,000 \mathrm{~K}$. Within these limitations abundances were determined for the neutral oxygen and the $\mathrm{O}^{+}$and $\mathrm{O}^{++}$ions. The $\mathrm{O}^{+}$and $\mathrm{O}^{++}$ions had similar abundances with an neutral oxygen abundance about 40 times lower than each of the ion species. Together this is an oxygen abundance of $12+\log (\mathrm{O} / \mathrm{H})=7.95$. The limit for upper limit for oxygen abundances in XMPGs is 7.65 so this object does not appear to be an XMPG. 
Abundances of a few other ions can be determined from the spectrum of SDSS J0947-0005. For neon the abundance of $\mathrm{Ne}^{++}$is $12+\log \left(\mathrm{Ne}^{++} / \mathrm{H}\right)=6.88$. The abundances for sulfur are 5.71 for $\mathrm{S}^{+}$and 5.30 for $\mathrm{S}^{++}$for an total sulfur abundance of 5.85 . For $\mathrm{N}^{+}$ the abundance is 5.89 .

For SDSS J1543+0112 things are more complicated. The spectrum used for abundance analysis was taken with DIS II at APO. The $I(6716) / I(6731)$ of [S II] has a value of 2.3 which is outside of the allowed range. This is not particularly disturbing as the ratio is within $2 \sigma$ of the maximum allowed value. To compensate for this the maximum allowed ratio is assumed which, according to Osterbrock (1989), corresponds to electron densities anywhere from 10 to $100 \mathrm{~cm}^{-3}$. The higher value is assumed for the low ionization zone to keep it in closer agreement to the $1000 \mathrm{~cm}^{-3}$ assumed for the medium ionization zone. The temperatures measured for SDSS J1543+0112 are 12,900 $\mathrm{K}$ in the low ionization zone and $18,000 \mathrm{~K}$ in the medium ionization zone.

The resulting abundances for SDSS J1543+0112 are $12+\log (\mathrm{O} / \mathrm{H})=7.71,5.74$ for sulfur, 6.54 for $\mathrm{Ne}^{++}$, and 5.10 for $\mathrm{N}^{+}$. Again the oxygen abundance is too high for SDSS $J 1543+0112$ to be considered an XMPG, although it is lower.

The last XMPG candidate is SDSS J1255-0213, for which only the SDSS spectrum was available. It has an [S II] $I(6716) / I(6731)$ ratio of 1.5 which is just above the maximum allowed value of 1.45 . Again a density of $100 \mathrm{~cm}^{-3}$ is assumed for the low ionization region and $1000 \mathrm{~cm}^{-3}$ for the medium ionization region. The temperature is determined to be 1,600 $\mathrm{K}$ in the low ionization zone and $16,200 \mathrm{~K}$ in the medium ionization zone. For SDSS J12550213 the abund task fails to find abundances for neutral oxygen and $\mathrm{O}^{+}$. A lower limit for 
the oxygen abundance is given by $12+\log \left(\mathrm{O}^{++} / \mathrm{H}\right)=7.74$. The nitrogen abundance is 6.80 . Again SDSS J1255-0213 has too high of an oxygen abundance to qualify as an XMPG.

\subsubsection{Line Ratio Methods}

The five-level approximation is a powerful tool for determining the abundances and physical conditions of nebular regions. However as the previous section demonstrates it can be difficult to implement. A good deal of telescope time, both ground and space based, needs to be dedicated to each object to achieve the $\mathrm{S} / \mathrm{N}$ and resolution necessary for the full analysis. In many cases this is not possible or practical either because the objects are intrinsically too faint or there are too many objects in a sample. Very few objects in the high redshift universe can be analyzed in this way. Even so metallicity is such an important property that it is impossible to get a full picture of the physics of an object without it.

In response to this problem a lot of energy has been put into to efforts to estimate the metal content of galaxies from more easily observable properties of galaxies. These efforts are usually centered on emission line flux ratios of the most easily observable lines. The most common of these in the optical are hydrogen Balmer series, [O III], and [N II] lines. These methods are calibrated using galaxies where oxygen abundances have been measured by the more robust five-level methods.

One of the line ratios sensitive to the oxygen abundance is the ratio of the $[\mathrm{N} \mathrm{II}]$

line at $6584 \AA$ to the $\mathrm{H} \alpha$ line at, $6565 \AA$. This ratio is quite insensitive to reddening because the lines are so close together in wavelength. On the other hand this can result in them being blended if the spectral resolution is too low or if the velocity dispersion is too large. StorchiBergmann, Calzetti, \& Kinney (1994) show a linear relationship between the [N II]/Hc ratio 
Table 6.1. Measured oxygen abundances of candidate XMPGs

\begin{tabular}{lccc}
\hline \multirow{2}{*}{ Name } & \multicolumn{3}{c}{$12+\log (\mathrm{O} / \mathrm{H})$} \\
& NEBULAR & {$[\mathrm{N} \mathrm{II}] / \mathrm{H} \alpha$} & O3N2 \\
\hline & & & \\
SDSS J0947-0005 & 7.95 & $7.62 \pm 0.08$ & $7.78 \pm 0.02$ \\
SDSS J1543+0112 & 7.71 & $7.09 \pm 0.70$ & $7.67 \pm 0.12$ \\
\hline SDSS J1255-0213 & $\geq 7.74$ & $7.75 \pm 0.04$ & $7.82 \pm 0.02$ \\
\hline I Zw 18 & $7.13 \pm 0.03$ & $6.87 \pm 0.10$ & $7.78 \pm 0.02$ \\
\hline
\end{tabular}

and the oxygen abundance $12+\log (\mathrm{O} / \mathrm{H})$ for star forming galaxies with abundances greater than 8.5. Pettini \& Pagel (2004) extend this relationship down to oxygen abundances of 7.0. They also find a linear relationship, but they find that a third-order polynomial is a slightly better fit.

The left half of Figure 6.9 shows a histogram of the oxygen abundances of the candidate XMPGs as calculated from the $[\mathrm{N} \mathrm{II}] / \mathrm{H} \alpha$ ratio using the cubic function from Pettini \& Pagel (2004). The red line indicates the value of I $\mathrm{Zw} 18$ as determined from the SDSS spectroscopy. The figure reveals that most of the objects have oxygen abundances below the solar value but for the most part are not anomalously low. By this standard there are three XMPGs in the sample. These are the three objects which were discussed more in depth in the previous section. Table 6.1 lists the oxygen abundances calculated from each method considered for the three top candidates and I Zw 18.

Table 6.1 shows that the oxygen abundances measured by the $[\mathrm{N}$ II] $/ \mathrm{H} \alpha$ ratio are in varying levels agreement with those derived from the more in depth analysis using 
the NEBULAR package. For SDSS J1255-0213 the abundance is in perfect agreement with the lower limit derived using the NEBULAR package. In the case of SDSS J1543+0112 an extremely low metallicity is found. However the error is so large that it is in agreement with the five-level value and is not a convincing XMPG. For both SDSS J0947-0005 and I $\mathrm{Zw} 18$ the metallicites determined form the $[\mathrm{N} \mathrm{II}] / \mathrm{H} \alpha$ ratio are significantly lower than the five-level values. I $\mathrm{Zw} 18$ has a particularly low metallicity which is outside of range for which the Pettini \& Pagel (2004) equation is valid. Their linear relation would yield an abundance of $7.39 \pm 0.03$ which is too high. Either way the discrepancy is likely due to poor calibration. The same camnot be said for the discrepancy in SDSS J0947-0005 which is well within the calibrated region.

The O3N2 ratio was introduced Alloin, Collin-Souffrin, \& Joly (1979). The O3N2 ratio is defined by Pettini \& Pagel $(2004)$ as $\mathrm{O} 3 \mathrm{~N} 2 \equiv \log \{([\mathrm{O}$ III $] \lambda 5007 / \mathrm{H} \beta) /([\mathrm{N}$ II $]$ $\lambda 6583 / \mathrm{H} \alpha)\}$. The original definition include the sum of the $4960 \AA$ and $5007 \AA$ lines of [O III] in the first ratio. A linear relationship between O3N2 and oxygen abundance is given by Pettini \& Pagel (2004). The relationship is only recommended for abundances above 8.1, but the relationship continues to lower metallicities although with much larger scatter. The right half of Figure 6.9 shows the oxygen abundances of the candidate XMPGs as determined from the O3N2 ratio. The basic trend is the same: most candidates are in the normal range of abundances and only a few stand out. In general the O3N2 method returns slightly higher abundances and does not yield low abunclinces at all. In Table 0.1 the abundances determined from O3N2 for the top candidates are shown. Despite being outside of the reliable range the results are in good agreement with other methods form 
SDSS J1255-0213 and SDSS J1543+0112. The O3N2 method also lowballs the abundance of SDSS J0947-0005. It does not handle the extremely low metallicity of I Zw 18 at all.

The most widely used abundance indicator is the $R_{23}$ index (Pagel, et al. 1979). The index is defined as $R_{23} \equiv([\mathrm{O}$ II $\lambda 3727+[\mathrm{O}$ III $] \lambda \lambda 4959,5007) / \mathrm{H} \beta$. As discussed in Kewley \& Dopita (2002), the $R_{23}$ index is double valued with oxygen abundance and is particularly sensitive to the ionization parameter at low metallicities. While it is a useful tool at high metallicities it becomes dubious at oxygen abundances below solar which is where the value of the index peaks. The $[\mathrm{N}$ II] $/ \mathrm{H} \alpha$ can be used to determine whether an object is on the low or high metallicity side of the peak, but even so the relationship between $R_{23}$ and oxygen abundance flattens at sub-solar abundances making the index less useful for low metallicity galaxies and is not used here.

\subsection{Near Infrared Photometry}

As discussed in $\S 1.8$ it would be of great interest to find galaxies which are undergoing their first episode of star formation. This would provide a close up view to study the the early lives of the progenitors of today's galaxies. The first step in identifying these objects, should they exist, is to determine that they have extremely low metallities. This process has been described in the previous section. The second step is to make sure that the current burst of star formation is the first. This is accomplished by constraining the old stellar population.

At ultraviolet wavelengths the luminosity of a galaxy, provided it is forming stars, is dominated by massive, luminous, young, hot stars with lifetimes from a few to tens of 
million years. For this reason the ultraviolet luminosity is a good indicator of the current star formation rate. However these stars provide little flux in the near infrared. At these wavelengths the luminosity of a galaxy is dominated by the old stellar population. As such it is more representative of the total mass of the stars formed in the galaxy over its history.

In order to investigate the old stellar populations of the candidate XMPGs near infrared photometry was taken for many of them. Images were taken in two bands, the $H$ band at $1.6 \mu \mathrm{m}$ and the $K_{S}$ band at $2.2 \mu \mathrm{m}$. The $K_{S}$ band differs from the $K$ band in that it is slightly narrower. This avoids some atmospheric $\mathrm{OH}$ lines which reduces $\mathrm{S} / \mathrm{N}$ and allows longer exposures to be taken before the image is saturated by the sky. The sky is considerably brighter in the near infrared than the optical due in part to $\mathrm{OH}$ lines and also to the blackbody spectrum of the Earth given its temperature. As a result sky saturation is a much larger concern which prohibits exposures of lengths which are common place in optical photometry.

The images were taken on the night of October 30, 2004 using the $3.5 \mathrm{~m}$ ARC Telescope at Apache Point Observatory in New Mexico. The Near Infrared Grism Spectrometer and Imager II (GRIM II) was used for the observations. These were the last science images taken with GRIM II before decomissioning. GRIM II used a 256 by 256 NICMOS3 HgCdTe array with sensitivity from 1 to $2.5 \mu \mathrm{m}$. Observations were hampered by the fact that, one of the four quadrants of the array was not functioning so standard dithering patterns could not be uscd.

The observing sequence involved imaging a standard star in both the $H$ and $K_{S}$ bands, then two science targets in both bands, and so on. This ensured that a standard 
star was observed just before or just after every science observation. This is because the sky is far less stable in the near infrared than in the optical and more susceptible to time variations. In addition the standard stars were picked to be as close as possible to the science targets on the sky. This was done to minimize the effects of spatial sky variations on the photometry.

As mentioned earlier only very short exposures can be made in the near infrared from the ground. For the bright standard stars the exposures are only $2 \mathrm{~s}$ in either band. The object exposures were $5 \mathrm{~s}$ in the $K_{S}$ band and $10 \mathrm{~s}$ in $H$. Deep exposures require multiple exposures. The GRIM II software is set up to handle this automatically. To avoid pixel to pixel variations on the chip dithering was required. The dithering pattern used five different positions. The built in patterns could not be used due to the defective quadrant so a custom pattern was specified. The number of images taken per position was six for the standard stars, six for objects in the $H$ band, and 12 for objects in the $K_{S}$ band. Due to the shutter speed and electronics the true exposure time is 0.2 less per frame than advertised for GRIM II. Combining the above considerations the exposure times were $54 \mathrm{~s}$ for standard stars in both frames, and for science objects they were $294 \mathrm{~s}$ in the $H$ band and $288 \mathrm{~s}$ in the $K_{S}$ band.

Due to right ascension constraints only one of the three best XMPG candiclates was observed. The $H$ and $K_{S}$ band images of SDSS J0947-0005 are shown in Figure 6.10. For reference the SDSS mosaic image is shown in the upper right of Figure 6.3. The location of SDSS J0947-0005 is indicated by the green circle in each panel. SDSS J0947-0005 is not detected in the $H$ band. It is detected in the $K_{S}$ band with a magnitude of $19.68 \pm 0.43$. For 
reference the galaxy in the lower right of each panel is measured to have $H=20.32 \pm 0.28$ and $K_{S}=19.56 \pm 0.38$

The fact that SDSS J0947-0005 is detected in the $K_{S}$ band proves that this object is not experiencing its first episode of star formation. The infrared light implies that a old stellar population already exists. The size of this population can be estimated. Rettura, et al. (2006) use spectral synthesis modeling to measure the stellar masses from the spectral energy distributions of a sample of early-type galaxies. They show that the derived stellar masses exhibit a linear relationship with the $H$ band absolute magnitude, $M_{H}$. Such relationships should be used with caution as they are not a substitute for modeling the full SED. Even then models are degenerate in IMF, age, metallicity, and SFR (which is discussed at greater length in Chapter 2) so exact masses for individual galaxies should be taken with a grain of salt as well. However the goal here is to merely find an estimate of the upper limit of the stellar mass of SDSS J0947-0005. As long as the results are not being overly read into the use of these relationships is appropriate.

SDSS J0947-0005 is not detected in the $H$ band. However it must be true that $H<20.32$ because the galaxy in the lower right corner is detected at this magnitude. Because the redshift has been spectroscopically measured to be $z=0.0568$ the luminosity distance of SDSS J0947-0005 can be calculated assuming the cosmological parameters from the Wilkinson Anisotropy Probe (Spergel, et al. 2003). This in turn yields a distance modulus which lcads to $M_{H} \geq-16.64$ for SDSS J0947-0005. Using tho Rettura, ct al. (2006) relationship the maximum stellar mass in SDSS J0947-0005 is $3 \times 10^{8} M_{\odot}$.

Bell, et al. (2003) estimate the stellar mass to light ratios of the local universe in 
the SDSS ugriz bands and the $K_{S}$ band using data from the 2MASS survey. They find that. in the $K_{S}$ band that $(M / L)_{\odot}=0.95$ with $\sim 30 \%$ error. Using the above distance modulus $M_{K_{S}}=-17.28$. For the $\operatorname{Sun} M_{K_{S}}=3.32$. This leads to a stellar mass of $1.7 \pm 0.5 \times 10^{8} M_{\odot}$ which is in agreement with the upper limit provided by the non-detection in the $H$ band.

To put this in context the current star formation rate of SDSS J0947-0005 can be measured from the observed $\mathrm{H} \alpha$ flux. Kennicutt (1983) developed a conversion to star formation rate from models. The main assumption in this model is the IMF. Using this relationship the current star formation rate is measured to be $0.76 \mathrm{M} \odot \mathrm{yr}^{-1}$. Star formation rates and luminosities for the other XMPG candidates are shown in Table 6.2. At the present rate of star formation it would take only $2 \times 10^{8}$ years to form the stellar mass determined from the $K_{S}$ imaging. Given the weak continuum of SDSS J0947-0005 is unlikely that there has been much star formation activity in the Gyr prior to the current episode. However the detection in the $K_{S}$ band strongly suggests an earlier age of star formation followed by a lull of more than billion years before the present activity. If the previous star formation occurred at the same rate as is presently observed it must have also been a burst lasting at most $200 \mathrm{Myr}$.

Based on the photometry of Hunt, Thuan, \& Izotov (2003) and the SDSS redshift the absolute magnitudes of the NW component of I Zw 18 are $M_{H}=-13.8$ and $M_{K_{S}}=$ -14.0. This shows that I Zw 18 is around 20 times fainter in the near infrared than SDSS J0947-0005 while only 8 times fainter in the $r$ band. Munt, Thuan, \& Izotov (2003) provide an in depth analysis of the maximum age of $\mathrm{I} Z \mathrm{w} 18$ from the near infrared imaging. They conclude that the oldest major stellar population in I Zw 18 is at most $500 \mathrm{Myr}$ old, but 
Table 6.2. Measured parameters of candidate XMPGs

\begin{tabular}{lccccc}
\hline Name & $z$ & $M_{r}$ & $\begin{array}{c}\mathrm{H} \alpha \\
\left(\mathrm{erg} \mathrm{s}^{-1} \mathrm{~cm}^{-2}\right)\end{array}$ & $\begin{array}{c}\text { SFR } \\
\left(M_{\odot} \mathrm{yr}^{-1}\right)\end{array}$ & {$[\mathrm{N} \mathrm{II]} / \mathrm{H} \alpha$} \\
\hline SDSS J0947-0005 & 0.0568 & -15.82 & $1170 \times 10^{-17}$ & 0.76 & $150 \pm 26$ \\
SDSS J1543+0112 & 0.0621 & & & & $350 \pm 200$ \\
\hline SDSS J1255-0213 & 0.0519 & & & & $110 \pm 10$ \\
\hline I Zw 18 & 0.0025 & -13.59 & $1244 \times 10^{-17}$ & 0.0014 & $450 \pm 50$ \\
\hline
\end{tabular}

up to $22 \%$ of the stellar mass of I Zw 18 could be contributed by older stars.

\subsection{Discussion}

The five-level analysis using the NEBULAR package revealed that the oxygen abundances of the candidate XMPGs were not nearly as low as expected given their anomalously low $[\mathrm{N} \mathrm{II}] / \mathrm{H} \alpha$ ratios. The line ratio methods were for the most part in agreement with the more extensive analysis. The discrepancies can largely be attributed to the application of the index outside the recommended metallicity range.

Still it is very curious that the oxygen abundances are as high as they are in the three best XMPG candidates when the $[\mathrm{N}$ II] $/ \mathrm{H} \alpha$ ratios are so unique. One possible clue is the ratio of nitrogen to oxygen. Dufour, Garnett \& Shields (1988) measure the nitrogen abundance of $\mathrm{I} \mathrm{Zw}$ 18. They find that $12+\log (\mathrm{N} / \mathrm{H})=5.99$ and that the nitrogen to oxygen ratio is $\log (\mathrm{N} / \mathrm{O})=-1.25$. The nitrogen abundances measured for the XMPG candidates are 5.89 and 5.10 for SDSS J0947-0005 and SDSS J1543+0112 respectively. The 
ratio $\mathrm{N} / \mathrm{O}$ is assumed to be the same as $\mathrm{N}^{+} / \mathrm{O}^{+}$(Peimbert \& Costero 1969). The ratio $\log (\mathrm{N} / \mathrm{O})=-1.73$ and -1.84 . The nitrogen abundance of SDSS J0947-0005 is in good agreement with $\mathrm{I} \mathrm{Zw}$ 18. Given the large uncertainty in the [N II] line at $6584 \AA$ in SDSS $\mathrm{J} 1543+0112$ the nitrogen abundance may be off, but it is undoubtedly low and probably similar to that of SDSS J0947-0005 and I Zw 18.

There is a notable difference in $\log (\mathrm{N} / \mathrm{O})$ between $\mathrm{I} \mathrm{Zw} 18$ and the two XMPG candidates found with 2dF. Both SDSS J0947-0005 and SDSS J1543+0112 have N/O ratios which are at least 6 times lower than I Zw 18. Storchi-Bergmann, Calzetti, \& Kinney (1994) find that the relationship between $\log (\mathrm{N} / \mathrm{O})$ and $12+\log (\mathrm{O} / \mathrm{H})$ is linear for galaxies with oxygen abundances large than 8.3. However van Zee, Salzer, \& Haynes (1998) that the relationship has substantial scatter at lower oxygen abundances. They attribute this spread to the physical origin of the nitrogen.

Nitrogen production can be either primary or secondary and is described in VilaCostas \& Edmunds (1993). Nitrogen is generally thought to be a byproduct of the CNO cycle in the cores of hydrogen burning stars. If oxygen and carbon are contained in a star at its formation and a constant mass fraction is processed than the amount of nitrogen will be proportional to the initial abundance of carbon and oxygen. In these stars nitrogen production is referred to as secondary. Carbon and oxygen can also be produced by the star, such as in core helium burning. The carbon and oxygen produced in this manner can then migrate to a hydrogen burning shell resulting in nitrogen production by the CNO cycle. This scenario is called primary nitrogen production. The amount of nitrogen produced in the primary model can be more or less independent of the initial abundances. Secondary 
production is thought to happen in stars of all masses where primary production is thonght to happen in intermediate mass stars (although there is still some debate on this topic).

The signature of secondary nitrogen is that $\log (\mathrm{N} / \mathrm{O})$ continues to decrease linearly as the oxygen abundance decreases. For primary nitrogen the relationship starts as a linear trend at high abundance but flattens out to a constant value at low metallicities. Plotting the values of I Zw 18, SDSS J0947-0005, and SDSS J1543+0112 on the figure in van Zee, Salzer, \& Haynes (1998) reveals that I Zw 18 falls on the primary production track while the other two galaxies fall on the exclusively secondary production track with lower $\log (\mathrm{N} / \mathrm{O})$ values than any of the galaxies and $H$ II regions in the figure. In fact Thuan, Izotov, \& Lipovetsky (1995) have a similar plot for a sample of blue compact galaxies, including I $\mathrm{Zw}$ 18. For their blue compact galaxies there is little scatter and the $\log (\mathrm{N} / \mathrm{O})$ values remain constant with decreasing oxygen abundance. They attribute this to primary production of nitrogen in BCGs. Infall and outflow of gas can also give rise to constant $\log (\mathrm{N} / \mathrm{O})$. However van Zee, Salzer, \& Haynes (1998) argue that this is not likely to be the cause of the constant $\log (\mathrm{N} / \mathrm{O})$ in dwarf galaxies as evidence of primary nitrogen is seen in higher mass spirals with deeper gravitational potential wells where outflows are not as large of an effect.

The fact that nitrogen appears to be secondary in these objects may be further evidence that an older stellar population exists in these objects. However it may also be due to a time delay in the relcase of oxygen in nitrogen. If nitrogen is produced predominantly in intermediate mass stars then there will be a period of low $\mathrm{N} / \mathrm{O}$ ratios after the massive stars release their oxygen and the intermediate mass stars begin releasing nitrogen. It may 
also be that these two galaxies have been caught at this precise moment.

While it was briefly thought that the quasar selection of Chiu (2006) might be a useful selection for XMPGs it clearly is not. First of all only two XMPG candidates were found among the $\sim 24,500$ objects studied. Secondly those candidates in the end turned out to have low metallicities, but not the extremely low metallicities that were initially suspected. However it appears that the two galaxies found in the quasar survey are unique in that they have particularly low nitrogen abundances compared to their oxygen abundances. This suggests that nitrogen is almost exclusively produced as a secondary element. This is somewhat of a mystery as most blue compact dwarfs appear to have a substantial fraction of nitrogen produced as a primary element. In addition these two galaxies suggest that line ratio metallicity approximations which are include nitrogen lines, in particular the $[\mathrm{N}$ II] line at $6584 \AA$ may not be robust for galaxies with low oxygen abundances. This could potentially cause problems for studies of metallicities at high redshifts where oxygen abundances will be on average lower and metallicity measurements will rely more heavily on line ratio measurements due to the difficulty of observing these objects. 


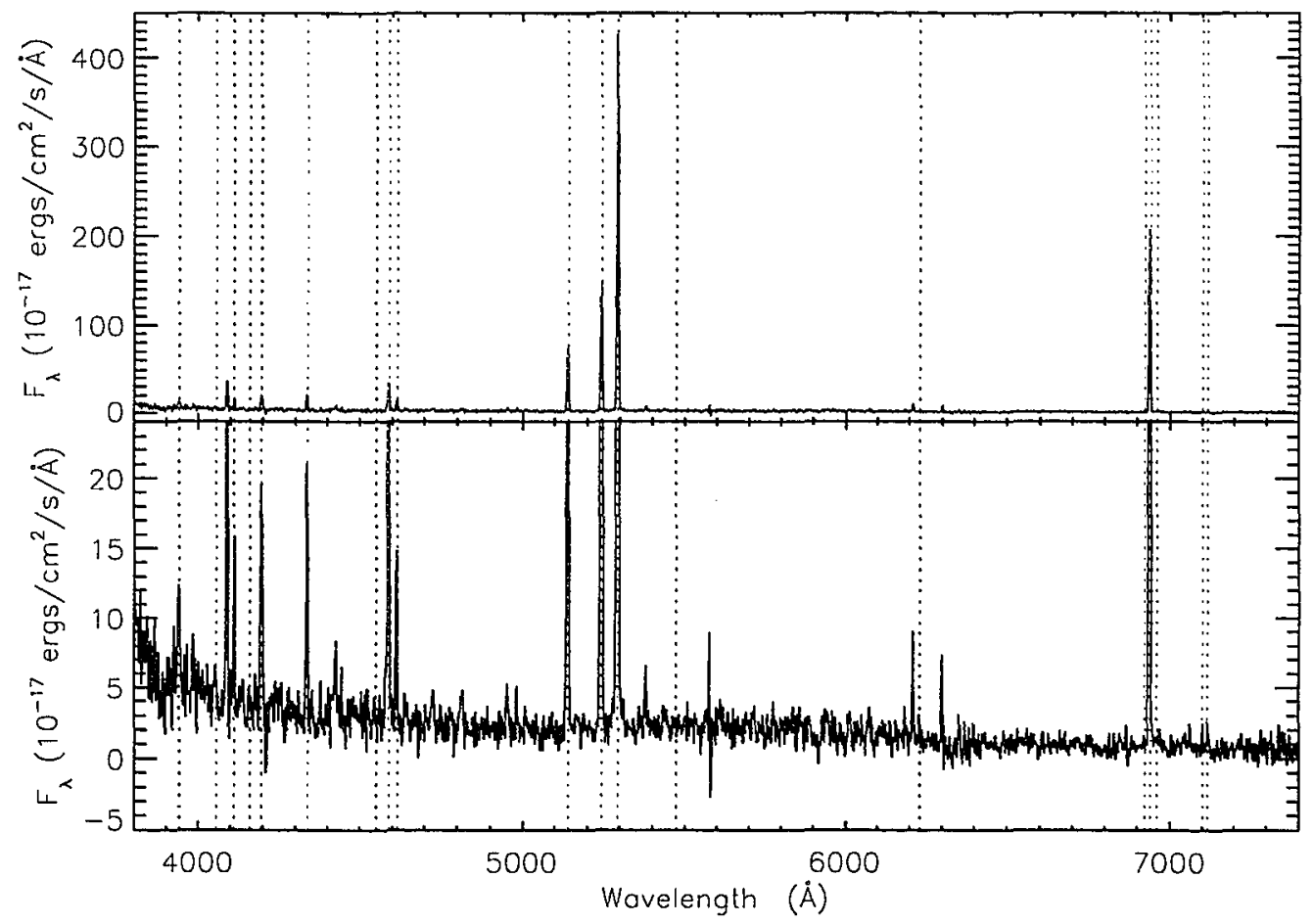

Figure 6.1 Spectrum of the low metallicity galaxy SDSS J094725.23-000509.3. The spectrum was taken with the Double Beam Spectrograph on the 2.3 Advanced Technology Telescope at Siding Spring Observatory outside Coonabarabran, New South Wales, Australia. The spectra are identical in the top and bottom panels. However the stretch in flux differs in order to show the strongest emission lines (top panel) and also weak lines and the continuum (bottom panel). The dotted lines show the position of known emission and absorption lines shifted to $z=0.0568$, the redshift of SDSS J0947-0005. The magenta lines show the positions of Balmer series lines in hydrogen, cyan show the positions of metal emission lines, and green show the positions of stellar absorption features. 


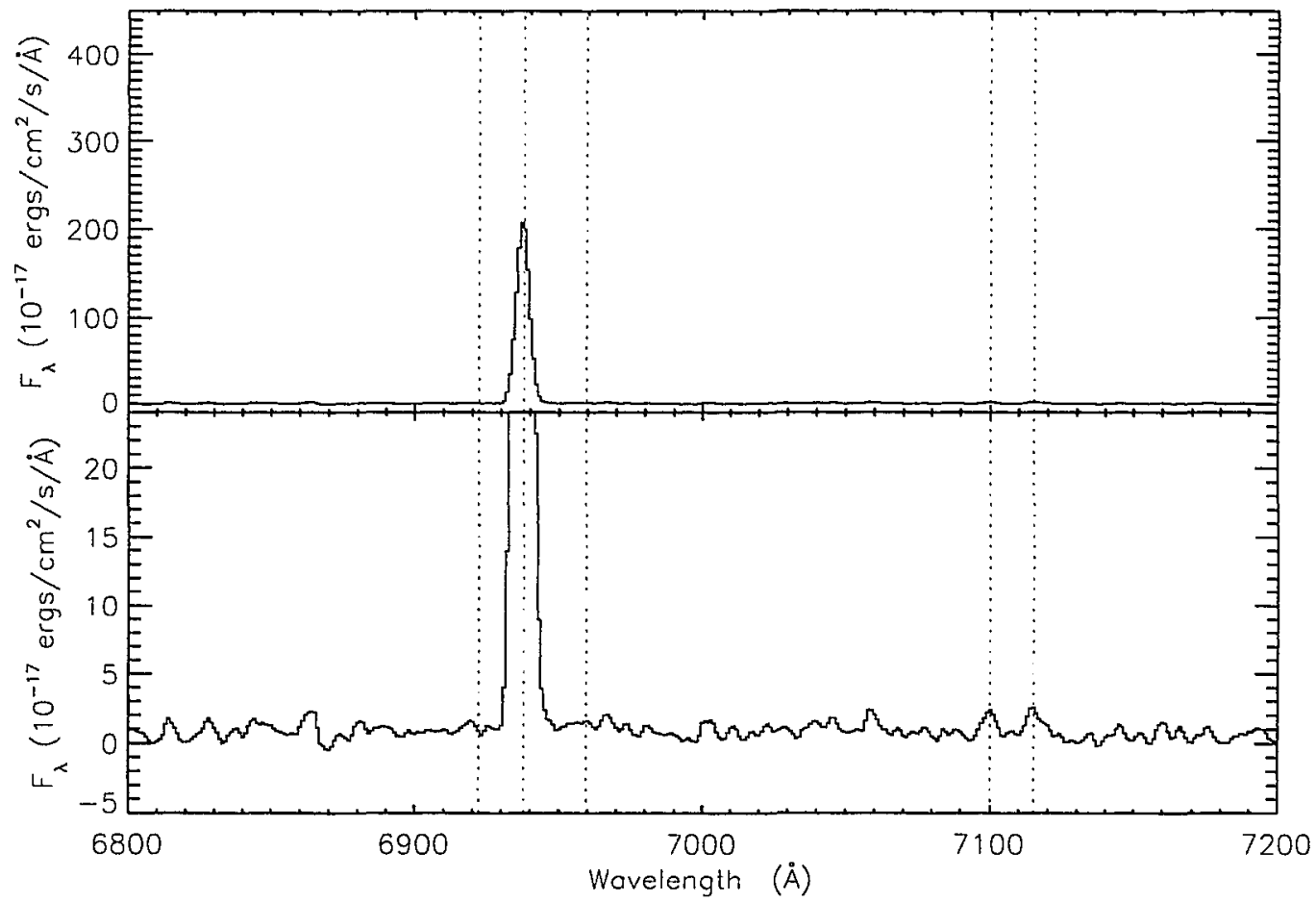

Figure 6.2 Close up of the spectrum of the low metallicity galaxy SDSS J094725.23-000509.3 around the $\mathrm{H} \alpha$ emission line. The description follows that of Figure 6.1 although the wavelength range has been narrowed for a clearer view of the $\mathrm{H} \alpha$ line redshifted to $6940 \AA$, the [N II] lines on both sides of $\mathrm{H} \alpha$, and the [S II] doublet redshifted to $7100 \AA$. 


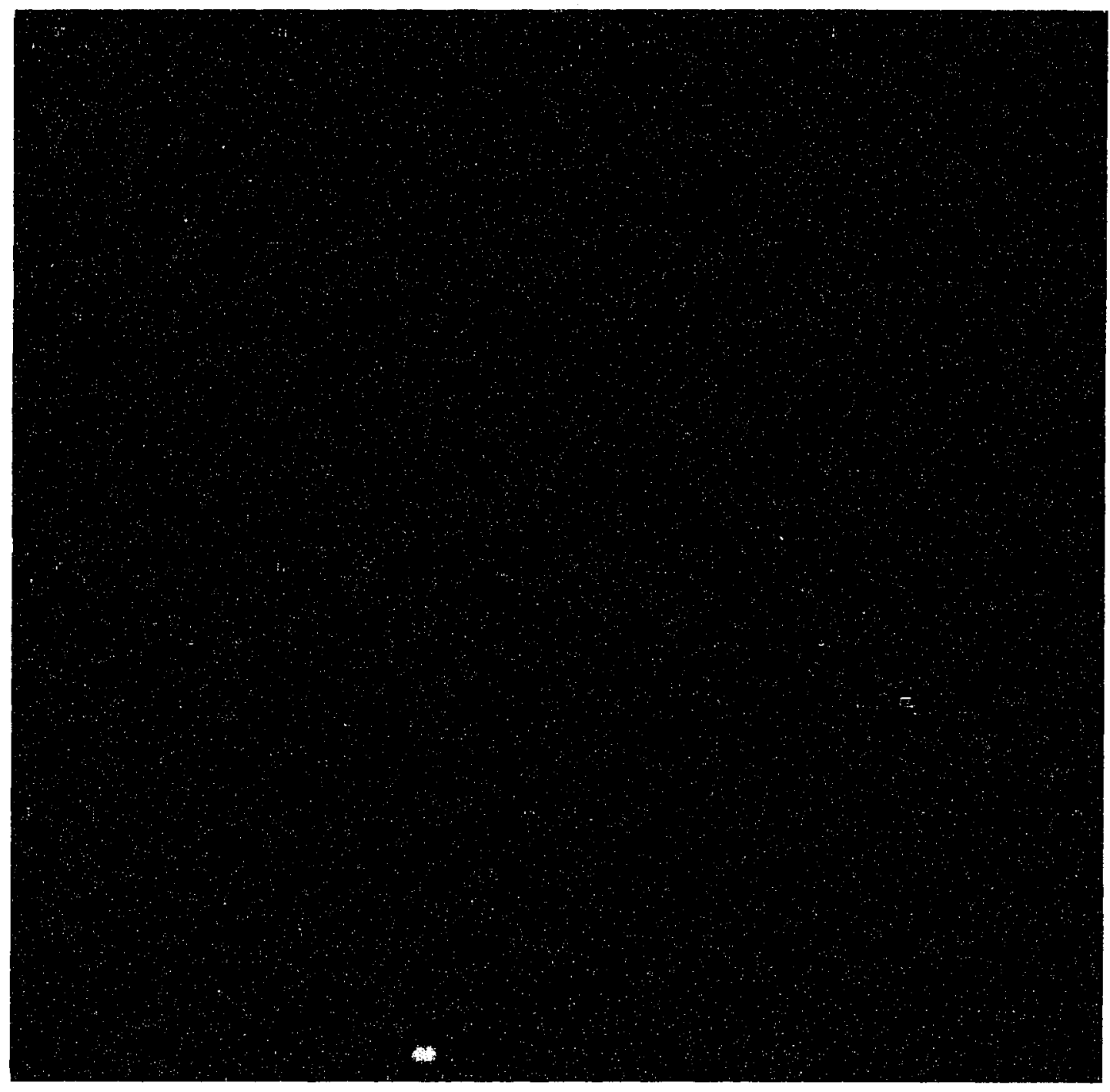

Figure 6.3 SDSS images of XMPG candidates. Upper left: I Zw 18, one of the lowest metallicity galnxies known. The grid is centered on the NW part of I Zw 18 which is the most metal poor. Comparing this image with Figure 1.5 reveals the relative power of SDSS and HST. Upper right: SDSS J0947-0005. Lower left: SDSS J1543+0112. Lower right: SDSS J0239-0031. The horizontal bar in the upper left of each panel is 5" long. 


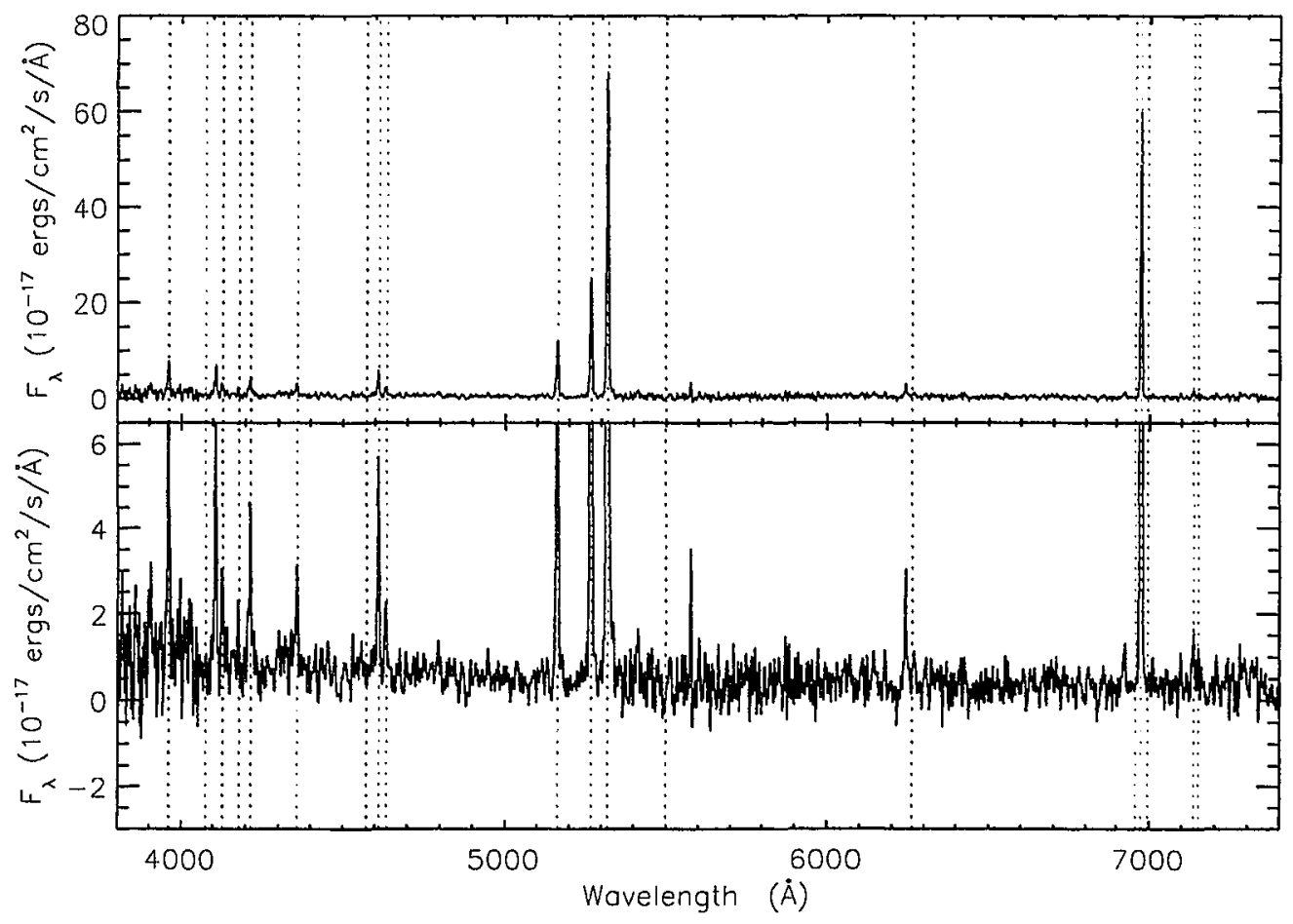

Figure 6.4 Spectrum of the low metallicity galaxy SDSS J154311.30+011235.4. The spectrum was taken with the Double Imaging Spectrograph II instrument on the ARC $3.5 \mathrm{~m}$ telescope at Apache Point Observatory in New Mexico. The description follows that of Figure 6.1. The redshift of SDSS J1543+0112 is $z=0.0621$. 


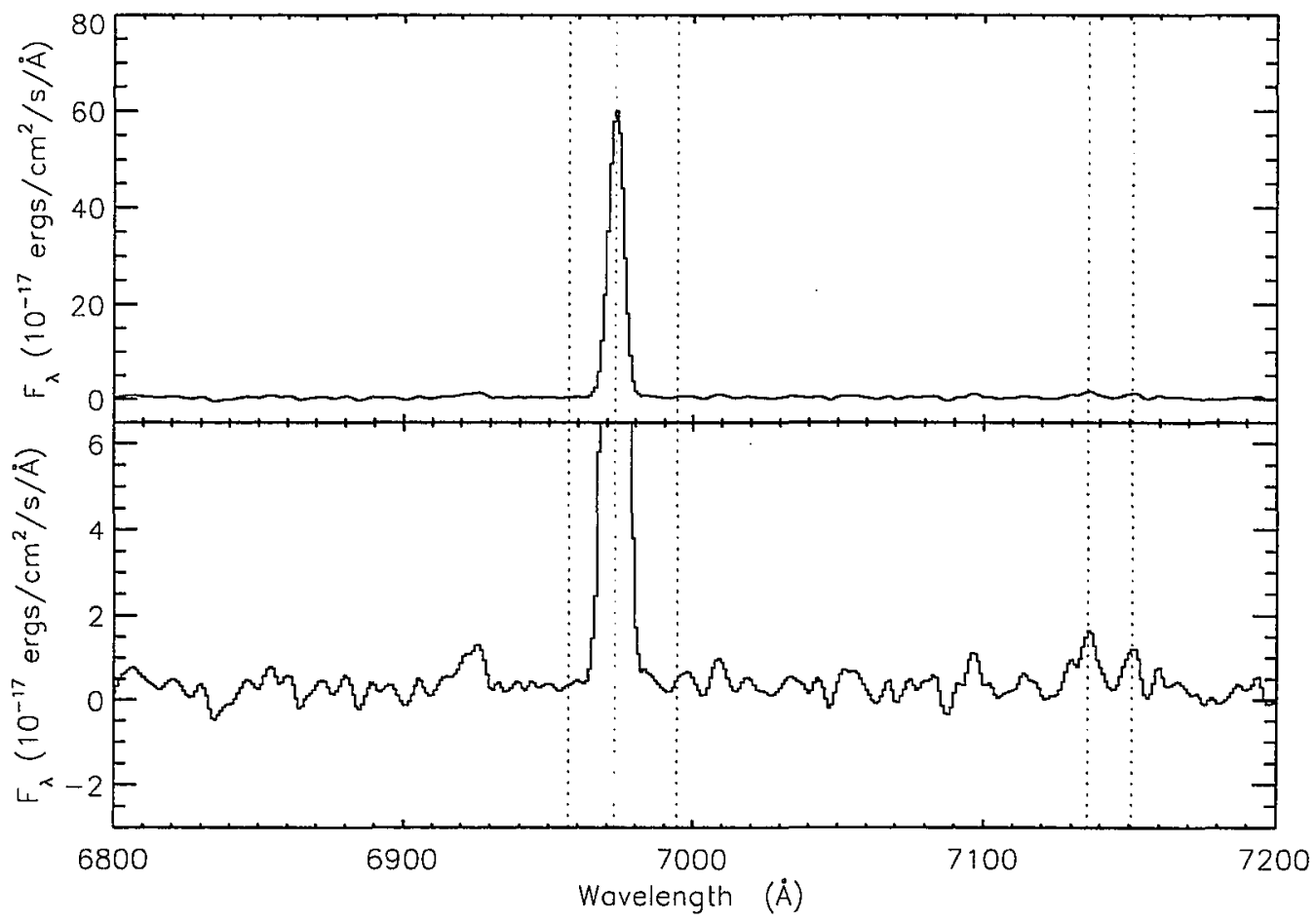

Figure 6.5 Close up of the spectrum of the low metallicity galaxy SDSS J154311.30+011235.4 around the $\mathrm{H} \alpha$ emission line. The description follows that of Figure 6.1 although the wavelength range has been narrowed for a clearer view of the $\mathrm{H} \alpha$ line redshifted to $6970 \AA$, the [N II] lines on both sides of $\mathrm{H} \alpha$, and the [S II] doublet redshifted to $7140 \AA$. 


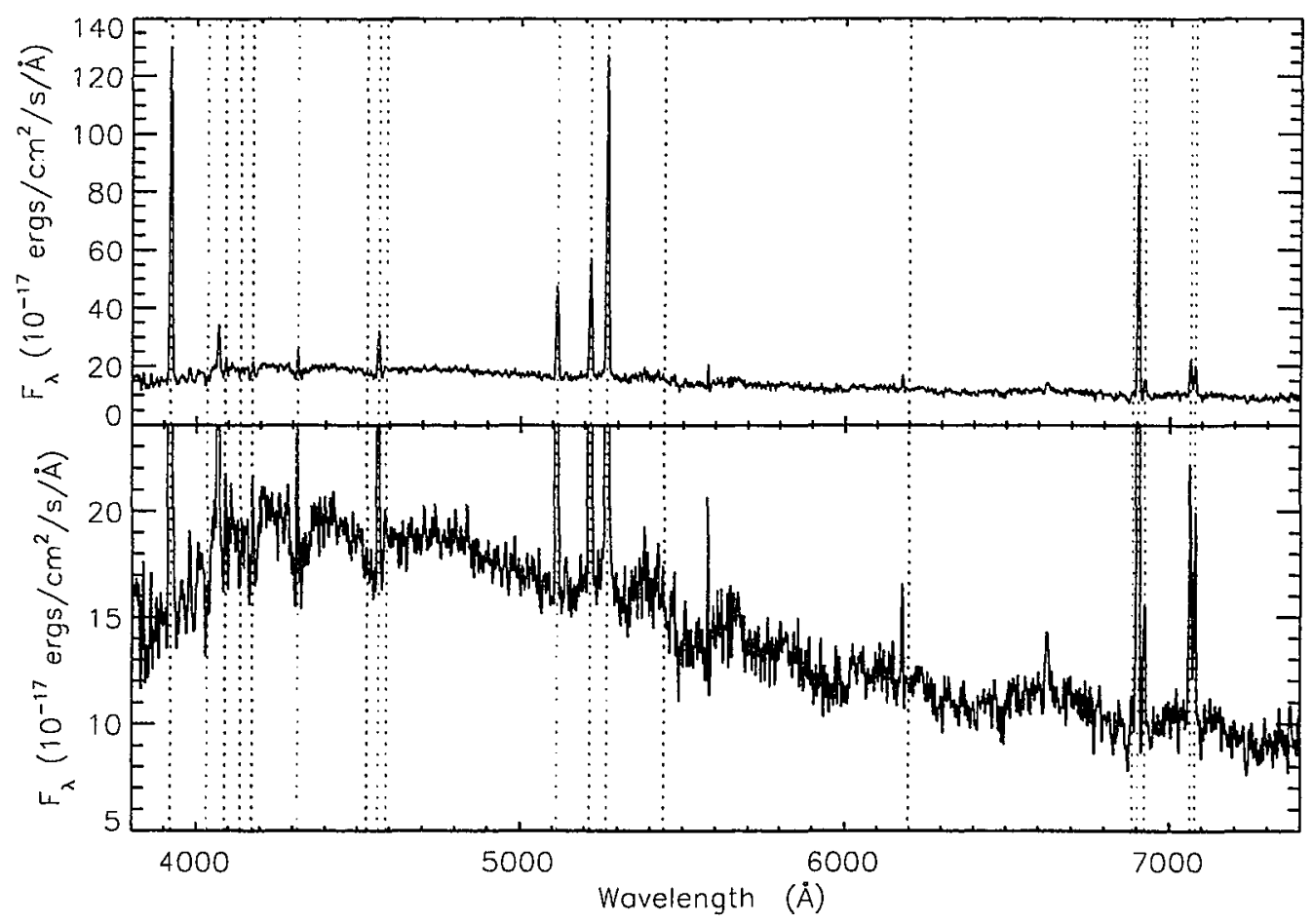

Figure 6.6 Spectrum of the galaxy SDSS J023958.57-003149.1. The spectrum was taken with the Double Imaging Spectrograph II instrument on the ARC 3.5m telescope at Apache Point Observatory in New Mexico. The description follows that of Figure 6.1. The redshift of SDSS J0239-0031 is $z=0.0513$. 


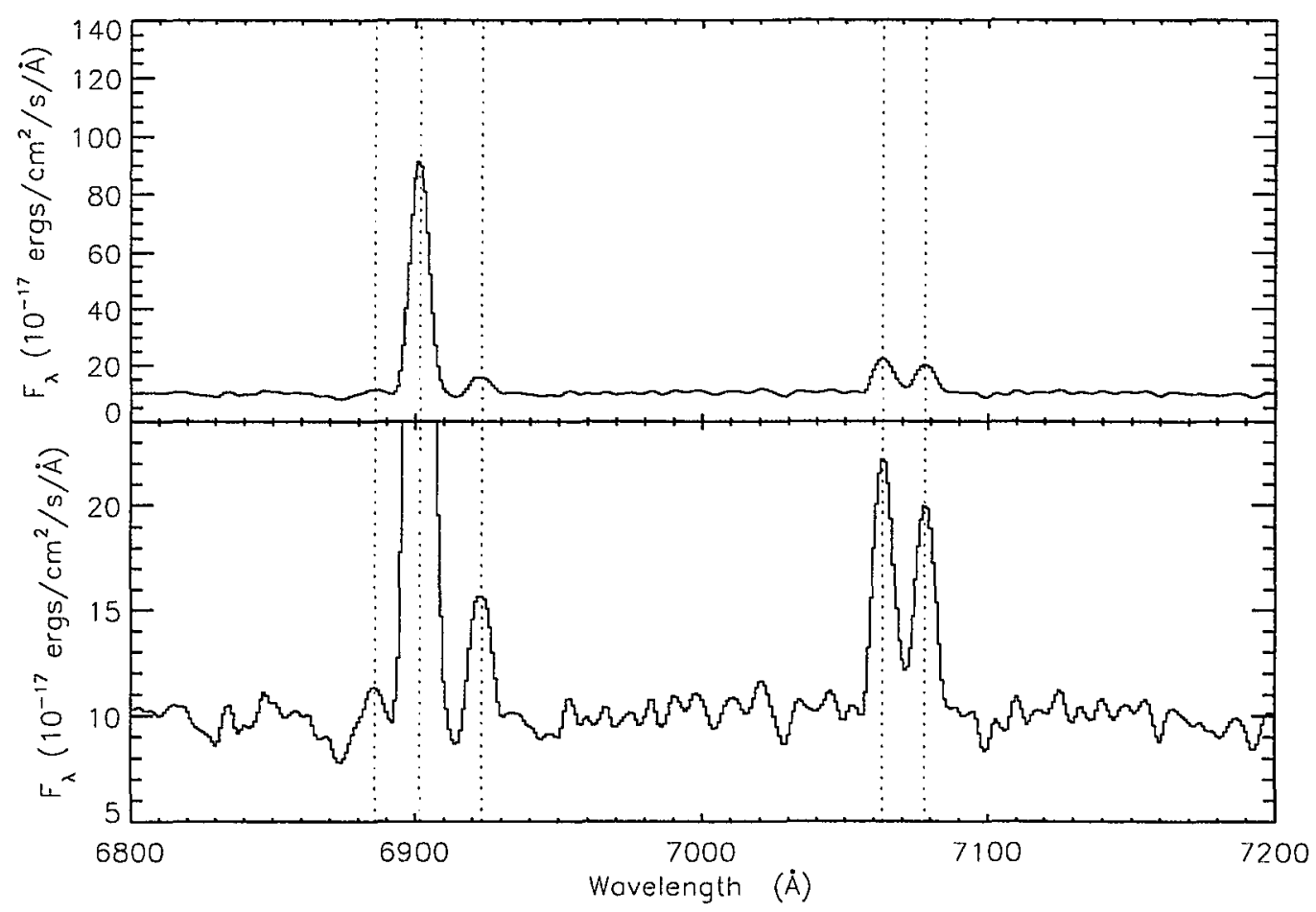

Figure 6.7 Close up of the spectrum of the galaxy SDSS J023958.57-003149.1 around the $\mathrm{H} \alpha$ emission line. The description follows that of Figure 6.1 although the wavelength range has been narrowed for a clearer view of the $\mathrm{H} \alpha$ line redshifted to $6900 \AA$, the [N II] lines on both sides of $\mathrm{H} \alpha$, and the [S II] doublet redshifted to $7070 \AA$. Note that the [N II] lines are far more prominent than in Figures 6.2 and 6.5 which suggest that the metallicity of this galaxy is not as low as those in the other figures. 


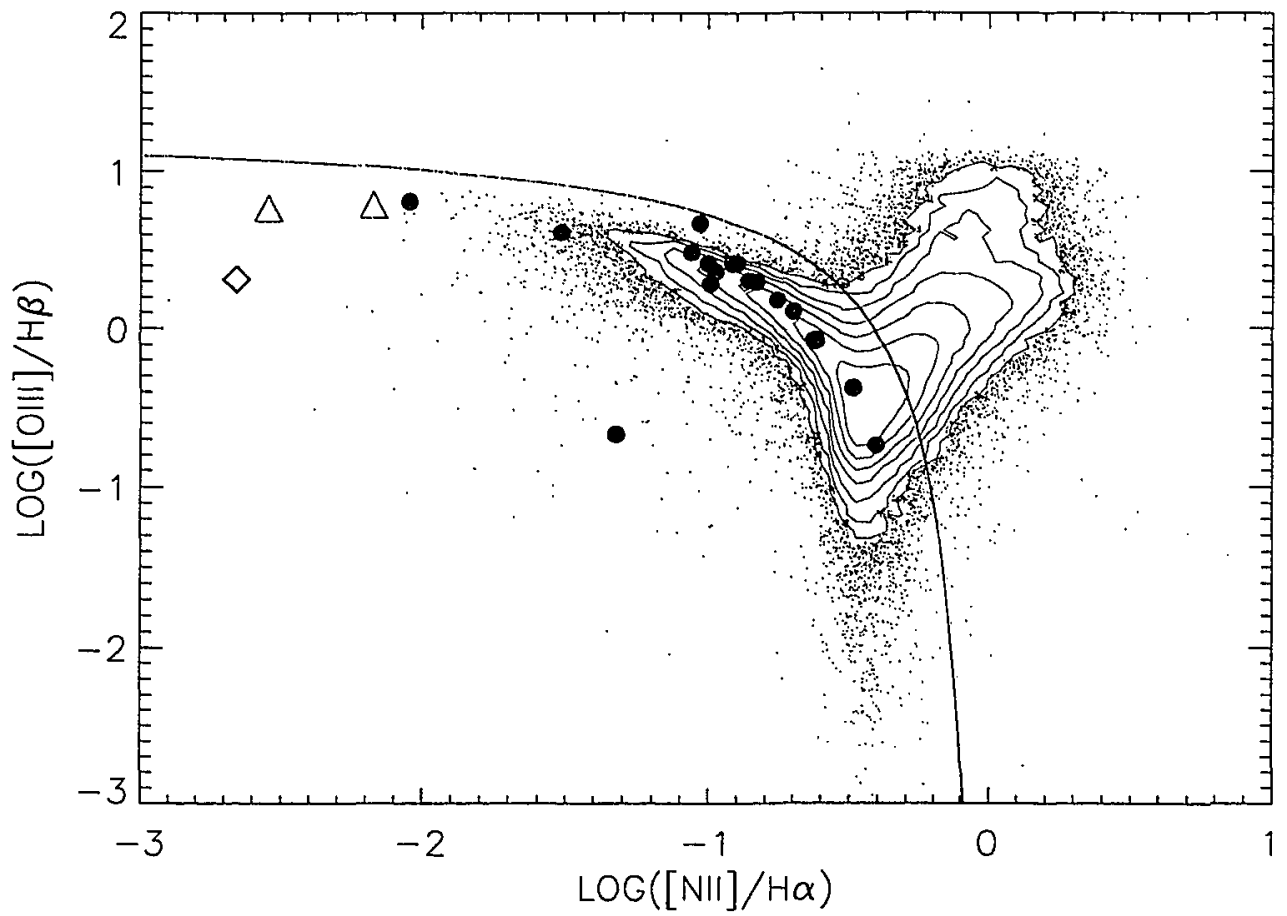

Figure 6.8 BPT diagram of low metallicity galaxy candidates. The BPT diagram uses the logarithms of the $[\mathrm{N} \mathrm{II}] / \mathrm{H} \alpha$ and $[\mathrm{O}$ III $] / \mathrm{H} \beta$ emission lines to test for AGN activity. The contours and small points show the positions in the BPT diagram of 204,494 objects in SDSS DR4 with $\mathrm{S} / \mathrm{N}>5$ in both the $\mathrm{H} \alpha$ and $\mathrm{H} \beta$ emission lines. The solid green circles show the positions of the candidate low metallicity galaxies from the SDSS spectroscopy. The magenta triangles are the XMPG candidtes from the 2dF quasar survey and the blue diamond is the known XMPG I $\mathrm{Zw}$ 18. The red line separates AGN from star forming galaxies (Kauffmann, et al. 2003). The location of the green points below and left of the red lines indicates that the candidate low $\mathrm{Z}$ galaxies are star forming galaxies with minimal AGN activity, if any. 

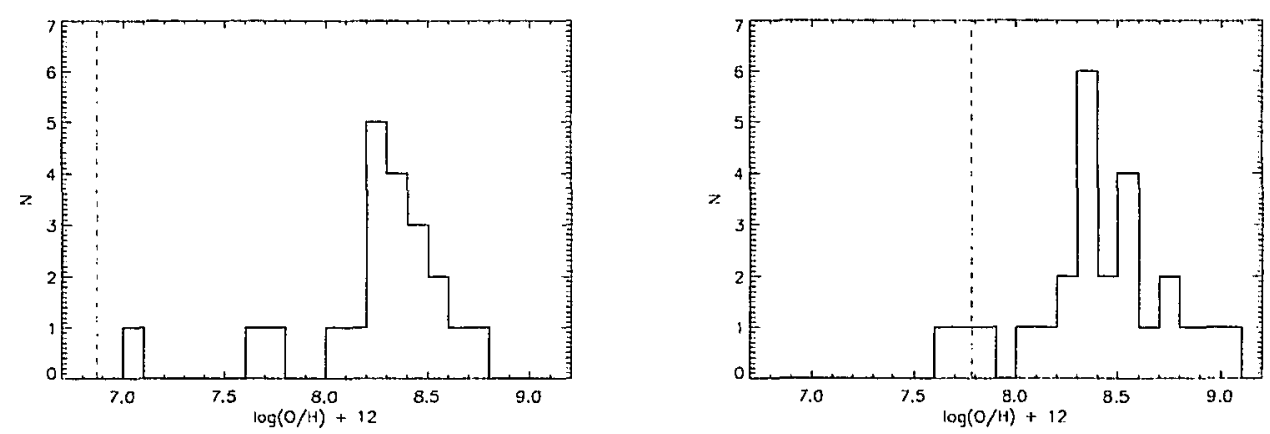

Figure 6.9 Histograms of candidate galaxy metallicities as measured from the $[\mathrm{N} \mathrm{II}] / \mathrm{H} \alpha$ (left panel) and O3N2 (right panel) ratios.

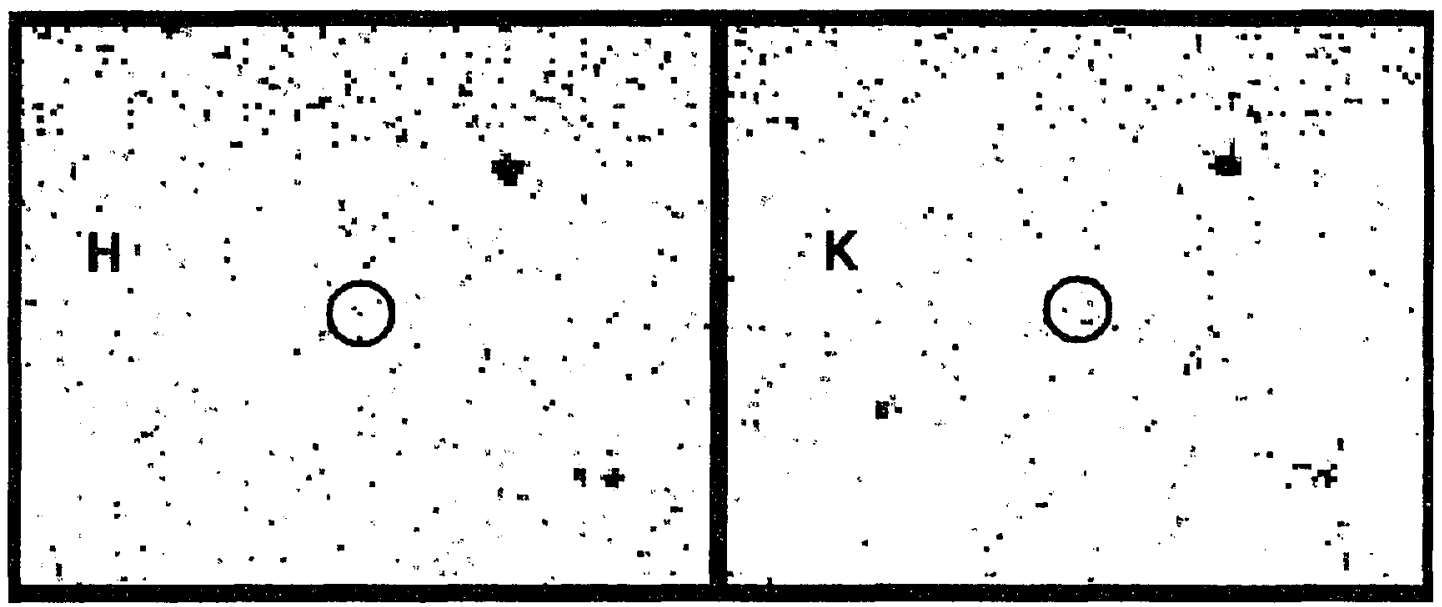

Figure 6.10 $\mathrm{H}$ and $K_{S}$ images of the low metallicity galaxy SDSS J094725.23-000509.3. Imaging was done with the GRIM II camera on the ARC $3.5 \mathrm{~m}$ telescope at Apache Point Observatory in New Mexico. At left is the $H$ band image and at right is the image in the $K_{S}$ band. The exposure time is $60 \mathrm{~s}$ in both bands. The green circle in each panel shows the SDSS J0947-0005 is undetected in $H$ and has $K_{S}=19.68 \pm 0.43$. For reference the object in the lower right of each panel has $H=20.32 \pm 0.28$ and $K_{S}=19.56 \pm 0.38$. For reference the SDSS mosaic image can be seen in the upper right of Figure 6.3. 


\section{Chapter 7}

\section{Conclusions}

While the three projects described in this thesis may seem disparate they all share a common theme. All three harness the power of the unprecedented size of the SDSS in seeking new insights into galaxy formation. This is a relatively new way of doing business in astronomy, made possible by several technological advances. Perhaps most important are advances in computing. Advances in data storage make it possible to store the terabytes of data generated by the SDSS and increases in RAM and CPU speed have made it possible to reduce and analyze large amounts of data in meaningful and cost effective ways on desktop machines. In addition relatively inexpensive supercomputers can be built by joining desktops together and programs can be written to exploit these parallel computing possibilities. CCDs are continually being improved with smaller pixel sizes for better spatial resolution, more uniform response across the chip, larger arrays for more information per pointing, and reductions in dark currents and read noise. Multi-object spectrographs have greatly reduced the amount of time to do spectroscopy. Where at one time objects needed to be observed one at a time using long-slit spectroscopy today instruments like $2 \mathrm{dF}$ and SDSS can simultaneously take spectroscopy of 400 and 640 objects respectively. 
None of this was possible in the age when photographic film was the currency of observations and computers ran on punch cards. In many ways astronomy is beginning a transition much like the one experimental particle physics is several decades into. Gone. are the days of individual labs building their own bubble chambers and measuring events one at a time from painstakingly measuring particle paths from photographs. Today huge collaborations build multi-billion dollar accelerators and banks of computers analyze millions of events per second. Automated programs decide which events eventually receive the attention of individual scientists.

Similarly with the growth of large scale survey projects the study of astronomy is headed the same way. These projects are so large in scale that they take teams of people to get them off the ground. They are expensive to undertake. The SDSS built its own dedicated telescope and specialized software was written for the survey. In addition the amount of data is so immense that no one person could possibly ever look at it all. The SDSS DR6 contains 287 million unique objects. If an astronomer spent just one second looking at each object it would take them 9 years and 2 months without sleeping or 39 years and 10 months of working regular business hours to see the whole survey. Given that the SDSS continues to grow one could never hope to catch up.

For the romantic inside of us it is sad to see the Hollywood vision of the astronomer fall by the wayside. There is something about the image of the bearded, spectacled, professor with corduroy patches on his blazer sitting alone on a lentote nountain pecring through a large refracting telescope in lonely pursuit of gleaning truth from the heavens. However the new age of astronomy provides a wealth of exciting new opportunities. 
One way in which large surveys provide new insights is in the discovery of increasingly rare objects. With over 1.2 million spectra observed in SDSS DR6 objects which are truly one in a million can be found. Unlike other branches of physics where laboratory experiments can be built to design and carefully controlled astrophysicists must rely on observing what already exists. It is hopelessly impossible to create in the lab the high temperatures, density extremes, magnetic fields, or gravitational forces which can exist in astrophysical contexts let alone build your own star or galaxy to order. Oddball objects can expand the known parameter space of a class of objects or provide the missing link in the evolution from one class to another.

Within this thesis two blue compact dwarf galaxies were found with anomalously low nitrogen contents given their oxygen abundances. This could potentially provide clues into their stellar populations or dynamics which set them apart from the general population and ultimately shed new light on the chemical evolution of galaxies. A quasar, originally believed to be a lensed LBG, with only Ly $\alpha$ emission was also uncovered in the SDSS. This was thought not to be a particularly rare object but rather a chance precise geometrical alignment which affords a view of a normal object from a angle not seen before.

This brings up a problem faced every fraction of a second by the high energy physicists. How does one throw away all of the pieces of hay while being certain no needles are lost? Conversely how do you keep all the needles without keeping so much hay that finding the necdles is still prohibitively difficult? In high encrgy woll studicd particle production events need to be filtered out without accidently throwing out new decay routes which are interesting. Chapter 4 deals with this problem from an astronomical perspective. 
Normal galaxies need to be filtered out to leave only those which are truly rare objects. The additional concern of Chapter 4 deals with pushing the limits of a survey as far as possible to probe objects with poor $\mathrm{S} / \mathrm{N}$. In addition statistics can work against you. A catalog of 287 million objects is expected to have over $750,0003 \sigma$ events and $2005 \sigma$ events just from statistical fluctuations. However with such huge data sets exciting new objects can be appear even when they are not being sought. The objects with low nitrogen to oxygen ratios were found serendipitously as part of study of high redshift quasars.

The second way in which large surveys can deepen understanding is by providing statistically robust samples from which the mean properties of a group of objects can be measured. With increasing large surveys more narrow sub-samples can be defined yet still have the numbers necessary for a detailed analysis. In Chapter 3 the IMF was measured in very narrow luminosity bins. This was made possible by the sample size of 130,602 galaxies. Furthermore at a given luminosity galaxies could be binned by extinction, redshift, or aperture fraction to search for potential biases. While measuring the SFHs of individual unresolved galaxies is next to impossible to do with certainty the large sample size made it possible to show that the necessary distribution of SFHs to match the observations is improbable - a view of the forest not possible from looking at individual trees.

From a technical standpoint this thesis focussed on the implementation of existing techniques for working with large data sets and, where necessary, developing new ones. The science goals focussed on gaining new insight into galaxy formation and cvolution.

One of the avenues pursued in this goal was to try to exploit opportunities to see into the pasts of galaxies. In the case of the search of LBGs the idea was to look for star 
forming galaxies at such large distances that the light arriving from them here today left them while they were still in their youths. Generally these objects are too faint, given the large distances involved, to be observed in depth. However chance geometrical alignments with massive intervening galaxy clusters can magnify them via gravitational lensing allowing for high $\mathrm{S} / \mathrm{N}$ spectroscopy on the world's largest telescopes. If strongly lensed LBGs could be found in a systematic way then a wealth of information regarding the stellar populations, chemical emrichment, and dynamics of the progenitors of today's galaxies could have been found. As it is no strongly lensed LBGs were found so the principle science goals could not be met. However a great deal was learned from looking for them.

Another way to look into the pasts of galaxies is to try to find objects which are frozen in time-- relics of a past age. These objects for some reason did not age like other galaxies. These galaxies, should they exist, would be marked by extremely low metallicities due to the fact that the onset of star formation was mysteriously delayed until the present day. Finding such objects could provide insights into the first stars which formed in metal free environments. Much is theorized but little is known about these stars which played a significant role in reionizing the Universe and are thus of great importance to the understanding of cosmology. These present day primordial galaxies could provide clues into galaxy formation as well if the conditions that caused the onset of star formation could be understood, as well as the conditions that retarded star formation until now.

Two blue compact dwarf galaxies which were initially thought to fit this description were discovered serendipitously as part of a quasar survey. Abundance analysis revealed that these two galaxies have metallicities which are low, but not remarkably so. In addition 
an old stellar population was detected in near infrared imaging. However it was found that the nitrogen to oxygen ratio was significantly lower than is the norm for blue compact dwarf galaxies. This is likely due to a difference in the production mechanism of nitrogen and suggests that the underlying stellar population and history of the two galaxies differs from the norm in such objects. Furthermore it could provide more general insights into the production of nitrogen and the enrichment of galaxies.

From a more practical standpoint the existence of low metallicity galaxies with low nitrogen to oxygen ratios could plague the study of the chemical evolution in the high redshift universe. Galaxies in the early universe, due to the large distances involved, are faint and difficult to observe. The menagerie of weak emission lines used for a full abundance analysis cannot practically be measured with good $\mathrm{S} / \mathrm{N}$ if at all. The common way to get around this is to use line ratios of well detected emission lines as proxies for metallicity. These relationships between these line ratios and metallicity are calibrated against nearby galaxies and $\mathrm{H}$ II regions which are well studied. Given that the nitrogen to oxygen ration may not follow as simple of a relationship at low oxygen abundances as it does at more standard abundances line ratios which include nitrogen lines may be systematically incorrect for low metallicity galaxies in the high redshift universe. This could potentially cause problems for studies of chemical enrichment in galaxy evolution.

While the results in this thesis pose more questions than answers regarding the production mechanisms for nitrogen they porhaps act as a cautionary tale to those using line ratio metallicity indicators on low metallicity galaxies.

From both a technical and scientific perspective the crowning achievement of this 
thesis is the results of the IMF study. Systematic variation of the IMF with luminosity was observed which cuts against the conventional wisdom that the IMF is universal regardless of environmental conditions. In the high luminosity galaxies a Salpeter-like IMF slope was recovered and these galaxies were well represented by a single, universal IMF. However it was found that in low luminosity galaxies the IMF has fewer massive stars and that a single IMF is not a good fit to the population. There is some controversy whether these results are caused by variations in the stellar IMF. It may also be possible that the stellar IMF is universal, but the way in which it is sampled by molecular clouds, which may have their own power law distribution, causes the IMF integrated over the whole galaxy appear to vary. It is far from clear at this point which interpretation is correct. Regardless the results here indicate that when modeling low luminosity galaxies a Salpeter IMF may not be appropriate. This may also affect models of the early chemical enrichment of today's galaxies as they assembled from smaller dwarf galaxies.

However these results are tempered by the potential systematic effects introduced by uncertainties in the spectral synthesis models. These uncertainties are primarily due to the poorly understood evolution of the most massive stars and those on the AGB and horizontal branch. The level of these uncertainties is not yet well known. A good, quantitative estimate of this uncertainty is one of the main issues which needs to be resolved before a more definitive claim of IMF variations can be made.

From a technical stanclpoint the use of large sample sizes to address the star formation history of galaxies is a significant accomplishment. The star formation history of a galaxy is notoriously difficult to measure, especially if the stellar population is unresolved. 
However by analyzing the distribution of SFHs needed to account for the observations it: was shown that an unreasonable coordination of star burst times is required. While the problem of measuring individual star formation histories remains in certain circumstances it can be solved for populations of objects.

\subsection{Future Work}

None of the problems addressed in this thesis have been fully resolved. In fact, many new questions arose from these projects. In this section the potential future direction of these projects is discussed.

The targeted search for strongly lensed LBGs came up empty handed. Given the amount of effort put into the search here as well as by many others it is clear that finding these objects in the SDSS is difficult. Looking back the feeling is that the SDSS may be about one magnitude to shallow for a targeted photometric search to be successful. In addition it appears that Allam, et al. (2007) may be having success pulling additional lensed LBGs out of the SDSS using their catalog of candidate interacting galaxies form the imaging survey. This is somewhat contrary to the original design of this project, which was in part an attempt to find objects lensed by galaxy clusters at distances large enough to be previously undetected. Continuing in this direction would not conflict with the Allam, et al. (2007) effort.

Given the lack of success and the work involved it does not appear that this project should continue. However many lessons were learned along the way about rejecting contaminants and pushing the limits of a survey. These could be applied to new surveys as 
they come down the road to search for lensed LBGs or any other type of rare object.

As for the galaxies with low nitrogen to oxygen ratios the first step in the followup would be to obtain higher $\mathrm{S} / \mathrm{N}$ spectra, and perhaps higher resolution to split some of the line doublets, of the two galaxies in order to perform a more complete and precise abundance analysis. A second step would be to conduct a large systematic survey of blue compact dwarfs from the SDSS to determine what fraction of these galaxies have low nitrogen to oxygen ratios and what the full distribution of these ratios is. Given that there are already similar surveys that did not find galaxies like the two described in this thesis the survey would likely need to have a couple hundred galaxies to measure this accurately. This would provide some insight into how perilous it is to use line ratio indicators at low metallicities. It may also be worth taking high resolution images with the Hubble Space Telescope to determine if the environments around these two galaxies harbor any insights into their odd behavior. Both objects appear very isolated in the SDSS imaging. If this project was undertaken it would required considerable modeling of nitrogen enrichment models as well.

Bold claims require extraordinary evidence. The IMF variations described here certainly qualify as a bold claim. As such it is important to continue this investigation to find additional evidence to support or refute these results. One major issue is to quantify the level of uncertainty introduced by the models due to an incomplete understanding of the evolution of the most massive stars and AGB and horizontal branch stars. Future work on the IMF can proced in a couple of now directions. The first is to continuc to scan the parameter space for systematic IMF variations using the current method. Using the line ratio methods described in this thesis gas phase metallicities can be measured and the IMF 
variation due to metallicity can be investigated. Although part of this thesis reveals some concerns about these line ratio metallicity indicators at low metallicities at the metallicities common to the bulk of the SDSS galaxies the use of these line ratios is far less dubious. Using SDSS galaxy cluster catalogs IMF variations can be investigated as a function of galaxy environment.

It would also be very useful to measure the IMF in SDSS star forming galaxies in a fully independent manner using different IMF indicators. One potential way to do this is to use ultraviolet spectroscopy from the GALEX mission to measure the shape and strength of stellar absorption. According to the spectral synthesis models of Leitherer, Robert, \& Heckman (1995) the C IV $\lambda 1550$, Si IV $\lambda 1400$ and N IV $\lambda 1720$ stellar absorption line strengths and profiles are sensitive to the IMF slope and upper mass cutoff. As this method is sensitive only to the high mass stars it is much less affected by the SFH, a large problem for the optical technique used here. One negative is that these lines may be contaminated by the interstellar medium and would need to be disentangled (Shapley et al. 2003).

Lastly the method used here can be expanded to include more observational parameters to more strongly constrain the models. These parameters can be from the optical but it has recently become possible to access data from large surveys from a number of wavelength regimes. An ultraviolet color index can be used as a star formation rate indicator due to the fact that this is a direct measure of the photospheres of massive stars in the galaxics. Unfortunately the dust correction becomes more uncertain in the ultraviolet which can make it difficult to use. In the infrared SWIRE, a Spitzer Legacy Project, has taken MIPS and IRAC data in three nine square degree fields which overlap with the SDSS 
coverage. These wavebands can provide a check on the number of massive stars determined from the H $\alpha$ flux. One concern is that dust may obscure star formation such that it is not detected in the visible and ultraviolet. If the most massive stars spend their entire lifetimes imbedded in nebular regions my current method could lead to the spurious conclusion that the IMF is truncated at some upper mass. MIPS data can constrain dust and the star formation rate and IRAC can constrain stellar masses. In order to analyze all this data at once more advanced statistical techniques will be needed in order to keep computational costs down. Monte Carlo Markov Chain analysis is a promising method for this purpose. 
Appendix A

Spectroscopic Observations of LBG

Candidates 
Table A.1. Spectroscopically Identified LBG Candidates

\begin{tabular}{|c|c|c|c|c|c|c|c|}
\hline Date & Instrument & R.A & $\operatorname{dec}$ & $r$ & z & Type & Comment \\
\hline $7-19-2001$ & $2 \mathrm{dF}$ & 001712.04 & -103322.1 & & & & \\
\hline $7-19-2001$ & $2 \mathrm{dF}$ & 001731.62 & -095131.1 & & & & \\
\hline $7-19-2001$ & $2 \mathrm{dF}$ & 001735.82 & $\begin{array}{llll}-09 & 43 & 34.7\end{array}$ & & & & \\
\hline $7-19-2001$ & 2dF & 001748.04 & -100752.6 & & & & \\
\hline $7-19-2001$ & $2 \mathrm{dF}$ & 001853.18 & -094351.4 & & & & \\
\hline $7-19-2001$ & $2 \mathrm{dF}$ & 001858.44 & $-0905 \quad 29.4$ & & & & \\
\hline $7-19-2001$ & $2 \mathrm{dF}$ & 001947.33 & $-0902 \quad 33.1$ & & & & \\
\hline $7-19-2001$ & $2 \mathrm{dF}$ & 002135.42 & -090730.8 & & & & \\
\hline $7-19-2001$ & $2 \mathrm{dF}$ & 002152.41 & -102931.0 & & & & \\
\hline $7-19-2001$ & $2 \mathrm{dF}$ & 002156.26 & $-1004 \quad 44.8$ & & & & \\
\hline $7-19-2001$ & $2 \mathrm{dF}$ & 002157.66 & -104041.9 & & & & \\
\hline $7-19-2001$ & $2 \mathrm{dF}$ & $0022 \quad 12.13$ & $\begin{array}{lll}-10 & 12 & 07.8\end{array}$ & & & & \\
\hline $7-19-2001$ & $2 \mathrm{dF}$ & 015636.96 & +002051.2 & & & & \\
\hline $7-19-2001$ & $2 \mathrm{dF}$ & 015706.20 & -000648.7 & & & & \\
\hline $7-19-2001$ & $2 \mathrm{dF}$ & 015706.34 & -000400.2 & & & & \\
\hline $7-19-2001$ & $2 \mathrm{dF}$ & 015925.78 & -004806.5 & & & & \\
\hline $7-19-2001$ & $2 \mathrm{dF}$ & 015954.57 & +001533.8 & & & & \\
\hline $7-19-2001$ & $2 \mathrm{dF}$ & 015955.64 & -003533.7 & & & & \\
\hline $7-19-2001$ & $2 \mathrm{dF}$ & 020004.22 & -004042.1 & & & & \\
\hline $7-19-2001$ & $2 \mathrm{dE}$ & 020054.20 & +005621.1 & & & & \\
\hline $7-19-2001$ & $2 \mathrm{dF}$ & 020054.97 & $-0001 \quad 11.5$ & & & & \\
\hline $7-19-2001$ & $2 \mathrm{dF}$ & 020225.44 & -002520.1 & & & & \\
\hline $7-19-2001$ & $2 \mathrm{dF}$ & 144838.41 & +021231.4 & & & & \\
\hline $7-19-2001$ & $2 \mathrm{dF}$ & 144913.63 & +021901.8 & & & & \\
\hline $7-19-2001$ & $2 \mathrm{dF}$ & 144924.55 & +015435.1 & & & & \\
\hline $7-19-2001$ & $2 \mathrm{dF}$ & 144944.70 & +012103.3 & & & & \\
\hline $7-19-2001$ & $2 \mathrm{dF}$ & 145054.00 & +014446.2 & & & & \\
\hline $7-19-2001$ & $2 \mathrm{dF}$ & 145125.64 & +022152.3 & & & & \\
\hline $7-19-2001$ & $2 \mathrm{dF}$ & 145144.98 & +015558.7 & & & & \\
\hline $7-19-2001$ & $2 \mathrm{dF}$ & 145145.10 & +010729.0 & & & & \\
\hline $7-19-2001$ & $2 \mathrm{dF}$ & 145147.15 & +012839.3 & & & & \\
\hline $7-19-2001$ & $2 \mathrm{dF}$ & 145151.06 & +011616.8 & & & & \\
\hline $7-19-2001$ & $2 \mathrm{dF}$ & 145154.76 & +024714.3 & & & & \\
\hline $7-19-2001$ & $2 \mathrm{dF}$ & 145203.05 & +012533.4 & & & & \\
\hline $7-19-2001$ & $2 \mathrm{dF}$ & 145246.27 & +025542.5 & & & & \\
\hline $7-19-2001$ & $2 \mathrm{dF}$ & 145246.81 & +010137.1 & & & & \\
\hline $7-19-2001$ & $2 \mathrm{dF}$ & 145259.36 & +012335.8 & & & & \\
\hline $7-19-2001$ & $2 \mathrm{dF}$ & 145401.05 & +024542.7 & & & & \\
\hline
\end{tabular}


Table A.1 (cont'd)

\begin{tabular}{|c|c|c|c|c|c|c|c|}
\hline Date & Instrument & RA & dec & $r$ & $z$ & Type & Comment \\
\hline $7-19-2001$ & $2 \mathrm{dF}$ & 214921.15 & -070447.0 & & & & \\
\hline $7-19-2001$ & $2 \mathrm{dF}$ & 214944.64 & $-08 \quad 12 \quad 18.4$ & & & & \\
\hline $7-19-2001$ & $2 \mathrm{dF}$ & 214946.14 & -075348.1 & & & & \\
\hline $7-19-2001$ & $2 \mathrm{dF}$ & 215008.09 & $-0742 \quad 17.6$ & & & & \\
\hline $7-19-2001$ & $2 \mathrm{dF}$ & 215105.38 & -065216.8 & & & & \\
\hline $7-19-2001$ & $2 \mathrm{dF}$ & $2151 \quad 13.98$ & $-0706 \quad 11.5$ & & & & \\
\hline $7-19-2001$ & $2 \mathrm{dF}$ & 215128.58 & $\begin{array}{lll}-07 & 40 & 10.8\end{array}$ & & & & \\
\hline $7-19-2001$ & $2 \mathrm{dF}$ & 215147.27 & -081457.0 & & & & \\
\hline $7-19-2001$ & $2 \mathrm{dF}$ & 215321.69 & -065508.9 & & & & \\
\hline $7-19-2001$ & $2 \mathrm{dF}$ & 145326.45 & +015141.0 & & & & \\
\hline $7-19-2001$ & $2 \mathrm{dF}$ & 145121.82 & +010811.8 & & 3.35 & Quasar & \\
\hline $7-19-2001$ & $2 \mathrm{dF}$ & 001714.67 & -100055.5 & & 5.03 & Quasar & \\
\hline $7-21-2001$ & $2 \mathrm{dF}$ & $01 \quad 1607.74$ & -100824.7 & & & & \\
\hline $7-21-2001$ & $2 \mathrm{dF}$ & 011702.37 & -094259.6 & & & & \\
\hline $7-21-2001$ & $2 \mathrm{dF}$ & 011703.96 & -103551.8 & & & & \\
\hline $7-21-2001$ & $2 \mathrm{dF}$ & $01 \quad 1705.78$ & -095904.0 & & & & \\
\hline $7-21-2001$ & $2 \mathrm{dF}$ & $01 \quad 1730.59$ & $-1012 \quad 10.3$ & & & & \\
\hline $7-21-2001$ & $2 \mathrm{dF}$ & 011800.00 & -094513.6 & & & & \\
\hline $7-21-2001$ & $2 \mathrm{dF}$ & 011802.16 & -095209.5 & & & & \\
\hline $7-21-2001$ & $2 \mathrm{dF}$ & 011900.01 & .092211 .2 & & & & \\
\hline $7-21-2001$ & $2 \mathrm{dF}$ & 012010.32 & -091931.6 & & & & \\
\hline $7-21-2001$ & $2 \mathrm{dF}$ & 012014.76 & -101120.8 & & & & \\
\hline $7-21-2001$ & $2 \mathrm{dF}$ & 012052.12 & -093848.3 & & & & \\
\hline $7-21-2001$ & $2 \mathrm{dF}$ & 012054.36 & -094433.2 & & & & \\
\hline $7-21-2001$ & $2 \mathrm{dF}$ & 012057.81 & -091955.3 & & & & \\
\hline $7-21-2001$ & $2 \mathrm{dF}$ & 012059.93 & -101011.7 & & & & \\
\hline $7-21-2001$ & $2 \mathrm{dF}$ & 012113.95 & -103053.2 & & & & \\
\hline $7-21-2001$ & $2 \mathrm{dF}$ & $0121 \quad 18.86$ & -104106.4 & & & & \\
\hline $7-21-2001$ & $2 \mathrm{dF}$ & 012128.82 & -091449.9 & & & & \\
\hline $7-21-2001$ & $2 \mathrm{dF}$ & 012131.96 & -094539.0 & & & & \\
\hline $7-21-2001$ & $2 \mathrm{dF}$ & 012141.78 & -100927.2 & & & & \\
\hline $7-21-2001$ & $2 \mathrm{dF}$ & 012145.33 & -092311.4 & & & & \\
\hline $7-21-2001$ & $2 \mathrm{dF}$ & 0121.54 .76 & -102740.6 & & & & \\
\hline $7-21-2001$ & $2 \mathrm{dF}$ & 012155.50 & -092257.8 & & & & \\
\hline $7-21-2001$ & $2 \mathrm{dF}$ & 012203.60 & -092449.8 & & & & \\
\hline $7-21-2001$ & $2 \mathrm{dF}$ & 012204.02 & -101737.4 & & & & \\
\hline $7-21-2001$ & $2 \mathrm{dF}$ & 012222.65 & -101444.0 & & & & \\
\hline $7-21-2001$ & $2 \mathrm{dF}$ & 012228.90 & -102912.8 & & & & \\
\hline
\end{tabular}


Table A.1 (cont''d)

\begin{tabular}{|c|c|c|c|c|c|c|c|}
\hline Date & Instrument & $\mathrm{RA}$ & dec & $r$ & $\mathrm{z}$ & Type & Comment \\
\hline $7-21-2001$ & $2 \mathrm{dF}$ & 012310.01 & -102225.1 & & & & \\
\hline $7-21-2001$ & $2 \mathrm{dF}$ & 021659.76 & -003252.8 & & & & \\
\hline $7-21-2001$ & $2 \mathrm{dF}$ & 021701.43 & -003440.6 & & & & \\
\hline $7-21-2001$ & $2 \mathrm{dF}$ & 021930.56 & +000520.3 & & & & \\
\hline $7-21-2001$ & $2 \mathrm{dF}$ & 021931.09 & -004908.4 & & & & \\
\hline $7-21-2001$ & $2 \mathrm{dF}$ & 021941.80 & -004641.5 & & & & \\
\hline $7-21-2001$ & $2 \mathrm{dF}$ & 022017.10 & +000746.3 & & & & \\
\hline $7-21-2001$ & $2 \mathrm{dF}$ & 022251.64 & +002238.0 & & & & \\
\hline $7-21-2001$ & $2 \mathrm{dF}$ & 022308.96 & -002957.0 & & & & \\
\hline $7-21-2001$ & $2 \mathrm{dF}$ & 135213.19 & +015507.5 & & & & \\
\hline $7-21-2001$ & $2 \mathrm{dF}$ & 135320.53 & +022800.4 & & & & \\
\hline $7-21-2001$ & $2 \mathrm{dF}$ & 135344.55 & +02 1734.3 & & & & \\
\hline $7-21-2001$ & $2 \mathrm{dF}$ & 135356.12 & +013423.2 & & & & \\
\hline $7-21-2001$ & $2 \mathrm{dF}$ & 135521.90 & +011604.1 & & & & \\
\hline $7-21-2001$ & $2 \mathrm{dF}$ & 135629.24 & +024039.4 & & & & \\
\hline $7-21-2001$ & $2 \mathrm{dF}$ & 135643.36 & +013258.4 & & & & \\
\hline $7-21-2001$ & $2 \mathrm{dF}$ & 135711.46 & +013105.0 & & & & \\
\hline $7-21-2001$ & $2 \mathrm{dF}$ & 135800.27 & +011423.1 & & & & \\
\hline $7-21-2001$ & $2 \mathrm{dF}$ & 135831.09 & +013345.0 & & & & \\
\hline $7-21-2001$ & $2 \mathrm{dF}$ & 135853.12 & +023529.7 & & & & \\
\hline $7-21-2001$ & $2 \mathrm{dF}$ & 135859.84 & +020509.4 & & & & \\
\hline $7-21-2001$ & $2 \mathrm{dF}$ & 145750.17 & +011502.6 & & & & \\
\hline $7-21-2001$ & $2 \mathrm{dF}$ & 145812.37 & +024456.1 & & & & \\
\hline $7-21-2001$ & $2 \mathrm{dF}$ & 145911.98 & +02 os 09.1 & & & & \\
\hline $7-21-2001$ & $2 \mathrm{dF}$ & 145932.49 & +011104.4 & & & & \\
\hline $7-21-2001$ & $2 \mathrm{dF}$ & 145948.90 & +010446.1 & & & & \\
\hline $7-21-2001$ & $2 \mathrm{dF}$ & 145954.75 & +012413.5 & & & & \\
\hline $7-21-2001$ & $2 \mathrm{dF}$ & 145955.32 & +025722.8 & & & & \\
\hline $7-21-2001$ & $2 \mathrm{dF}$ & 150033.23 & +021710.8 & & & & \\
\hline $7-21-2001$ & $2 \mathrm{dF}$ & 150034.07 & +010106.5 & & & & \\
\hline $7-21-2001$ & $2 \mathrm{dF}$ & 150041.15 & +024133.0 & & & & \\
\hline $7-21-2001$ & $2 \mathrm{dF}$ & 150156.44 & +012846.7 & & & & \\
\hline $7-21-2001$ & $2 \mathrm{dF}$ & 150304.24 & +020331.9 & & & & \\
\hline $7-21-2001$ & $2 \mathrm{dF}$ & 150313.75 & +013346.1 & & & & \\
\hline $7-21-2001$ & $2 \mathrm{dF}$ & 212444.89 & -070251.8 & & & & \\
\hline $7-21-2001$ & $2 \mathrm{dF}$ & 212447.73 & -065608.4 & & & & \\
\hline $7-21-2001$ & $2 \mathrm{dF}$ & 212518.39 & -073544.3 & & & & \\
\hline $7-21-2001$ & $2 \mathrm{dF}$ & 212523.13 & -071723.8 & & & & \\
\hline
\end{tabular}


Table A.1 (cont'd)

\begin{tabular}{|c|c|c|c|c|c|c|c|}
\hline Date & Instrument & $\mathrm{RA}$ & dec & $r$ & $z$ & Type & Comment \\
\hline $7-21-2001$ & $2 \mathrm{dF}$ & $2127 \quad 16.79$ & -072459.7 & & & & \\
\hline $7-21-2001$ & $2 \mathrm{dF}$ & 212729.96 & -073919.0 & & & & \\
\hline $7-21-2001$ & $2 d F$ & 212828.24 & -074115.6 & & & & \\
\hline $7-21-2001$ & $2 \mathrm{dF}$ & 212852.36 & $\begin{array}{llll}-06 & 15 & 01.8\end{array}$ & & & & \\
\hline $7-21-2001$ & $2 \mathrm{dF}$ & 213001.36 & -075456.6 & & & & \\
\hline $7-21-2001$ & $2 \mathrm{dF}$ & 213025.68 & $-07 \quad 3612.9$ & & & & \\
\hline $7-21-2001$ & $2 \mathrm{dF}$ & 213307.37 & -073925.9 & & & & \\
\hline $7-21-2001$ & $2 \mathrm{dF}$ & $2133 \quad 10.90$ & -075131.7 & & & & \\
\hline $7-21-2001$ & $2 \mathrm{dF}$ & 213321.58 & $-07 \quad 1652.3$ & & & & \\
\hline $7-21-2001$ & $2 \mathrm{dF}$ & 213329.97 & -074035.7 & & & & \\
\hline $7-21-2001$ & $2 \mathrm{dF}$ & 213429.54 & -062707.5 & & & & \\
\hline $7-21-2001$ & $2 \mathrm{dF}$ & 213446.91 & $\begin{array}{llll}-07 & 22 & 14.8\end{array}$ & & & & \\
\hline $7-21-2001$ & $2 \mathrm{dF}$ & 213608.57 & -072804.6 & & & & \\
\hline $7-21-2001$ & $2 \mathrm{dF}$ & 213620.35 & $-07 \quad 1235.1$ & & & & \\
\hline $7-21-2001$ & $2 \mathrm{dF}$ & 213623.57 & -081333.6 & & & & \\
\hline $7-21-2001$ & $2 \mathrm{dF}$ & 213657.24 & $\begin{array}{lll}-08 & 05 & 03.7\end{array}$ & & & & \\
\hline $7-21-2001$ & $2 \mathrm{dF}$ & 213713.70 & $\begin{array}{llll}-08 & 07 & 13.2\end{array}$ & & & & \\
\hline $7-21-2001$ & $2 \mathrm{dF}$ & 213802.55 & -073812.6 & & & & \\
\hline $7-21-2001$ & $2 \mathrm{dF}$ & 213807.94 & -071718.1 & & & & \\
\hline $7-21-2001$ & $2 \mathrm{dF}$ & 213824.55 & -073011.7 & & & & \\
\hline $7-21-2001$ & $2 \mathrm{dF}$ & $2141 \quad 16.68$ & -073917.3 & & & & \\
\hline $7-21-2001$ & $2 \mathrm{dF}$ & 214121.75 & -073458.2 & & & & \\
\hline $7-21-2001$ & $2 \mathrm{dF}$ & 214200.01 & -075055.8 & & & & \\
\hline $7-21-2001$ & $2 \mathrm{dF}$ & 214434.01 & -063911.8 & & & & \\
\hline $7-21-2001$ & $2 \mathrm{dF}$ & 214455.37 & -080405.0 & & & & \\
\hline $7-21-2001$ & $2 \mathrm{dF}$ & 214511.79 & -080655.6 & & & & \\
\hline $7-21-2001$ & $2 \mathrm{dF}$ & 214539.65 & -081533.7 & & & & \\
\hline $7-21-2001$ & $2 \mathrm{dF}$ & 214545.30 & -082748.4 & & & & \\
\hline $7-21-2001$ & $2 \mathrm{dF}$ & 214646.85 & $-08 \quad 1519.8$ & & & & \\
\hline $7-21-2001$ & $2 \mathrm{dF}$ & 214708.21 & -075746.7 & & & & \\
\hline $7-21-2001$ & $2 \mathrm{dF}$ & 214734.08 & -074832.7 & & & & \\
\hline $7-21-2001$ & $2 \mathrm{dF}$ & 214736.66 & -075955.4 & & & & \\
\hline $5-10-2002$ & $2 \mathrm{dF}$ & 114257.60 & +001751.6 & & & & \\
\hline $5-10-2002$ & $2 \mathrm{dF}$ & 11439.98 & -000334.0 & & & & \\
\hline $5-10-2002$ & $2 \mathrm{dF}$ & 114419.00 & +001800.9 & & & & \\
\hline $5-10-2002$ & $2 \mathrm{dF}$ & 114524.00 & +005907.1 & & & & \\
\hline $5-10-2002$ & $2 \mathrm{dF}$ & 114630.15 & +002410.3 & & & & \\
\hline $5-10-2002$ & $2 \mathrm{dF}$ & 114720.12 & +004424.4 & & & & \\
\hline
\end{tabular}


Table A.L (cont'd)

\begin{tabular}{|c|c|c|c|c|c|c|c|}
\hline Date & Instrument & RA & dec & $r$ & $z$ & Type & Comment. \\
\hline $5-10-2002$ & $2 \mathrm{dF}$ & 114840.10 & +000834.3 & & & & \\
\hline $5-10-2002$ & $2 \mathrm{dF}$ & 120417.96 & -000753.2 & & & & \\
\hline $5-10-2002$ & $2 \mathrm{dF}$ & 120638.52 & +002311.1 & & & & \\
\hline $5-10-2002$ & $2 \mathrm{~d} F$ & 120828.36 & -005201.6 & & & & \\
\hline $5-10-2002$ & $2 \mathrm{dF}$ & 121044.42 & +001322.9 & & & & \\
\hline $5-10-2002$ & $2 \mathrm{dF}$ & 123714.49 & +000929.4 & & & & \\
\hline $5-10-2002$ & $2 \mathrm{dF}$ & 123718.12 & +000346.2 & & & & \\
\hline $5-10-2002$ & $2 \mathrm{dF}$ & 144258.07 & $\begin{array}{llll}-00 & 12 & 15.7\end{array}$ & & & & \\
\hline $5-10-2002$ & $2 \mathrm{dF}$ & 144320.00 & +00 1926.7 & & & & \\
\hline $5-10-2002$ & $2 \mathrm{dF}$ & 144504.36 & -005324.1 & & & & \\
\hline $5-10-2002$ & $2 \mathrm{dF}$ & 144512.62 & +000703.0 & & & & \\
\hline $5-10-2002$ & $2 \mathrm{dF}$ & 144742.32 & +000726.4 & & & & \\
\hline $5-10-2002$ & $2 \mathrm{dF}$ & 144828.49 & +002014.0 & & & & \\
\hline $5-10-2002$ & $2 \mathrm{dF}$ & 151856.81 & -002138.9 & & & & \\
\hline $5-10-2002$ & $2 \mathrm{dF}$ & 151904.64 & +002845.1 & & & & \\
\hline $5-10-2002$ & $2 \mathrm{dF}$ & 144407.66 & -004334.2 & & 0.21 & Galaxy & Uncertain: near sky lines \\
\hline $5-10-2002$ & $2 \mathrm{dF}$ & 123823.34 & +003019.2 & & 0.1488 & Galaxy & \\
\hline $5-10-2002$ & $2 \mathrm{dF}$ & 151430.58 & $-0008 \quad 03.4$ & & 0.2233 & Galaxy & Emission lines \\
\hline $5-10-2002$ & $2 \mathrm{dF}$ & 123738.14 & +000035.6 & & 0.2276 & Galaxy & \\
\hline $5-10-2002$ & $2 \mathrm{dF}$ & 144917.07 & +002253.0 & & 0.3372 & Galaxy & Emission lines \\
\hline 5-11-2002 & $2 \mathrm{~d} \mathbf{F}$ & 114623.13 & -032630.4 & & & & \\
\hline $5-11-2002$ & $2 \mathrm{dF}$ & 114654.56 & -020402.0 & & & & \\
\hline $5-11-2002$ & $2 \mathrm{dF}$ & 114703.23 & $\begin{array}{llll}-02 & 12 & 47.1\end{array}$ & & & & \\
\hline 5-11-2002 & $2 \mathrm{dF}$ & 114904.74 & -024854.1 & & & & \\
\hline $5-11-2002$ & $2 \mathrm{dF}$ & 141822.51 & +000714.9 & & & & \\
\hline $5-11-2002$ & $2 \mathrm{dF}$ & 141851.63 & +000715.1 & & & & \\
\hline $5-11-2002$ & $2 \mathrm{dF}$ & 141853.36 & -003828.8 & & & & \\
\hline $5-11-2002$ & $2 \mathrm{dF}$ & 141856.16 & +002559.3 & & & & \\
\hline $5-11-2002$ & $2 \mathrm{dF}$ & 141857.35 & $\begin{array}{llll}-00 & 18 & 07.2\end{array}$ & & & & \\
\hline $5-11-2002$ & $2 \mathrm{dF}$ & 141900.17 & +000530.3 & & & & \\
\hline $5-11-2002$ & $2 \mathrm{dF}$ & 141903.55 & +003024.3 & & & & \\
\hline $5-11-2002$ & $2 \mathrm{dF}$ & 141914.68 & -001908.7 & & & & \\
\hline $5-11-2002$ & $2 \mathrm{dF}$ & 141918.60 & $-00 \quad 1259.3$ & & & & \\
\hline $5-11-2002$ & $2 \mathrm{dF}$ & 141959.09 & +001234.2 & & & & \\
\hline $5-11-2002$ & $2 \mathrm{dF}$ & 141959.92 & +000545.2 & & & & \\
\hline $5-11-2002$ & $2 \mathrm{dF}$ & 14. 2032.56 & +00 5317.9 & & & & \\
\hline $5-11-2002$ & $2 \mathrm{dF}$ & 142042.49 & +003624.2 & & & & \\
\hline $5-11-2002$ & $2 \mathrm{dF}$ & 142048.53 & +000648.6 & & & & \\
\hline
\end{tabular}


Table A.1 (cont'd)

\begin{tabular}{|c|c|c|c|c|c|c|c|}
\hline Date & Instrument & $\mathrm{RA}$ & $\operatorname{dec}$ & $r$ & $\mathrm{z}$ & Type & Comment \\
\hline $5-11-2002$ & $2 \mathrm{dF}$ & 142053.52 & -002455.3 & & & & \\
\hline $5-11-2002$ & $2 \mathrm{dF}$ & 142111.42 & .002031 .7 & & & & \\
\hline $5-11-2002$ & $2 \mathrm{dF}$ & 142423.78 & +002433.5 & & & & \\
\hline $5-11-2002$ & $2 \mathrm{dF}$ & 150320.02 & -001920.5 & & & & \\
\hline $5-11-2002$ & $2 \mathrm{dF}$ & 150800.17 & +003406.7 & & & & \\
\hline $5-11-2002$ & $2 \mathrm{dF}$ & 154209.27 & +002817.5 & & & & \\
\hline $5-11-2002$ & $2 \mathrm{dF}$ & 204707.66 & -002413.5 & & & & \\
\hline $5-11-2002$ & $2 \mathrm{dF}$ & 205022.24 & -010734.4 & & & & \\
\hline $5-11-2002$ & $2 \mathrm{dF}$ & 205038.00 & +000850.5 & & & & \\
\hline $5-11-2002$ & $2 \mathrm{dF}$ & 205224.79 & -004943.0 & & & & \\
\hline $5-11-2002$ & $2 \mathrm{dF}$ & 142420.93 & -000753.3 & & & Galaxy & low $\mathrm{z}$ em line galaxy \\
\hline $5-11-2002$ & $2 \mathrm{dF}$ & 204958.56 & -011106.4 & & 0.0 & Star & A star \\
\hline $5-11-2002$ & $2 \mathrm{dF}$ & 141813.56 & +000634.2 & & 0.13 & Galaxy & emission lines \\
\hline $9-14-2002$ & FOCAS & 020655.21 & -005817.4 & & 0.0 & Star & A star eluded psf reject \\
\hline $9-14-2002$ & FOCAS & 031314.03 & -065123.0 & & 0.0226 & Galaxy & Balmer break \\
\hline $9-14-2002$ & FOCAS & 224750.77 & +000149.7 & & 0.0255 & Galaxy & Emission lines \\
\hline $11-8-2002$ & $2 \mathrm{dF}$ & 033149.48 & +002450.3 & & & & Low SB, not detected \\
\hline $11-8-2002$ & $2 \mathrm{dF}$ & 010756.65 & -000106.2 & & & & Low SB: not detected \\
\hline $11-9-2002$ & $2 \mathrm{dF}$ & 005554.29 & +002523.0 & & & & \\
\hline $11-9-2002$ & $2 \mathrm{dF}$ & 005654.83 & +003832.6 & & & & \\
\hline $11-9-2002$ & $2 \mathrm{dF}$ & 010756.65 & -000106.2 & & & & 2ndi obs: tilo overlap \\
\hline $11-9-2002$ & $2 \mathrm{dF}$ & 005509.04 & +003934.2 & & 0.197 & Galaxy & Emission lines \\
\hline $11-9-2002$ & $2 \mathrm{dF}$ & 005951.46 & +005445.7 & & 0.55 & Galaxy & [O II] EM, Balmer abs \\
\hline $10-27-2006$ & DIS III & 164022.00 & +464312.0 & 20.58 & & & \\
\hline $10-27-2006$ & DIS III & 222553.00 & +140004.0 & 18.20 & & & \\
\hline $10-27-2006$ & DIS III & 005338.00 & +004026.0 & 18.24 & & & \\
\hline $10-27-2006$ & DIS III & 233741.00 & +001746.0 & 19.89 & & & \\
\hline $10-27-2006$ & DIS III & 015253.00 & +010253.0 & 19.43 & & & \\
\hline $12-14-2006$ & DIS III & 075050.70 & +205211.3 & 18.13 & 0.063 & Galaxy & EM lines, Weak $\mathrm{H} \beta$ \\
\hline $12-14-2006$ & DIS III & 091753.10 & +514312.0 & 19.34 & 0.21 & Galaxy & At cluster redshift \\
\hline $12-14-2006$ & DIS III & 100051.00 & +232610.0 & 17.72 & 4.58 & Quasar & Image shows ext object \\
\hline $5-12-2007$ & DIS III & 142617.23 & +390145.8 & 20.56 & 0.36 & Galaxy & $\mathrm{EM}$ lines, Weak $\mathrm{H} \beta$ \\
\hline $5-12-2007$ & DIS III & 154718.85 & +205619.5 & 18.68 & 0.264 & Galaxy & Strong Balmer break \\
\hline $5-12-2007$ & DIS III & 164022.00 & +464312.5 & 20.58 & 0.862 & Galaxy & Weak EM lines \\
\hline $6-20-2007$ & DIS III & 155300.05 & +560735.0 & 19.20 & & & Carbon star? \\
\hline $6-20-2007$ & DIS III & 171016.87 & +460302.9 & 20.50 & 0.0835 & Galaxy & Weak EM lines \\
\hline $6-20-2007$ & DIS III & 233723.04 & -003115.6 & 19.74 & 4.53 & Quasar & \\
\hline
\end{tabular}




\section{Bibliography}

Abazajian, K., et al. 2004, AJ, 128, 502

Abell, G. O., Corwin, H. G., \& Olowin, R. P. 1989, ApJS, 70, 1

Adelman-McCarthy, J. K., et al. 2006, ApJS, 162, 38

Adelman-McCarthy, J., et al. 2007, ApJS, preprint doi:10.1086/'518864'

Allam, S. S., et al. 2007, astro-ph/0611138

Alloin, D., Collin-Souffrin, S., \& Joly, M. 1979, ApJS, 37, 361

Angeretti, L., et al. 2005, AJ, 129, 2203

Annibali, F., et al. 2003, AJ, 126, 2752

Asplund, M., et al. 2004, A\&A, 417, 751

Baade, W. 1958, in Galaxies and their Stellar Populations, ed. D. J. K. O'Comell (New York: Interscience), 3

Baker, A. J., et al. 2001, A\&A, 372, L37

Baker, A. J., et al. 2004, ApJ, 604, 125

Baldry, I. K. \& Glazebrook, K. 2003, ApJ, 593, 258

Baldry, I. K., et al. 2004, ApJ, 600, 681

Baldry, I. K., et al. 2005, MNRAS, 358, 441

Baldwin, J. A., Phillips, M. M., \& Terlevich, R. 1981, PASP, 93, 5 
Becker, R. H., White, R. L., \& Helfand, D. J. 1995, ApJ, 450, 559

Beech, M. 1987, Ap\&SS133, 193

Bell, E. F., et al. 2003, ApJS, 149, 289

Bernlöhr, K. 1992, A\&A, 263, 54

Binney, J. \& Tremaine, S. 1987, Galactic Dynamics (Princeton: Princeton University Press)

Binney, J. \& Morrifield, M. 1998, Galactic Astronomy (Princeton: Princeton University Press)

Blanton, M. R., et al. 2003a, AJ, 125, 2348

Blanton, M. R., et al. 2003b, ApJ, 592, 819

Bolzonella, M., Miralles, J.-M. \& Pelló, 2000, A\&A, 363, 476

Bonanos, A. Z., et al. 2004, ApJ, 611, L33

Böhringer, H., et al. 2000, ApJS, 129, 435

Böhringer, H., et al. 2004, A\&A, 425, 367

Boss, A. P. 1988, ApJ, 331, 370

Brinchmann, J., et al. 2004, MNRAS, 351, 1151

Bruzual, G., \& Charlot, S. 2003, MNRAS, 344, 1000

Calzetti, D., Kinney, A. L., \& Storchi-Bergmann, T. 1994, ApJ, 429, 582

Calzetti, D., et al. 2000, ApJ, 533, 682

Cedrés, B., Cepa, J. \& Tomita, A. 2005, ApJ, 634, 1043

Cerviño, M., \&. Luridiana, V. 2005, arXiv:astro-ph/0510411

Chaucer, G. c. 1380, The House of Fame, Book II

Chiu, K. 2006, PhD Thesis

Courteau, S., \& van den Bergh, S. 1999, AJ, 118, 337 
Crone, M. M., et al. 2002, ApJ, 567, 258

Davidson, K., Kinman, T. D., \& Friedman, S. D. 1989, AJ, 97, 1591

De Robertis, M. M., Dufour, R. J., \& Hunt, R. W. 1987, JRASC, 81, 195

Delhaye, J. 1965, in Stars and Stellar Systems, Vol. 5, Galactic Structure, ed. A. Blaaum \& M. Schmidt (Chicago: University of Chicago Press), 61

Dreyer, J. L. E. 1888, Mem. R. Astron. Soc., 49, 1

Dufour, R. J., Garnett, D. R., \& Shields, G. A. 1988, ApJ, 332, 752

Duquennoy, A., \& Mayor, M. 1991, A\&A, 248, 485

Ebeling, H., et al. 1998, MNRAS, 301, 881

Ebeling, H., et al. 2000, MNRAS, 318, 333

Efstathiou, G., Ellis, R. S. \& Peterson, B. A. 1988, MNRAS, 232, 431

Einstein, A. 1936, Science, 84, 506

Elmegreen, B. G. 1997, ApJ, 486, 944

Elmegreen, B. G. 2006, ApJ, 648, 572

Engelbracht, C. W., Rieke, M. J., Rieke, G. H., Kelly, D. M., \& Achtermann, J. M. 1998, ApJ, 505, 639

Esteban, C., et al. 2005, ApJ, 618, L95

Figer, D. F. et al. 2002, ApJ, 581, 258

Figer, D. F. 2005, Nature, 434, 192

Fioc, M., \& Rocca-Volmerange, B. 1997, A\&A, 326, 950

Fischer, D. A., \& Marcy, G. W. 1992, ApJ, 396, 178

Fukugita, M., Ichikawa, T., Gunn, J. E., Doi, M., Shimasaku, K., \& Schncider, D. P. 1996, AJ, 111, 1748 
Gerasimovič, B. P. 1928, Science, 68, 513

Giavalisco, M., Steidel, C. C., \& Szalay, A. S. 1994, ApJ, 425, L5

Giavalisco, M., Steidel, C. C., \& Macchetto, F. D. 1996, ApJ, 470, 189

Grevesse, N., et al. 1984, A\&A, 141, 10

Hall, P. B., et al. 2002, ApJS, 141, 267

Hall, P. B., et al. 2004, AJ, 127, 3146

Hartmann, L. 1998, Accretion Processes in Star Formation (Cambridge: Cambridge University Press)

Hoyle, F. 1953, ApJ, 118, 513

Hunt, L. K., Thuan, T. X., \& Izotov, Y. I. 2003, ApJ, 588, 281

Inada, N., et al. 2005, PASJ, 57, L7

Izotov, Y. I., et al. 1990, Nature, 343, 238

Izotov, Y. I. \& Thuan, T. X. 2004, ApJ, 616, 768

Izotov, Y. I., Thuan, T. X., Guseva, N. G. 2005, ApJ, 632, 210

Izotov, Y. I., et al. 2006, A\&A, 454, 137

Ioka, K., \& Nakamura, T. 2002, ApJ, 570, L21

Jeans, J. H. 1928, Astronomy and Cosmogony (Cambridge: Cambridge University Press)

Jones, C. \& Forman, W. 1999, ApJ, 511, 65

Kashikawa, N., et al. 2002, PASJ, 54, 819

Kauffmann, G., ct al. 2003, MNR $\Lambda$ S, 346, 1055

Kennicutt, R. C. 1983, ApJ, 272, 54

Kennicutt, R. C. 1998a, in ASP Conf. Ser. 142, The Stellar Initial Mass Function, ed., G. Gilmore \& D. Howell (San Francisco: ASP), 1 
Kennicutt, R. C. 1998b, ARA\&A, 36, 189

Kennicutt, R. C., \& Kent, S. M. 1983, AJ, 88, 1094

Kennicutt, R. C., Tamblyn, P., \& Congdon, C. W. 1994, ApJ, 435, 22

Kewley, L. J., \& Dopita, M. A. 2002, ApJS, 142, 35

Kippenhahn, R., \& Weigert, A. 1994, Stellar Structure and Evolution (Berlin: SpringerVerlag:

Kniazev, A. Y., et al. 2003, ApJ, 593, L73

Kniazev, A. Y., et al. 2004, ApJS, 153, 429

Köppen, J., Weidner, C., \& Kroupa, P. 2007, MNRAS, 375, 673

Krolik, J. H. 1999, Active Galactic Nuclei: From the Central Black Hole to the Galactic Environment (Princeton, NJ: Princeton University Press)

Kroupa, P., Tout, C. A., \& Gilmore, G. 1993, MNRAS, 262, 545

Kroupa, P. 2001, MNRAS, 322, 231

Kroupa, P. 2002, Science, 295, 82

Kunth, D. \& Östlin, G. 2000, A\&A Rev., 10, 1

Lambert, D. L. 1978, ApJ, 182, 249

Larson, R. B. 1973, MNRAS, 161, 133

Larson, R. B. 1974, MNRAS, 169, 229

Larson, R. B. 1998, MNRAS, 301, 569

Layzer, D. 1963, ApJ, 137, 351

Le Borgne, D., et al. 2004, A\&A, 425, 881

Lee, H. C., Gibson, B. K., Flynn, C., Kawata, D., \& Beasley, M. A. 2004, MNRAS, 353, 113 
Leitherer, C., Robert, C., \& Heckman, T. M. 1995, ApJS, 99, 173

Leitherer, C. 1998, in ASP Conf. Ser. 142, The Stellar Initial Mass Function, ed., G. Gilmore \& D. Howell (San Francisco: ASP), 61

Lequeux, J., et al. 1979, A\&A, 80, 155

Lewis, I. J., et al. 2002, MNRAS, 333, 270

Low, C., \& Lynden-Bell, D. 1976, MNRAS, 176, 367

Lupton, R. H., Gunn, J. E., \& Szalay, A. S. 1999, AJ, 118, 1406

Lutz, T. E., \& Kelker, D. H. 1973, PASP, 85, 573

Lynn, W. T. 1901, The Observatory, Vol. 24, p. 382

Malkov, O. \& Zinnecker, H. 2001, MNRAS, 321, 149

Mandelbrot, B. B. 1983, The Fractal Geometry of Nature (W. H. Freeman and Co.: New York)

Mayor, M. 1976, A\&A, 48, 301

Mehlert, D., et al. 2001, A\&A, 379, 96

Meier, D. L. 1976a, ApJ, 203, L103

Meier, D. L. 1976b, ApJ, 207, 343

Meléndez, J. 2004, ApJ, 615, 1042

Meyer, M. R. \& Greissl, J. 2005, ApJ, 630, L177

Miller, G. E. \& Scalo, J. M. 1979, ApJS, 41, 513

Nakamura, F., \& Umemura, M. 2002, ApJ, 569, 549

Nakanishi, K, et al. 1997, PASJ, 49, 535

NIST/SEMATECH. 2005, e-Handbook of Statistical Methods, http://www.itl.nist.gov/div898/handbook/ 
Oguri, M., et al. 2004, ApJ, 605, 78

Osterbrock, D. E. 1989, Astrophysics of Gaseous Nebulae and Active Galactic Nuclei (Sausalito, CA: University Science Books)

Padoan, P., Nordlund, Å., \& Jones, B. J. T. 1997, MNRAS, 288, 145

Padoan, P. \& Nordlund, A. 2005, in IMF@50: The Initial Mass Function 50 years later, ed., E. Corbelli, F. Palla \& H. Zinnecker (Kluwer Academic Publishers)

Pagel, B. E. J., et al. 1979, MNRAS, 189, 95

Pei, Y. C. 1992, ApJ, 395, 130

Peimbert, M. \& Costero, R. 1969, Bol. Obs. Tonantzintla y Tacubaya, 5, 3

Pettini, M., et al. 2000, ApJ, 528, 96

Pettini, M., et al. 2002, ApJ, 569, 742

Pettini, M., \& Pagel, B. E. J. 2004, MNRAS, 348, L59

Phelps, R. L., \& Janes, K. A. 1993, AJ, 106, 1870

Pickles, A. J. 1998, PASP, 110, 863P

Press, W. H., Teukolsky, S. A., Vetterling, W. T., \& Flannerty, B. P. 1992, Numerical Recipes in C: The Art of Scientific Computing, Second Edition (Cambridge: Cambridge

Rees, M. J. 1976, MNRAS, 176, 483

Rettura, A., et al. 2006, A\&A, 458, 717

Rieke, G. H., Loken, K, Rieke, M. J., \& Tamblyn, P. 1993, ApJ, 412, 99

Rigby, J. R., \& Rieke, G. H. 2004, ApJ, 606, 237

Robberto, M. et al. 2004, in IMF@50: The Initial Mass Function 50 years later, ed., E. Corbelli, F. Palla \& H. Zinnecker (Kluwer Academic Publishers)

Rolleston, W. R. J., Smartt, S. J., Dufton, P. L. \& Ryans, R. S. I. 2000, A\&A, 363, 537

Sadat, R., et al. 2004, A\&A, 424, 1097 
Salpeter, E. E. 1955, ApJ, 121, 161

Sanner, J. \& Geffert, M. 2001, A\&A, 370, 87

Sargent, W. L. W. \& Searle, L. 1970, ApJ, 162, 155

Sauval, A. J., et al. 1984, ApJ, 282, 330

Scalo, J. M. 1986, Fund. Cosmic Phys., 11, 1

Scalo, J. M. 1998, in ASP Conf. Ser. 142, The Stellar Initial Mass Function, ed., G. Gilmore \& D. Howell (San Francisco: ASP), 201

Scalo, J. 2005, in IMF@50: The Initial Mass Function 50 years later, ed., E. Corbelli, F. Palla \& H. Zinnecker (Kluwer Academic Publishers)

Schlegel, D. J., Finkbeiner, D. P., \& Davis, M. 1998, ApJ, 500, 525

Schmidt, M. 1963, ApJ, 137, 758S

Schneider, D. P., et al. 2003, AJ, 126, 2579

Schombert, J. M., Bothun, G. D., Impey, C. D.,\& Mundy, L. G. 1990, AJ, 1001523

Searle, L. \& Sargent, W. L. W. 1972, ApJ, 173, 25

Seitz, S., et al. 1998, MNRAS, 298, 945

Shapley, A. E., et al. 2003, ApJ, 588, 65

Shaw, R. A. \& Dufour, R. J. 1995, PASP, 107, 896

Sirianni, M., Nota, A., De Marchi, G., Leitherer, C., \& Clampin, M. 2002, ApJ, 579, 275

Skillman, E. D., Kennicutt, R. C., \& Hodge, P. W. 1989, ApJ, 347, 875

Skillman, E. D., et al. 2003, ApJ, 596, 253

Smail, I., et al. 2007, ApJ, 654, L33

Söchting, I. K., et al. 2006, MNRAS, 369, 1334

Spergel, D. N., et al. 2003, ApJS, 148, 175 
Spinrad, H. \& Djorgovski, S. 1984, ApJ, 285, L49

Storchi-Bergmann, T., Calzetti, D., \& Kinney, A. L. 1994, ApJ, 429, 572

Stoughton, C., et al. 2002, AJ, 123, 485

Shadmehri, M. 2004, MNRAS, 354, 375

Stahler, S. W. 1986, PASP, 98, 1081

Steidel, C. C. \& Hamilton, D. 1993, AJ, 105, 2017

Steidel, C. C., et al. 1996a, ApJ, 462, L17

Steidel, C. C., et al. 1996b, AJ, 112, 352

Teplitz, H. I., et al. 2000, ApJ, 533, L5

Thuan, T. X., Izotov, Y. I., \& Lipovetsky, V. A. 1995, ApJ, 445, 108

Thuan, T. X. \& Izotov, Y. I. 2005, ApJS, 161, 240

Tinsley, B. M., \& Gunn, J. E. 1976, ApJ, 203, 52

Tonry, J., \& Davis, M. 1979, AJ, 84, 1511

Tremonti, C. A., et al. 2004, ApJ, 613, 898

Tucker, W. H., Tananbaum, H., \& Remillard, R. A. 1995, ApJ, 444, 532

Tucker, W., et al. 1998, ApJ, 496, L5

Turner, E. L., Ostriker, J. P., \& Gott, J. R. 1984, ApJ, 284, 1

Uomoto, A., et al. 1999, BAAS, 31, 1501

van Zee, L, Salzer, J. J., \& Haynes, M. P. 1998, ApJ, 497, L1

Vikhlinin, A., et al. 1998, ApJ, 502, 558

Vila-Costas, M. B. \& Edmunds, M. G. 1992 MNRAS, 259, 121

Vila-Costas, M. B. \& Edmunds, M. G. 1993 MNRAS, 265, 199 
Walsh, D., Carswell, R. F., \& Weymann, R. J. 1979, Nature, 279, 381

Weidner, C. \& Kroupa, K. 2004, MNRAS, 348, 187

Weidner, C. \& Kroupa, K. 2005, ApJ, 625, 754

Worthey, G. \& Ottaviani, D. L. 1997 ApJS, 111, 377

Wyder, T. K. 2003, AJ, 125, 3097

Yee, H. K. C., et al. 1996a, ApJ, 102, 269

Yee, H. K. C., et al. 1996b, AJ, 111, 1783

Young, P., Gunn, J. E., Oke, J. B., Westphal, J. A., \& Kristian, J. 1980, ApJ, 241, 507

York, D. G., et al. 200, AJ, 120, 1579

Zwicky, F. 1937, Phys. Rev., 51, 290

Zwicky, F. 1965, ApJ, 142, 1293

Zwicky, F. 1966, ApJ, 143, 192 


\section{Vita}

Erik Andrew Hoversten was born on Friday, March 10, 1978 in Iowa City, Iowa. His first home was in Coralville, Iowa but he relocated in 1981 to rural Johnson County a mile outside of Iowa City. He attended pre-school at Our Redeemer Lutheran Church in Iowa City from 1981 to 1983. In 1983 his kindergarten studies took him across town to Lincoln Elementary School where he attended through the sixth grade. From 1990 to 1992 he studied at Northwest Junior High School in Coralville. At Northwest he played football and basketball. In eighth grade he represented the Vikings on the school Mathcounts team placing fifth individually at the regional level and helping the team to a second place in the region and a fifth place finish at the state competition in Des Moines.

In the summer of 1992 he moved with his family to Eagan, Minnesota, a second tier suburb of St. Paul. He attended Eagan Senior High School for four years, graduating in 1996. At Eagan he was a 21 time letter winner earning four letters in cross country, math team, quiz bowl, and knowledge bowl, three letters in track \& field, and two in academics. As a senior he captained the cross country, math, quiz 
bowl and knowledge bowl teams. On the quiz bowl team he became a minor celebrity on Minneapolis Public Access Television. As a sophomore he led the math team in scoring. He left EHS as the second best pole vaulter in school history, with a school record in the pole vault relay.

As a National Merit Finalist he received a four-year, full tuition, room and board scholarship to return to Iowa to attend the Iowa State University of Science and Technology in Ames from 1996 to 2000. At Iowa State he majored in mathematics and physics. He participated in several collegiate mathematics competitions. In addition he played electric guitar in the ISU jazz ensemble for three years and wrote 47 opinion columns for the Iowa State Daily, many of which were picked up by the national college news wire UWIRE. He was inducted into the $\Phi B K$ and IIME honor societies. He received a B.S. in mathematics and a B.S. in physics in 2000, both with distinction. In 1999 he participated in a summer Research Experiences for Undergraduates program at the Smithsonian Astrophysical Observatory working on scattered light images of young stellar objects with Nuria Calvet. At, ISU he searched for planets around white dwarf stars with Steve Kawaler.

In 2000 he moved to Baltimore to join the graduate program in the Department of Physics \& Astronomy at Johns Hopkins University. He did his thesis work under the supervision of Karl Glazebrook. At JHU he was awarded the Roland Prize for Innovation and Excellence in Teaching by the department in 2002. He also played in the JHU Big Band for two years. He spent the first half of 2007 as a visiting student 
at the Centre for Astrophysics and Supercomputing at the Swinburne University of Technology near Melbourne, Australia. He successfully defended his thesis in October, 2007. He is moving to the Pennsylvania State University to talke a post-doctoral appointment with the Swift team. 